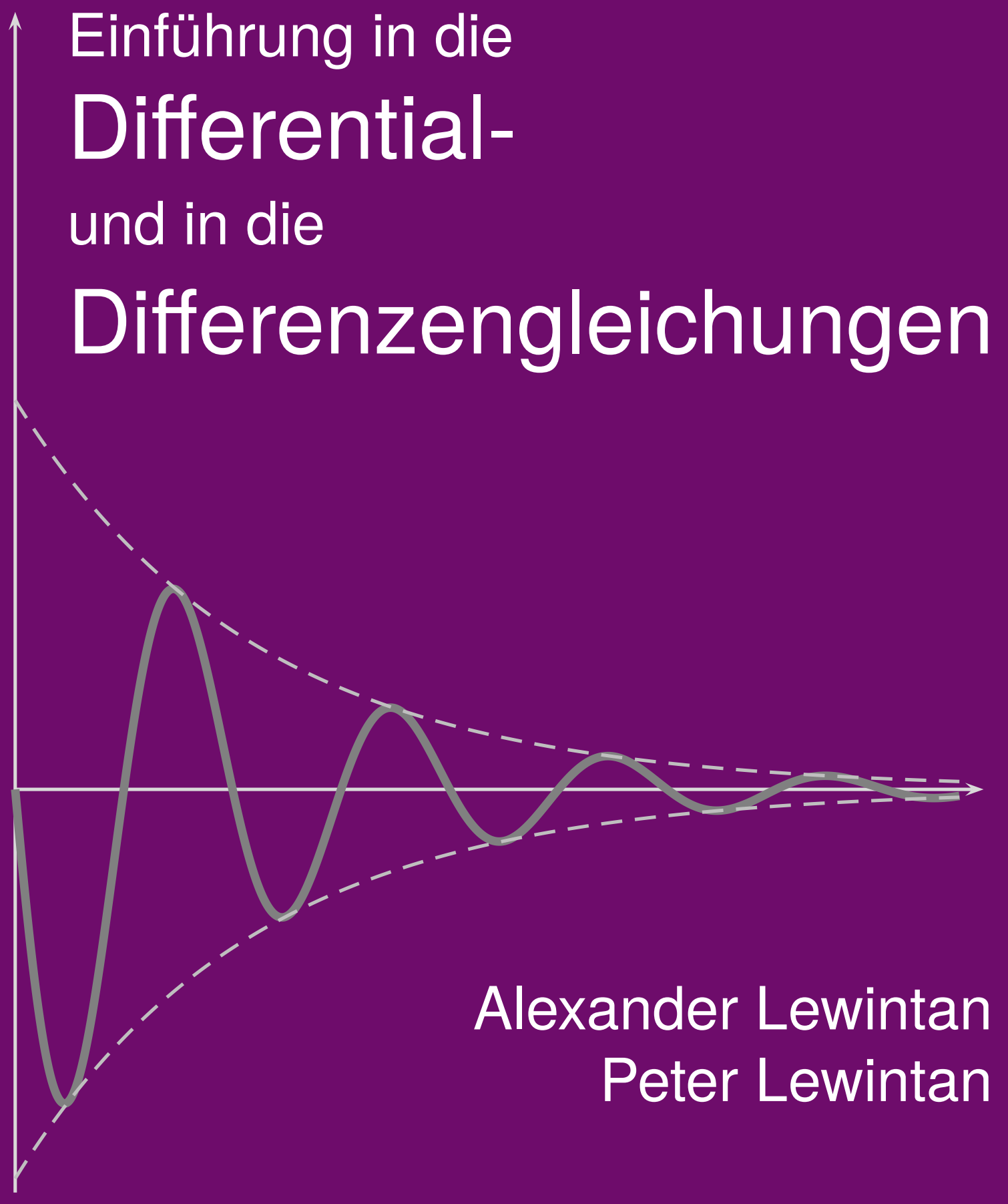

Znoros 



\section{Einführung in die Differential- und in die Differenzengleichungen}

Alexander Lewintan

Peter Lewintan 
Bibliografische Information der Deutschen Nationalbibliothek

Die Deutsche Nationalbibliothek verzeichnet diese Publikation in der Deutschen Nationalbibliografie; detaillierte bibliografische Daten sind im Internet über http://dnb.d-nb.de abrufbar.

\section{(c) (i)}

Das vorliegende Werk ist unter der CC-Lizenz CC-BY 4.0 lizenziert (siehe https://creativecommons.org/licenses/by/4.0/deed.de). Das bedeutet, Sie dürfen das Werk unter Namensnennung teilen (das Material in jedwedem Format oder Medium vervielfältigen und weiterverbreiten) und bearbeiten (das Material remixen, verändern und darauf aufbauen), und zwar für beliebige Zwecke, sogar kommerziell.

Die frei zugängliche Open-Access-Publikation des vorliegenden Titels wurde mit Mitteln des Publikationsfonds der Universitätsbibliothek Duisburg-Essen ermöglicht.

Logos Verlag Berlin GmbH 2022

ISBN 978-3-8325-5448-4

Logos Verlag Berlin GmbH

Georg-Knorr-Str. 4, Geb. 10, 12681 Berlin

Tel.: $\quad+49(0) 30 / 42851090$

Fax: $\quad+49(0) 30 / 42851092$

http://www.logos-verlag.de 


\section{Inhaltsverzeichnis}

1 Lineare Differentialgleichungen erster Ordnung 1

1.1 Anfangswertproblem für lineare Differentialgleichungen erster

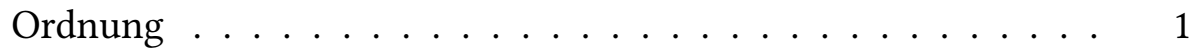

1.2 Lineare homogene Differentialgleichung erster Ordnung . . . . 2

1.3 Lineare inhomogene Differentialgleichung erster Ordnung . . . 6

1.4 Lineare inhomogene Differentialgleichung erster Ordnung mit konstantem Koeffizienten . . . . . . . . . . . . . . . . . . 14

1.5 Auf- und Entladung eines Kondensators . . . . . . . . . . . . . . 24

1.5.1 Aufladung eines Kondensators . . . . . . . . . . . . 24

1.5.2 Entladung eines Kondensators . . . . . . . . . . . . . . 26

1.6 Ein- und Ausschalten eines Stromkreises mit Spule und OHM'schem Widerstand . . . . . . . . . . . . . . . . . . 28

1.6.1 Einschalten des Stroms . . . . . . . . . . . . . . 28

1.6.2 Ausschalten des Stroms . . . . . . . . . . . . 30

2 Lineare Differenzengleichungen erster Ordnung

2.1 Anfangswertproblem für lineare Differenzengleichungen erster Ordnung ......................... 33

2.2 Lineare homogene Differenzengleichung erster Ordnung . . . . 35

2.3 Lineare inhomogene Differenzengleichung erster Ordnung . . . 41

2.4 Lineare inhomogene Differenzengleichung erster Ordnung mit konstantem Koeffizienten . . . . . . . . . . . . . . . 50 
3 Lineare Differentialgleichungen zweiter Ordnung

3.1 Anfangswertproblem für lineare Differentialgleichungen zweiter Ordnung ...................... 65

3.2 Lineare homogene Differentialgleichung zweiter Ordnung . . . 66

3.3 Lineare homogene Differentialgleichung zweiter Ordnung mit konstanten Koeffizienten . . . . . . . . . . . . . . . . 79

3.4 Gedämpfter elektrischer Schwingkreis . . . . . . . . . . . . 85

3.5 Analogien bei mechanischen und elektromagnetischen gedämpften Schwingungen ................... 94

3.6 Lineare inhomogene Differentialgleichung zweiter Ordnung . . 95

3.7 Lineare inhomogene Differentialgleichung zweiter Ordnung mit konstanten Koeffizienten . . . . . . . . . . . . . . . . . . 102

4 Lineare Differenzengleichungen zweiter Ordnung

4.1 Anfangswertproblem für lineare Differenzengleichung zweiter

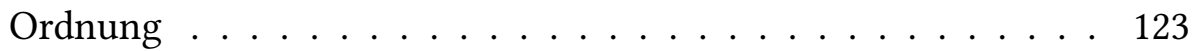

4.2 Lineare homogene Differenzengleichung zweiter Ordnung . . . 126

4.3 Lineare homogene Differenzengleichung zweiter Ordnung mit konstanten Koeffizienten . . . . . . . . . . . . . . . . 137

4.4 Lineare inhomogene Differenzengleichung zweiter Ordnung . 147

4.5 Lineare inhomogene Differenzengleichung zweiter Ordnung mit konstanten Koeffizienten . . . . . . . . . . . . . . . . 156

Schlusswort

Anhang 181

Das Griechische Alphabet . . . . . . . . . . . . . . . . . . . 181

Trigonometrische Formeln . . . . . . . . . . . . . . . . 182

Die einfachsten trigonometrischen Gleichungen . . . . . . . . . . . . 182

Literaturverzeichnis 


\section{Vorwort}

Der Schwerpunkt des vorliegenden Lehrbuches ist es Parallelen bei den Untersuchungen von linearen Differential- und linearen Differenzengleichungen aufzuzeigen. Die Grundlage des Buches bildetete der Vorlesungskurs "Einführung in die Differentialgleichungen und in die Differenzengleichungen” für Informatiker und Wirtschaftsinformatiker der Universität Duisburg-Essen. Für die Digitalisierung der vorliegenden Version danken wir besonders herzlich Larissa Charitonowa! Außerdem gilt unser Dank allen Studierenden, die mit ihren Anmerkungen und Anregungen zur stetigen Verbesserung des Skriptes beigetragen haben. Auch Sie, liebe Leserinnen und Leser, laden wir herzlich ein, uns Ihre Anregungen oder weitere Korrekturvorschläge entgegenzubringen!

Essen, im Januar 2022

Alexander Lewintan und Peter Lewintan 



\section{Lineare Differentialgleichungen erster Ordnung}

Generalvoraussetzung: Im Folgenden bezeichnet $I \subseteq \mathbb{R}$ ein beliebiges Intervall, d.h., $I$ ist eine nichtleere zusammenhängende Teilmenge von $\mathbb{R}$, die ein nichtleeres Inneres hat. Gehört ein Randpunkt zum Intervall, so betrachten wir für die Differenzierbarkeit in diesem Randpunkt einen einseitigen Grenzwert.

\subsection{Anfangswertproblem für lineare Differenti- algleichungen erster Ordnung}

\section{Definition 1.1.1}

Gegeben seien die Funktionen $p, f: I \rightarrow \mathbb{R}$, die auf einem Intervall $I \subseteq \mathbb{R}$ definiert und stetig sind. Gesucht ist eine differenzierbare Funktion $y: I \rightarrow \mathbb{R}$, so dass für alle $x \in I$ gilt:

$$
y^{\prime}(x)+p(x) \cdot y(x)=f(x) .
$$

Gleichung (1.1.1) heißt lineare (gewöhnliche) Differentialgleichung erster Ordnung. Die Funktion $y: I \rightarrow \mathbb{R}$ heißt Lösung der Gleichung (1.1.1) auf dem Intervall I.

Satz und Definition 1.1.2

Seien die Funktionen $p, f: I \rightarrow \mathbb{R}$ auf einem Intervall $I \subseteq \mathbb{R}$ definiert und stetig, sowie $x_{0} \in I$. Dann existiert für beliebiges $y_{0} \in \mathbb{R}$ auf I genau eine Lösung des Anfangswertproblems

$$
\left\{\begin{aligned}
y^{\prime}(x)+p(x) \cdot y(x) & =f(x), \quad x \in I, \\
y\left(x_{0}\right) & =y_{0} .
\end{aligned}\right.
$$


Für einen Beweis verweisen wir auf die spätere Bemerkung 1.3.6.

\section{Bemerkung und Definition 1.1.3}

Ist $f(x)=0$ für alle $x \in I$, so heißt Gleichung (1.1.1) lineare homogene Differentialgleichung erster Ordnung, andernfalls heißt diese lineare inhomogene Differentialgleichung erster Ordnung.

\subsection{Lineare homogene Differentialgleichung ers- ter Ordnung}

Wir untersuchen zuerst die homogene Gleichung

$$
y^{\prime}(x)+p(x) \cdot y(x)=0
$$

(dabei ist die Funktion $p: I \rightarrow \mathbb{R}$ auf $I$ definiert und stetig).

Satz 1.2.1

Sei $y_{1}: I \rightarrow \mathbb{R}$ eine Lösung von (1.1.1h), dann ist $y(x):=c \cdot y_{1}(x)$ ebenfalls eine Lösung von $(1.1 .1 \mathrm{~h})$, wobei $c \in \mathbb{R}$ eine beliebige Konstante ist.

BEwEIS:

Da $y_{1}$ eine Lösung von (1.1.1h) ist, gilt für alle $x \in I$ :

$$
y_{1}^{\prime}(x)+p(x) \cdot y_{1}(x)=0
$$

Dann folgt für $y(x):=c \cdot y_{1}(x)$, wobei $c \in \mathbb{R}$ eine beliebige Konstante ist:

$$
\begin{aligned}
y^{\prime}(x)+p(x) \cdot y(x) & =\left\{c \cdot y_{1}(x)\right\}^{\prime}+p(x) \cdot\left\{c \cdot y_{1}(x)\right\} \\
& =c \cdot y_{1}^{\prime}(x)+p(x) \cdot c \cdot y_{1}(x) \\
& =c \cdot\left(y_{1}^{\prime}(x)+p(x) \cdot y_{1}(x)\right) \\
& \stackrel{(1.2 .1)}{=} 0 \quad \text { für alle } x \in I .
\end{aligned}
$$

Nach Definition 1.1.1 ist also $y(x):=c \cdot y_{1}(x)$ eine Lösung von (1.1.1h) auf $I$, wobei $c \in \mathbb{R}$ eine beliebige Konstante ist. 


\section{Bemerkung 1.2.2}

Da die Funktion $p$ auf I stetig ist, besitzt sie auf I eine Stammfunktion. Sei P : $I \rightarrow \mathbb{R}$ eine Stammfunktion von $p$ auf $I$. Dann ist $y_{1}: I \rightarrow \mathbb{R}$ mit

$$
y_{1}(x):=\mathrm{e}^{-P(x)}
$$

eine Lösung von (1.1.1h) auf I. In der Tat:

$$
\begin{aligned}
y_{1}^{\prime}(x) & =(-P(x))^{\prime} \cdot \mathrm{e}^{-P(x)}=-p(x) \cdot \mathrm{e}^{-P(x)} \quad \text { und } \\
y_{1}^{\prime}(x)+p(x) \cdot y_{1}(x) & =-p(x) \cdot \mathrm{e}^{-P(x)}+p(x) \cdot \mathrm{e}^{-P(x)}=0 \quad \text { auf } \quad I .
\end{aligned}
$$

\section{Satz und Definition 1.2.3}

Allgemeine Lösung der linearen homogenen Differentialgleichung erster Ordnung

fede Lösung $y: I \rightarrow \mathbb{R}$ von (1.1.1h) lässt sich in der Form $y(x):=c \cdot y_{1}(x)$ darstellen, wobei

$$
y_{1}(x)=\mathrm{e}^{-P(x)},
$$

$P: I \rightarrow \mathbb{R}$ eine Stammfunktion von $p$ und $c \in \mathbb{R}$ eine Konstante ist.

Lässt man in $c \cdot y_{1}(x)$ die Zahl c die Menge der reellen Zahlen durchlaufen, so erhält man alle Lösungen der Gleichung (1.1.1h). Man sagt: Durch $y(x)=c \cdot y_{1}(x)$ ist die allgemeine Lösung der linearen homogenen Differentialgleichung erster Ordnung (1.2.1) gegeben.

BEWEIS:

Nach Bemerkung 1.2.2 und Satz 1.2.1 ist jede Funktion $y: I \rightarrow \mathbb{R}$ mit

$$
y(x):=c \cdot y_{1}(x) \quad \text { und } \quad y_{1}(x)=\mathrm{e}^{-P(x)}
$$

immer eine Lösung von (1.1.1h) für alle $c \in \mathbb{R}$.

Sei umgekehrt $\widetilde{y}: I \rightarrow \mathbb{R}$ eine beliebige Lösung von (1.1.1h) und $y_{1}$ (durch eine beliebige aber feste Wahl der Stammfunktion $P$ ) fest gewählt. Wir zeigen: Es existiert ein $\widetilde{c} \in \mathbb{R}$, sodass auf dem ganzen Intervall $I$ gilt

$$
\widetilde{y}(x)=\widetilde{c} \cdot y_{1}(x) .
$$

Da $\widetilde{y}$ eine Lösung von (1.1.1h) ist, folgt

$$
\widetilde{y}^{\prime}(x)+p(x) \cdot \widetilde{y}(x)=0 \quad \text { für alle } x \in I .
$$


Die Funktion $z: I \rightarrow \mathbb{R}$ mit

$$
z(x):=\frac{\widetilde{y}(x)}{y_{1}(x)}
$$

ist auf ganz $I$ definiert (es gilt stets $y_{1}(x)>0$ ), differenzierbar und es folgt

$$
\begin{aligned}
z^{\prime}(x) & =\frac{\widetilde{y}^{\prime}(x) \cdot y_{1}(x)-\widetilde{y}(x) \cdot y_{1}^{\prime}(x)}{\left(y_{1}(x)\right)^{2}} \\
& =\frac{-p(x) \cdot \widetilde{y}(x) \cdot y_{1}(x)-\widetilde{y}(x) \cdot\left(-p(x) \cdot y_{1}(x)\right)}{\left(y_{1}(x)\right)^{2}} \\
& =0 \quad \text { für alle } x \in I .
\end{aligned}
$$

Dann ist $z$ konstant auf dem ganzen Intervall $I$. Also existiert ein $\widetilde{c} \in \mathbb{R}$ mit

$$
\frac{\widetilde{y}(x)}{y_{1}(x)}=\widetilde{c} \text { für alle } x \in I .
$$

Somit ist jede Lösung von (1.1.1h) in der Form $c \cdot y_{1}(x)$ mit $y_{1}(x)=\mathrm{e}^{-P(x)}$ und geeignetem $c \in \mathbb{R}$ darstellbar.

\section{Beispiel 1.2.4}

$$
y^{\prime}(x)-\tan (x) \cdot y(x)=0, \quad x \in\left(-\frac{\pi}{2} ; \frac{\pi}{2}\right)
$$

Es gilt für jedes $x \in\left(-\frac{\pi}{2} ; \frac{\pi}{2}\right): \quad p(x)=-\tan (x)$, und da $\cos (x)>0$ auf $\left(-\frac{\pi}{2} ; \frac{\pi}{2}\right)$ folgt:

$$
\int p(x) \mathrm{d} x=-\int \tan (x) \mathrm{d} x=\ln (\cos (x))+m, \quad m \in \mathbb{R} .
$$

Wir nehmen (z.B.) $m=0$, dann ist

$$
P(x)=\ln (\cos (x))
$$

und

$$
y_{1}(x)=\mathrm{e}^{-P(x)}=\frac{1}{\cos (x)} .
$$

Die allgemeine Lösung der Gleichung (1.2.3) lautet somit nach Satz 1.2.3:

$$
y(x)=\frac{c}{\cos (x)} \quad \text { mit beliebigem } c \in \mathbb{R} .
$$




\section{Folgerung 1.2.5 (Eindeutigkeit der Lösung des Anfangswertproblems)}

Sei die Funktion $p: I \rightarrow \mathbb{R}$ auf I definiert und stetig, sowie $x_{0} \in I$. Dann existiert für beliebiges $y_{0} \in \mathbb{R}$ auf I genau eine Lösung des Anfangswertproblems

$$
\left\{\begin{aligned}
y^{\prime}(x)+p(x) \cdot y(x) & =0, \\
y\left(x_{0}\right) & =y_{0} .
\end{aligned}\right.
$$

BEWEIS:

Da die Funktion $p$ auf $I$ stetig ist, ist die Funktion $P: I \rightarrow \mathbb{R}$ mit

$$
P(x):=\int_{x_{0}}^{x} p(t) \mathrm{d} t
$$

eine Stammfunktion von $p$ auf $I$. Nach Satz 1.2.3 ist die Funktion $y: I \rightarrow \mathbb{R}$ mit

$$
y(x):=c \cdot \mathrm{e}^{-\int_{x_{0}}^{x} p(t) \mathrm{d} t}
$$

und beliebigem $c \in \mathbb{R}$ die allgemeine Lösung der Gleichung

$$
y^{\prime}(x)+p(x) \cdot y(x)=0 \quad \text { auf } \quad I .
$$

Mit dem Anfangswert $y_{0} \in \mathbb{R}$ können wir die Konstante bereits eindeutig anpassen:

$$
y\left(x_{0}\right)=y_{0} \quad \Leftrightarrow \quad c \cdot \mathrm{e}^{-\int_{x_{0}}^{x_{0}} p(t) \mathrm{d} t}=y_{0} \quad \Leftrightarrow \quad c=y_{0} .
$$

Also ist die Funktion $y: I \rightarrow \mathbb{R}$ mit

$$
y(x)=y_{0} \cdot \mathrm{e}^{-\int_{x_{0}}^{x} p(t) \mathrm{d} t}
$$

die eindeutig bestimmte Lösung des Anfangswertproblems (1.2.4).

\section{Beispiel 1.2.6 (Gesetz des radioaktiven Zerfalls)}

Sei $N:=N(t)$ die Zahl der zur Zeit $t \in \mathbb{R}$ noch nicht zerfallenen Kerne eines radioaktiven Stoffes. Die Anzahl der Kerne, die radioaktiv zerfallen können, nimmt mit der Zeit ab und es gilt:

$$
\dot{N}(t)=-\lambda \cdot N(t)
$$


mit einer Konstante $\lambda \in \mathbb{R}^{>0}$. Mit anderen Worten gilt

$$
\dot{N}(t)+\lambda \cdot N(t)=0, \quad \text { für alle } t \in \mathbb{R} .
$$

Sei $t_{0}=0$ der Anfangspunkt der Prozessbeobachtung. Mit der Anfangsbedingung $N(0)=N_{0}$ erhalten wir nach Folgerung 1.2.5 durch

$$
N(t)=N_{0} \cdot \mathrm{e}^{-\int_{0}^{t} \lambda \mathrm{d} \tau}=N_{0} \cdot \mathrm{e}^{-\lambda t}
$$

die eindeutig bestimmte Lösung dieses Anfangswertproblems.

Beachte: Für $t>t_{0}$ bzw. $t<t_{0}$ zeigt die Lösung die Anzahl der nicht zerfallenen Kerne nach bzw. vor dem Start der Beobachtung.

\section{Bemerkung 1.2.7}

Lineare homogene Differentialgleichungen erster Ordnung mit konstantem Koeffizienten

Sei $p \in \mathbb{R}$. Gesucht ist eine Funktion $y: \mathbb{R} \rightarrow \mathbb{R}$, sodass für alle $x \in \mathbb{R}$ gilt:

$$
y^{\prime}(x)+p \cdot y(x)=0 .
$$

Offensichtlich ist die Funktion $P: \mathbb{R} \rightarrow \mathbb{R}$ mit $P(x):=p \cdot x$ eine Stammfunktion der konstanten Funktion $p: \mathbb{R} \rightarrow \mathbb{R}$ mit $p(x):=p$ auf $\mathbb{R}$. Nach Bemerkung 1.2.2 ist die Funktion $y_{1}: \mathbb{R} \rightarrow \mathbb{R}$ mit $y_{1}(x):=\mathrm{e}^{-p \cdot x}$ eine Lösung und nach Satz 1.2.3 ist

$$
y(x)=c \cdot y_{1}(x)=c \cdot \mathrm{e}^{-p \cdot x}
$$

mit beliebigem $c \in \mathbb{R}$ die allgemeine Lösung der Gleichung (1.2.6) auf $\mathbb{R}$.

\subsection{Lineare inhomogene Differentialgleichung erster Ordnung}

\section{Satz 1.3.1}

Seien die Funktionen $p, f: I \rightarrow \mathbb{R}$ auf einem Intervall I definiert und stetig. Dann lässt sich die Lösungsmenge $\mathbb{L}$ der inhomogenen Differentialgleichung erster Ordnung

$$
y^{\prime}(x)+p(x) \cdot y(x)=f(x)
$$


auf I schreiben als: $\mathbb{L}=y^{*}(x)+\mathbb{L}_{0}$, wobei $\mathbb{L}_{0}$ die Lösungsmenge der entsprechenden homogenen Differentialgleichung

$$
y^{\prime}(x)+p(x) \cdot y(x)=0
$$

und $y^{*}(x)$ eine (sogenannte partikuläre) Lösung der inhomogenen Differentialgleichung ist.

BEweIs:

Wir haben bereits in Satz 1.2.3 gesehen, dass $\mathbb{L}_{0}$ nichtleer ist. Wir werden in Bemerkung 1.3.4 zeigen, dass auch $\mathbb{L} \neq \varnothing$, mit anderen Worten existiert eine partikuläre Lösung $y^{*}(x)$ von (1.3.1) und es gilt auf $I$

$$
\left(y^{*}\right)^{\prime}(x)+p(x) \cdot y^{*}(x)=f(x) .
$$

1. Sei $y_{L}(x) \in \mathbb{L}$ eine beliebige Lösung von (1.3.1). Dann gilt

$$
y_{L}^{\prime}(x)+p(x) \cdot y_{L}(x)=f(x)
$$

auf $I$ und somit

$$
\begin{aligned}
y_{L}^{\prime}(x)-\left(y^{*}\right)^{\prime}(x)+p(x) \cdot\left(y_{L}(x)-y^{*}(x)\right) & =0 \\
\Leftrightarrow \quad & \left(y_{L}(x)-y^{*}(x)\right)^{\prime}+p(x) \cdot\left(y_{L}(x)-y^{*}(x)\right)=0 .
\end{aligned}
$$

D.h., $\widetilde{y}(x):=y_{L}(x)-y^{*}(x)$ ist eine Lösung der homogenen Differentialgleichung

$$
y^{\prime}(x)+p(x) \cdot y(x)=0
$$

auf $I$. Also gilt für jedes $y_{L}(x) \in \mathbb{L}$ :

$$
y_{L}(x)=y^{*}(x)+\widetilde{y}(x) \in y^{*}(x)+\mathbb{L}_{0}
$$

und

$$
\mathbb{L} \subseteq y^{*}(x)+\mathbb{L}_{0}
$$

2. Sei nun $\widetilde{y}(x) \in \mathbb{L}_{0}$ eine beliebige Lösung der homogenen Differentialgleichung. Dann gilt auf $I$ :

$$
\widetilde{y}^{\prime}(x)+p(x) \cdot \widetilde{y}(x)=0
$$


Da $y^{*}(x)$ eine partikuläre Lösung der inhomogenen Differentialgleichung (1.3.1) ist, folgt

$$
\begin{aligned}
\widetilde{y}^{\prime}(x)+\left(y^{*}\right)^{\prime}(x)+p(x) \cdot\left(\widetilde{y}(x)+y^{*}(x)\right) & =f(x) \\
\Leftrightarrow \quad\left(\widetilde{y}(x)+y^{*}(x)\right)^{\prime}+p(x) \cdot\left(\widetilde{y}(x)+y^{*}(x)\right) & =f(x) .
\end{aligned}
$$

D.h., $y(x):=\widetilde{y}(x)+y^{*}(x)$ ist eine Lösung der inhomogenen Differentialgleichung (1.3.1) auf $I$. Also gilt für jedes $\widetilde{y}(x) \in \mathbb{L}_{0}$ :

$$
y(x):=y^{*}(x)+\widetilde{y}(x) \in \mathbb{L}
$$

und

$$
y^{*}(x)+\mathbb{L}_{0} \subseteq \mathbb{L}
$$

Insgesamt haben wir $\mathbb{L}=y^{*}(x)+\mathbb{L}_{0}$ gezeigt.

\section{Folgerung 1.3.2}

Wie wir schon wissen, ist

$$
y_{1}(x)=\mathrm{e}^{-P(x)}
$$

immer eine Lösung der homogenen Differentialgleichung (1.1.1h) auf I. (Dabei ist $P: I \rightarrow \mathbb{R}$ eine Stammfunktion von $p$ auf I.) Lässt man in $c \cdot y_{1}(x)$ die Zahl $c$ die Menge der reellen Zahlen durchlaufen, so erhält man alle Lösungen der Gleichung (1.1.1h).

Also gilt für die Lösungsmenge $\mathbb{L}_{0}$ :

$$
\mathbb{L}_{0}=\left\{c \cdot y_{1}(x): c \in \mathbb{R}\right\},
$$

dabei heißt c $\cdot y_{1}(x)$ die allgemeine Lösung der homogenen Differentialgleichung. Ist jetzt $y^{*}(x)$ eine partikuläre Lösung der inhomogenen Differentialgleichung (1.3.1), dann gilt nach Satz 1.3.1 für die Lösungsmenge $\mathbb{L}$ :

$$
\mathbb{L}=\left\{c \cdot y_{1}(x)+y^{*}(x): c \in \mathbb{R}\right\} .
$$

Man sagt: Durch

$$
y(x)=c \cdot y_{1}(x)+y^{*}(x)
$$

ist die allgemeine Lösung der linearen inhomogenen Differentialgleichung erster Ordnung (1.3.1) gegeben. 
Oder auch: Die allgemeine Lösung der linearen inhomogenen Differentialgleichung erster Ordnung hat die Form

$$
y(x)=\widetilde{y}(x)+y^{*}(x),
$$

wobei $\widetilde{y}(x)$ die allgemeine Lösung der entsprechenden linearen homogenen Differentialgleichung erster Ordnung und $y^{*}(x)$ eine partikuläre Lösung der inhomogenen Differentialgleichung ist.

\section{Beispiel 1.3.3}

$$
y^{\prime}(x)-3 y(x)=x, \quad x \in \mathbb{R} .
$$

1) Die entsprechende homogene Differentialgleichung lautet

$$
y^{\prime}(x)-3 y(x)=0 .
$$

Hier gilt $p=-3$ und nach Bemerkung 1.2.7 ist

$$
\widetilde{y}(x)=c \cdot \mathrm{e}^{3 x}
$$

mit beliebigem $c \in \mathbb{R}$ die allgemeine Lösung der entsprechenden homogenen Differentialgleichung (1.3.3h) auf $\mathbb{R}$.

2) Wie ist nun $y^{*}(x)$ zu wählen?

Dazu betrachten wir den Ansatz

$$
y^{*}(x):=A \cdot x+B
$$

mit $A, B \in \mathbb{R}$. Dann gilt für jedes $x \in \mathbb{R}$ :

$$
\begin{aligned}
\left(y^{*}\right)^{\prime}(x) & =A \quad \text { und } \quad\left(y^{*}\right)^{\prime}(x)-3 y^{*}(x)=x \\
& \Leftrightarrow \quad A-3 \cdot(A \cdot x+B)=x \\
& \Leftrightarrow \quad-3 A \cdot x+(A-3 B)=x
\end{aligned}
$$

Ein Koeffizientenvergleich liefert:

$$
\left\{\begin{array} { l } 
{ - 3 A = 1 } \\
{ A - 3 B = 0 }
\end{array} \Leftrightarrow \left\{\begin{array}{l}
A=-\frac{1}{3} \\
B=-\frac{1}{9}
\end{array}\right.\right.
$$


Dann ist

$$
y^{*}(x)=-\frac{1}{3} \cdot x-\frac{1}{9}
$$

eine partikuläre Lösung der inhomogenen Gleichung. Nach Satz 1.3.1 und Folgerung 1.3.2 ist

$$
y(x)=\widetilde{y}(x)+y^{*}(x)=c \cdot \mathrm{e}^{3 x}+\left(-\frac{1}{3} \cdot x-\frac{1}{9}\right)
$$

mit $c \in \mathbb{R}$ die allgemeine Lösung der inhomogenen Gleichung (1.3.3) auf $\mathbb{R}$.

\section{Bemerkung 1.3.4 (Variation der Konstanten)}

Seien die Funktionen $p, f: I \rightarrow \mathbb{R}$ auf einem Intervall I definiert und stetig. Sei $P: I \rightarrow \mathbb{R}$ eine Stammfunktion von $p$ auf $I$. Nach Bemerkung 1.2.2 ist $y_{1}: I \rightarrow \mathbb{R}$ mit

$$
y_{1}(x):=\mathrm{e}^{-P(x)}
$$

eine Lösung der homogenen Differentialgleichung

$$
y^{\prime}(x)+p(x) \cdot y(x)=0
$$

auf I. Eine partikuläre Lösung der inhomogenen Differentialgleichung

$$
y^{\prime}(x)+p(x) \cdot y(x)=f(x)
$$

findet man nach dem Verfahren der Variation der Konstanten:

Wir suchen eine partikuläre Lösung in der Form

$$
y^{*}(x)=c(x) \cdot y_{1}(x),
$$

wobei c $: I \rightarrow \mathbb{R}$ eine unbekannte Funktion ist. Dann gilt (mit Voraussetzung, dass die Funktion $c$ in I differenzierbar ist):

$$
\left(y^{*}\right)^{\prime}(x)=c^{\prime}(x) \cdot y_{1}(x)+c(x) \cdot y_{1}^{\prime}(x) .
$$

In die Differentialgleichung (1.3.1) eingesetzt ergibt das:

$$
\begin{aligned}
& & \left(c^{\prime}(x) \cdot y_{1}(x)+c(x) \cdot y_{1}^{\prime}(x)\right)+p(x) \cdot c(x) \cdot y_{1}(x) & =f(x) \\
& \Leftrightarrow & c^{\prime}(x) \cdot y_{1}(x)+c(x) \cdot \underbrace{\left(y_{1}^{\prime}(x)+p(x) \cdot y_{1}(x)\right)}_{=0} & =f(x) \\
& \Leftrightarrow & c^{\prime}(x) \cdot y_{1}(x) & =f(x),
\end{aligned}
$$

wobei wir ausgenutzt haben, dass die Funktion $y_{1}$ eine Lösung der homogenen Differentialgleichung ist. 
Da $y_{1}(x)=\mathrm{e}^{-P(x)} \neq 0$ für alle $x \in I$ ist, ist die Gleichung $c^{\prime}(x) \cdot y_{1}(x)=f(x)$ eindeutig lösbar. Es gilt somit

$$
c^{\prime}(x)=\mathrm{e}^{P(x)} \cdot f(x) .
$$

Da die Funktion $\mathrm{e}^{P(x)} \cdot f(x)$ auf I stetig ist, besitzt sie dort eine Stammfunktion.

\section{Beispiel 1.3.5}

$$
y^{\prime}(x)-\cot (x) \cdot y(x)=\sin (x), \quad x \in(0 ; \pi) .
$$

1) Die entsprechende homogene Differentialgleichung lautet

$$
y^{\prime}(x)-\cot (x) \cdot y(x)=0 .
$$

Es gilt für alle $x \in(0 ; \pi)$ mit $p(x):=-\cot (x)$, und da $\sin (x)>0$ auf $(0 ; \pi)$ folgt

$$
\int p(x) \mathrm{d} t=-\int \cot (x) \mathrm{d} t=-\ln (\sin (x))+m, \quad m \in \mathbb{R} .
$$

Wir nehmen (z.B.) $m=0$, dann ist $\quad P(x)=-\ln (\sin (x)) \quad$ auf $(0 ; \pi)$. Nach Bemerkung 1.2.7 ist

$$
\widetilde{y}(x)=\widetilde{c} \cdot \sin (x)
$$

mit beliebigem $\widetilde{c} \in \mathbb{R}$ die allgemeine Lösung der entsprechenden homogenen (1.3.5h) Differentialgleichung.

2) Wir suchen nun $y^{*}(x)$ mit dem Verfahren der Variation der Konstanten. Nach Bemerkung 1.3.4 erhalten wir auf dem offenen Intervall $(0 ; \pi)$ :

$$
c^{\prime}(x)=\mathrm{e}^{-\ln (\sin (x))} \cdot \sin (x)=\frac{1}{\sin (x)} \cdot \sin (x)=1 .
$$

Somit ist $c(x)=x+n$, mit $n \in \mathbb{R}$. Mit $n=0$ bekommen wir folgende partikuläre Lösung

$$
y^{*}(x)=c(x) \cdot y_{1}(x)=x \cdot \sin (x) .
$$

Die allgemeine Lösung der inhomogenen Gleichung $(1.3 .5)$ auf $(0 ; \pi)$ ist somit

$$
y(x)=\widetilde{y}(x)+y^{*}(x)=\widetilde{c} \cdot \sin (x)+x \cdot \sin (x) \quad \text { mit } \quad \widetilde{c} \in \mathbb{R} .
$$


Beachte: Diese Funktion ist natürlich auch außerhalb des Intervalls $(0 ; \pi)$ wohldefiniert, die Einschränkung des Intervalls kommt aber daher, dass wir ein Intervall benötigen auf dem der Kotangens aus der Differentialgleichung (1.3.5) stetig ist.

\section{Bemerkung 1.3.6}

Eindeutigkeit der Lösung des Anfangswertproblems. (Beweis des Satzes 1.1.2)

$$
\left\{\begin{aligned}
y^{\prime}(x)+p(x) \cdot y(x) & =f(x), \quad x \in I, \\
y\left(x_{0}\right) & =y_{0} .
\end{aligned}\right.
$$

BeweIs:

Da die Funktion $p$ auf $I$ stetig ist, ist die Funktion $P: I \rightarrow \mathbb{R}$ mit

$$
P(x)=\int_{x_{0}}^{x} p(t) \mathrm{d} t
$$

eine Stammfunktion von $p$ auf $I$. Nach Satz 1.2.3 ist die Funktion $\widetilde{y}: I \rightarrow \mathbb{R}$ mit

$$
\widetilde{y}(x):=\widetilde{c} \cdot \mathrm{e}^{-P(x)}
$$

und mit beliebigem $\widetilde{c} \in \mathbb{R}$ die allgemeine Lösung der entsprechenden homogenen Differentialgleichung

$$
y^{\prime}(x)+p(x) \cdot y(x)=0
$$

auf $I$. Die Funktion $c: I \rightarrow \mathbb{R}$ mit

$$
c(x):=\int_{x_{0}}^{x} \mathrm{e}^{P(t)} \cdot f(t) \mathrm{d} t
$$

ist eine Stammfunktion von $\mathrm{e}^{P(x)} \cdot f(x)$ auf $I$. Nach Bemerkung 1.3.4 ist durch die Funktion $y^{*}: I \rightarrow \mathbb{R}$ mit

$$
y^{*}(x):=c(x) \cdot \mathrm{e}^{-P(x)}
$$

eine partikuläre Lösung und durch

$$
y(x)=\widetilde{y}(x)+y^{*}(x)=(\widetilde{c}+c(x)) \cdot \mathrm{e}^{-P(x)}
$$

die allgemeine Lösung der inhomogenen Gleichung

$$
y^{\prime}(x)+p(x) \cdot y(x)=f(x)
$$

auf $I$ gegeben. 
Mit dem Anfangswert $y_{0} \in \mathbb{R}$ können wir nun die Konstante $\widetilde{c}$ bereits eindeutig anpassen:

$$
\begin{array}{rlrlrl}
y\left(x_{0}\right) & =y_{0} & \Leftrightarrow & \left(\widetilde{c}+c\left(x_{0}\right)\right) \cdot \mathrm{e}^{-P\left(x_{0}\right)}=y_{0} \\
\Leftrightarrow & \widetilde{c} & =y_{0}, \quad \text { denn } & c\left(x_{0}\right)=0 \quad \text { und } & \mathrm{e}^{-P\left(x_{0}\right)}=1 .
\end{array}
$$

Also ist die Funktion $y: I \rightarrow \mathbb{R}$ mit

$$
y(x):=\left(y_{0}+c(x)\right) \cdot \mathrm{e}^{-P(x)}
$$

die eindeutig bestimmte Lösung des Anfangswertproblems (1.3.6).

\section{Satz 1.3.7 (Superpositionsprinzip)}

Seien die Funktionen $p, f_{1}, f_{2}: I \rightarrow \mathbb{R}$ auf einem Intervall I definiert und stetig. Seien weiter $y_{1}^{*}(x)$ eine partikuläre Lösung von

$$
y^{\prime}(x)+p(x) \cdot y(x)=f_{1}(x)
$$

und $y_{2}^{*}(x)$ eine partikuläre Lösung von

$$
y^{\prime}(x)+p(x) \cdot y(x)=f_{2}(x) .
$$

Dann ist

$$
y^{*}(x):=y_{1}^{*}(x)+y_{2}^{*}(x)
$$

eine partikuläre Lösung von

$$
y^{\prime}(x)+p(x) \cdot y(x)=f_{1}(x)+f_{2}(x) .
$$

BEWEIS:

Einsetzen von $y^{*}(x)$ in (1.3.8) liefert die Behauptung, weil $y_{1}^{*}(x)$ und $y_{2}^{*}(x)$ partikuläre Lösungen der entsprechenden Gleichungen sind:

In der Tat gilt für jedes $x \in I$ :

$$
\left(y^{*}\right)^{\prime}(x)=\left(y_{1}^{*}\right)^{\prime}(x)+\left(y_{2}^{*}\right)^{\prime}(x),
$$

sodass

$$
\begin{aligned}
& \left(y^{*}\right)^{\prime}(x)+p(x) \cdot y^{*}(x)=\left(y_{1}^{*}\right)^{\prime}(x)+\left(y_{2}^{*}\right)^{\prime}(x)+p(x) \cdot\left(y_{1}^{*}(x)+y_{2}^{*}(x)\right) \\
& =\left[\left(y_{1}^{*}\right)^{\prime}(x)+p(x) \cdot y_{1}^{*}(x)\right]+\left[\left(y_{2}^{*}\right)^{\prime}(x)+p(x) \cdot y_{2}^{*}(x)\right] \\
& \underset{(1.3 .7 \mathrm{~b})}{\stackrel{(1.3 .7 \mathrm{a})}{=}} f_{1}(x)+f_{2}(x) \text {. }
\end{aligned}
$$




\subsection{Lineare inhomogene Differentialgleichung erster Ordnung mit konstantem Koeffizien- ten}

\section{Bemerkung 1.4.1}

Gegeben seien eine Funktion $f: I \rightarrow \mathbb{R}$, die auf einem Intervall I definiert und stetig ist, und eine reelle Zahl $p \in \mathbb{R}$. Gesucht ist eine Funktion $y: I \rightarrow \mathbb{R}$, sodass für alle $x \in I$ gilt:

$$
y^{\prime}(x)+p \cdot y(x)=f(x) .
$$

Nach Folgerung 1.3.2 und Bemerkung 1.2.7 hat die allgemeine Lösung der linearen inhomogenen Differentialgleichung (1.4.1) die Form $y(x)=\widetilde{y}(x)+y^{*}(x)$, wobei

$$
\widetilde{y}(x)=c \cdot \mathrm{e}^{-p \cdot x}
$$

mit beliebigem $c \in \mathbb{R}$, die allgemeine Lösung der entsprechenden linearen homogenen Differentialgleichung

$$
y^{\prime}(x)+p \cdot y(x)=0
$$

und $y^{*}(x)$ eine partikuläre Lösung der inhomogenen Differentialgleichung ist. In Bemerkung 1.3.4 haben wir $y^{*}(x)$ mit dem Verfahren der Variation der Konstanten gesucht. Wir betrachten nun die Form partikulärer Lösungen $y^{*}(x)$ von (1.4.1) für einige Sonderfälle der rechten Seite $f: \mathbb{R} \rightarrow \mathbb{R}$.

i) $f(x):=\mathrm{e}^{\gamma \cdot x} \cdot P_{n}(x)$, wobei $\gamma \in \mathbb{R}$ und $P_{n}(x)$ ein Polynom vom Grad $n \in \mathbb{N}_{0}$ ist.

a) $\underline{\gamma \neq-p}$. Wir suchen eine partikuläre Lösung in der Form

$$
y^{*}(x)=\mathrm{e}^{\gamma \cdot x} \cdot Q_{n}(x)
$$

wobei $Q_{n}(x)$ ein Polynom vom Grad n mit unbekannten Koeffizienten ist.

Beispiel 1.4.2: $\quad y^{\prime}(x)-5 y(x)=x^{2}+3 x$,

Beispiel 1.4.3: $y^{\prime}(x)+2 y(x)=\mathrm{e}^{2 x} \cdot(x+1)$. 
b) $\underline{\gamma=-p}$. Wir suchen eine partikuläre Lösung in der Form

$$
y^{*}(x)=x \cdot \mathrm{e}^{\gamma \cdot x} \cdot Q_{n}(x)
$$

wobei $Q_{n}(x)$ ein Polynom vom Grad n mit unbekannten Koeffizienten ist.

Beispiel 1.4.4: $y^{\prime}(x)+3 y(x)=\mathrm{e}^{-3 x} \cdot(x-1)$.

ii) $f(x):=\mathrm{e}^{\gamma \cdot x} \cdot\left(P_{n}(x) \cdot \cos (\delta \cdot x)+Q_{m}(x) \cdot \sin (\delta \cdot x)\right)$, wobei $\gamma \in \mathbb{R}$ und $\delta \in \mathbb{R} \backslash\{0\}$. Dabei sind $P_{n}(x), Q_{m}(x)$ Polynome vom Grad $n, m \in \mathbb{N}_{0}$ oder genau ein Polynom ist das Nullpolynom.

Wir suchen eine partikuläre Lösung in der Form

$$
y^{*}(x)=\mathrm{e}^{\gamma \cdot x} \cdot\left(S_{N}(x) \cdot \cos (\delta \cdot x)+T_{N}(x) \cdot \sin (\delta \cdot x)\right),
$$

wobei $S_{N}(x), T_{N}(x)$ Polynome vom Grad nicht mehr als $N:=\max \{n, m\}$ mit unbekannten Koeffizienten sind.

Beispiel 1.4.5: $\quad y^{\prime}(x)-2 y(x)=\sin (3 x)+x \cdot \cos (3 x)$,

Beispiel 1.4.6: $\quad y^{\prime}(x)+4 y(x)=\mathrm{e}^{x} \cdot \cos (2 x)$.

\section{Beispiel 1.4.2}

$$
y^{\prime}(x)-5 y(x)=x^{2}+3 x, \quad x \in \mathbb{R} .
$$

1) Die entsprechende homogene Differentialgleichung lautet

$$
y^{\prime}(x)-5 y(x)=0
$$

Hier gilt $p=-5$ und nach Bemerkung 1.2.7 ist

$$
\widetilde{y}(x)=c \cdot \mathrm{e}^{5 x}
$$

mit beliebigem $c \in \mathbb{R}$ die allgemeine Lösung der entsprechenden homogenen Differentialgleichung (1.4.2h) auf $\mathbb{R}$.

2) Wir haben $\gamma=0 \neq 5=-p$, sowie $P_{2}(x)=x^{2}+3 x$ und suchen nach Bemerkung 1.4.1 eine partikuläre Lösung der inhomogenen Gleichung in der Form

$$
y^{*}(x)=A \cdot x^{2}+B \cdot x+D, \quad \text { mit } A, B, D \in \mathbb{R} .
$$


Wir haben

$$
\left(y^{*}\right)^{\prime}(x)=2 A \cdot x+B
$$

und ein Einsetzen in die inhomogene Gleichung (1.4.2) ergibt:

$$
\begin{aligned}
& (2 A \cdot x+B)-5 \cdot\left(A \cdot x^{2}+B \cdot x+D\right)=x^{2}+3 x \\
\Leftrightarrow \quad & -5 A \cdot x^{2}+(2 A-5 B) \cdot x+(B-5 D)=x^{2}+3 x .
\end{aligned}
$$

Ein Koeffizientenvergleich liefert:

$$
\left\{\begin{array} { l } 
{ - 5 A = 1 } \\
{ 2 A - 5 B = 3 } \\
{ B - 5 D = 0 }
\end{array} \Leftrightarrow \left\{\begin{array}{l}
A=-\frac{1}{5} \\
B=-\frac{17}{25} \\
D=-\frac{17}{125}
\end{array}\right.\right.
$$

Dann ist

$$
y^{*}(x)=-\frac{x^{2}}{5}-\frac{17 x}{25}-\frac{17}{125}
$$

eine partikuläre Lösung der inhomogenen Gleichung (1.4.2). Nach Satz 1.3.1 und Folgerung 1.3.2 ist

$$
y(x)=\widetilde{y}(x)+y^{*}(x)=c \cdot \mathrm{e}^{5 x}+\left(-\frac{x^{2}}{5}-\frac{17 x}{25}-\frac{17}{125}\right) \quad \text { mit } \quad c \in \mathbb{R}
$$

die allgemeine Lösung der inhomogenen Gleichung (1.4.2) auf $\mathbb{R}$.

\section{Beispiel 1.4.3}

$$
y^{\prime}(x)+2 y(x)=\mathrm{e}^{2 x} \cdot(x+1), \quad x \in \mathbb{R} .
$$

1) Die entsprechende homogene Differentialgleichung lautet

$$
y^{\prime}(x)+2 y(x)=0 .
$$

Hier gilt $p=2$ und nach Bemerkung 1.2.7 ist

$$
\widetilde{y}(x)=c \cdot \mathrm{e}^{-2 x}
$$

mit beliebigem $c \in \mathbb{R}$ die allgemeine Lösung der entsprechenden homogenen Differentialgleichung (1.4.3h) auf $\mathbb{R}$. 
2) Wir haben $\gamma=2 \neq-2=-p$, sowie $P_{1}(x)=x+1$ und suchen nach Bemerkung 1.4.1 eine partikuläre Lösung der inhomogenen Gleichung in der Form

$$
y^{*}(x)=\mathrm{e}^{2 x} \cdot(A \cdot x+B), \quad \text { mit } A, B \in \mathbb{R} .
$$

Wir haben

$$
\left(y^{*}\right)^{\prime}(x)=2 \cdot \mathrm{e}^{2 x} \cdot(A \cdot x+B)+\mathrm{e}^{2 x} \cdot A=\mathrm{e}^{2 x} \cdot(2 A \cdot x+(A+2 B))
$$

und ein Einsetzen in die inhomogene Gleichung (1.4.3) ergibt:

$$
\begin{array}{rlrl} 
& \mathrm{e}^{2 x} \cdot(2 A \cdot x+(A+2 B))+2 \cdot \mathrm{e}^{2 x} \cdot(A \cdot x+B) & =\mathrm{e}^{2 x} \cdot(x+1) \\
& \Longleftrightarrow & 2 A \cdot x+(A+2 B)+2 A \cdot x+2 B & =x+1 \\
\mathrm{e}^{2 x} \neq 0 & & 4 A \cdot x+(A+4 B) & =x+1 .
\end{array}
$$

Ein Koeffizientenvergleich liefert:

$$
\left\{\begin{array} { l } 
{ 4 A = 1 } \\
{ A + 4 B = 1 }
\end{array} \Leftrightarrow \left\{\begin{array}{l}
A=\frac{1}{4} \\
B=\frac{3}{16}
\end{array}\right.\right.
$$

Dann ist

$$
y^{*}(x)=\mathrm{e}^{2 x} \cdot\left(\frac{x}{4}+\frac{3}{16}\right)
$$

eine partikuläre Lösung der inhomogenen Gleichung (1.4.3). Nach Satz 1.3.1 und Folgerung 1.3.2 ist

$$
y(x)=\widetilde{y}(x)+y^{*}(x)=c \cdot \mathrm{e}^{-2 x}+\mathrm{e}^{2 x} \cdot\left(\frac{x}{4}+\frac{3}{16}\right) \quad \text { mit } \quad c \in \mathbb{R}
$$

die allgemeine Lösung der inhomogenen Gleichung (1.4.3) auf $\mathbb{R}$.

\section{Beispiel 1.4.4}

$$
y^{\prime}(x)+3 y(x)=\mathrm{e}^{-3 x} \cdot(x-1), \quad x \in \mathbb{R} .
$$

1) Die entsprechende homogene Differentialgleichung lautet

$$
y^{\prime}(x)+3 y(x)=0
$$


Hier gilt $p=3$ und nach Bemerkung 1.2.7 ist

$$
\widetilde{y}(x)=c \cdot \mathrm{e}^{-3 x}
$$

mit beliebigem $c \in \mathbb{R}$ die allgemeine Lösung der entsprechenden homogenen Differentialgleichung (1.4.4h) auf $\mathbb{R}$.

2) Wir haben $\gamma=-3=-p$, sowie $P(x)=x-1$ und suchen nach Bemerkung 1.4.1 eine partikuläre Lösung der inhomogenen Gleichung in der Form

$$
y^{*}(x)=x \cdot \mathrm{e}^{-3 x} \cdot(A \cdot x+B)=\mathrm{e}^{-3 x} \cdot\left(A \cdot x^{2}+B \cdot x\right), \quad \text { mit } A, B \in \mathbb{R} .
$$

Wir haben

$$
\begin{aligned}
\left(y^{*}\right)^{\prime}(x) & =-3 \cdot \mathrm{e}^{-3 x} \cdot\left(A \cdot x^{2}+B \cdot x\right)+\mathrm{e}^{-3 x} \cdot(2 A \cdot x+B) \\
& =\mathrm{e}^{-3 x} \cdot\left(-3 A \cdot x^{2}+(2 A-3 B) \cdot x+B\right)
\end{aligned}
$$

und ein Einsetzen in die inhomogene Gleichung (1.4.4) ergibt:

$$
\begin{aligned}
\mathrm{e}^{-3 x} \cdot\left(-3 A \cdot x^{2}+\right. & (2 A-3 B) \cdot x+B) \\
+3 \mathrm{e}^{-3 x} \cdot\left(A \cdot x^{2}+B \cdot x\right) & =\mathrm{e}^{-3 x} \cdot(x-1) \\
2 A \cdot x+B & =x-1 .
\end{aligned}
$$

Ein Koeffizientenvergleich liefert:

$$
\left\{\begin{array} { l } 
{ 2 A = 1 } \\
{ B = - 1 }
\end{array} \Leftrightarrow \left\{\begin{array}{l}
A=\frac{1}{2} \\
B=-1
\end{array}\right.\right.
$$

Dann ist

$$
y^{*}(x)=\mathrm{e}^{-3 x} \cdot\left(\frac{x^{2}}{2}-x\right)
$$

eine partikuläre Lösung der inhomogenen Gleichung (1.4.4). Nach Satz 1.3.1 und Folgerung 1.3.2 ist

$$
y(x)=\widetilde{y}(x)+y^{*}(x)=c \cdot \mathrm{e}^{-3 x}+\mathrm{e}^{-3 x} \cdot\left(\frac{x^{2}}{2}-x\right) \quad \text { mit } \quad c \in \mathbb{R}
$$

die allgemeine Lösung dieser inhomogenen Gleichung (1.4.4) auf $\mathbb{R}$. 


\section{Beispiel 1.4.5}

$$
y^{\prime}(x)-2 y(x)=x \cdot \cos (3 x)+\sin (3 x), \quad x \in \mathbb{R} .
$$

1) Die entsprechende homogene Differentialgleichung lautet

$$
y^{\prime}(x)-2 y(x)=0
$$

Hier gilt $p=-2$ und nach Bemerkung 1.2.7 ist

$$
\widetilde{y}(x)=c \cdot \mathrm{e}^{2 x}
$$

mit beliebigem $c \in \mathbb{R}$ die allgemeine Lösung der entsprechenden homogenen Differentialgleichung (1.4.5h) auf $\mathbb{R}$.

2) Wir haben $\gamma=0, \delta=3, P_{1}(x)=x$ und $Q_{0}(x)=1$ und suchen nach Bemerkung 1.4.1 eine partikuläre Lösung der inhomogenen Gleichung in der Form

$$
y^{*}(x)=(A \cdot x+B) \cdot \cos (3 x)+(D \cdot x+E) \cdot \sin (3 x),
$$

mit $A, B, D, E \in \mathbb{R}$. Wir haben

$$
\begin{gathered}
\left(y^{*}\right)^{\prime}(x)=A \cdot \cos (3 x)+D \cdot \sin (3 x)+(D \cdot x+E) \cdot 3 \cos (3 x) \\
=(3 D \cdot x+(A+3 E)) \cdot \cos (3 x) \\
+(-3 A \cdot x+(D-3 B)) \cdot \sin (3 x)
\end{gathered}
$$

und ein Einsetzen in die inhomogene Gleichung (1.4.5) ergibt:

$$
\begin{gathered}
(3 D \cdot x+(A+3 E)) \cdot \cos (3 x)+(-3 A \cdot x+(D-3 B)) \cdot \sin (3 x) \\
-2 \cdot((A \cdot x+B) \cdot \cos (3 x)+(D \cdot x+E) \cdot \sin (3 x)) \\
=\sin (3 x)+x \cdot \cos (3 x) \\
\Leftrightarrow \quad(3 D-2 A) \cdot x \cdot \cos (3 x)+(A-2 B+3 E) \cdot \cos (3 x) \\
\quad+(-3 A-2 D) \cdot x \cdot \sin (3 x)+(D-3 B-2 E) \cdot \sin (3 x) \\
=\sin (3 x)+x \cdot \cos (3 x) .
\end{gathered}
$$


Ein Koeffizientenvergleich liefert:

$$
\left\{\begin{array} { l } 
{ - 2 A + 3 D = 1 } \\
{ - 3 A - 2 B = 0 } \\
{ A - 2 B + 3 E = 0 } \\
{ D - 3 B - 2 E = 1 }
\end{array} \Leftrightarrow \left\{\begin{array}{l}
A=-\frac{2}{13} \\
D=\frac{3}{13} \\
B=-\frac{34}{169} \\
E=-\frac{14}{169}
\end{array}\right.\right.
$$

Dann ist

$$
y^{*}(x)=\left(-\frac{2}{13} \cdot x-\frac{34}{169}\right) \cdot \cos (3 x)+\left(\frac{3}{13} \cdot x-\frac{14}{169}\right) \cdot \sin (3 x)
$$

eine partikuläre Lösung der inhomogenen Gleichung (1.4.5). Nach Satz 1.3.1 und Folgerung 1.3.2 ist

$$
\begin{aligned}
y(x) & =\widetilde{y}(x)+y^{*}(x) \\
& =c \cdot \mathrm{e}^{2 x}+\left(-\frac{2}{13} \cdot x-\frac{34}{169}\right) \cdot \cos (3 x)+\left(\frac{3}{13} \cdot x-\frac{14}{169}\right) \cdot \sin (3 x)
\end{aligned}
$$

mit $c \in \mathbb{R}$ die allgemeine Lösung der inhomogenen Gleichung (1.4.5) auf $\mathbb{R}$.

\section{Beispiel 1.4.6}

$$
y^{\prime}(x)+4 y(x)=\mathrm{e}^{x} \cdot \cos (2 x), \quad x \in \mathbb{R} .
$$

1) Die entsprechende homogene Differentialgleichung lautet

$$
y^{\prime}(x)+4 y(x)=0
$$

Hier gilt $p=4$ und nach Bemerkung 1.2.7 ist

$$
\widetilde{y}(x)=c \cdot \mathrm{e}^{-4 x}
$$

mit beliebigem $c \in \mathbb{R}$ die allgemeine Lösung der entsprechenden homogenen Differentialgleichung (1.4.6h) auf $\mathbb{R}$. 
2) Wir haben $\gamma=1, \delta=2, P_{0}(x)=1$ und $Q(x) \equiv 0$ (Nullpolynom) und suchen nach Bemerkung 1.4.1 eine partikuläre Lösung der inhomogenen Gleichung in der Form

$$
y^{*}(x)=\mathrm{e}^{x} \cdot(A \cdot \cos (2 x)+B \cdot \sin (2 x)), \quad \text { mit } A, B \in \mathbb{R} .
$$

Wir haben

$$
\begin{aligned}
\left(y^{*}\right)^{\prime}(x)= & \mathrm{e}^{x} \cdot(A \cdot \cos (2 x)+B \cdot \sin (2 x)) \\
& \quad+\mathrm{e}^{x} \cdot(-2 A \cdot \sin (2 x)+2 B \cdot \cos (2 x)) \\
= & \mathrm{e}^{x} \cdot((A+2 B) \cdot \cos (2 x)+(-2 A+B) \cdot \sin (2 x))
\end{aligned}
$$

und ein Einsetzen in die inhomogene Gleichung (1.4.6) ergibt:

$$
\begin{aligned}
& \mathrm{e}^{x} \cdot((A+2 B) \cdot \cos (2 x)+(-2 A+B) \cdot \sin (2 x)) \\
& +4 \mathrm{e}^{x} \cdot(A \cdot \cos (2 x)+B \cdot \sin (2 x))=\mathrm{e}^{x} \cdot \cos (2 x) \\
& \underset{\mathrm{e}^{x} \neq 0}{\Longleftrightarrow}(A+2 B) \cdot \cos (2 x)+(-2 A+B) \cdot \sin (2 x) \\
& +4 \cdot(A \cdot \cos (2 x)+B \cdot \sin (2 x))=\cos (2 x) \\
& \Longleftrightarrow \quad(5 A+2 B) \cdot \cos (2 x)+(-2 A+5 B) \cdot \sin (2 x)=\cos (2 x) \text {. }
\end{aligned}
$$

Ein Koeffizientenvergleich liefert:

$$
\left\{\begin{array} { l } 
{ 5 A + 2 B = 1 } \\
{ - 2 A + 5 B = 0 }
\end{array} \Leftrightarrow \left\{\begin{array}{l}
A=\frac{5}{29} \\
B=\frac{2}{29}
\end{array}\right.\right.
$$

Dann ist

$$
y^{*}(x)=\mathrm{e}^{x} \cdot\left(\frac{5}{29} \cdot \cos (2 x)+\frac{2}{29} \cdot \sin (2 x)\right)
$$

eine partikuläre Lösung der inhomogenen Gleichung (1.4.6). Nach Satz 1.3.1 und Folgerung 1.3.2 ist

$$
y(x)=\widetilde{y}(x)+y^{*}(x)=c \cdot \mathrm{e}^{-4 x}+\mathrm{e}^{x} \cdot\left(\frac{5}{29} \cdot \cos (2 x)+\frac{2}{29} \cdot \sin (2 x)\right) \quad \text { mit }
$$

mit $c \in \mathbb{R}$ die allgemeine Lösung der inhomogenen Gleichung (1.4.6) auf $\mathbb{R}$. 


\section{Beispiel 1.4.7 (Anfangswertproblem)}

$$
\left\{\begin{aligned}
y^{\prime}(x)-\frac{y(x)}{x} & =-2 \frac{\ln (x)}{x}-\frac{12}{x^{3}}, \quad x \in \mathbb{R}^{>0}, \\
y(1) & =5 .
\end{aligned}\right.
$$

1) Der maximale gemeinsame Definitionsbereich der Funktionen $p, f: D \rightarrow \mathbb{R}$ mit $p(x):=-\frac{1}{x}, f(x):=-2 \frac{\ln (x)}{x}-\frac{12}{x^{3}}$ lautet $D=\mathbb{R}^{>0}$. Der Anfangspunkt $x_{0}=1>0$ liegt also im Definitionsbereich $D$. Die inhomogene Gleichung

$$
y^{\prime}(x)-\frac{y(x)}{x}=-2 \frac{\ln (x)}{x}-\frac{12}{x^{3}}
$$

darf auf $\mathbb{R}^{>0}$ untersucht werden.

2) Die entsprechende homogene Differentialgleichung lautet

$$
y^{\prime}(x)-\frac{y(x)}{x}=0
$$

Es gilt für alle $x \in \mathbb{R}^{>0}$ :

$$
\int p(x) \mathrm{d} x=-\int \frac{1}{x} \mathrm{~d} x=-\ln (x)+m \quad \text { mit } \quad m \in \mathbb{R} .
$$

Wir nehmen (z.B.) $m=0$, dann ist $P(x)=-\ln (x)$ und $y_{1}=\mathrm{e}^{-P(x)}=x$, sowie $\mathrm{e}^{P(x)}=\frac{1}{x}$ auf $\mathbb{R}^{>0}$. Nach Bemerkung 1.2.7 ist

$$
\widetilde{y}(x)=\widetilde{c} \cdot x
$$

mit beliebigem $\widetilde{c} \in \mathbb{R}$ die allgemeine Lösung der entsprechenden homogenen Differentialgleichung $(1.4 .7 \mathrm{~h})$ auf $(0 ; \infty)$.

3) Wir suchen eine partikuläre Lösung der inhomogenen Gleichung

$$
y^{\prime}(x)-\frac{y(x)}{x}=-2 \frac{\ln (x)}{x}
$$

mit dem Verfahren der Variation der Konstanten. Nach Bemerkung 1.3.4 erhalten wir auf $\mathbb{R}^{>0}$ :

$$
c^{\prime}(x)=\frac{1}{x} \cdot\left(-2 \frac{\ln (x)}{x}\right)=-\frac{2 \ln (x)}{x^{2}},
$$


und somit

$$
c(x)=\int \frac{-2 \ln (x)}{x^{2}} \mathrm{~d} x=\left\{\begin{array}{c}
u(x)=\ln (x) \\
u^{\prime}(x)=\frac{1}{x} \\
v^{\prime}(x)=-\frac{2}{x^{2}} \\
v(x)=\frac{2}{x}
\end{array}\right\}=\frac{2 \ln (x)}{x}-\int \frac{2}{x^{2}} \mathrm{~d} x
$$

Mit $n=0$ bekommen wir folgende partikuläre Lösung

$$
y_{1}^{*}(x)=c(x) \cdot y_{1}(x)=\left(\frac{2 \ln (x)}{x}+\frac{2}{x}\right) \cdot x=2 \ln (x)+2
$$

der inhomogenen Differentialgleichung (1.4.7a) auf $(0 ; \infty)$.

4) Wir suchen eine partikuläre Lösung der inhomogenen Gleichung

$$
y^{\prime}(x)-\frac{y(x)}{x}=-\frac{12}{x^{3}}
$$

mit dem Verfahren der Variation der Konstanten. Nach Bemerkung 1.3.4 erhalten wir auf $\mathbb{R}^{>0}$ :

$$
c^{\prime}(x)=\frac{1}{x} \cdot\left(-\frac{12}{x^{3}}\right)=-\frac{12}{x^{4}},
$$

und somit

$$
c(x)=-\int \frac{12}{x^{4}} \mathrm{~d} x=\frac{4}{x^{3}}+n, \quad n \in \mathbb{R} .
$$

Mit $n=0$ bekommen wir folgende partikuläre Lösung

$$
y_{2}^{*}(x)=c(x) \cdot y_{1}(x)=\frac{4}{x^{2}}
$$

der inhomogenen Differentialgleichung (1.4.7b) auf $(0 ; \infty)$.

5) Nach dem Superpositionsprinzip 1.3.7 ist

$$
y^{*}(x)=y_{1}^{*}(x)+y_{2}^{*}(x)
$$


eine partikuläre Lösung der inhomogenen Differentialgleichung

$$
y^{\prime}(x)-\frac{y(x)}{x}=-2 \frac{\ln (x)}{x}-\frac{12}{x^{3}}
$$

auf $\mathbb{R}^{>0}$ und

$$
y(x)=\widetilde{y}(x)+y^{*}(x)=\widetilde{c} \cdot x+2 \ln (x)+2+\frac{4}{x^{2}}
$$

die allgemeine Lösung dieser inhomogenen Gleichung auf $\mathbb{R}^{>0}$.

6) Mit Hilfe des Anfangswertes $y(1)=5$ bekommen wir:

$$
y(1)=5 \quad \Leftrightarrow \quad \widetilde{c} \cdot 1+2 \ln (1)+2+\frac{4}{1^{2}}=5 \quad \Leftrightarrow \quad \widetilde{c}=-1
$$

Dann ist

$$
y(x)=-x+2 \ln (x)+2+\frac{4}{x^{2}}
$$

die eindeutige Lösung des Anfangswertproblems (1.4.7) auf $(0 ; \infty)$.

\subsection{Auf- und Entladung eines Kondensators}

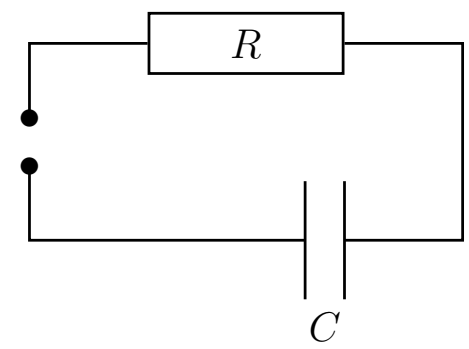

Wir untersuchen einen Stromkreis, bestehend aus einem Ohm'schen Widerstand $^{1} R>0$ und einem Kondensator mit der Kapazität $C>0$.

\subsubsection{Aufladung eines Kondensators}

Die Stromquelle wird zum Zeitpunkt $t_{0}=0$ eingeschaltet und mit einer konstanten Spannung $U_{0}$ betrieben. Der Kondensator fäng an sich aufzuladen.

Nach dem zweiten KIRCHHOFF'schen Gesetz ${ }^{2}$ gilt

$$
U_{0}=U_{R}(t)+U_{C}(t), \quad t \in \mathbb{R}^{\geqslant 0} .
$$

\footnotetext{
${ }^{1}$ Georg Simon Ohm, 1789-1854

${ }^{2}$ Gustav Robert Kirchhoff, 1824-1887
} 
Wobei wir mit $U_{R}(t)$ die Spannung am Widerstand und mit $U_{C}(t)$ die Spannung am Kondensator bezeichnen. Es gilt $(C>0)$ :

$$
U_{R}(t)=R \cdot I(t), \quad U_{C}(t)=\frac{Q(t)}{C}, \quad I(t)=\dot{Q}(t),
$$

wobei $I:=I(t)$ Stromstärke im Kreis und $Q:=Q(t)$ Ladung des Kondensators sind. Dann

$U_{R}(t)+U_{C}(t)=U_{0} \quad \Leftrightarrow \quad R \cdot \dot{Q}(t)+\frac{Q(t)}{C}=U_{0} \quad \underset{R>0}{\Leftrightarrow} \quad \dot{Q}(t)+\frac{Q(t)}{R \cdot C}=\frac{U_{0}}{R}$,

d.h., die Ladung am Kondensator genügt einer linearen inhomogenen Differentialgleichung erster Ordnung mit konstantem Koeffizienten auf $\mathbb{R}^{\geqslant 0}$. Die entsprechende homogene Differentialgleichung lautet

$$
\dot{Q}(t)+\frac{Q(t)}{R \cdot C}=0
$$

Nach Bemerkung 1.2.7 ist

$$
\widetilde{Q}(t)=\widetilde{c} \cdot \mathrm{e}^{-\frac{t}{R \cdot C}}
$$

mit beliebigem $\widetilde{c} \in \mathbb{R}$ die allgemeine Lösung der homogenen Differentialgleichung auf $\mathbb{R}^{\geqslant 0}$. Nach Bemerkung 1.4.1 suchen wir nun eine partikuläre Lösung der inhomogenen Gleichung in der Form

$$
Q^{*}(t)=A \quad \text { mit } A \in \mathbb{R}, \text { dann ist } \quad \dot{Q}^{*}(t)=0
$$

und ein Einsetzen in die inhomogene Gleichung ergibt:

$$
0+\frac{A}{R \cdot C}=\frac{U_{0}}{R} \underset{R, C>0}{\Longleftrightarrow} \quad A=U_{0} \cdot C
$$

Dann ist $Q^{*}(t)=U_{0} \cdot C$ eine partikuläre Lösung und

$$
Q(t)=\widetilde{Q}(t)+Q^{*}(t)=\widetilde{c} \cdot \mathrm{e}^{-\frac{t}{R \cdot C}}+U_{0} \cdot C
$$

die allgemeine Lösung der inhomogenen Gleichung auf $\mathbb{R}^{\geqslant 0}$.

Mit der Anfangsbedingung $Q(0)=0$ erhalten wir

$\widetilde{c}+U_{0} \cdot C=0 \Leftrightarrow \widetilde{c}=-U_{0} \cdot C \quad$ und somit $\quad Q(t)=U_{0} \cdot C \cdot\left(1-\mathrm{e}^{-\frac{t}{R \cdot C}}\right)$. 
Für die Kondensatorspannung bekommen wir

$$
U_{C}(t)=\frac{Q(t)}{C}=U_{0} \cdot\left(1-\mathrm{e}^{-\frac{t}{R \cdot C}}\right)
$$

und für die Stromstärke

$$
I(t)=\dot{Q}(t)=\frac{U_{0}}{R} \cdot \mathrm{e}^{-\frac{t}{R \cdot C}} .
$$

Bezeichnen wir die Stromstärke am Anfang durch

$$
I_{0}:=I(0)=\frac{U_{0}}{R}, \quad \text { dann gilt } \quad I(t)=I_{0} \cdot \mathrm{e}^{-\frac{t}{R \cdot C}},
$$

wir sprechen von einem Ladestrom.
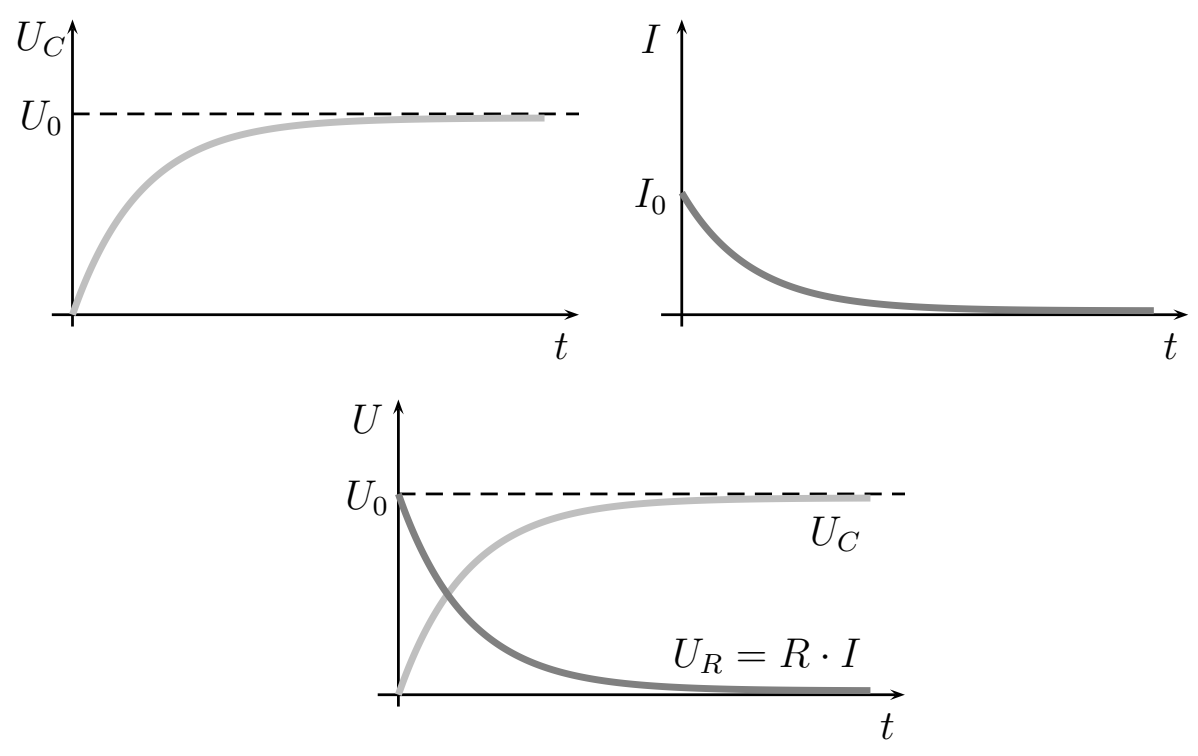

Für die Widerstandspannung gilt:

$$
U_{R}(t)=R \cdot I(t) \cdot \mathrm{e}^{-\frac{t}{R \cdot C}} .
$$

Beachte: Es gilt stets $U_{R}(t)+U_{C}(t)=U_{0}$ zu jeder Zeit $t \geqslant 0$.

\subsubsection{Entladung eines Kondensators}

Die Stromquelle wird zum Zeitpunkt $t_{0}=0$ abgeschaltet. Der Kondensator beginnt sich zu entladen. Nach dem zweiten KIRCHноFF'schen Gesetz gilt:

$$
U_{R}(t)+U_{C}(t)=0, \quad t \in \mathbb{R}^{\geqslant 0} .
$$


Mit $U_{R}(t)=R \cdot I(t), U_{C}(t)=\frac{Q(t)}{C}, I(t)=\dot{Q}(t)$ (wobei $\left.R, C>0\right)$ folgt

$$
U_{R}(t)+U_{C}(t)=0 \quad \Leftrightarrow \quad R \cdot \dot{Q}(t)+\frac{Q(t)}{C}=0 \quad \Leftrightarrow \quad \dot{Q}(t)+\frac{Q(t)}{R \cdot C}=0
$$

d.h., die Ladung am Kondensator genügt einer linearen homogenen Differentialgleichung erster Ordnung mit konstantem Koeffizienten auf $\mathbb{R}^{\geqslant 0}$. Nach Bemerkung 1.2.7 ist

$$
Q(t)=\widetilde{c} \cdot \mathrm{e}^{-\frac{t}{R \cdot C}}
$$

mit beliebigem $\widetilde{c} \in \mathbb{R}$ die allgemeine Lösung der homogenen Differentialgleichung auf $\mathbb{R}^{\geqslant 0}$. Wenn $Q(0)=Q_{0}$ die Anfangsbedingung ist, dann ist $\widetilde{c}=Q_{0}$ und somit

$$
Q(t)=Q_{0} \cdot \mathrm{e}^{-\frac{t}{R \cdot C}}
$$

Für die Kondensatorspannung bekommen wir

$$
U_{C}(t)=\frac{Q(t)}{C}=\frac{Q_{0}}{C} \cdot \mathrm{e}^{-\frac{t}{R \cdot C}}
$$

und für die Stromstärke

$$
I(t)=\dot{Q}(t)=-\frac{Q_{0}}{R \cdot C} \cdot \mathrm{e}^{-\frac{t}{R \cdot C}}
$$

Bezeichnen wir mit $\quad U_{0}:=\frac{Q_{0}}{C} \quad$ und mit $\quad I_{0}:=-\frac{U_{0}}{R}, \quad$ dann gilt

$$
U_{C}(t)=U_{0} \cdot \mathrm{e}^{-\frac{t}{R \cdot C}} \quad \text { und } \quad I(t)=I_{0} \cdot \mathrm{e}^{-\frac{t}{R \cdot C}}
$$

(sog. Entladestrom).
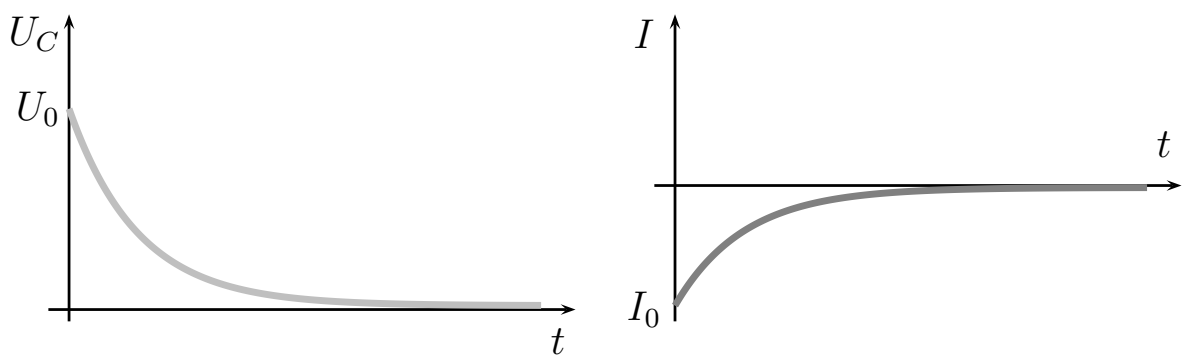


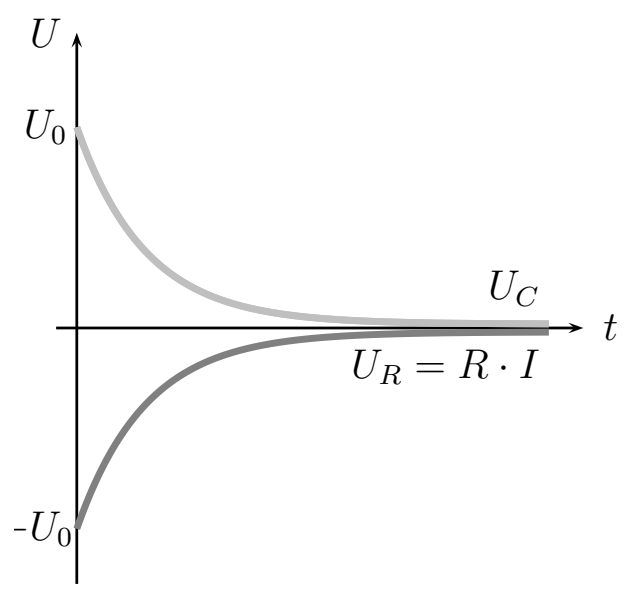

Für die Widerstandspannung gilt

$$
U_{R}(t)=R \cdot I(t)=-\frac{Q_{0}}{C} \cdot \mathrm{e}^{-\frac{t}{R \cdot C}}=-U_{0} \cdot \mathrm{e}^{-\frac{t}{R \cdot C}} .
$$

Beachte: Es gilt stets $U_{R}(t)+U_{C}(t)=0$ zu jeder Zeit $t \geqslant 0$.

Der Wert $\tau:=R \cdot C$ heißt Zeitkonstante, dann ist $\quad \mathrm{e}^{-\frac{t}{R \cdot C}}=\mathrm{e}^{-\frac{t}{\tau}}$.

\subsection{Ein- und Ausschalten eines Stromkreises mit Spule und OHM'schem Widerstand}

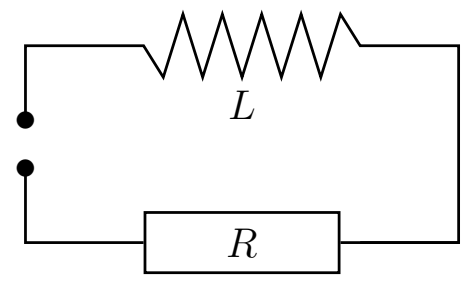

Wir untersuchen einen Stromkreis, bestehend aus einem OHM'schen Widerstand $R>0$ und einer Spule mit der Induktivität $L>0$.

\subsubsection{Einschalten des Stroms}

Die Stromquelle wird zum Zeitpunkt $t_{0}=0$ eingeschaltet und mit einer konstanten Spannung $U_{0}$ betrieben. Nach dem zweiten KirchHofF'schen Gesetz gilt:

$$
U_{0}=U_{R}(t)+U_{L}(t), \quad t \in \mathbb{R}^{\geqslant 0},
$$


wobei wir mit $U_{R}$ die Spannung am Widerstand und mit $U_{L}$ die Spannung an der Spule bezeichnen. Es gilt:

$$
U_{R}(t)=R \cdot I(t), \quad U_{L}(t)=L \cdot \dot{I}(t),
$$

wobei $I:=I(t)$ die Stromstärke im Kreis ist. Dann

$U_{R}(t)+U_{L}(t)=U_{0} \quad \Leftrightarrow \quad R \cdot I(t)+L \cdot \dot{I}(t)=U_{0} \quad \underset{L>0}{\Leftrightarrow} \quad \dot{I}(t)+\frac{R}{L} \cdot I(t)=\frac{U_{0}}{L}$,

d.h., die Stromstärke genügt einer linearen inhomogenen Differentialgleichung erster Ordnung mit konstantem Koeffizienten auf $\mathbb{R}^{\geqslant 0}$. Die entsprechende homogene Differentialgleichung lautet

$$
\dot{I}(t)+\frac{R}{L} \cdot I(t)=0 .
$$

Nach Bemerkung 1.2.7 ist

$$
\widetilde{I}(t)=\widetilde{c} \cdot \mathrm{e}^{-\frac{R}{L} \cdot t}
$$

mit beliebigem $\widetilde{c} \in \mathbb{R}$ die allgemeine Lösung der homogenen Differentialgleichung auf $\mathbb{R}^{\geqslant 0}$.

Nach Bemerkung 1.4.1 suchen wir eine partikuläre Lösung der inhomogenen Gleichung der Form

$$
I^{*}(t)=A, \quad \text { mit } A \in \mathbb{R}, \text { dann ist } \quad \dot{I}^{*}(t)=0,
$$

und ein Einsetzen in die inhomogene Gleichung ergibt

$$
0+\frac{R}{L} \cdot A=\frac{U_{0}}{L} \quad \underset{R, L>0}{\Longleftrightarrow} \quad A=\frac{U_{0}}{R}
$$

Dann ist $I^{*}(t)=\frac{U_{0}}{R}$ eine partikuläre und

$$
I(t)=\widetilde{I}(t)+I^{*}(t)=\widetilde{c} \cdot \mathrm{e}^{-\frac{R}{L} \cdot t}+\frac{U_{0}}{R}
$$

die allgemeine Lösung der inhomogenen Gleichung auf $\mathbb{R}^{\geqslant 0}$. Mit der Anfangsbedingung $I(0)=0$ erhalten wir

$$
\widetilde{c}+\frac{U_{0}}{R}=0 \quad \Leftrightarrow \quad \widetilde{c}=-\frac{U_{0}}{R} \quad \text { und somit } \quad I(t)=\frac{U_{0}}{R} \cdot\left(1-\mathrm{e}^{-\frac{R}{L} \cdot t}\right) .
$$


Für die Widerstandspannung bekommen wir

$$
U_{R}(t)=R \cdot I(t)=U_{0} \cdot\left(1-\mathrm{e}^{-\frac{R}{L} \cdot t}\right) .
$$

Es gilt

$$
\dot{I}(t)=\frac{U_{0}}{R} \cdot \mathrm{e}^{-\frac{R}{L} \cdot t}
$$

und für die Spulenspannung folgt

$$
U_{L}(t)=L \cdot I(t)=U_{0} \cdot \mathrm{e}^{-\frac{R}{L} \cdot t} .
$$

Beachte: Es gilt stets $U_{R}(t)+U_{L}(t)=U_{0}$ zu jeder Zeit $t \geqslant 0$.
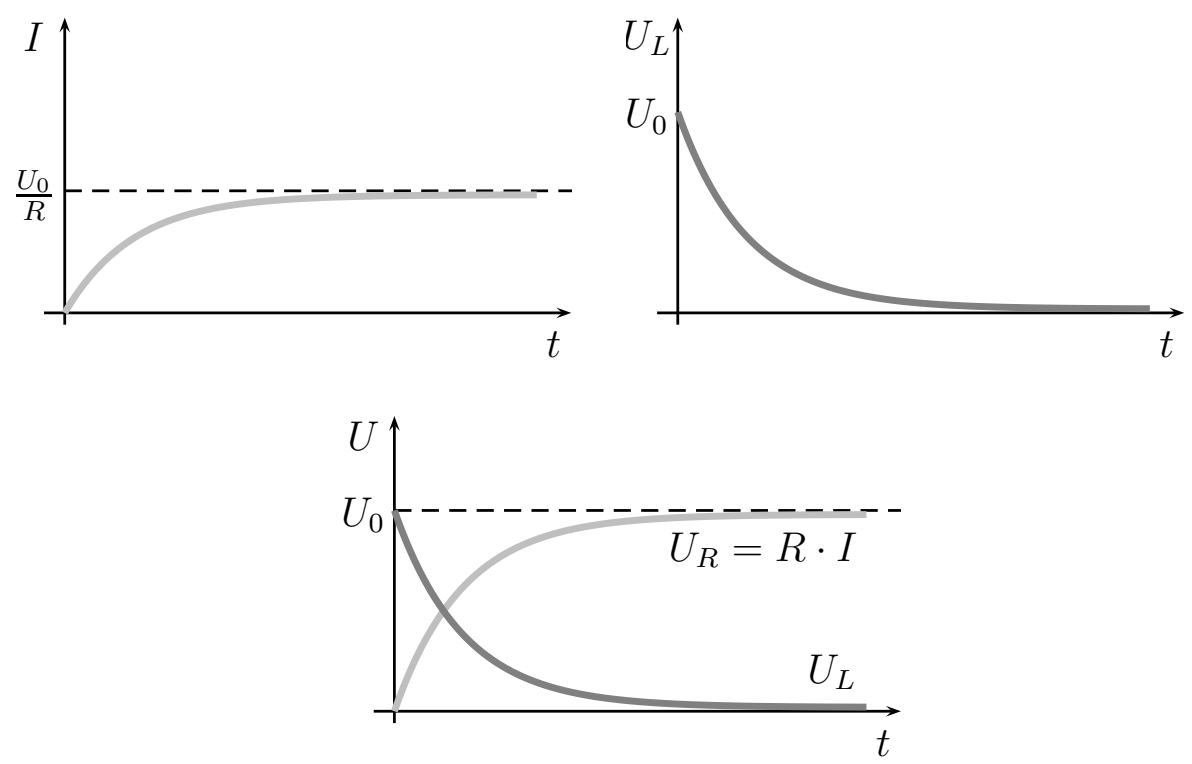

\subsubsection{Ausschalten des Stroms}

Die Stromquelle wird zum Zeitpunkt $t_{0}=0$ ausgeschaltet. Nach dem zweiten KIRCHHOFF'schen Gesetz gilt:

$$
U_{R}(t)+U_{L}(t)=0, \quad t \in \mathbb{R}^{\geqslant 0}
$$

$\operatorname{mit} U_{R}(t)=R \cdot I(t)$ und $U_{L}(t)=L \cdot \dot{I}(t)$. Somit

$U_{R}(t)+U_{L}(t)=0 \quad \Leftrightarrow \quad R \cdot I(t)+L \cdot \dot{I}(t)=0 \quad \underset{L>0}{\Leftrightarrow} \quad \dot{I}(t)+\frac{R}{L} \cdot I(t)=0$, 
d.h., die Stromstärke genügt einer linearen inhomogenen Differentialgleichung erster Ordnung mit konstantem Koeffizienten auf $\mathbb{R}^{\geqslant 0}$. Nach Bemerkung 1.2.7 ist

$$
I(t)=\widetilde{c} \cdot \mathrm{e}^{-\frac{R}{L} \cdot t}
$$

mit beliebigem $\widetilde{c} \in \mathbb{R}$ die allgemeine Lösung der homogenen Differentialgleichung auf $\mathbb{R}^{\geqslant 0}$. Mit der Anfangsbedingung $I(0)=I_{0}$ folgt $\widetilde{c}=I_{0}$ und somit

$$
I(t)=I_{0} \cdot \mathrm{e}^{-\frac{R}{L} \cdot t}
$$

Für die Widerstandspannung bekommen wir

$$
U_{R}(t)=R \cdot I(t)=R \cdot I_{0} \cdot \mathrm{e}^{-\frac{R}{L} \cdot t}
$$

Bezeichnen wir mit $U_{0}:=R \cdot I_{0}$ dann gilt

$$
U_{R}(t)=U_{0} \cdot \mathrm{e}^{-\frac{R}{L} \cdot t}
$$

Ferner ist

$$
\dot{I}(t)=I_{0} \cdot\left(-\frac{R}{L}\right) \cdot \mathrm{e}^{-\frac{R}{L} \cdot t}=-\frac{U_{0}}{L} \cdot \mathrm{e}^{-\frac{R}{L} \cdot t},
$$

sodass für die Spulenspannung folgt

$$
U_{L}(t)=L \cdot I(t)=-U_{0} \cdot \mathrm{e}^{-\frac{R}{L} \cdot t}
$$

Beachte: Es gilt stets $U_{R}(t)+U_{L}(t)=0$ zu jeder Zeit $t \geqslant 0$.
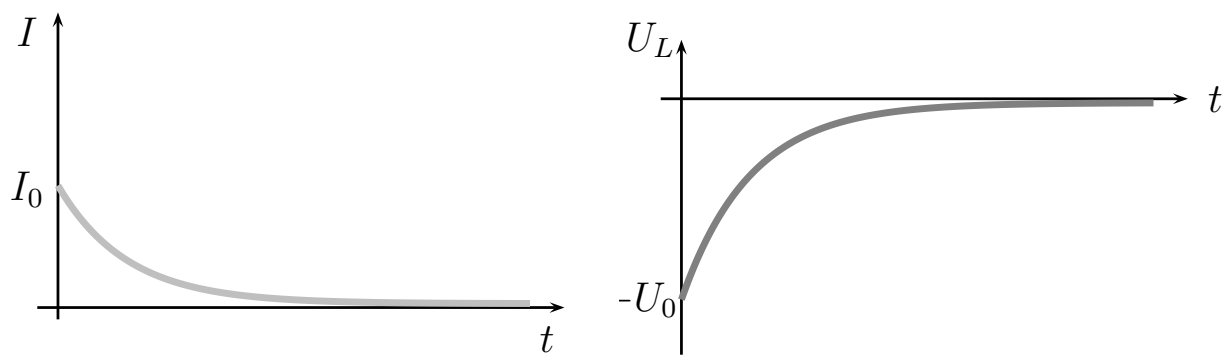


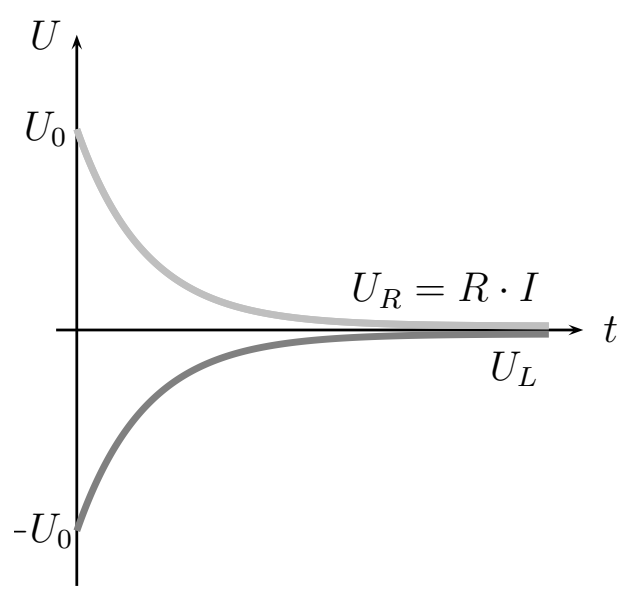

Der Wert $\tau:=\frac{L}{R}$ heißt Zeitkonstante, dann ist $\quad \mathrm{e}^{-\frac{R}{L} \cdot t}=\mathrm{e}^{-\frac{t}{\tau}}$. 


\section{Lineare Differenzengleichungen erster Ordnung}

Vorbemerkung: Im Folgenden werden wir Differenzengleichungen auf der Menge $\mathbb{N}_{0}$ untersuchen. Analoge Betrachtungen lassen sich auf $\mathbb{Z}^{\geqslant n_{0}}:=$ $\left\{n_{0}, n_{0}+1, \ldots\right\}$ mit einem beliebigen $n_{0} \in \mathbb{Z}$ oder auf der ganzen Menge $\mathbb{Z}$ durchführen.

\subsection{Anfangswertproblem für lineare Differenzen- gleichungen erster Ordnung}

\section{Definition 2.1.1}

Gegeben seien die Funktionen $p, f: \mathbb{N}_{0} \rightarrow \mathbb{R}$, wobei $p(n) \neq 0$ für alle $n \in \mathbb{N}_{0}$ ist. Gesucht ist eine Funktion $x: \mathbb{N}_{0} \rightarrow \mathbb{R}$, sodass für alle $n \in \mathbb{N}_{0}$ gilt:

$$
x(n+1)+p(n) \cdot x(n)=f(n) .
$$

Gleichung (2.1.1) heißt lineare Differenzengleichung erster Ordnung. Die Funktion $x: \mathbb{N}_{0} \rightarrow \mathbb{R}$ heißt Lösung der Gleichung (2.1.1).

\section{Satz und Definition 2.1.2}

Gegeben seien die Funktionen $p, f: \mathbb{N}_{0} \rightarrow \mathbb{R}$, wobei $p(n) \neq 0$ für alle $n \in \mathbb{N}_{0}$ ist, sowie $n_{0} \in \mathbb{N}_{0}$. Dann existiert für beliebiges $\mu_{0} \in \mathbb{R}$ auf $\mathbb{N}_{0}$ genau eine Lösung des Anfangswertproblems

$$
\left\{\begin{aligned}
x(n+1)+p(n) \cdot x(n) & =f(n), \quad n \in \mathbb{N}_{0}, \\
x\left(n_{0}\right) & =\mu_{0} .
\end{aligned}\right.
$$


BEWEIS:

\section{Existenz:}

Ist der Wert $x\left(n_{0}\right)$ an einer Stelle $n_{0} \in \mathbb{N}_{0}$ gegeben, so ist auch der Wert an der Stelle $\left(n_{0}+1\right)$ bereits bestimmt:

$$
x\left(n_{0}+1\right)=f\left(n_{0}\right)-p\left(n_{0}\right) \cdot x\left(n_{0}\right) .
$$

Für $\left(n_{0}-1\right) \in \mathbb{N}_{0}$ erhalten wir aus der Gültigkeit der Differenzengleichung

$$
x\left(n_{0}-1\right)=\frac{f\left(n_{0}-1\right)-x\left(n_{0}\right)}{p\left(n_{0}-1\right)} .
$$

Sukzessive kann also jeder Wert von $x($.$) auf \mathbb{N}_{0}$ bestimmt werden und es existiert eine Lösung des Anfangswertproblems.

\section{Eindeutigkeit:}

Seien $x_{1}, x_{2}: \mathbb{N}_{0} \rightarrow \mathbb{R}$ zwei Lösungen des Anfangswertproblems (2.1.2).

Dann gilt

$$
\left\{\begin{aligned}
x_{1}(n+1)+p(n) \cdot x_{1}(n) & =f(n), \quad n \in \mathbb{N}_{0}, \\
x_{1}\left(n_{0}\right) & =\mu_{0} .
\end{aligned}\right.
$$

Aber auch

$$
\left\{\begin{aligned}
x_{2}(n+1)+p(n) \cdot x_{2}(n) & =f(n), \quad n \in \mathbb{N}_{0} \\
x_{2}\left(n_{0}\right) & =\mu_{0} .
\end{aligned}\right.
$$

Für $\widetilde{x}(n):=x_{2}(n)-x_{1}(n)$ folgt:

$$
\left\{\begin{aligned}
\widetilde{x}(n+1)+p(n) \cdot \widetilde{x}(n) & =0, \quad n \in \mathbb{N}_{0}, \\
\widetilde{x}\left(n_{0}\right) & =0 .
\end{aligned}\right.
$$

Wir beweisen, dass (2.1.3) nur die triviale Lösung $\widetilde{x}(n)=0$ für alle $n \in \mathbb{N}_{0}$ zulässt:

Wir zeigen zuerst, dass für alle $n \in \mathbb{N}_{0}$ mit $n \geqslant n_{0}$ bereits $\widetilde{x}(n)=0$ gelten muss:

Mit der Anfangsbedingung haben wir schon $\widetilde{x}\left(n_{0}\right)=0$. Angenommen, es existiert ein $n \in \mathbb{N}$ mit $n \geqslant n_{0}+1$, sodass $\widetilde{x}(n) \neq 0$ gilt. Dann ist die Menge $\left\{n \in \mathbb{N}: n \geqslant\left(n_{0}+1\right)\right.$ und $\left.\widetilde{x}(n) \neq 0\right\}$ nichtleer. 
Sei $s:=\min \left\{n \in \mathbb{N}: n \geqslant\left(n_{0}+1\right)\right.$ und $\left.\widetilde{x}(n) \neq 0\right\}$. Dann gilt $\widetilde{x}(s-1)=0$. Nach (2.1.3) folgt $\widetilde{x}(s)=-p(s-1) \cdot \widetilde{x}(s-1)=0$, im Widerspruch zur Auswahl von $s$. Also ist $\widetilde{x}(n)=0$ für alle $n \in \mathbb{N}_{0}$ mit $n \geqslant n_{0}$.

Ist $n_{0}=0$, so folgt somit sofort $\widetilde{x}(n)=0$ für alle $n \in \mathbb{N}_{0}$.

Jetzt zeigen wir, dass auch die Behauptung $\widetilde{x}(n)=0$ für alle $n \in \mathbb{N}_{0}$ mit $n \leqslant\left(n_{0}-1\right)$ folgt:

Andernfalls wäre die Menge $\left\{n \in \mathbb{N}_{0}: n \leqslant\left(n_{0}-1\right)\right.$ und $\left.\widetilde{x}(n) \neq 0\right\}$ nichtleer und es existierte $t:=\max \left\{n \in \mathbb{N}_{0}: n \leqslant\left(n_{0}-1\right)\right.$ und $\left.\widetilde{x}(n) \neq 0\right\}$. Dann gilt $\widetilde{x}(t+1)=0$. Wegen

$$
\underbrace{\widetilde{x}(t+1)}_{=0}+p(t) \cdot \widetilde{x}(t)=0 \quad \text { und } \quad p(t) \neq 0 \quad \text { folgt } \quad \widetilde{x}(t)=0
$$

im Widerspruch zur Auswahl von $t$. Also ist auch $\widetilde{x}(n)=0$ für alle $n \in \mathbb{N}_{0}$ mit $n \leqslant\left(n_{0}-1\right)$.

Wir haben gezeigt, dass das Anfangswertproblem (2.1.3) nur die triviale Lösung $\widetilde{x}(n)=0$ für alle $n \in \mathbb{N}_{0}$ besitzt, d.h., es gilt $x_{1}(n)=x_{2}(n)$ für alle $n \in \mathbb{N}_{0}$. Damit ist die Lösung von (2.1.2) eindeutig bestimmt.

\section{Bemerkung und Definition 2.1.3}

Ist $f(n)=0$ für alle $n \in \mathbb{N}_{0}$, so heißt Gleichung (2.1.1) lineare homogene Differenzengleichung erster Ordnung, andernfalls heißt diese lineare inhomogene Differenzengleichung erster Ordnung.

\subsection{Lineare homogene Differenzengleichung erster Ordnung}

Wir untersuchen zuerst die homogene Gleichung

$$
x(n+1)+p(n) \cdot x(n)=0
$$

(dabei ist die Funktion $p: \mathbb{N}_{0} \rightarrow \mathbb{R}$ mit $p(n) \neq 0$ für alle $n \in \mathbb{N}_{0}$ gegeben). 


\section{Satz 2.2.1}

Sei $x_{1}: \mathbb{N}_{0} \rightarrow \mathbb{R}$ eine Lösung von (2.2.1), dann ist $x(n):=c \cdot x_{1}(n)$ ebenfalls eine Lösung von (2.2.1), wobei $c \in \mathbb{R}$ eine beliebige Konstante ist.

BEwEIS:

Da $x_{1}$ eine Lösung von (2.2.1) ist, gilt für alle $n \in \mathbb{N}_{0}$ :

$$
x_{1}(n+1)+p(n) \cdot x_{1}(n)=0
$$

Dann folgt für $x(n):=c \cdot x_{1}(n)$, wobei $c \in \mathbb{R}$ eine beliebige Konstante ist:

$$
\begin{aligned}
x(n+1)+p(n) \cdot x(n) & =c \cdot x_{1}(n+1)+p(n) \cdot c \cdot x_{1}(n) \\
& =c \cdot\left(x_{1}(n+1)+p(n) \cdot x_{1}(n)\right) \\
& \stackrel{(2.2 .2)}{=} 0 \quad \text { auf } \mathbb{N}_{0} .
\end{aligned}
$$

Nach Definition 2.1.1 ist also $x(n):=c \cdot x_{1}(n)$ eine Lösung von (2.2.1) auf $\mathbb{N}_{0}$, wobei $c \in \mathbb{R}$ eine beliebige Konstante ist.

\section{Bemerkung 2.2.2}

Die Funktion $x_{1}: \mathbb{N}_{0} \rightarrow \mathbb{R}$ mit

$$
x_{1}(n):=(-1)^{n} \cdot \prod_{k=0}^{n-1} p(k)
$$

ist eine Lösung von (2.2.1) auf $\mathbb{N}_{0}$. Dabei gilt für $n=0$ nach Definition des leeren Produktes

$$
x_{1}(0)=(-1)^{0} \cdot 1=1 .
$$

In der Tat gilt für jedes $n \in \mathbb{N}_{0}$

$$
\begin{aligned}
x_{1}(n+1)+p(n) \cdot x_{1}(n) & =(-1)^{n+1} \cdot \prod_{k=0}^{n} p(k)+p(n) \cdot(-1)^{n} \cdot \prod_{k=0}^{n-1} p(k) \\
& =(-1)^{n} \cdot\left(-\prod_{k=0}^{n} p(k)+p(n) \cdot \prod_{k=0}^{n-1} p(k)\right) \\
& =(-1)^{n} \cdot\left(-\prod_{k=0}^{n} p(k)+\prod_{k=0}^{n} p(k)\right)=0 .
\end{aligned}
$$




\section{Satz und Definition 2.2.3}

Allgemeine Lösung der linearen homogenen Differenzengleichung erster Ordnung

fede Lösung $x: \mathbb{N}_{0} \rightarrow \mathbb{R}$ von (2.2.1) lässt sich in der Form $x(n)=c \cdot x_{1}(n)$ darstellen, wobei

$$
x_{1}(n)=(-1)^{n} \cdot \prod_{k=0}^{n-1} p(k)
$$

und der Koeffizient $c \in \mathbb{R}$ eindeutig bestimmt ist. Lässt man in $c \cdot x_{1}(n)$ die Zahl $c$ die Menge der reellen Zahlen durchlaufen, so erhält man alle Lösungen der Gleichung (2.2.1). Man sagt: Durch $x(n)=c \cdot x_{1}(n)$ ist die allgemeine Lösung der linearen homogenen Differenzengleichung erster Ordnung (2.2.2) gegeben.

BEWEIS:

Nach Bemerkung 2.2.2 und Satz 2.2.1 ist jede Funktion $x: \mathbb{N}_{0} \rightarrow \mathbb{R}$ der Form $x(n):=c \cdot x_{1}(n)$ mit

$$
x_{1}(n)=(-1)^{n} \cdot \prod_{k=0}^{n-1} p(k)
$$

immer eine Lösung von (2.2.1) für jedes $c \in \mathbb{R}$.

Sei $\widetilde{x}: \mathbb{N}_{0} \rightarrow \mathbb{R}$ eine beliebige Lösung von (2.2.1). Wir zeigen: Es existiert ein $\widetilde{c} \in \mathbb{R}$, sodass auf $\mathbb{N}_{0}$ gilt: $\quad \widetilde{x}(n)=\widetilde{c} \cdot x_{1}(n)$.

Da $\widetilde{x}$ eine Lösung von (2.2.1) ist, gilt $\widetilde{x}(n+1)+p(n) \cdot \widetilde{x}(n)=0$ auf $\mathbb{N}_{0}$. Die Funktion $z: \mathbb{N}_{0} \rightarrow \mathbb{R}$ mit

$$
z(n):=\frac{\widetilde{x}(n)}{x_{1}(n)}
$$

ist auf $\mathbb{N}_{0}$ definiert, denn $p(n) \neq 0$ für alle $n \in \mathbb{N}_{0}$. Weiter gilt für alle $n \in \mathbb{N}_{0}$ :

$$
z(n+1)=\frac{\widetilde{x}(n+1)}{x_{1}(n+1)}=\frac{-p(n) \cdot \widetilde{x}(n)}{-p(n) \cdot x_{1}(n)}=\frac{\widetilde{x}(n)}{x_{1}(n)}=z(n)
$$

Somit ist $z$ konstant auf $\mathbb{N}_{0}$. Also existiert ein $\widetilde{c} \in \mathbb{R}$ mit $\frac{\widetilde{x}(n)}{x_{1}(n)}=\widetilde{c}$ auf $\mathbb{N}_{0}$. Dann ist jede Lösung von (2.2.1) in der Form $c \cdot x_{1}(n)$ mit

$$
x_{1}(n)=(-1)^{n} \cdot \prod_{k=0}^{n-1} p(k)
$$

und $c \in \mathbb{R}$ darstellbar. 


\section{Beispiel 2.2.4}

$$
x(n+1)-(n+1) \cdot x(n)=0, \quad n \in \mathbb{N}_{0} .
$$

Es gilt für jedes $n \in \mathbb{N}_{0}$ :

$$
p(n)=(-1) \cdot(n+1)
$$

Dann ist

$$
\prod_{k=0}^{n-1} p(k)=\prod_{k=0}^{n-1}(-1) \cdot(k+1)=(-1)^{n} \cdot n ! .
$$

Nach Bemerkung 2.2.2 ist

$$
x_{1}(n)=(-1)^{n} \cdot \prod_{k=0}^{n-1} p(k)=(-1)^{n} \cdot(-1)^{n} \cdot n !=n !
$$

eine Lösung und nach Satz 2.2.3 ist

$$
x(n)=c \cdot n ! \quad \text { mit beliebigem } \quad c \in \mathbb{R}
$$

die allgemeine Lösung der Gleichung (2.2.4) auf $\mathbb{N}_{0}$.

\section{Folgerung 2.2.5 (Eindeutigkeit der Lösung des Anfangswertproblems)}

Gegeben sei die Funktion $p: \mathbb{N}_{0} \rightarrow \mathbb{R}$, wobei $p(n) \neq 0$ für alle $n \in \mathbb{N}_{0}$ ist, sowie $n_{0} \in \mathbb{N}_{0}$. Dann existiert für beliebiges $\mu_{0} \in \mathbb{R}$ genau eine Lösung des Anfangswertproblems

$$
\left\{\begin{aligned}
x(n+1)+p(n) \cdot x(n) & =0, \quad n \in \mathbb{N}_{0}, \\
x\left(n_{0}\right) & =\mu_{0} .
\end{aligned}\right.
$$

BEwEIS:

Nach Satz 2.2.3 ist die Funktion $x: \mathbb{N}_{0} \rightarrow \mathbb{R}$ mit

$$
x(n)=c \cdot(-1)^{n} \cdot \prod_{k=0}^{n-1} p(k)
$$

und beliebigem $c \in \mathbb{R}$ die allgemeine Lösung der Gleichung

$$
x(n+1)+p(n) \cdot x(n)=0 \quad \text { auf } \mathbb{N}_{0} .
$$


Mit dem Anfangswert $\mu_{0} \in \mathbb{R}$ können wir die Konstante eindeutig auswählen:

$$
x\left(n_{0}\right)=\mu_{0} \quad \Leftrightarrow \quad c \cdot(-1)^{n_{0}} \cdot \prod_{k=0}^{n_{0}-1} p(k)=\mu_{0} \quad \Leftrightarrow \quad c=\frac{\mu_{0}}{(-1)^{n_{0}} \cdot \prod_{k=0}^{n_{0}-1} p(k)} .
$$

Also ist die Funktion $x: \mathbb{N}_{0} \rightarrow \mathbb{R}$ mit

$$
x(n):=\mu_{0} \cdot(-1)^{n-n_{0}} \cdot \frac{\prod_{k=0}^{n-1} p(k)}{\prod_{k=0}^{n_{0}-1} p(k)}
$$

die eindeutig bestimmte Lösung des Anfangswertproblems (2.2.5).

Beachte: Es gilt

$$
x(n)=\left\{\begin{aligned}
\mu_{0} \cdot(-1)^{n-n_{0}} \cdot \prod_{k=n_{0}}^{n-1} p(k), & \text { falls } \quad n \geqslant n_{0}+1, \\
\mu_{0}, & \text { falls } \quad n=n_{0}, \\
\mu_{0} \cdot(-1)^{n-n_{0}} \cdot \frac{1}{\prod_{k=n}^{n_{0}-1} p(k)}, & \text { falls } \quad 0 \leqslant n \leqslant n_{0}-1 .
\end{aligned}\right.
$$

\section{Beispiel 2.2.6 (Anfangswertproblem)}

$$
\left\{\begin{aligned}
x(n+1)+\left(1+\frac{1}{n+1}\right) \cdot x(n) & =0, \quad n \in \mathbb{N}_{0}, \\
x(5) & =12 .
\end{aligned}\right.
$$

1) Es gilt für alle $n \in \mathbb{N}_{0}$ :

$$
p(n)=1+\frac{1}{n+1}=\frac{n+2}{n+1} .
$$

Dann ist

$$
\prod_{k=0}^{n-1} p(k)=\prod_{k=0}^{n-1} \frac{k+2}{k+1}=\frac{2}{1} \cdot \frac{3}{2} \cdots \frac{n+1}{n}=n+1 .
$$


Nach Bemerkung 2.2.2 ist

$$
x_{1}(n)=(-1)^{n} \cdot(n+1)
$$

eine Lösung und nach Satz 2.2.3 ist

$$
x(n)=c \cdot(-1)^{n} \cdot(n+1)
$$

mit beliebigem $c \in \mathbb{R}$ die allgemeine Lösung der Gleichung

$$
x(n+1)+\left(1+\frac{1}{n+1}\right) \cdot x(n)=0 \quad \text { auf } \quad \mathbb{N}_{0} .
$$

2) Weiter gilt

$$
x(5)=12 \quad \Leftrightarrow \quad c \cdot(-1)^{5} \cdot 6=12 \quad \Leftrightarrow \quad c=-2
$$

Also ist die Funktion $x: \mathbb{N}_{0} \rightarrow \mathbb{R}$ mit

$$
x(n)=(-2) \cdot(-1)^{n} \cdot(n+1)=2 \cdot(-1)^{n+1} \cdot(n+1)
$$

die Lösung des Anfangswertproblems (2.2.6).

\section{Bemerkung 2.2.7}

Lineare homogene Differenzengleichung erster Ordnung mit konstantem Koeffizienten

Sei $p \in \mathbb{R} \backslash\{0\}$. Gesucht ist eine Funktion $x: \mathbb{N}_{0} \rightarrow \mathbb{R}$, sodass für alle $n \in \mathbb{N}_{0}$ gilt:

$$
x(n+1)+p \cdot x(n)=0 .
$$

Für alle $n \in \mathbb{N}_{0}$ ist hier $p(n)=p$ und

$$
\prod_{k=0}^{n-1} p(k)=\prod_{k=0}^{n-1} p=p^{n}
$$

Nach Bemerkung 2.2.2 ist die Funktion $x_{1}: \mathbb{N}_{0} \rightarrow \mathbb{R}$ mit

$$
x_{1}(n):=(-1)^{n} \cdot \prod_{k=0}^{n-1} p(k)=(-1)^{n} \cdot p^{n}=(-p)^{n}
$$

eine Lösung und nach Satz 2.2.3 ist

$$
x(n)=c \cdot x_{1}(n)=c \cdot(-p)^{n}
$$

mit beliebigem $c \in \mathbb{R}$ die allgemeine Lösung der Gleichung (2.2.7) auf $\mathbb{N}_{0}$. 


\section{Beispiel 2.2.8}

Seien $b, q \in \mathbb{R}$. Die unendliche Folge $\left\langle b_{n}\right\rangle_{n \in \mathbb{N}_{0}}$ die rekursiv durch $b_{n}:=b_{n-1} \cdot q$ für $n \geqslant 1$ mit dem Anfangsglied $b_{0}:=b$ definiert ist, heißt geometrische Folge. Wir können diese als folgendes Anfangswertproblem auffassen:

$$
\left\{\begin{aligned}
x(n+1)-q \cdot x(n) & =0, \quad n \in \mathbb{N}_{0}, \\
x(0) & =b .
\end{aligned}\right.
$$

$\underline{\text { I. Fall } q \neq 0}$

1) Nach Bemerkung 2.2.7 ist $x(n)=c \cdot(-(-q))^{n}=c \cdot q^{n}$ mit beliebigem $c \in \mathbb{R}$ die allgemeine Lösung der Gleichung $x(n+1)-q \cdot x(n)=0$ auf $\mathbb{N}_{0}$.

2) Weiter gilt

$$
x(0)=b \quad \Leftrightarrow \quad c \cdot q^{0}=b \quad \Leftrightarrow \quad c=b .
$$

Also ist die Funktion $x: \mathbb{N}_{0} \rightarrow \mathbb{R}$ mit $x(n):=b \cdot q^{n}$ die eindeutige Lösung dieses Anfangswertproblems.

II. Fall $q=0$

Dann gilt

$$
\left\{\begin{aligned}
x(n+1) & =0, \quad n \in \mathbb{N}_{0}, \\
x(0) & =b .
\end{aligned}\right.
$$

Für alle $q \in \mathbb{R}$ gilt hierbei $q^{0}:=1$. Also ist die Funktion $x: \mathbb{N}_{0} \rightarrow \mathbb{R}$ mit $x(n):=b \cdot q^{n}$ auch in diesem Fall die eindeutige Lösung des Anfangswertproblems.

Bemerkung: Für die geometrische Folge $\left\langle b_{n}\right\rangle_{n \in \mathbb{N}_{0}}$ gilt: $\quad b_{n}=b \cdot q^{n}$.

\subsection{Lineare inhomogene Differenzengleichung erster Ordnung}

Satz 2.3.1

Gegeben seien die Funktionen $p, f: \mathbb{N}_{0} \rightarrow \mathbb{R}$, wobei $p(n) \neq 0$ für alle $n \in \mathbb{N}_{0}$. Dann lässt sich die Lösungsmenge $\mathbb{L}$ der inhomogenen Differenzengleichung erster Ordnung

$$
x(n+1)+p(n) \cdot x(n)=f(n)
$$


auf $\mathbb{N}_{0}$ schreiben als: $\mathbb{L}=x^{*}(n)+\mathbb{L}_{0}$, wobei $\mathbb{L}_{0}$ die Lösungsmenge der entsprechenden homogenen Differenzengleichung

$$
x(n+1)+p(n) \cdot x(n)=0
$$

und $x^{*}(n)$ eine partikuläre Lösung der inhomogenen Differenzengleichung ist.

BeweIs:

Wir haben bereits in Satz 2.2.3 gesehen, dass $\mathbb{L}_{0}$ nichtleer ist. Wir werden in Bemerkung 2.3.4 zeigen, dass auch $\mathbb{L} \neq \varnothing$, mit anderen Worten existiert eine partikuläre Lösung $x^{*}(n)$ von (2.3.1) und es gilt auf $\mathbb{N}_{0}$

$$
x^{*}(n+1)+p(n) \cdot x^{*}(n)=f(n) .
$$

1. Sei $x_{L}(n) \in \mathbb{L}$ eine beliebige Lösung von (2.3.1). Dann gilt

$$
x_{L}(n+1)+p(n) \cdot x_{L}(n)=f(n)
$$

auf $\mathbb{N}_{0}$ und somit

$$
x_{L}(n+1)-x^{*}(n+1)+p(n) \cdot\left(x_{L}(n)-x^{*}(n)\right)=0 .
$$

D.h., $\widetilde{x}(n)=x_{L}(n)-x^{*}(n)$ ist eine Lösung der homogenen Differenzengleichung

$$
x(n+1)+p(n) \cdot x(n)=0
$$

auf $\mathbb{N}_{0}$. Also gilt für jedes $x_{L}(n) \in \mathbb{L}: x_{L}(n)=x^{*}(n)+\widetilde{x}(n) \in x^{*}(n)+\mathbb{L}_{0}$ und $\mathbb{L} \subseteq x^{*}(n)+\mathbb{L}_{0}$.

2. Sei $\widetilde{x}(n) \in \mathbb{L}_{0}$ eine beliebige Lösung der homogenen Differenzengleichung. Dann gilt auf $\mathbb{N}_{0}$ :

$$
\widetilde{x}(n+1)+p(n) \cdot \widetilde{x}(n)=0
$$

Da $x^{*}(n)$ eine partikuläre Lösung der inhomogenen Differenzengleichung (2.3.1) ist, folgt:

$$
\widetilde{x}(n+1)+x^{*}(n+1)+p(n) \cdot\left(\widetilde{x}(n)+x^{*}(n)\right)=f(n) .
$$

D.h., $x(n)=\widetilde{x}(n)+x^{*}(n)$ ist eine Lösung der inhomogenen Differenzengleichung (2.3.1) auf $\mathbb{N}_{0}$. Also gilt für jedes $\widetilde{x}(n) \in \mathbb{L}_{0}: x^{*}(n)+\widetilde{x}(n) \in \mathbb{L}$ und $x^{*}(n)+\mathbb{L}_{0} \subseteq \mathbb{L}$.

Insgesamt haben wir $\mathbb{L}=x^{*}(n)+\mathbb{L}_{0}$ gezeigt. 


\section{Folgerung 2.3.2}

Wie wir schon wissen, ist

$$
x_{1}(n):=(-1)^{n} \cdot \prod_{k=0}^{n-1} p(k)
$$

immer eine Lösung der homogenen Differenzengleichung (2.3.2) auf $\mathbb{N}_{0}$. Lässt man in $c \cdot x_{1}(n)$ die Zahl c die Menge der reellen Zahlen durchlaufen, so erhält man alle Lösungen der Gleichung (2.3.2).

Also gilt für die Lösungsmenge $\mathbb{L}_{0}$ :

$$
\mathbb{L}_{0}=\left\{c \cdot x_{1}(n): c \in \mathbb{R}\right\},
$$

dabei heißt $c \cdot x_{1}(n)$ allgemeine Lösung der homogenen Differenzengleichung. Ist jetzt $x^{*}(n)$ eine partikuläre Lösung der inhomogenen Differenzengleichung (2.3.1), dann gilt nach Satz 2.3.1 für die Lösungsmenge $\mathbb{L}$ :

$$
\mathbb{L}=\left\{c \cdot x_{1}(n)+x^{*}(n): c \in \mathbb{R}\right\}
$$

Man sagt: Durch

$$
x(n)=c \cdot x_{1}(n)+x^{*}(n)
$$

ist die allgemeine Lösung der linearen inhomogenen Differenzengleichung erster Ordnung (2.3.1) gegeben.

Oder auch: Die allgemeine Lösung der linearen inhomogenen Differenzengleichung erster Ordnung hat die Form

$$
x(n)=\widetilde{x}(n)+x^{*}(n),
$$

wobei $\widetilde{x}(n)$ die allgemeine Lösung der entsprechenden linearen homogenen Differenzengleichung erster Ordnung und $x^{*}(n)$ eine partikuläre Lösung der inhomogenen Differenzengleichung ist. 


\section{Beispiel 2.3.3}

Seien $a, d \in \mathbb{R}$. Die unendliche Folge $\left\langle a_{n}\right\rangle_{n \in \mathbb{N}_{0}}$, die rekursiv durch

$$
a_{n}:=a_{n-1}+d
$$

für $n \geqslant 1$ mit dem Anfangsglied $a_{0}:=a$ definiert ist, heißt arithmetische Folge. Wir können diese als folgendes Anfangswertproblem auffassen:

$$
\left\{\begin{aligned}
x(n+1)-x(n) & =d, \quad n \in \mathbb{N}_{0}, \\
x(0) & =a .
\end{aligned}\right.
$$

1) Die entsprechende homogene Differenzengleichung lautet

$$
x(n+1)-x(n)=0 .
$$

Hier gilt $p=-1$ und nach Bemerkung 2.2.7 ist

$$
\widetilde{x}(n)=c \cdot(-(-1))^{n}=c
$$

mit beliebigem $c \in \mathbb{R}$ die allgemeine Lösung der Gleichung

$$
x(n+1)-x(n)=0 \quad \text { auf } \quad \mathbb{N}_{0} .
$$

2) Wie ist nun $x^{*}(n)$ zu wählen, falls $d \neq 0$ ?

Dazu betrachten wir den Ansatz $x^{*}(n):=A \cdot n$ mit $A \in \mathbb{R}$. Dann gilt für jedes $n \in \mathbb{N}_{0}$ :

$$
x^{*}(n+1)=A \cdot(n+1)
$$

und

$$
x^{*}(n+1)-x^{*}(n)=d \quad \Leftrightarrow \quad A \cdot(n+1)-A \cdot n=d \quad \Leftrightarrow \quad A=d .
$$

Dann ist $x^{*}(n)=d \cdot n$ eine partikuläre Lösung der inhomogenen Gleichung. Nach Satz 2.3.1 und Folgerung 2.3.2 ist

$$
x(n)=\widetilde{x}(n)+x^{*}(n)=c+d \cdot n
$$

mit $c \in \mathbb{R}$ die allgemeine Lösung dieser inhomogenen Gleichung auf $\mathbb{N}_{0}$. 
3) Weiter gilt

$$
x(0)=a \quad \Leftrightarrow \quad c=a .
$$

Also ist die Funktion $x: \mathbb{N}_{0} \rightarrow \mathbb{R}$ mit

$$
x(n):=a+d \cdot n
$$

die Lösung dieses Anfangswertproblems.

Bemerkung: Für die arithmetische Folge $\left\langle a_{n}\right\rangle_{n \in \mathbb{N}_{0}}$ gilt: $\quad a_{n}=a+d \cdot n$.

\section{Bemerkung 2.3.4 (Variation der Konstanten)}

Gegeben seien die Funktionen $p, f: \mathbb{N}_{0} \rightarrow \mathbb{R}$, wobei $p(n) \neq 0$ für alle $n \in \mathbb{N}_{0}$ ist. Nach Bemerkung 2.2.2 ist $x_{1}: \mathbb{N}_{0} \rightarrow \mathbb{R}$ mit

$$
x_{1}(n):=(-1)^{n} \cdot \prod_{k=0}^{n-1} p(k)
$$

eine Lösung der homogenen Differenzengleichung

$$
x(n+1)+p(n) \cdot x(n)=0
$$

auf $\mathbb{N}_{0}$. Eine partikuläre Lösung der inhomogenen Differenzengleichung

$$
x(n+1)+p(n) \cdot x(n)=f(n)
$$

findet man nach dem Verfahren der Variation der Konstanten:

Wir suchen eine partikuläre Lösung in der Form

$$
x^{*}(n)=c(n) \cdot x_{1}(n),
$$

wobei $c: \mathbb{N}_{0} \rightarrow \mathbb{R}$ eine unbekannte Funktion ist. Dann gilt:

$$
x^{*}(n+1)=c(n+1) \cdot x_{1}(n+1) .
$$

In die Differenzengleichung (2.3.1) eingesetzt ergibt das auf $\mathbb{N}_{0}$ :

$$
\begin{aligned}
& c(n+1) \cdot x_{1}(n+1)+p(n) \cdot c(n) \cdot x_{1}(n)=f(n) \\
& \Leftrightarrow \quad c(n+1) \cdot x_{1}(n+1)-c(n) \cdot x_{1}(n+1)+c(n) \cdot x_{1}(n+1) \\
& +p(n) \cdot c(n) \cdot x_{1}(n)=f(n) \\
& \Leftrightarrow \quad(c(n+1)-c(n)) \cdot x_{1}(n+1)+c(n) \cdot \underbrace{\left(x_{1}(n+1)+p(n) \cdot x_{1}(n)\right)}_{=0}=f(n) \\
& \Leftrightarrow \quad(c(n+1)-c(n)) \cdot x_{1}(n+1)=f(n),
\end{aligned}
$$


wobei wir ausgenutzt haben, dass die Funktion $x_{1}$ Lösung der homogenen Differenzengleichung ist. Da

$$
x_{1}(n+1)=(-1)^{n+1} \cdot \prod_{k=0}^{n} p(k) \neq 0
$$

auf $\mathbb{N}_{0}$ ist, erhalten wir

$$
c(n+1)-c(n)=\frac{f(n)}{x_{1}(n+1)} .
$$

Daraus folgt

$$
\begin{aligned}
c(n+1)-c(0) & =\sum_{k=0}^{n} \frac{f(k)}{x_{1}(k+1)} \\
\Leftrightarrow \quad c(n+1) & =\sum_{k=0}^{n} \frac{f(k)}{x_{1}(k+1)}+c(0)
\end{aligned}
$$

sowie

$$
c(n)=\sum_{k=0}^{n-1} \frac{f(k)}{x_{1}(k+1)}+c(0),
$$

wobei $c(0) \in \mathbb{R}$ beliebig gewählt werden kann.

Beispiel 2.3.5

$$
x(n+1)-\left(\frac{n+1}{n+2}\right)^{2} \cdot x(n)=\frac{2}{n+2}, \quad n \in \mathbb{N}_{0} .
$$

1) Die entsprechende homogene Differenzengleichung lautet

$$
x(n+1)-\left(\frac{n+1}{n+2}\right)^{2} \cdot x(n)=0 .
$$

Für alle $n \in \mathbb{N}_{0}$ gilt: $\quad p(n)=(-1) \cdot\left(\frac{n+1}{n+2}\right)^{2}$. Nach Bemerkung 2.2.2 ist

$$
\begin{aligned}
x_{1}(n) & =(-1)^{n} \cdot \prod_{k=0}^{n-1}(-1) \cdot\left(\frac{k+1}{k+2}\right)^{2} \\
& =(-1)^{n} \cdot(-1)^{n} \cdot\left(\frac{1}{2}\right)^{2} \cdot\left(\frac{2}{3}\right)^{2} \cdot \ldots \cdot\left(\frac{n}{n+1}\right)^{2} \\
& =\frac{1}{(n+1)^{2}}
\end{aligned}
$$


eine Lösung und nach Satz 2.2.3 ist

$$
\widetilde{x}(n)=\widetilde{c} \cdot \frac{1}{(n+1)^{2}}
$$

mit beliebigem $\widetilde{c} \in \mathbb{R}$ die allgemeine Lösung der entsprechenden homogenen Differenzengleichung (2.3.5h) auf $\mathbb{N}_{0}$.

2) Wir suchen nun $x^{*}(n)$ mit dem Verfahren der Variation der Konstanten. Nach Bemerkung 2.3.4 erhalten wir auf $\mathbb{N}_{0}$

$$
\begin{aligned}
c(n)-c(0) & =\sum_{k=0}^{n-1} \frac{f(k)}{x_{1}(k+1)}=\sum_{k=0}^{n-1} \frac{2 \cdot(k+2)^{2}}{k+2}=2 \cdot \sum_{k=0}^{n-1}(k+2) \\
& =2 \cdot \sum_{k=0}^{n-1} k+4 \cdot \sum_{k=0}^{n-1} 1=2 \cdot \frac{(n-1) \cdot n}{2}+4 \cdot n \\
& =n^{2}+3 n .
\end{aligned}
$$

Also ist

$$
c(n)=n^{2}+3 n+c(0) .
$$

Wir nehmen (z.B.) $c(0)=2$ und bekommen folgende partikuläre Lösung

$$
\begin{aligned}
x^{*}(n) & =c(n) \cdot x_{1}(n)=\frac{n^{2}+3 n+2}{(n+1)^{2}}=\frac{(n+1) \cdot(n+2)}{(n+1)^{2}} \\
& =\frac{n+2}{n+1}
\end{aligned}
$$

Die allgemeine Lösung der inhomogenen Differenzengleichung (2.3.5) auf $\mathbb{N}_{0}$ ist somit

$$
x(n)=\widetilde{x}(n)+x^{*}(n)=\widetilde{c} \cdot \frac{1}{(n+1)^{2}}+\frac{n+2}{n+1} \quad \text { mit } \quad \widetilde{c} \in \mathbb{R} .
$$

\section{Bemerkung 2.3.6 (Konstruktion der Lösung des Anfangswertproblems)}

$$
\left\{\begin{aligned}
x(n+1)+p(n) \cdot x(n) & =f(n), \quad n \in \mathbb{N}_{0}, \\
x\left(n_{0}\right) & =\mu_{0} .
\end{aligned}\right.
$$


BeweIs:

Nach Satz 2.1.2 existiert genau eine Lösung des Anfangswertproblems (2.3.6). Diese kann wie folgt konstruiert werden: Nach Satz 2.2.3 ist

$$
\widetilde{x}(n)=\widetilde{c} \cdot x_{1}(n) \quad \text { mit beliebigem } \quad \widetilde{c} \in \mathbb{R}
$$

die allgemeine Lösung der entsprechenden homogenen Gleichung auf $\mathbb{N}_{0}$, wobei

$$
x_{1}(n):=(-1)^{n} \cdot \prod_{k=0}^{n-1} p(k) \neq 0 \quad \text { für alle } \quad n \in \mathbb{N}_{0} .
$$

Nach Bemerkung 2.3.4 betrachten wir die Funktion $c: \mathbb{N}_{0} \rightarrow \mathbb{R}$ mit

$$
c(n):=\sum_{k=0}^{n-1} \frac{f(k)}{x_{1}(k+1)}-\sum_{k=0}^{n_{0}-1} \frac{f(k)}{x_{1}(k+1)},
$$

wobei wir hierbei

$$
c(0):=\sum_{k=0}^{n_{0}-1} \frac{f(k)}{x_{1}(k+1)},
$$

gewählt haben. Es ist also

$$
c(n)=\left\{\begin{array}{cl}
\sum_{k=n_{0}}^{n-1} \frac{f(k)}{x_{1}(k+1)}, & \text { falls } \quad n \geqslant n_{0}+1, \\
0, & \text { falls } \quad n=n_{0}, \\
-\sum_{k=n}^{n_{0}-1} \frac{f(k)}{x_{1}(k+1)}, & \text { falls } \quad 0 \leqslant n \leqslant n_{0}-1 .
\end{array}\right.
$$

Die Funktion $x^{*}: \mathbb{N}_{0} \rightarrow \mathbb{R}$ mit

$$
x^{*}(n):=c(n) \cdot x_{1}(n)
$$

ist eine partikuläre Lösung und

$$
x(n)=\widetilde{x}(n)+x^{*}(n)=(\widetilde{c}+c(n)) \cdot x_{1}(n)
$$

die allgemeine Lösung der inhomogenen Differenzengleichung

$$
x(n+1)+p(n) \cdot x(n)=f(n) \quad \text { auf } \mathbb{N}_{0} .
$$


Mit dem Anfangswert $\mu_{0} \in \mathbb{R}$ können wir nun die Konstante $\widetilde{c}$ bereits eindeutig bestimmen:

$$
x\left(n_{0}\right)=\mu_{0} \quad \Leftrightarrow \quad(\widetilde{c}+\underbrace{c\left(n_{0}\right)}_{=0}) \cdot x_{1}\left(n_{0}\right)=\mu_{0} \underset{x_{1}\left(n_{0}\right) \neq 0}{\Longleftrightarrow} \widetilde{c}=\frac{\mu_{0}}{x_{1}\left(n_{0}\right)} .
$$

Also ist die Funktion $x: \mathbb{N}_{0} \rightarrow \mathbb{R}$ mit

$$
x(n):=\left(\frac{\mu_{0}}{x_{1}\left(n_{0}\right)}+c(n)\right) \cdot x_{1}(n)
$$

die eindeutig bestimmte Lösung des Anfangswertproblems (2.3.6).

\section{Satz 2.3.7 (Superpositionsprinzip)}

Gegeben seien die Funktionen $p, f_{1}, f_{2}: \mathbb{N}_{0} \rightarrow \mathbb{R}$, wobei $p(n) \neq 0$ für alle $n \in \mathbb{N}_{0}$ ist. Seien weiter $x_{1}^{*}(n)$ eine partikuläre Lösung von

$$
x(n+1)+p(n) \cdot x(n)=f_{1}(n)
$$

und $x_{2}^{*}(n)$ eine partikuläre Lösung von

$$
x(n+1)+p(n) \cdot x(n)=f_{2}(n) .
$$

Dann ist

$$
x^{*}(n):=x_{1}^{*}(n)+x_{2}^{*}(n)
$$

eine partikuläre Lösung von

$$
x(n+1)+p(n) \cdot x(n)=f_{1}(n)+f_{2}(n) .
$$

BEwEIS:

Einsetzen von $x^{*}(n)$ in (2.3.8) liefert die Behauptung, weil $x_{1}^{*}(n)$ und $x_{2}^{*}(n)$ partikuläre Lösungen der entsprechenden Gleichungen sind.

In der Tat gilt für jedes $n \in \mathbb{N}_{0}$ :

$$
\begin{gathered}
x^{*}(n+1)+p(n) \cdot x^{*}(n)=\left(x_{1}^{*}(n+1)+x_{2}^{*}(n+1)\right) \\
+p(n) \cdot\left(x_{1}^{*}(n)+x_{2}^{*}(n)\right) \\
=\left(x_{1}^{*}(n+1)+p(n) \cdot x_{1}^{*}(n)\right) \\
\quad+\left(x_{2}^{*}(n+1)+p(n) \cdot x_{2}^{*}(n)\right) \\
\underset{(2.3 .7 \mathrm{a})}{(2.3 .7 \mathrm{~b})} f_{1}(n)+f_{2}(n) .
\end{gathered}
$$




\subsection{Lineare inhomogene Differenzengleichung erster Ordnung mit konstantem Koeffizien- ten}

\section{Bemerkung 2.4.1}

Gegeben seien eine Funktion $f: \mathbb{N}_{0} \rightarrow \mathbb{R}$ und eine reelle Zahl $p \in \mathbb{R} \backslash\{0\}$. Gesucht ist eine Funktion $x: \mathbb{N}_{0} \rightarrow \mathbb{R}$, sodass für alle $n \in \mathbb{N}_{0}$ gilt:

$$
x(n+1)+p \cdot x(n)=f(n) .
$$

Nach Folgerung 2.3.2 und Bemerkung 2.2.7 hat die allgemeine Lösung der linearen inhomogenen Differenzengleichung (2.4.1) die Form

$$
x(n)=\widetilde{x}(n)+x^{*}(n),
$$

wobei

$$
\widetilde{x}(n)=c \cdot(-p)^{n} \quad \text { mit } \quad c \in \mathbb{R}
$$

die allgemeine Lösung der entsprechenden linearen homogenen Differenzengleichung

$$
x(n+1)+p \cdot x(n)=0
$$

und $x^{*}(n)$ eine partikuläre Lösung der inhomogenen Differenzengleichung ist. In Bemerkung 2.3.4 haben wir $x^{*}(n)$ mit dem Verfahren der Variation der Konstanten gesucht. Wir betrachten nun die Form partikulärer Lösungen $x^{*}(n)$ von (2.4.1) für einige Sonderfälle der rechten Seite $f: \mathbb{N}_{0} \rightarrow \mathbb{R}$.

i) $f(n):=\gamma^{n} \cdot P_{m}(n)$, wobei $\gamma \in \mathbb{R} \backslash\{0\}$ und $P_{m}(n)$ ein Polynom vom Grad $m \in \mathbb{N}_{0}$ ist.

a) $\underline{y \neq-p}$. Wir suchen eine partikuläre Lösung in der Form

$$
x^{*}(n)=\gamma^{n} \cdot Q_{m}(n),
$$

wobei $Q_{m}(n)$ ein Polynom vom Grad $m$ mit unbekannten Koeffizienten ist.

Beispiel 2.4.2: $\quad x(n+1)+3 x(n)=n^{2}-4 n$,

Beispiel 2.4.3: $\quad x(n+1)-2 x(n)=4^{n} \cdot(n+1)$. 
b) $\underline{\gamma=-p}$. Wir suchen eine partikuläre Lösung in der Form

$$
x^{*}(n)=n \cdot \gamma^{n} \cdot Q_{m}(n),
$$

wobei $Q_{m}(n)$ ein Polynom vom Grad m mit unbekannten Koeffizienten ist. Beispiel 2.4.4: $\quad x(n+1)-5 x(n)=5^{n} \cdot(n-1)$.

ii) $f(n):=\gamma^{n} \cdot\left(P_{m}(n) \cdot \cos (n \psi)+Q_{l}(n) \cdot \sin (n \psi)\right)$, wobei $\gamma \in \mathbb{R} \backslash\{0\}$ und $\psi \in \mathbb{R} \backslash\{\pi k\}$ mit $k \in \mathbb{Z}$. Dabei sind $P_{m}(n), Q_{l}(n)$ Polynome vom Grad $m, l \in \mathbb{N}_{0}$ oder genau ein Polynom ist das Nullpolynom.

Wir suchen eine partikuläre Lösung in der Form

$$
x^{*}(n)=\gamma^{n} \cdot\left(S_{N}(n) \cdot \cos (n \psi)+T_{N}(n) \cdot \sin (n \psi)\right),
$$

wobei $S_{N}(n), T_{N}(n)$ Polynome vom Grad nicht mehr als $N:=\max \{l, m\}$ mit unbekannten Koeffizienten sind.

Beispiel 2.4.5: $\quad x(n+1)+2 x(n)=\cos \left(\frac{\pi}{2} \cdot n\right)$,

Beispiel 2.4.6: $\quad x(n+1)-4 x(n)=2^{n} \cdot \sin \left(\frac{\pi}{2} \cdot n\right)$.

\section{Beispiel 2.4.2}

$$
x(n+1)+3 x(n)=n^{2}-4 n, \quad n \in \mathbb{N}_{0} .
$$

1) Die entsprechende homogene Differenzengleichung lautet

$$
x(n+1)+3 x(n)=0 .
$$

Hier gilt $p=3$ und nach Bemerkung 2.2.7 ist

$$
\widetilde{x}(n)=c \cdot(-3)^{n}
$$

mit beliebigem $c \in \mathbb{R}$ die allgemeine Lösung der entsprechenden homogenen Differenzengleichung (2.4.2h) auf $\mathbb{N}_{0}$.

2) Wir haben $\gamma=1 \neq-3=-p$, sowie $P_{2}(n)=n^{2}-4 n$ und suchen nach Bemerkung 2.4.1 eine partikuläre Lösung der inhomogenen Gleichung in der Form

$$
x^{*}(n)=A \cdot n^{2}+B \cdot n+D \quad \text { mit } A, B, D \in \mathbb{R} .
$$


Wir haben

$$
x^{*}(n+1)=A \cdot(n+1)^{2}+B \cdot(n+1)+D
$$

und ein Einsetzen in die inhomogene Gleichung (2.4.2) ergibt:

$$
\begin{aligned}
& A \cdot\left(n^{2}+2 n+1\right)+B \cdot(n+1)+D+3 \cdot\left(A \cdot n^{2}+B \cdot n+D\right)=n^{2}-4 n \\
& \Leftrightarrow \quad 4 A \cdot n^{2}+(2 A+b+3 B) \cdot n+(A+B+D+3 D)=n^{2}-4 n \\
& \Leftrightarrow \quad 4 A \cdot n^{2}+(2 A+4 B) \cdot n+(A+B+4 D)=n^{2}-4 n \text {. }
\end{aligned}
$$

Ein Koeffizientenvergleich liefert:

$$
\left\{\begin{array} { l } 
{ 4 A = 1 } \\
{ 2 A + 4 B = - 4 } \\
{ A + B + 4 D = 0 }
\end{array} \Leftrightarrow \left\{\begin{array}{l}
A=\frac{1}{4} \\
B=-\frac{9}{8} \\
D=\frac{7}{32}
\end{array}\right.\right.
$$

Dann ist

$$
x^{*}(n)=\frac{n^{2}}{4}-\frac{9}{8} \cdot n+\frac{7}{32}
$$

eine partikuläre Lösung der inhomogenen Gleichung (2.4.2). Nach Satz 2.3.1 und Folgerung 2.3.2 ist

$$
x(n)=\widetilde{x}(n)+x^{*}(n)=c \cdot(-3)^{n}+\left(\frac{n^{2}}{4}-\frac{9}{8} \cdot n+\frac{7}{32}\right)
$$

mit beliebigem $c \in \mathbb{R}$ die allgemeine Lösung der inhomogenen Gleichung (2.4.2) auf $\mathbb{N}_{0}$.

Beispiel 2.4.3

$$
x(n+1)-2 x(n)=4^{n} \cdot(n+1), \quad n \in \mathbb{N}_{0} .
$$

1) Die entsprechende homogene Differenzengleichung lautet

$$
x(n+1)-2 x(n)=0 .
$$

Hier gilt $p=-2$ und nach Bemerkung 2.2.7 ist

$$
\widetilde{x}(n)=c \cdot 2^{n}
$$

mit beliebigem $c \in \mathbb{R}$ die allgemeine Lösung der entsprechenden homogenen Differenzengleichung (2.4.3h) auf $\mathbb{N}_{0}$. 
2) Wir haben $\gamma=4 \neq 2=-p$, sowie $P_{1}(n)=n+1$ und suchen nach Bemerkung 2.4.1 eine partikuläre Lösung der inhomogenen Gleichung in der Form

$$
x^{*}(n)=4^{n} \cdot(A \cdot n+B) \quad \text { mit } A, B \in \mathbb{R} .
$$

Wir haben

$$
x^{*}(n+1)=4^{n+1} \cdot(A \cdot(n+1)+B)
$$

und ein Einsetzen in die inhomogene Gleichung (2.4.3) ergibt:

$$
\begin{aligned}
& & 4^{n+1} \cdot(A \cdot(n+1)+B)-2 \cdot 4^{n} \cdot(A \cdot n+B) & =4^{n} \cdot(n+1) \\
& \Longleftrightarrow & 4 \cdot(A \cdot(n+1)+B)-2 \cdot(A \cdot n+B) & =n+1 \\
4^{n} \neq 0 & \Longleftrightarrow & 2 A \cdot n+(4 A+2 B) & =n+1 .
\end{aligned}
$$

Ein Koeffizientenvergleich liefert:

$$
\left\{\begin{array} { l } 
{ 2 A = 1 } \\
{ 4 A + 2 B = 1 }
\end{array} \Leftrightarrow \left\{\begin{array}{l}
A=\frac{1}{2} \\
B=-\frac{1}{2}
\end{array}\right.\right.
$$

Dann ist

$$
x^{*}(n)=4^{n} \cdot\left(\frac{n}{2}-\frac{1}{2}\right)
$$

eine partikuläre Lösung der inhomogenen Gleichung (2.4.3). Nach Satz 2.3.1 und Folgerung 2.3.2 ist

$$
x(n)=\widetilde{x}(n)+x^{*}(n)=c \cdot 2^{n}+4^{n} \cdot\left(\frac{n}{2}-\frac{1}{2}\right)
$$

mit beliebigem $c \in \mathbb{R}$ die allgemeine Lösung der inhomogenen Gleichung (2.4.3) auf $\mathbb{N}_{0}$. 


\section{Beispiel 2.4.4}

$$
x(n+1)-5 x(n)=5^{n} \cdot(n-1), \quad n \in \mathbb{N}_{0} .
$$

1) Die entsprechende homogene Differenzengleichung lautet

$$
x(n+1)-5 x(n)=0 .
$$

Hier gilt $p=-5$ und nach Bemerkung 2.2.7 ist

$$
\widetilde{x}(n)=c \cdot 5^{n}
$$

mit beliebigem $c \in \mathbb{R}$ die allgemeine Lösung der entsprechenden homogenen Differenzengleichung (2.4.4h) auf $\mathbb{N}_{0}$.

2) Wir haben $\gamma=5=-p$, sowie $P_{1}(n)=n-1$ und suchen nach Bemerkung 2.4.1 eine partikuläre Lösung der inhomogenen Gleichung in der Form

$$
x^{*}(n)=n \cdot 5^{n} \cdot(A \cdot n+B)=5^{n} \cdot\left(A \cdot n^{2}+B \cdot n\right) \quad \text { mit } A, B \in \mathbb{R} .
$$

Wir haben

$$
x^{*}(n+1)=5^{n+1} \cdot\left(A \cdot(n+1)^{2}+B \cdot(n+1)\right)
$$

und ein Einsetzen in die inhomogene Gleichung (2.4.4) ergibt:

$$
\begin{aligned}
& 5^{n+1} \cdot\left(A \cdot\left(n^{2}+2 n+1\right)+B \cdot(n+1)\right) \\
& -5 \cdot 5^{n} \cdot\left(A \cdot n^{2}+B \cdot n\right)=5^{n} \cdot(n-1) \\
& \underset{5^{n} \neq 0}{\Longleftrightarrow} \quad 5 \cdot\left(A \cdot\left(n^{2}+2 n+1\right)+B \cdot(n+1)\right) \\
& -5 \cdot\left(A \cdot n^{2}+B \cdot n\right)=n-1 \\
& \Longleftrightarrow \quad 5 \cdot(2 A+B-B) \cdot n+5 \cdot(A+B)=n-1 \\
& \Longleftrightarrow \quad 10 A \cdot n+5 \cdot(A+B)=n-1 \text {. }
\end{aligned}
$$

Ein Koeffizientenvergleich liefert:

$$
\left\{\begin{array} { l } 
{ 1 0 A = 1 } \\
{ 5 A + 5 B = - 1 }
\end{array} \Leftrightarrow \left\{\begin{array}{l}
A=\frac{1}{10} \\
B=-\frac{3}{10}
\end{array}\right.\right.
$$


Dann ist

$$
x^{*}(n)=5^{n} \cdot\left(\frac{n^{2}}{10}-\frac{3}{10} \cdot n\right)=\frac{5^{n-1}}{2} \cdot\left(n^{2}-3 \cdot n\right)
$$

eine partikuläre Lösung der inhomogenen Gleichung (2.4.4). Nach Satz 2.3.1 und Folgerung 2.3.2 ist

$$
x(n)=\widetilde{x}(n)+x^{*}(n)=c \cdot 5^{n}+\frac{5^{n-1}}{2} \cdot\left(n^{2}-3 \cdot n\right)
$$

mit beliebigem $c \in \mathbb{R}$ die allgemeine Lösung der inhomogenen Gleichung (2.4.4) auf $\mathbb{N}_{0}$.

\section{Beispiel 2.4.5}

$$
x(n+1)+2 x(n)=\cos \left(\frac{\pi}{2} \cdot n\right), \quad n \in \mathbb{N}_{0} .
$$

1) Die entsprechende homogene Differenzengleichung lautet

$$
x(n+1)+2 x(n)=0 .
$$

Hier gilt $p=2$ und nach Bemerkung 2.2.7 ist

$$
\widetilde{x}(n)=c \cdot(-2)^{n}
$$

mit beliebigem $c \in \mathbb{R}$ die allgemeine Lösung der entsprechenden homogenen Differenzengleichung (2.4.5h) auf $\mathbb{N}_{0}$.

2) Wir haben $\gamma=1, \psi=\frac{\pi}{2}, P_{0}(n)=1$ und $Q(n) \equiv 0$ (Nullpolynom) und suchen nach Bemerkung 2.4.1 eine partikuläre Lösung der inhomogenen Gleichung in der Form

$$
x^{*}(n)=A \cdot \cos \left(\frac{\pi}{2} \cdot n\right)+B \cdot \sin \left(\frac{\pi}{2} \cdot n\right) \quad \text { mit } A, B \in \mathbb{R} .
$$

Wir haben

$$
\begin{aligned}
x^{*}(n+1)= & A \cdot \cos \left(\frac{\pi}{2} \cdot(n+1)\right)+B \cdot \sin \left(\frac{\pi}{2} \cdot(n+1)\right) \\
= & A \cdot\left(\cos \left(\frac{\pi}{2} \cdot n\right) \cdot \cos \left(\frac{\pi}{2}\right)-\sin \left(\frac{\pi}{2} \cdot n\right) \cdot \sin \left(\frac{\pi}{2}\right)\right) \\
& +B \cdot\left(\sin \left(\frac{\pi}{2} \cdot n\right) \cdot \cos \left(\frac{\pi}{2}\right)+\cos \left(\frac{\pi}{2} \cdot n\right) \cdot \sin \left(\frac{\pi}{2}\right)\right) \\
= & A \cdot\left(-\sin \left(\frac{\pi}{2} \cdot n\right)\right)+B \cdot \cos \left(\frac{\pi}{2} \cdot n\right)
\end{aligned}
$$


und ein Einsetzen in die inhomogene Gleichung (2.4.5) ergibt:

$$
\begin{gathered}
A \cdot\left(-\sin \left(\frac{\pi}{2} \cdot n\right)\right)+B \cdot \cos \left(\frac{\pi}{2} \cdot n\right) \\
+2 \cdot\left(A \cdot \cos \left(\frac{\pi}{2} \cdot n\right)+B \cdot \sin \left(\frac{\pi}{2} \cdot n\right)\right)=\cos \left(\frac{\pi}{2} \cdot n\right) \\
\Leftrightarrow \quad(2 A+B) \cdot \cos \left(\frac{\pi}{2} \cdot n\right)+(-A+2 B) \cdot \sin \left(\frac{\pi}{2} \cdot n\right)=\cos \left(\frac{\pi}{2} \cdot n\right) .
\end{gathered}
$$

Ein Koeffizientenvergleich liefert:

$$
\left\{\begin{array} { l } 
{ 2 A + B = 1 } \\
{ - A + 2 B = 0 }
\end{array} \Leftrightarrow \left\{\begin{array}{l}
A=\frac{2}{5} \\
B=\frac{1}{5}
\end{array}\right.\right.
$$

Dann ist

$$
x^{*}(n)=\frac{1}{5} \cdot\left(2 \cdot \cos \left(\frac{\pi}{2} \cdot n\right)+\sin \left(\frac{\pi}{2} \cdot n\right)\right)
$$

eine partikuläre Lösung der inhomogenen Gleichung (2.4.5). Nach Satz 2.3.1 und Folgerung 2.3.2 ist

$$
x(n)=\widetilde{x}(n)+x^{*}(n)=c \cdot(-2)^{n}+\frac{1}{5} \cdot\left(2 \cdot \cos \left(\frac{\pi}{2} \cdot n\right)+\sin \left(\frac{\pi}{2} \cdot n\right)\right)
$$

mit beliebigem $c \in \mathbb{R}$ die allgemeine Lösung der inhomogenen Gleichung (2.4.5) auf $\mathbb{N}_{0}$.

\section{Beispiel 2.4.6}

$$
x(n+1)-4 x(n)=2^{n} \cdot \sin \left(\frac{\pi}{2} \cdot n\right), \quad n \in \mathbb{N}_{0} .
$$

1) Die entsprechende homogene Differenzengleichung lautet

$$
x(n+1)-4 x(n)=0 .
$$

Hier gilt $p=-4$ und nach Bemerkung 2.2.7 ist

$$
\widetilde{x}(n)=c \cdot 4^{n}
$$

mit beliebigem $c \in \mathbb{R}$ die allgemeine Lösung der entsprechenden homogenen Differenzengleichung (2.4.6h) auf $\mathbb{N}_{0}$. 
2) Wir haben $\gamma=2, \psi=\frac{\pi}{2}, P(n) \equiv 0$ (Nullpolynom) und $Q_{0}(n)=1$ und suchen nach Bemerkung 2.4.1 eine partikuläre Lösung der inhomogenen Gleichung in der Form

$$
x^{*}(n)=2^{n} \cdot\left(A \cdot \cos \left(\frac{\pi}{2} \cdot n\right)+B \cdot \sin \left(\frac{\pi}{2} \cdot n\right)\right) \quad \text { mit } A, B \in \mathbb{R} .
$$

Wir haben

$$
\begin{aligned}
x^{*}(n+1) & =2^{n+1} \cdot\left(A \cdot \cos \left(\frac{\pi}{2} \cdot(n+1)\right)+B \cdot \sin \left(\frac{\pi}{2} \cdot(n+1)\right)\right) \\
& =2^{n+1} \cdot\left(A \cdot\left(-\sin \left(\frac{\pi}{2} \cdot n\right)\right)+B \cdot \cos \left(\frac{\pi}{2} \cdot n\right)\right)
\end{aligned}
$$

und ein Einsetzen in die inhomogene Gleichung (2.4.6) ergibt:

$$
\begin{gathered}
2^{n+1} \cdot\left(A \cdot\left(-\sin \left(\frac{\pi}{2} \cdot n\right)\right)+B \cdot \cos \left(\frac{\pi}{2} \cdot n\right)\right) \\
-4 \cdot 2^{n} \cdot\left(A \cdot \cos \left(\frac{\pi}{2} \cdot n\right)+B \cdot \sin \left(\frac{\pi}{2} \cdot n\right)\right)=2^{n} \cdot \sin \left(\frac{\pi}{2} \cdot n\right) \\
\overleftrightarrow{2^{n} \neq 0} \quad(2 B-4 A) \cdot \cos \left(\frac{\pi}{2} \cdot n\right)+(-2 A-4 B) \cdot \sin \left(\frac{\pi}{2} \cdot n\right)=\sin \left(\frac{\pi}{2} \cdot n\right) .
\end{gathered}
$$

Ein Koeffizientenvergleich liefert:

$$
\left\{\begin{array} { l } 
{ - 4 A + 2 B = 0 } \\
{ - 2 A - 4 B = 1 }
\end{array} \Leftrightarrow \left\{\begin{array}{l}
A=-\frac{1}{10} \\
B=-\frac{2}{10}
\end{array}\right.\right.
$$

Dann ist

$$
x^{*}(n)=-\frac{2^{n}}{10} \cdot\left(\cos \left(\frac{\pi}{2} \cdot n\right)+2 \cdot \sin \left(\frac{\pi}{2} \cdot n\right)\right)
$$

eine partikuläre Lösung der inhomogenen Gleichung (2.4.6). Nach Satz 2.3.1 und Folgerung 2.3.2 ist

$$
x(n)=\widetilde{x}(n)+x^{*}(n)=c \cdot 4^{n}-\frac{2^{n}}{10} \cdot\left(\cos \left(\frac{\pi}{2} \cdot n\right)+2 \cdot \sin \left(\frac{\pi}{2} \cdot n\right)\right)
$$

mit beliebigem $c \in \mathbb{R}$ die allgemeine Lösung der inhomogenen Gleichung (2.4.6) auf $\mathbb{N}_{0}$. 


\section{Beispiel 2.4.7 (Anfangswertproblem)}

$\left\{\begin{aligned} x(n+1)-\frac{1}{2} \cdot x(n) & =\left(\frac{1}{2}\right)^{n}+\frac{1}{2 \cdot\left(2^{n}+1\right) \cdot\left(2^{n+1}+1\right)}, \quad n \in \mathbb{N}_{0}, \\ x(0) & =2 .\end{aligned}\right.$

1) Die entsprechende homogene Differenzengleichung lautet

$$
x(n+1)-\frac{1}{2} \cdot x(n)=0 .
$$

Hier gilt $p=-\frac{1}{2}$ und nach Bemerkung 2.2.7 haben wir

$$
x_{1}(n)=\left(\frac{1}{2}\right)^{n}
$$

Ferner ist

$$
\widetilde{x}(n)=\widetilde{c} \cdot\left(\frac{1}{2}\right)^{n}
$$

mit beliebigem $\widetilde{c} \in \mathbb{R}$ die allgemeine Lösung der entsprechenden homogenen Differenzengleichung (2.4.7h) auf $\mathbb{N}_{0}$.

2) Wir suchen eine partikuläre Lösung der inhomogenen Gleichung

$$
x(n+1)-\frac{1}{2} \cdot x(n)=\left(\frac{1}{2}\right)^{n}
$$

nach Bemerkung 2.4.1 in der Form

$$
x_{1}^{*}(n)=n \cdot\left(\frac{1}{2}\right)^{n} \cdot A \quad \text { mit } A \in \mathbb{R} .
$$

Wir haben

$$
x_{1}^{*}(n+1)=(n+1) \cdot\left(\frac{1}{2}\right)^{n+1} \cdot A
$$

und ein Einsetzen in die inhomogene Gleichung (2.4.7a) ergibt:

$$
\begin{aligned}
& & (n+1) \cdot\left(\frac{1}{2}\right)^{n+1} \cdot A-\frac{1}{2} \cdot n \cdot\left(\frac{1}{2}\right)^{n} \cdot A & =\left(\frac{1}{2}\right)^{n} \\
& \Longleftrightarrow & (n+1) \cdot \frac{1}{2} \cdot A-\frac{1}{2} \cdot n \cdot A & =1 \\
\left(\frac{1}{2}\right)^{n} \neq 0 & \Longleftrightarrow & \frac{1}{2} \cdot A & =1 \\
\Longleftrightarrow & & A & =2 .
\end{aligned}
$$


Dann ist

$$
x_{1}^{*}(n)=n \cdot\left(\frac{1}{2}\right)^{n} \cdot 2=n \cdot\left(\frac{1}{2}\right)^{n-1}
$$

eine partikuläre Lösung der inhomogenen Gleichung (2.4.7a) auf $\mathbb{N}_{0}$.

3) Wir suchen eine partikuläre Lösung der inhomogenen Gleichung

$$
x(n+1)-\frac{1}{2} \cdot x(n)=\frac{1}{2 \cdot\left(2^{n}+1\right) \cdot\left(2^{n+1}+1\right)}
$$

nach dem Verfahren der Variation der Konstanten in der Form

$$
x_{2}^{*}(n)=c(n) \cdot x_{1}(n)
$$

wobei $c: \mathbb{N}_{0} \rightarrow \mathbb{R}$ eine noch unbekannte Funktion ist.

Nach Bemerkung 2.3.4 gilt für jedes $n \in \mathbb{N}_{0}$ :

$$
\begin{aligned}
c(n)-c(0) & =\sum_{k=0}^{n-1} \frac{1}{2 \cdot\left(2^{k}+1\right) \cdot\left(2^{k+1}+1\right)} \cdot 2^{k+1} \\
& =\sum_{k=0}^{n-1} \frac{2^{k}}{\left(2^{k}+1\right) \cdot\left(2^{k+1}+1\right)}=\sum_{k=0}^{n-1} \frac{2^{k}}{\left(2^{k}+1\right) \cdot\left(2 \cdot 2^{k}+1\right)} \\
& =\sum_{k=0}^{n-1}\left(\frac{1}{\left(2^{k}+1\right)}-\frac{1}{2 \cdot 2^{k}+1}\right)=\sum_{k=0}^{n-1}\left(\frac{1}{\left(2^{k}+1\right)}-\frac{1}{2^{k+1}+1}\right) \\
& =\frac{1}{2}-\frac{1}{2^{n}+1} .
\end{aligned}
$$

Also ist

$$
c(n)=\frac{1}{2}-\frac{1}{2^{n}+1}+c(0) .
$$

Wir wählen (z.B.) $c(0)=0$. Dann haben wir für jedes $n \in \mathbb{N}_{0}$ die Gestalt:

$$
c(n)=\frac{1}{2}-\frac{1}{2^{n}+1}=\frac{2^{n}}{\left(2^{n}+1\right)} .
$$

Folgt:

$$
x_{2}^{*}(n)=\frac{2^{n}}{2^{n}+1} \cdot\left(\frac{1}{2}\right)^{n}=\frac{1}{2^{n}+1}
$$

ist eine partikuläre Lösung der inhomogenen Gleichung (2.4.7b) auf $\mathbb{N}_{0}$. 
4) Nach dem Superpositionsprinzip (Satz 2.3.7) ist

$$
x^{*}(n)=x_{1}^{*}(n)+x_{2}^{*}(n)
$$

eine partikuläre und

$$
x(n)=\widetilde{x}(n)+x^{*}(n)=\widetilde{c} \cdot\left(\frac{1}{2}\right)^{n}+n \cdot\left(\frac{1}{2}\right)^{n-1}+\frac{1}{2^{n}+1}
$$

die allgemeine Lösung der inhomogenen Gleichung

$$
x(n+1)-\frac{1}{2} \cdot x(n)=\left(\frac{1}{2}\right)^{n}+\frac{1}{2 \cdot\left(2^{n}+1\right) \cdot\left(2^{n+1}+1\right)}
$$

auf $\mathbb{N}_{0}$.

5) Mit Hilfe des Anfangswertes $x(0)=2$ bekommen wir

$$
x(0)=2 \quad \Leftrightarrow \quad \widetilde{c} \cdot 1+0+\frac{1}{2}=2 \quad \Leftrightarrow \quad \widetilde{c}=\frac{3}{2} .
$$

Dann ist

$$
x(n)=\widetilde{x}(n)+x^{*}(n)=\frac{3}{2} \cdot\left(\frac{1}{2}\right)^{n}+n \cdot\left(\frac{1}{2}\right)^{n-1}+\frac{1}{2^{n}+1}
$$

die eindeutige Lösung des Anfangswertproblems (2.4.7).

\section{Beispiel 2.4.8}

Im Beispiel 2.2.8 haben wir die geometrische Folge $\left\langle b_{n}\right\rangle_{n \in \mathbb{N}_{0}}$, die rekursiv durch $b_{n}:=b_{n-1} \cdot q$ für $n \geqslant 1$ mit dem Anfangsglied $b_{0}:=b$ definiert ist, untersucht und gezeigt, dass $b_{n}=b \cdot q^{n}$ für alle $n \in \mathbb{N}_{0}$ gilt (für alle $q \in \mathbb{R}$ ist hierbei $q^{0}:=1$ ). Die summierten Glieder dieser Folge bilden eine neue Folge $\left\langle\sigma_{n}\right\rangle_{n \in \mathbb{N}_{0}}$, die rekursiv durch $\sigma_{n}:=\sigma_{n-1}+b_{n}$ für $n \geqslant 1$ mit dem Anfangsglied $\sigma_{0}:=b_{0}$ definiert ist. Wir können diese als folgendes Anfangswertproblem auffassen:

$$
\left\{\begin{aligned}
x(n+1)-x(n) & =b \cdot q^{n+1}, \quad n \in \mathbb{N}_{0}, \\
x(0) & =b .
\end{aligned}\right.
$$

1) Die entsprechende homogene Differenzengleichung lautet

$$
x(n+1)-x(n)=0 .
$$


Hier gilt $p=-1$ und nach Bemerkung 2.2.7 ist

$$
\widetilde{x}(n)=c
$$

mit beliebigem $c \in \mathbb{R}$ die allgemeine Lösung der entsprechenden homogenen Differenzengleichung (2.4.8h) auf $\mathbb{N}_{0}$.

2) Wir suchen eine partikuläre Lösung der inhomogenen Gleichung

$$
x(n+1)-x(n)=b \cdot q^{n+1} .
$$

I. Fall: $q \neq 1, \quad q \neq 0, \quad b \neq 0$

Nach Bemerkung 2.4.1 suchen wir $x^{*}(n)$ in der Form

$$
x^{*}(n)=A \cdot b \cdot q^{n+1} \quad \text { mit } A \in \mathbb{R} .
$$

Wir haben

$$
x^{*}(n+1)=A \cdot b \cdot q^{n+2} .
$$

und ein Einsetzen in die inhomogene Gleichung ergibt:

$$
\begin{aligned}
A \cdot b \cdot q^{n+2}-A \cdot b \cdot q^{n+1} & =b \cdot q^{n+1} \\
& \Leftrightarrow \cdot(q-1)=1 \quad \underset{q \neq 1}{\Longleftrightarrow} \quad A=\frac{1}{q-1}
\end{aligned}
$$

Dann ist

$$
x^{*}(n)=\frac{b \cdot q^{n+1}}{q-1}
$$

eine partikuläre und

$$
x(n)=\widetilde{x}(n)+x^{*}(n)=c+\frac{b \cdot q^{n+1}}{q-1}
$$

die allgemeine Lösung der inhomogenen Gleichung.

Mit Hilfe des Anfangswertes $x(0)=b$ bekommen wir

$$
x(0)=b \quad \Leftrightarrow \quad c+\frac{b \cdot q}{q-1}=b \quad \Leftrightarrow \quad c=-\frac{b}{q-1} .
$$

Dann ist

$$
x(n)=-\frac{b}{q-1}+\frac{b \cdot q^{n+1}}{q-1}=b \cdot \frac{q^{n+1}-1}{q-1}
$$

die eindeutige Lösung des Anfangswertproblems (2.4.8) in diesem Fall. 
II. Fall: $q=1, \quad b \neq 0$

Die inhomogene Gleichung lautet

$$
x(n+1)-x(n)=b .
$$

Nach Bemerkung 2.4.1 suchen wir $x^{*}(n)$ in der Form

$$
x^{*}(n)=A \cdot n \quad \text { mit } A \in \mathbb{R} .
$$

Wir haben

$$
x^{*}(n+1)=A \cdot(n+1) .
$$

und ein Einsetzen in die inhomogene Gleichung ergibt:

$$
A \cdot(n+1)-A \cdot n=b \quad \Leftrightarrow \quad A=b .
$$

Dann ist $x^{*}(n)=b \cdot n$ eine partikuläre und

$$
x(n)=\widetilde{x}(n)+x^{*}(n)=c+b \cdot n
$$

die allgemeine Lösung der inhomogenen Gleichung. Mit Hilfe des Anfangswertes $x(0)=b$ bekommen wir

$$
x(0)=b \quad \Leftrightarrow \quad c=b .
$$

Dann ist

$$
x(n)=b+b \cdot n=b \cdot(n+1)
$$

die eindeutige Lösung des Anfangswertproblems (2.4.8) in diesem Fall.

III. Fall: $q=0$ oder $b=0$

Wir bekommen das Anfangswertproblem

$$
\left\{\begin{aligned}
x(n+1)-x(n) & =0, \quad n \in \mathbb{N}_{0}, \\
x(0) & =b .
\end{aligned}\right.
$$

Nach 1) ist $x(n)=c$ mit $c \in \mathbb{R}$ die allgemeine Lösung der Differenzengleichung. Mit Hilfe des Anfangswertes $x(0)=b$ bekommen wir

$$
x(0)=b \quad \Leftrightarrow \quad c=b .
$$

Dann ist

$$
x(n)=b
$$

die eindeutige Lösung des Anfangswertproblems (2.4.8) in diesem Fall. 
Bemerkung: Insgesamt gilt für die summierten Glieder einer geometrischen Folge $\left(n \in \mathbb{N}_{0}\right)$ :

$$
\sigma_{n}=\left\{\begin{array}{cll}
b \cdot \frac{q^{n+1}-1}{q-1}, & \text { falls } & q \neq 1 \\
b \cdot(n+1), & \text { falls } & q=1 .
\end{array}\right.
$$

\section{Beispiel 2.4.9}

Im Beispiel 2.3.3 haben wir die arithmetische Folge $\left\langle a_{n}\right\rangle_{n \in \mathbb{N}_{0}}$, die rekursiv durch $a_{n}:=a_{n-1}+d$ für $n \geqslant 1$ mit dem Anfangsglied $a_{0}:=a$ definiert ist, untersucht und gezeigt, dass $a_{n}=a+d \cdot n$ für alle $n \in \mathbb{N}_{0}$ gilt. Die summierten Glieder dieser Folge bilden eine neue Folge $\left\langle s_{n}\right\rangle_{n \in \mathbb{N}_{0}}$, die rekursiv durch $s_{n}:=s_{n-1}+a_{n}$ für $n \geqslant 1$ mit dem Anfangsglied $s_{0}:=a_{0}$ definiert ist. Wir können diese als folgendes Anfangswertproblem auffassen:

$$
\left\{\begin{aligned}
x(n+1)-x(n) & =a+d \cdot(n+1), \quad n \in \mathbb{N}_{0}, \\
x(0) & =a .
\end{aligned}\right.
$$

1) Die entsprechende homogene Differenzengleichung lautet

$$
x(n+1)-x(n)=0 .
$$

Hier gilt $p=-1$ und nach Bemerkung 2.2.7 ist

$$
\widetilde{x}(n)=c
$$

mit beliebigem $c \in \mathbb{R}$ die allgemeine Lösung der entsprechenden homogenen Differenzengleichung (2.4.9h) auf $\mathbb{N}_{0}$.

2) Nach Bemerkung 2.4.1 suchen wir eine partikuläre Lösung der inhomogenen Gleichung

$$
x(n+1)-x(n)=a+d \cdot(n+1)
$$

in der Form

$$
x^{*}(n)=n \cdot(A \cdot n+B)=A \cdot n^{2}+B \cdot n \quad \text { mit } A, B \in \mathbb{R} .
$$

Wir haben

$$
x^{*}(n+1)=A \cdot(n+1)^{2}+B \cdot(n+1)
$$


und ein Einsetzen in die inhomogene Gleichung ergibt:

$$
\begin{aligned}
& \left(A \cdot(n+1)^{2}+B \cdot(n+1)\right)-\left(A \cdot n^{2}+B \cdot n\right)=a+d \cdot(n+1) \\
& \Leftrightarrow \quad 2 A \cdot n+(A+B)=a+d \cdot(n+1) \text {. }
\end{aligned}
$$

Ein Koeffizientenvergleich liefert:

$$
\left\{\begin{array} { l } 
{ 2 A = d } \\
{ A + B = a + d }
\end{array} \Leftrightarrow \left\{\begin{array}{l}
A=\frac{d}{2} \\
B=a+\frac{d}{2}
\end{array}\right.\right.
$$

Dann ist

$$
x^{*}(n)=\frac{d}{2} \cdot n^{2}+\left(a+\frac{d}{2}\right) \cdot n
$$

eine partikuläre Lösung der inhomogenen Gleichung. Nach Satz 2.3.1 und Folgerung 2.3.2 ist

$$
x(n)=\widetilde{x}(n)+x^{*}(n)=c+\frac{d}{2} \cdot n^{2}+\left(a+\frac{d}{2}\right) \cdot n
$$

mit beliebigem $c \in \mathbb{R}$ die allgemeine Lösung dieser inhomogenen Gleichung auf $\mathbb{N}_{0}$.

3) Mit Hilfe des Anfangswertes $x(0)=a$ bekommen wir

$$
x(0)=a \quad \Leftrightarrow \quad c=a .
$$

Dann ist

$$
x(n)=a+\frac{d}{2} \cdot n^{2}+\left(a+\frac{d}{2}\right) \cdot n=a \cdot(n+1)+\frac{d \cdot n \cdot(n+1)}{2}
$$

die eindeutige Lösung des Anfangswertproblems (2.4.9).

Bemerkung: Für die summierten Glieder einer arithmetischen Folge gilt:

$$
s_{n}=a \cdot(n+1)+\frac{d \cdot n \cdot(n+1)}{2}, \quad n \in \mathbb{N}_{0} .
$$




\section{Lineare Differentialgleichungen zweiter Ordnung}

Generalvoraussetzung: Im Folgenden bezeichnet $I \subseteq \mathbb{R}$ ein beliebiges Intervall, d.h., $I$ ist eine nichtleere zusammenhängende Teilmenge von $\mathbb{R}$, die ein nichtleeres Inneres hat. Gehört ein Randpunkt zum Intervall, so betrachten wir für die Differenzierbarkeit in diesem Randpunkt einen einseitigen Grenzwert.

\subsection{Anfangswertproblem für lineare Differenti- algleichungen zweiter Ordnung}

\section{Definition 3.1.1}

Gegeben seien die Funktionen $p, q, f: I \rightarrow \mathbb{R}$, die auf einem Intervall I definiert und stetig sind. Gesucht ist eine zwei mal differenzierbare Funktion $y: I \rightarrow \mathbb{R}$, sodass für alle $x \in I$ gilt:

$$
y^{\prime \prime}(x)+p(x) \cdot y^{\prime}(x)+q(x) \cdot y(x)=f(x)
$$

Gleichung (3.1.1) heißt lineare (gewöhnliche) Differentialgleichung zweiter Ordnung. Die Funktion $y: I \rightarrow \mathbb{R}$ heißt Lösung der Gleichung (3.1.1) auf dem Intervall I.

\section{Satz und Definition 3.1.2}

Seien die Funktionen $p, q, f: I \rightarrow \mathbb{R}$ auf einem Intervall I definiert und stetig, sowie $x_{0} \in I$. Dann existiert für beliebige $y_{0}, y_{0}^{\prime} \in \mathbb{R}$ auf I genau eine Lösung des 
Anfangswertproblems

$$
\left\{\begin{aligned}
y^{\prime \prime}(x)+p(x) \cdot y^{\prime}(x)+q(x) \cdot y(x) & =f(x), \\
y\left(x_{0}\right) & =y_{0}, \\
y^{\prime}\left(x_{0}\right) & =y_{0}^{\prime} .
\end{aligned}\right.
$$

Für einen Beweis verweisen wir auf Heuser [1, Satz 21.4].

\section{Bemerkung und Definition 3.1.3}

Ist $f(x)=0$ für alle $x \in I$, so heißt Gleichung (3.1.1) lineare homogene Differentialgleichung zweiter Ordnung, andernfalls heißt diese lineare inhomogene Differentialgleichung zweiter Ordnung.

\subsection{Lineare homogene Differentialgleichung zweiter Ordnung}

Wir untersuchen zuerst die homogene Gleichung

$$
y^{\prime \prime}(x)+p(x) \cdot y^{\prime}(x)+q(x) \cdot y(x)=0
$$

(dabei sind die Funktionen $p, q: I \rightarrow \mathbb{R}$ auf $I$ definiert und stetig).

\section{Satz 3.2.1}

Seien $y_{1}, y_{2}: I \rightarrow \mathbb{R}$ zwei Lösungen von (3.2.1), dann ist $y(x):=c_{1} \cdot y_{1}(x)+$ $c_{2} \cdot y_{2}(x)$ ebenfalls eine Lösung von (3.2.1) auf $I$, wobei $c_{1}, c_{2} \in \mathbb{R}$ zwei beliebige Konstanten sind.

BeweIs:

Da $y_{1}$ und $y_{2}$ zwei Lösungen von (3.2.1) sind, gilt für alle $x \in I$ :

$$
y_{1}^{\prime \prime}(x)+p(x) \cdot y_{1}^{\prime}(x)+q(x) \cdot y_{1}(x)=0
$$

und

$$
y_{2}^{\prime \prime}(x)+p(x) \cdot y_{2}^{\prime}(x)+q(x) \cdot y_{2}(x)=0 .
$$


Dann folgt für $y(x):=c_{1} \cdot y_{1}(x)+c_{2} \cdot y_{2}(x)$, mit beliebigen Konstanten $c_{1}, c_{2} \in \mathbb{R}$ :

$$
\begin{aligned}
& y^{\prime \prime}(x)+p(x) \cdot y^{\prime}(x)+q(x) \cdot y(x) \\
& =\left[c_{1} \cdot y_{1}(x)+c_{2} \cdot y_{2}(x)\right]^{\prime \prime} \\
& +p(x) \cdot\left[c_{1} \cdot y_{1}(x)+c_{2} \cdot y_{2}(x)\right]^{\prime} \\
& +q(x) \cdot\left[c_{1} \cdot y_{1}(x)+c_{2} \cdot y_{2}(x)\right] \\
& =c_{1} \cdot\left[y_{1}^{\prime \prime}(x)+p(x) \cdot y_{1}^{\prime}(x)+q(x) \cdot y_{1}(x)\right] \\
& +c_{2} \cdot\left[y_{2}^{\prime \prime}(x)+p(x) \cdot y_{2}^{\prime}(x)+q(x) \cdot y_{2}(x)\right] \\
& \underset{(3.2 .2 \mathrm{~b})}{\stackrel{(3.2 .2 \mathrm{a})}{(.2}} 0 \quad \text { für alle } x \in I \text {. }
\end{aligned}
$$

Nach Definition 3.1.1 ist also

$$
y(x)=c_{1} \cdot y_{1}(x)+c_{2} \cdot y_{2}(x)
$$

eine Lösung von (3.2.1) auf $I$, wobei $c_{1}, c_{2} \in \mathbb{R}$ beliebige Konstanten sind.

\section{Definition 3.2.2 (Komplexwertige Funktion der reellen Variablen)}

Eine Abbildung $g: I \rightarrow \mathbb{C}$ heißt komplexwertige Funktion der reellen Variablen.

\section{Bemerkung und Definition 3.2.3}

i) Jede komplexwertige Funktion $g: I \rightarrow \mathbb{C}$ der reellen Variablen lässt sich in der Form $g=u+\mathrm{i} \cdot v$ schreiben, wobei $u, v: I \rightarrow \mathbb{R}$ zwei reellwertige Funktionen sind.

ii) Seien die Funktionen $u, v: I \rightarrow \mathbb{R}$ auf einem Intervall I definiert und stetig und an der Stelle $x_{0} \in I$ differenzierbar. Dann heißt die komplexwertige Funktion $g:=u+\mathrm{i} \cdot v$ an der Stelle $x_{0}$ differenzierbar und wir setzten

$$
g^{\prime}\left(x_{0}\right):=u^{\prime}\left(x_{0}\right)+\mathrm{i} \cdot v^{\prime}\left(x_{0}\right)
$$

iii) Seien die Funktionen $u, v: I \rightarrow \mathbb{R}$ auf einem Intervall I definiert und stetig, so besitzen sie dort ihre Stammfunktionen $U$ bzw. V. Dann ist die Funktion $G: I \rightarrow \mathbb{C}$ mit $G(x):=U(x)+\mathrm{i} \cdot V(x)$ eine Stammfunktion der Funktion $g=u+\mathrm{i} \cdot v$ auf $I$. 
In der Tat gilt nach ii) für alle $x \in I$ :

$$
(G(x))^{\prime}=(U(x)+\mathrm{i} \cdot V(x))^{\prime}=U^{\prime}(x)+\mathrm{i} \cdot V^{\prime}(x)=u(x)+\mathrm{i} \cdot v(x) .
$$

Ferner setzen wir

$$
\int g(x) \mathrm{d} x:=\int u(x) \mathrm{d} x+\mathrm{i} \cdot \int v(x) \mathrm{d} x
$$

auf I und

$$
\int_{c}^{d} g(x) \mathrm{d} x:=\int_{c}^{d} u(x) \mathrm{d} x+\mathrm{i} \cdot \int_{c}^{d} v(x) \mathrm{d} x,
$$

wobei $[c ; d] \subset I$ ein beliebiges beschränktes abgeschlossenes Intervall ist.

iv) Gegeben seien die Funktionen $p, q, f: I \rightarrow \mathbb{R}$, die auf einem Intervall $I$ definiert und stetig sind. Gesucht ist eine zwei mal differenzierbare Funktion $y: I \rightarrow \mathbb{C}$, sodass für alle $x \in I$ gilt:

$$
y^{\prime \prime}(x)+p(x) \cdot y^{\prime}(x)+q(x) \cdot y(x)=f(x) .
$$

Die Funktion y $I \rightarrow \mathbb{C}$ heißt (komplexwertige) Lösung dieser Gleichung.

\section{Beispiel 3.2.4}

Seien $\alpha, \beta \in \mathbb{R}$ und $\lambda:=(\alpha+\mathrm{i} \cdot \beta) \in \mathbb{C}$. Dann ist die Funktion $g: \mathbb{R} \rightarrow \mathbb{C}$ mit $g(x):=\mathrm{e}^{\lambda x}$ auf $\mathbb{R}$ wohldefiniert und es gilt für alle $x \in \mathbb{R}$ :

$$
\begin{aligned}
\mathrm{e}^{\lambda x} & =\mathrm{e}^{(\alpha+\mathrm{i} \cdot \beta) x}=\mathrm{e}^{\alpha x+\mathrm{i} \cdot \beta x}=\mathrm{e}^{\alpha x} \cdot \mathrm{e}^{\mathrm{i} \cdot \beta x} \stackrel{(*)}{=} \mathrm{e}^{\alpha x} \cdot(\cos (\beta x)+\mathrm{i} \cdot \sin (\beta x)) \\
& =\mathrm{e}^{\alpha x} \cdot \cos (\beta x)+\mathrm{i} \cdot \mathrm{e}^{\alpha x} \cdot \sin (\beta x),
\end{aligned}
$$

wobei wir in $(*)$ die EuLER'sche Formel ${ }^{3}$ benutzt haben:

\section{EULER'sche Formel}

$$
\mathrm{e}^{\mathrm{i} \cdot \xi}=\cos (\xi)+\mathrm{i} \cdot \sin (\xi) \quad \text { für alle } \xi \in \mathbb{R} .
$$

${ }^{3}$ Leonhard Euler, 1707-1783 
i) Nach Definition 3.2.3 ist die komplexwertige Funktion $g$ auf $\mathbb{R}$ differenzierbar und für jedes $x \in \mathbb{R}$ gilt:

$$
\begin{aligned}
\left(\mathrm{e}^{\lambda x}\right)^{\prime}= & \left(\mathrm{e}^{\alpha x} \cdot \cos (\beta x)+\mathrm{i} \cdot \mathrm{e}^{\alpha x} \cdot \sin (\beta x)\right)^{\prime} \\
= & \left(\mathrm{e}^{\alpha x} \cdot \cos (\beta x)\right)^{\prime}+\mathrm{i} \cdot\left(\mathrm{e}^{\alpha x} \cdot \sin (\beta x)\right)^{\prime} \\
= & \mathrm{e}^{\alpha x} \cdot \alpha \cdot \cos (\beta x)+\mathrm{e}^{\alpha x} \cdot(-\beta) \cdot \sin (\beta x) \\
& \quad+\mathrm{i} \cdot\left(\mathrm{e}^{\alpha x} \cdot \alpha \cdot \sin (\beta x)+\mathrm{e}^{\alpha x} \cdot \beta \cdot \cos (\beta x)\right) \\
\mathrm{i}^{2}=-1 & \alpha \cdot \mathrm{e}^{\alpha x} \cdot(\cos (\beta x)+\mathrm{i} \cdot \sin (\beta x)) \\
& \quad+\mathrm{i} \cdot \beta \cdot \mathrm{e}^{\alpha x} \cdot(\cos (\beta x)+\mathrm{i} \cdot \sin (\beta x)) \\
= & \alpha \cdot \mathrm{e}^{\lambda x}+\mathrm{i} \cdot \beta \cdot \mathrm{e}^{\lambda x}=\lambda \cdot \mathrm{e}^{\lambda x} .
\end{aligned}
$$

Also gilt für jedes $x \in \mathbb{R}$ :

$$
\left(\mathrm{e}^{\lambda x}\right)^{\prime}=\lambda \cdot \mathrm{e}^{\lambda x}, \quad \text { wobei } \lambda \in \mathbb{C} \text { ist. }
$$

ii) Gilt außerdem $\alpha^{2}+\beta^{2} \neq 0$, d.h., $\lambda=(\alpha+\mathrm{i} \cdot \beta) \in \mathbb{C} \backslash\{0\}$, so besitzt die komplexwertige Funktion $g$ eine Stammfunktion auf $\mathbb{R}$. Nach Definition 3.2.3 erhalten wir mit beliebigen $C_{1}, C_{2} \in \mathbb{R}$ :

$$
\begin{aligned}
\int \mathrm{e}^{\lambda x} \mathrm{~d} x= & \int \mathrm{e}^{\alpha x} \cdot \cos (\beta x) \mathrm{d} x+\mathrm{i} \cdot \int \mathrm{e}^{\alpha x} \cdot \sin (\beta x) \mathrm{d} x \\
\stackrel{(* *)}{=}\left[\frac{\mathrm{e}^{\alpha x}}{\alpha^{2}+\beta^{2}} \cdot(\alpha \cdot \cos (\beta x)+\beta \cdot \sin (\beta x))+C_{1}\right] & \\
& +\mathrm{i} \cdot\left[\frac{\mathrm{e}^{\alpha x}}{\alpha^{2}+\beta^{2}} \cdot(\alpha \cdot \sin (\beta x)-\beta \cdot \cos (\beta x))+C_{2}\right] \\
\stackrel{\mathrm{i}^{2}=-1}{=} \quad \frac{\alpha \cdot \mathrm{e}^{\alpha x}}{\alpha^{2}+\beta^{2}} \cdot(\cos (\beta x)+\mathrm{i} \cdot \sin (\beta x)) & \\
& -\mathrm{i} \cdot \frac{\beta \cdot \mathrm{e}^{\alpha x}}{\alpha^{2}+\beta^{2}} \cdot(\cos (\beta x)+\mathrm{i} \cdot \sin (\beta x))+\underbrace{\left(C_{1}+\mathrm{i} \cdot C_{2}\right)}_{=: C} \\
= & \frac{\mathrm{e}^{\alpha x} \cdot(\cos (\beta x)+\mathrm{i} \cdot \sin (\beta x)) \cdot(\alpha-\mathrm{i} \cdot \beta)}{\alpha^{2}+\beta^{2}}+C \quad \text { mit } C \in \mathbb{C} . \\
= & \frac{\mathrm{e}^{\lambda x} \cdot(\alpha-\mathrm{i} \cdot \beta)}{(\alpha+\mathrm{i} \cdot \beta) \cdot(\alpha-\mathrm{i} \cdot \beta)}+C=\frac{\mathrm{e}^{\lambda x}}{\lambda}+C \quad
\end{aligned}
$$


D.h., auf $\mathbb{R}$ gilt

$$
\int \mathrm{e}^{\lambda x} \mathrm{~d} x=\frac{1}{\lambda} \cdot \mathrm{e}^{\lambda x}+C \quad \text { mit } \lambda \in \mathbb{C} \backslash\{0\} \text { und } C \in \mathbb{C} \text {. }
$$

\section{Übung}

Weisen Sie die Umformung $(* *)$ in der obigen Rechnung nach.

\section{Satz 3.2.5}

Ist $y(x):=u(x)+\mathrm{i} \cdot v(x)$ mit den reellen Funktionen $u, v: I \rightarrow \mathbb{R}$ eine komplexwertige Lösung von (3.2.1), dann lösen diese beiden Funktionen ebenfalls diese Differentialgleichung.

BeweIs:

Da die Funktion $y=u+\mathrm{i} \cdot v$ eine komplexwertige Lösung von (3.2.1) ist, gilt für alle $x \in I$ (nach Definition 3.1.1):

$$
\begin{aligned}
& 0= y^{\prime \prime}(x)+p(x) \cdot y^{\prime}(x)+q(x) \cdot y(x) \\
&=[u(x)+\mathrm{i} \cdot v(x)]^{\prime \prime} \\
&+ p(x) \cdot[u(x)+\mathrm{i} \cdot v(x)]^{\prime} \\
& \quad+q(x) \cdot[u(x)+\mathrm{i} \cdot v(x)] \\
&=\left[u^{\prime \prime}(x)+p(x) \cdot u^{\prime}(x)+q(x) \cdot u(x)\right] \\
& \quad+\mathrm{i} \cdot\left[v^{\prime \prime}(x)+p(x) \cdot v^{\prime}(x)+q(x) \cdot v(x)\right] .
\end{aligned}
$$

Für jedes $x \in I$ liefert der Vergleich zweier komplexen Zahlen

$$
\left\{\begin{array}{l}
u^{\prime \prime}(x)+p(x) \cdot u^{\prime}(x)+q(x) \cdot u(x)=0 \\
v^{\prime \prime}(x)+p(x) \cdot v^{\prime}(x)+q(x) \cdot v(x)=0
\end{array}\right.
$$

Nach Definition 3.1.1 sind also die Funktionen $u, v$ Lösungen der homogenen Gleichung (3.2.1) auf $I$. 


\section{Beispiel 3.2.6}

Die komplexwertige Funktion $y: \mathbb{R} \rightarrow \mathbb{C}$ mit $y(x):=\mathrm{e}^{\mathrm{i} \cdot 2 x}$ ist eine Lösung der Gleichung

$$
y^{\prime \prime}(x)+4 \cdot y(x)=0
$$

auf $\mathbb{R}$. In der Tat gilt nach Beispiel 3.2.4 ii) für jedes $x \in \mathbb{R}$ :

$$
\begin{gathered}
y^{\prime}(x)=2 \mathrm{i} \cdot \mathrm{e}^{\mathrm{i} \cdot 2 x}, y^{\prime \prime}(x)=-4 \cdot \mathrm{e}^{\mathrm{i} \cdot 2 x} \text { und } \\
y^{\prime \prime}(x)+4 \cdot y(x)=-4 \cdot \mathrm{e}^{\mathrm{i} \cdot 2 x}+4 \cdot \mathrm{e}^{\mathrm{i} \cdot 2 x}=0 .
\end{gathered}
$$

Nach der EuLER'schen Formel gilt für jedes $x \in \mathbb{R}$ :

$$
y(x)=\mathrm{e}^{\mathrm{i} \cdot 2 x}=\cos (2 x)+\mathrm{i} \cdot \sin (2 x) .
$$

Für jedes $x \in \mathbb{R}$ seien $u(x):=\cos (2 x)$ und $v(x):=\sin (2 x)$. Dann gilt auf $\mathbb{R}$ :

$$
\begin{aligned}
u^{\prime}(x) & =-2 \sin (2 x), & v^{\prime}(x) & =2 \cos (2 x), \\
u^{\prime \prime}(x) & =-4 \cos (2 x), & v^{\prime \prime}(x) & =-4 \sin (2 x), \\
u^{\prime \prime}(x)+4 u(x) & =0, & v^{\prime \prime}(x)+4 v(x) & =0 .
\end{aligned}
$$

D.h., die Funktionen $u, v$ sind reellwertige Lösungen von $y^{\prime \prime}(x)+4 \cdot y(x)=0$ auf $\mathbb{R}$.

\section{Definition 3.2.7}

Seien die Funktionen $y_{1}, y_{2}: I \rightarrow \mathbb{R}$ auf einem Intervall I definiert und differenzierbar. Dann heißt die Determinante

$$
W(x):=\operatorname{det}\left(\begin{array}{ll}
y_{1}(x) & y_{2}(x) \\
y_{1}^{\prime}(x) & y_{2}^{\prime}(x)
\end{array}\right)=:\left|\begin{array}{ll}
y_{1}(x) & y_{2}(x) \\
y_{1}^{\prime}(x) & y_{2}^{\prime}(x)
\end{array}\right|
$$

WRONSKI-Determinante $^{4}$ dieser Funktionen.

\section{Definition 3.2.8}

Seien $y_{1}$ und $y_{2} z$ wei verschiedene Lösungen von (3.2.1). Dann heißt $\left\{y_{1}, y_{2}\right\}$ ein Fundamentalsystem von (3.2.1) auf I, falls für die WRONSKI-Determinante dieser Funktionen stets $W(x) \neq 0$ für alle $x \in I$ erfüllt ist.

${ }^{4}$ Josef Maria Hoëné-Wronski, 1776-1853 


\section{Beispiel 3.2.9}

$$
y^{\prime \prime}(x)-y(x)=0, \quad x \in \mathbb{R} .
$$

1) $y_{1}(x):=\mathrm{e}^{x}$ und $y_{2}(x):=\mathrm{e}^{-x}$ sind Lösungen dieser Gleichung auf $\mathbb{R}$. In der Tat gilt für jedes $x \in \mathbb{R}$ :

$$
\begin{aligned}
y_{1}^{\prime}(x) & =\mathrm{e}^{x}, & y_{2}^{\prime}(x) & =-\mathrm{e}^{-x}, \\
y_{1}^{\prime \prime}(x) & =\mathrm{e}^{x}, & & \mathrm{e}^{-x}, \\
y_{1}^{\prime \prime}(x)-y_{1}(x) & =\mathrm{e}^{x}-\mathrm{e}^{x}=0, & y_{2}^{\prime \prime}(x)-y_{2}(x) & =\mathrm{e}^{-x}-\mathrm{e}^{-x}=0 .
\end{aligned}
$$

2) Nach Definition 3.2.7 gilt für alle $x \in \mathbb{R}$ :

$$
W(x)=\left|\begin{array}{ll}
y_{1}(x) & y_{2}(x) \\
y_{1}^{\prime}(x) & y_{2}^{\prime}(x)
\end{array}\right|=\left|\begin{array}{cc}
\mathrm{e}^{x} & \mathrm{e}^{-x} \\
\mathrm{e}^{x} & -\mathrm{e}^{-x}
\end{array}\right|=-2 \neq 0 .
$$

Nach Definition 3.2.8 ist $\left\{\mathrm{e}^{x}, \mathrm{e}^{-x}\right\}$ ein Fundamentalsystem von $y^{\prime \prime}(x)-y(x)=0$ auf $\mathbb{R}$.

\section{Satz 3.2.10}

Seien $y_{1}$ und $y_{2} z$ wei verschiedene Lösungen von (3.2.1). Existiert ein $x_{0} \in I$ mit $W\left(x_{0}\right) \neq 0$, so gilt $W(x) \neq 0$ für alle $x \in I$.

\section{BeweIs:}

Da die Funktionen $y_{1}, y_{2}: I \rightarrow \mathbb{R}$ zwei verschiedene Lösungen der Gleichung (3.2.1) sind, gilt für jedes $x \in I$ und jedes $k \in\{1,2\}$ :

$$
\begin{aligned}
y_{k}^{\prime \prime}(x)+p(x) \cdot y_{k}^{\prime}(x)+q(x) \cdot y_{k}(x) & =0 \\
y_{k}^{\prime \prime}(x) & =-p(x) \cdot y_{k}^{\prime}(x)-q(x) \cdot y_{k}(x) .
\end{aligned}
$$

Wir untersuchen die Funktion $W: I \rightarrow \mathbb{R}$, wobei

$$
W(x):=\left|\begin{array}{ll}
y_{1}(x) & y_{2}(x) \\
y_{1}^{\prime}(x) & y_{2}^{\prime}(x)
\end{array}\right|=y_{1}(x) \cdot y_{2}^{\prime}(x)-y_{1}^{\prime}(x) \cdot y_{2}(x)
$$

WronsKI-Determinante der Funktionen $y_{1}, y_{2}$ auf $I$ ist. 
Die Funktion $W$ ist auf $I$ differenzierbar und für jedes $x \in I$ gilt:

$$
\begin{aligned}
W^{\prime}(x)= & {\left[y_{1}(x) \cdot y_{2}^{\prime}(x)-y_{1}^{\prime}(x) \cdot y_{2}(x)\right]^{\prime} } \\
= & y_{1}^{\prime}(x) \cdot y_{2}^{\prime}(x)+y_{1}(x) \cdot y_{2}^{\prime \prime}(x)-y_{1}^{\prime \prime}(x) \cdot y_{2}(x)-y_{1}^{\prime}(x) \cdot y_{2}^{\prime}(x) \\
= & y_{1}(x) \cdot y_{2}^{\prime \prime}(x)-y_{1}^{\prime \prime}(x) \cdot y_{2}(x) \\
& \stackrel{\text { s.o. }}{=} y_{1}(x) \cdot\left[-p(x) \cdot y_{2}^{\prime}(x)-q(x) \cdot y_{2}(x)\right] \\
& \quad-\left[-p(x) \cdot y_{1}^{\prime}(x)-q(x) \cdot y_{1}(x)\right] \cdot y_{2}(x) \\
& =-p(x) \cdot y_{1}(x) \cdot y_{2}^{\prime}(x)+p(x) \cdot y_{1}^{\prime}(x) \cdot y_{2}(x) \\
= & -p(x) \cdot\left[y_{1}(x) \cdot y_{2}^{\prime}(x)-y_{1}^{\prime}(x) \cdot y_{2}(x)\right] \\
= & -p(x) \cdot W(x) .
\end{aligned}
$$

Das Anfangswertproblem

$$
\left\{\begin{array}{l}
W^{\prime}(x)=-p(x) \cdot W(x), \\
W\left(x_{0}\right)=W_{0} \quad\left(\text { mit } W_{0} \in \mathbb{R}\right) .
\end{array}\right.
$$

ist auf $I$ eindeutig lösbar (vgl. Folgerung 1.2.5) und es gilt für jedes $x \in I$ :

$$
W(x)=W\left(x_{0}\right) \cdot \mathrm{e}^{-\int_{x_{0}}^{x} p(t) \mathrm{d} t} .
$$

Somit folgt: Ist $W\left(x_{0}\right) \neq 0$, dann gilt $W(x) \neq 0$ für alle $x \in I$.

\section{Satz und Definition 3.2.11}

Allgemeine Lösung der linearen homogenen Differentialgleichung 2. Ordnung.

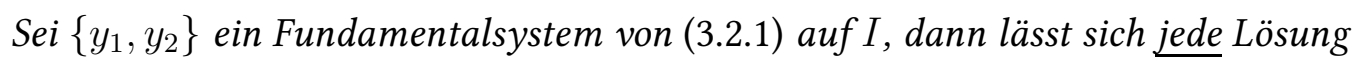
$y: I \rightarrow \mathbb{R}$ von (3.2.1) in der Form

$$
y(x)=c_{1} \cdot y_{1}(x)+c_{2} \cdot y_{2}(x)
$$

darstellen, wobei die Koeffizienten $c_{1}, c_{2} \in \mathbb{R}$ eindeutig bestimmt sind.

Lässt man in $c_{1} \cdot y_{1}(x)+c_{2} \cdot y_{2}(x)$ die Koeffizienten $c_{1}, c_{2}$ unabhängig von einander die Menge der reellen Zahlen durchlaufen, so erhält man alle Lösungen der Gleichung (3.2.1). 
Man sagt: Durch

$$
y(x)=c_{1} \cdot y_{1}(x)+c_{2} \cdot y_{2}(x)
$$

ist die allgemeine Lösung der linearen homogenen Differentialgleichung 2. Ordnung

$$
y^{\prime \prime}(x)+p(x) \cdot y^{\prime}(x)+q(x) \cdot y(x)=0
$$

auf I gegeben.

BeweIs:

Da $\left\{y_{1}, y_{2}\right\}$ ein Fundamentalsystem von (3.2.1) ist, ist nach Definition 3.2.8 und Satz 3.2.1 jede Funktion $y: I \rightarrow \mathbb{R}$ mit

$$
y(x):=c_{1} \cdot y_{1}(x)+c_{2} \cdot y_{2}(x)
$$

immer eine Lösung von (3.2.1) für beliebige $c_{1}, c_{2} \in \mathbb{R}$.

Sei $\widetilde{y}: I \rightarrow \mathbb{R}$ eine beliebige Lösung von (3.2.1). Wir zeigen: Es existieren $c_{1}, c_{2} \in \mathbb{R}$, sodass auf $I$ gilt:

$$
\widetilde{y}(x)=c_{1} \cdot y_{1}(x)+c_{2} \cdot y_{2}(x) .
$$

Dazu nehmen wir ein beliebiges $x_{0} \in I$ und bestimmen $\widetilde{y}\left(x_{0}\right)$ und $\widetilde{y}^{\prime}\left(x_{0}\right)$. Wir suchen also $c_{1}, c_{2} \in \mathbb{R}$ mit:

$$
\left\{\begin{array}{l}
c_{1} \cdot y_{1}\left(x_{0}\right)+c_{2} \cdot y_{2}\left(x_{0}\right)=\widetilde{y}\left(x_{0}\right), \\
c_{1} \cdot y_{1}^{\prime}\left(x_{0}\right)+c_{2} \cdot y_{2}^{\prime}\left(x_{0}\right)=\widetilde{y}^{\prime}\left(x_{0}\right) .
\end{array}\right.
$$

Weiter gilt

$$
\triangle:=\left|\begin{array}{ll}
y_{1}\left(x_{0}\right) & y_{2}\left(x_{0}\right) \\
y_{1}^{\prime}\left(x_{0}\right) & y_{2}^{\prime}\left(x_{0}\right)
\end{array}\right|=W\left(x_{0}\right) \neq 0,
$$

da $\left\{y_{1}, y_{2}\right\}$ ein Fundamentalsystem von (3.2.1) ist. Dann ist das LGS $(*)$ eindeutig für $c_{1}, c_{2}$ lösbar. Sei $\left\{\widetilde{c_{1}}, \widetilde{c_{2}}\right\}$ diese eindeutige Lösung des LGS $(*)$. Wir untersuchen die Funktion $y: I \rightarrow \mathbb{R}$ mit

$$
y(x):=\widetilde{c_{1}} \cdot y_{1}(x)+\widetilde{c_{2}} \cdot y_{2}(x)
$$

Nach Satz 3.2.1 ist $y$ eine Lösung von (3.2.1) auf $I$ und

$$
y\left(x_{0}\right)=\widetilde{c_{1}} \cdot y_{1}\left(x_{0}\right)+\widetilde{c_{2}} \cdot y_{2}\left(x_{0}\right)=\widetilde{y}\left(x_{0}\right),
$$


sowie

$$
y^{\prime}\left(x_{0}\right)=\widetilde{c_{1}} \cdot y_{1}^{\prime}\left(x_{0}\right)+\widetilde{c_{2}} \cdot y_{2}^{\prime}\left(x_{0}\right)=\widetilde{y}^{\prime}\left(x_{0}\right)
$$

Wegen der Eindeutigkeit der Lösung des Anfangswertproblems (Satz 3.1.2) gilt für alle $x \in I$ :

$$
y(x)=\widetilde{y}(x)
$$

und

$$
\widetilde{y}(x)=\widetilde{c_{1}} \cdot y_{1}(x)+\widetilde{c_{2}} \cdot y_{2}(x)
$$

Dann ist jede Lösung von (3.2.1) in der Form $c_{1} \cdot y_{1}(x)+c_{2} \cdot y_{2}(x)$ mit $c_{1}, c_{2} \in \mathbb{R}$ darstellbar.

Beispiel 3.2.12

$$
y^{\prime \prime}(x)+y(x)=0, \quad x \in \mathbb{R}
$$

1) $y_{1}(x):=\cos (x)$ und $y_{2}(x):=\sin (x)$ sind Lösungen dieser Gleichung auf $\mathbb{R}$. In der Tat gilt für jedes $x \in \mathbb{R}$ :

$$
\begin{aligned}
y_{1}^{\prime}(x) & =-\sin (x), & y_{2}^{\prime}(x) & =\cos (x), \\
y_{1}^{\prime \prime}(x) & =-\cos (x), & y_{2}^{\prime \prime}(x) & =-\sin (x), \\
y_{1}^{\prime \prime}(x)+y_{1}(x) & =0, & y_{2}^{\prime \prime}(x)+y_{2}(x) & =0 .
\end{aligned}
$$

2) Nach Definition 3.2.7 gilt für alle $x \in \mathbb{R}$ :

$$
\begin{aligned}
W(x) & =\left|\begin{array}{ll}
y_{1}(x) & y_{2}(x) \\
y_{1}^{\prime}(x) & y_{2}^{\prime}(x)
\end{array}\right|=\left|\begin{array}{cc}
\cos (x) & \sin (x) \\
-\sin (x) & \cos (x)
\end{array}\right|=\cos ^{2}(x)+\sin ^{2}(x) \\
& =1 \neq 0
\end{aligned}
$$

Nach Definition 3.2.8 ist somit $\{\cos (x), \sin (x)\}$ ein Fundamentalsystem von $y^{\prime \prime}(x)+y(x)=0$ auf $\mathbb{R}$.

3) Die allgemeine Lösung der Gleichung $y^{\prime \prime}(x)+y(x)=0$ auf $\mathbb{R}$ lautet also nach Satz 3.2.11:

$$
y(x)=c_{1} \cdot \cos (x)+c_{2} \cdot \sin (x) \quad \text { mit beliebigen } \quad c_{1}, c_{2} \in \mathbb{R} .
$$




\section{Satz 3.2.13}

Sei $y_{1}$ eine Lösung von (3.2.1), sodass $y_{1}(x) \neq 0$ für alle $x \in I$. Dann kann man eine zweite Lösung $y_{2}$ der Gleichung (3.2.1) bestimmen, sodass $\left\{y_{1}, y_{2}\right\}$ ein Fundamentalsystem von (3.2.1) bildet.

BEwEIs:

Wir suchen die Lösung $y_{2}$ in der Form $y_{2}(x)=y_{1}(x) \cdot u(x)$, wobei $u: I \rightarrow \mathbb{R}$ eine unbekannte zweimal differenzierbare Funktion ist. Dann gilt:

$$
\begin{aligned}
& y_{2}^{\prime}(x)=y_{1}^{\prime}(x) \cdot u(x)+y_{1}(x) \cdot u^{\prime}(x), \\
& y_{2}^{\prime \prime}(x)=y_{1}^{\prime \prime}(x) \cdot u(x)+2 y_{1}^{\prime}(x) \cdot u^{\prime}(x)+y_{1}(x) \cdot u^{\prime \prime}(x) .
\end{aligned}
$$

In die Differentialgleichung (3.2.1) eingesetzt ergibt:

$$
\begin{gathered}
y_{1}^{\prime \prime}(x) \cdot u(x)+2 y_{1}^{\prime}(x) \cdot u^{\prime}(x)+y_{1}(x) \cdot u^{\prime \prime}(x) \\
+p(x) \cdot\left(y_{1}^{\prime}(x) \cdot u(x)+y_{1}(x) \cdot u^{\prime}(x)\right)+q(x) \cdot y_{1}(x) \cdot u(x)=0 \\
\Leftrightarrow \quad \underbrace{\left(y^{\prime \prime}(x)+p(x) \cdot y_{1}^{\prime}(x)+q(x) \cdot y_{1}(x)\right.}_{=0}) \cdot u(x) \\
+y_{1}(x) \cdot u^{\prime \prime}(x)+\left(2 y_{1}^{\prime}(x)+p(x) \cdot y_{1}(x)\right) \cdot u^{\prime}(x)=0,
\end{gathered}
$$

da $y_{1}$ eine Lösung von (3.2.1) ist. Die unbekannte Funktion $u$ genügt also der Differentialgleichung

$$
\begin{aligned}
& y_{1}(x) \cdot u^{\prime \prime}(x)+\left(2 y_{1}^{\prime}(x)+p(x) \cdot y_{1}(x)\right) \cdot u^{\prime}(x)=0 \\
& \underset{y_{1}(x) \neq 0}{\Leftrightarrow} \\
& u^{\prime \prime}(x)+\left(\frac{2 y_{1}^{\prime}(x)}{y_{1}(x)}+p(x)\right) \cdot u^{\prime}(x)=0 .
\end{aligned}
$$

Mit anderen Worten genügt $u^{\prime}$ einer linearen homogenen Differentialgleichung erster Ordnung auf $I$. Bezeichnet $P: I \rightarrow \mathbb{R}$ eine Stammfunktion von $p$, so ist $\bar{P}(x):=\ln \left(\left(y_{1}(x)\right)^{2}\right)+P(x)$ eine Stammfunktion von $\frac{2 y_{1}^{\prime}(x)}{y_{1}(x)}+p(x)$. Nach Bemerkung 1.2.2 ist dann

$$
u^{\prime}(x):=\mathrm{e}^{-\ln \left(\left(y_{1}(x)\right)^{2}\right)-P(x)}=\frac{\mathrm{e}^{-P(x)}}{\left(y_{1}(x)\right)^{2}}
$$

eine Lösung von (3.2.5) auf $I$ und wir erhalten $u$ als eine Stammfunktion von $u^{\prime}$. Mit dieser Funktion $u$ ist also $y_{2}(x)=y_{1}(x) \cdot u(x)$ eine (weitere) Lösung der Gleichung (3.2.1) auf $I$. 
Nach Definition 3.2.7 erhalten wir ferner für alle $x \in I$ :

$$
\begin{aligned}
W(x) & =\left|\begin{array}{ll}
y_{1}(x) & y_{2}(x) \\
y_{1}^{\prime}(x) & y_{2}^{\prime}(x)
\end{array}\right|=\left|\begin{array}{cc}
y_{1}(x) & y_{1}(x) \cdot u(x) \\
y_{1}^{\prime}(x) & y_{1}^{\prime}(x) \cdot u(x)+y_{1}(x) \cdot u^{\prime}(x)
\end{array}\right| \\
& =y_{1}(x) \cdot\left(y_{1}^{\prime}(x) \cdot u(x)+y_{1}(x) \cdot u^{\prime}(x)\right)-y_{1}^{\prime}(x) \cdot y_{1}(x) \cdot u(x) \\
& =\left(y_{1}(x)\right)^{2} \cdot u^{\prime}(x)=\mathrm{e}^{-P(x)} \neq 0 .
\end{aligned}
$$

Somit ist $\left\{y_{1}, y_{2}\right\}$ nach Definition 3.2.8 ein Fundamentalsystem der Gleichung (3.2.1) auf $I$.

\section{Beispiel 3.2.14}

$$
y^{\prime \prime}(x)-2 y^{\prime}(x)+y(x)=0, \quad x \in \mathbb{R} .
$$

1) Wir suchen zunächst eine komplexwertige Lösung $y_{1}$ in der Form $y_{1}(x):=\mathrm{e}^{\lambda x}$ mit $\lambda \in \mathbb{C}$. Dann gilt nach Beispiel 3.2.4 für jedes $x \in \mathbb{R}$ :

$$
\begin{aligned}
& y_{1}^{\prime}(x)=\lambda \cdot \mathrm{e}^{\lambda x}, \quad y_{1}^{\prime \prime}(x)=\lambda^{2} \cdot \mathrm{e}^{\lambda x} \\
& y_{1}^{\prime \prime}(x)-2 y_{1}^{\prime}(x)+y_{1}(x)=0 \quad \Leftrightarrow \quad\left(\lambda^{2}-2 \lambda+1\right) \cdot \mathrm{e}^{\lambda x}=0 \\
& \underset{\mathrm{e}^{\lambda x \neq 0}}{\Longleftrightarrow} \quad \lambda^{2}-2 \lambda+1=0 \quad \Leftrightarrow \quad(\lambda-1)^{2}=0 \quad \Leftrightarrow \quad \lambda=1 .
\end{aligned}
$$

Also ist $y_{1}(x)=\mathrm{e}^{x}$ eine reellwertige Lösung der Gleichung (3.2.6) auf $\mathbb{R}$.

2) Wir suchen eine weitere Lösung $y_{2}$ in der Form $y_{2}(x):=\mathrm{e}^{x} \cdot u(x)$, wobei $u: \mathbb{R} \rightarrow \mathbb{R}$ eine neue unbekannte Funktion ist. Für jedes $x \in \mathbb{R}$ gilt:

$$
\begin{aligned}
y_{2}^{\prime}(x) & =\left(\mathrm{e}^{x} \cdot u(x)\right)^{\prime}=\mathrm{e}^{x} \cdot u(x)+\mathrm{e}^{x} \cdot u^{\prime}(x) \quad \text { und } \\
y_{2}^{\prime \prime}(x) & =\left(\mathrm{e}^{x} \cdot u(x)+\mathrm{e}^{x} \cdot u^{\prime}(x)\right)^{\prime} \\
& =\mathrm{e}^{x} \cdot u(x)+\mathrm{e}^{x} \cdot u^{\prime}(x)+\mathrm{e}^{x} \cdot u^{\prime}(x)+\mathrm{e}^{x} \cdot u^{\prime \prime}(x) \\
& =\mathrm{e}^{x} \cdot u(x)+2 \mathrm{e}^{x} \cdot u^{\prime}(x)+\mathrm{e}^{x} \cdot u^{\prime \prime}(x) .
\end{aligned}
$$

Mit der Bedingung $y_{2}^{\prime \prime}(x)-2 y_{2}^{\prime}(x)+y_{2}(x)=0$ erhalten wir

$$
\begin{aligned}
& \mathrm{e}^{x} \cdot u(x)+2 \mathrm{e}^{x} \cdot u^{\prime}(x)+\mathrm{e}^{x} \cdot u^{\prime \prime}(x) \\
& -2\left(\mathrm{e}^{x} \cdot u(x)+\mathrm{e}^{x} \cdot u^{\prime}(x)\right)+\mathrm{e}^{x} \cdot u(x)=0 \\
& \Longleftrightarrow \quad \mathrm{e}^{x} \cdot u^{\prime \prime}(x)=0 \quad \underset{\mathrm{e}^{x} \neq 0}{\Longleftrightarrow} \quad u^{\prime \prime}(x)=0 \\
& \Longleftrightarrow \quad u(x)=m \cdot x+n \quad \text { mit beliebigen } \quad m, n \in \mathbb{R} \text {. }
\end{aligned}
$$


Wir nehmen (z.B.) $n=0$ und $m=1$, woraus $u(x)=x$ und somit $y_{2}(x)=x \cdot \mathrm{e}^{x}$ für alle $x \in \mathbb{R}$ folgt. Also ist $y_{2}(x)=x \cdot \mathrm{e}^{x}$ auch eine Lösung der Gleichung (3.2.6) auf $\mathbb{R}$.

3) Nach Definition 3.2.7 gilt für alle $x \in \mathbb{R}$ :

$$
\begin{aligned}
W(x) & =\left|\begin{array}{ll}
y_{1}(x) & y_{2}(x) \\
y_{1}^{\prime}(x) & y_{2}^{\prime}(x)
\end{array}\right|=\left|\begin{array}{cc}
\mathrm{e}^{x} & x \cdot \mathrm{e}^{x} \\
\mathrm{e}^{x} & \mathrm{e}^{x}+x \cdot \mathrm{e}^{x}
\end{array}\right|=\mathrm{e}^{x} \cdot\left(\mathrm{e}^{x}+x \cdot \mathrm{e}^{x}\right)-\mathrm{e}^{x} \cdot x \cdot \mathrm{e}^{x} \\
& =\mathrm{e}^{2 x} \neq 0 .
\end{aligned}
$$

Nach Definition 3.2.8 ist somit $\left\{\mathrm{e}^{x}, x \cdot \mathrm{e}^{x}\right\}$ ein Fundamentalsystem der Gleichung (3.2.6) auf $\mathbb{R}$.

4) Die allgemeine Lösung der Gleichung (3.2.6) auf $\mathbb{R}$ lautet nach Satz 3.2.11:

$$
y(x)=c_{1} \cdot \mathrm{e}^{x}+c_{2} \cdot x \cdot \mathrm{e}^{x} \quad \text { mit beliebigen } \quad c_{1}, c_{2} \in \mathbb{R} .
$$

\section{Satz 3.2.15}

Die Funktionen $p, q: I \rightarrow \mathbb{R}$ seien auf einem Intervall I definiert und stetig. Dann besitzt die lineare homogene Differentialgleichung 2. Ordnung

$$
y^{\prime \prime}(x)+p(x) \cdot y^{\prime}(x)+q(x) \cdot y(x)=0
$$

ein Fundamentalsystem auf I.

BeweIs:

Wir wählen ein beliebiges $x_{0} \in I$ und finden zwei (spezielle) Lösungen $y_{1}$ und $y_{2}$ der homogenen Differentialgleichung (3.2.1) mit folgenden Anfangsbedingungen:

$$
\left\{\begin{array} { l } 
{ y _ { 1 } ( x _ { 0 } ) = 1 } \\
{ y _ { 1 } ^ { \prime } ( x _ { 0 } ) = 0 }
\end{array} \quad \text { bzw. } \quad \left\{\begin{array}{l}
y_{2}\left(x_{0}\right)=0 \\
y_{2}^{\prime}\left(x_{0}\right)=1
\end{array}\right.\right.
$$

Nach Satz 3.1.2 sind die Funktionen $y_{1}$ und $y_{2}$ im ganzen Intervall $I$ eindeutig bestimmt. An der Stelle $x_{0}$ erhalten wir für die Wronski-Determinante dieser Funktionen:

$$
W\left(x_{0}\right)=\left|\begin{array}{ll}
y_{1}\left(x_{0}\right) & y_{2}\left(x_{0}\right) \\
y_{1}^{\prime}\left(x_{0}\right) & y_{2}^{\prime}\left(x_{0}\right)
\end{array}\right|=\left|\begin{array}{ll}
1 & 0 \\
0 & 1
\end{array}\right|=1 \neq 0 .
$$

Nach Satz 3.2.10 ist $W(x) \neq 0$ für alle $x \in I$ und nach Definition 3.2.8 ist somit $\left\{y_{1}, y_{2}\right\}$ ein Fundamentalsystem von (3.2.1) auf $I$. 


\subsection{Lineare homogene Differentialgleichung zwei- ter Ordnung mit konstanten Koeffizienten}

\section{Definition 3.3.1}

Gegeben seien $p, q \in \mathbb{R}$. Gesucht ist eine Funktion $y: \mathbb{R} \rightarrow \mathbb{R}$, sodass für alle $x \in \mathbb{R}$ gilt

$$
y^{\prime \prime}(x)+p \cdot y^{\prime}(x)+q \cdot y(x)=0 .
$$

Die Gleichung (3.3.1) heißt lineare homogene Differentialgleichung zweiter Ordnung mit konstanten Koeffizienten. Die Funktion $y: \mathbb{R} \rightarrow \mathbb{R}$ heißt Lösung der Gleichung (3.3.1) auf $\mathbb{R}$.

Eine Funktion $y: \mathbb{R} \rightarrow \mathbb{C}$, die (3.3.1) erfüllt, heißt komplexwertige Lösung der Gleichung (3.3.1) auf $\mathbb{R}$.

\section{Bemerkung und Definition 3.3.2}

Wir suchen Lösungen der Gleichung (3.3.1) in der Form

$$
y(x)=\mathrm{e}^{\lambda x}
$$

mit $\lambda \in \mathbb{C}$. Nach Beispiel 3.2.4 gilt für jedes $x \in \mathbb{R}$ :

$$
y^{\prime}(x)=\lambda \cdot \mathrm{e}^{\lambda x}, \quad y^{\prime \prime}(x)=\lambda^{2} \cdot \mathrm{e}^{\lambda x} .
$$

In Gleichung (3.3.1) eingesetzt:

$$
\lambda^{2} \cdot \mathrm{e}^{\lambda x}+p \cdot \lambda \cdot \mathrm{e}^{\lambda x}+q \cdot \mathrm{e}^{\lambda x}=0
$$

liefert unter Berücksichtigung, dass $\mathrm{e}^{\lambda x} \neq 0$ für alle $x \in \mathbb{R}$ und alle $\lambda \in \mathbb{C}$ :

$$
\begin{gathered}
\lambda^{2}+p \cdot \lambda+q=0 \\
\Leftrightarrow \quad \lambda=-\frac{p}{2} \pm \sqrt{\left(\frac{p}{2}\right)^{2}-q .}
\end{gathered}
$$

Gleichung (3.3.1c) heißt charakteristische Gleichung der Differentialgleichung (3.3.1) und $D=\left(\frac{p}{2}\right)^{2}-q$ die Diskriminante der charakteristischen Gleichung (3.3.1c).

Es gilt: Die (komplexwertige) Funktion $y: \mathbb{R} \rightarrow \mathbb{C}$ mit $y(x):=\mathrm{e}^{\lambda x}$ und $\lambda \in \mathbb{C}$ ist genau dann eine (komplexwertige) Lösung von (3.3.1) auf $\mathbb{R}$, wenn $\lambda$ eine (komplexe) Lösung der zugehörigen charakteristischen Gleichung (3.3.1c) ist. 
$\underline{\text { I. Fall }}\left(\frac{p}{2}\right)^{2}-q>0$

Seien $\lambda_{1}:=-\frac{p}{2}+\sqrt{\left(\frac{p}{2}\right)^{2}-q} ; \quad \lambda_{2}:=-\frac{p}{2}-\sqrt{\left(\frac{p}{2}\right)^{2}-q}$.

Dann sind $\left\{\lambda_{1}, \lambda_{2}\right\}$ zwei verschiedene reelle Lösungen der charakteristischen Gleichung (3.3.1c).

Die reellwertigen Funktionen $y_{1}, y_{2}: \mathbb{R} \rightarrow \mathbb{R}$ mit $y_{1}(x):=\mathrm{e}^{\lambda_{1} x}$ und $y_{2}(x):=\mathrm{e}^{\lambda_{2} x}$ bilden ein Fundamentalsystem von (3.3.1) auf $\mathbb{R}$. In der Tat:

Die Funktionen $y_{1}, y_{2}$ sind Lösungen von (3.3.1) und für alle $x \in \mathbb{R}$ gilt:

$$
\begin{aligned}
W(x) & =\left|\begin{array}{ll}
y_{1}\left(x_{0}\right) & y_{2}\left(x_{0}\right) \\
y_{1}^{\prime}\left(x_{0}\right) & y_{2}^{\prime}\left(x_{0}\right)
\end{array}\right|=\left|\begin{array}{cc}
\mathrm{e}^{\lambda_{1} x} & \mathrm{e}^{\lambda_{2} x} \\
\lambda_{1} \cdot \mathrm{e}^{\lambda_{1} x} & \lambda_{2} \cdot \mathrm{e}^{\lambda_{2} x}
\end{array}\right| \\
& =\mathrm{e}^{\lambda_{1} x} \cdot \lambda_{2} \cdot \mathrm{e}^{\lambda_{2} x}-\lambda_{1} \cdot \mathrm{e}^{\lambda_{1} x} \cdot \mathrm{e}^{\lambda_{2} x}=\left(\lambda_{2}-\lambda_{1}\right) \cdot \mathrm{e}^{\lambda_{1} x+\lambda_{2} x} \neq 0,
\end{aligned}
$$

da $\lambda_{1} \neq \lambda_{2}$.

\section{Folgerung 3.3.3}

Nach Satz 3.2.11 ist die Funktion $y: \mathbb{R} \rightarrow \mathbb{R}$ mit

$$
y(x):=c_{1} \cdot \mathrm{e}^{\lambda_{1} x}+c_{2} \cdot \mathrm{e}^{\lambda_{2} x}
$$

und beliebigen $c_{1}, c_{2} \in \mathbb{R}$ die allgemeine Lösung von (3.3.1) im Fall $\left(\frac{p}{2}\right)^{2}-q>0$.

\section{Beispiel 3.3.4}

$$
y^{\prime \prime}(x)-2 y^{\prime}(x)-3 y(x)=0, \quad x \in \mathbb{R} .
$$

Die entsprechende charakteristische Gleichung ist

$$
\lambda^{2}-2 \lambda-3=0 \quad \Leftrightarrow \quad \lambda=-1 \quad \text { oder } \quad \lambda=3 .
$$

Seien $\lambda_{1}:=-1$ und $\lambda_{2}:=3$. Dann bilden die Funktionen $y_{1}, y_{2}: \mathbb{R} \rightarrow \mathbb{R}$ mit

$$
y_{1}(x):=\mathrm{e}^{-x} \quad \text { und } \quad y_{2}(x):=\mathrm{e}^{3 x}
$$

ein Fundamentalsystem der Gleichung $\quad y^{\prime \prime}(x)-2 y^{\prime}(x)-3 y(x)=0 \quad$ auf $\mathbb{R}$. Nach Folgerung 3.3.3 ist

$$
y(x)=c_{1} \cdot \mathrm{e}^{-x}+c_{2} \cdot \mathrm{e}^{3 x}
$$

mit beliebigen $c_{1}, c_{2} \in \mathbb{R}$ die allgemeine Lösung dieser Gleichung auf $\mathbb{R}$. 


\section{$\underline{\text { II. Fall }}\left(\frac{p}{2}\right)^{2}-q=0$}

Die reelle Zahl $\lambda:=-\frac{p}{2}$ ist eine Lösung der charakteristischen Gleichung (3.3.1c) (mit Vielfachheit $r=2$ ). Die reellwertige Funktion $y_{1}: \mathbb{R} \rightarrow \mathbb{R}$ mit $y_{1}(x):=\mathrm{e}^{\lambda x}$ und $\lambda=-\frac{p}{2}$ ist eine Lösung von (3.3.1). Wir suchen eine weitere Lösung $y_{2}: \mathbb{R} \rightarrow \mathbb{R}$ in der Form

$$
y_{2}(x):=\mathrm{e}^{\lambda x} \cdot u(x),
$$

wobei $u: \mathbb{R} \rightarrow \mathbb{R}$ eine neue unbekannte Funktion ist. Für jedes $x \in \mathbb{R}$ gilt:

$$
\begin{aligned}
& y_{2}^{\prime}(x)=\left[\mathrm{e}^{\lambda x} \cdot u(x)\right]^{\prime}=\lambda \cdot \mathrm{e}^{\lambda x} \cdot u(x)+\mathrm{e}^{\lambda x} \cdot u^{\prime}(x), \\
& y_{2}^{\prime \prime}(x)=\lambda^{2} \cdot \mathrm{e}^{\lambda x} \cdot u(x)+2 \lambda \cdot \mathrm{e}^{\lambda x} \cdot u^{\prime}(x)+\mathrm{e}^{\lambda x} \cdot u^{\prime \prime}(x) .
\end{aligned}
$$

Dann gilt:

$$
\begin{aligned}
& y_{2}^{\prime \prime}(x)+p \cdot y_{2}^{\prime}(x)+q \cdot y_{2}(x)=0 \\
& \Longleftrightarrow \lambda^{2} \cdot \mathrm{e}^{\lambda x} \cdot u(x)+2 \lambda \cdot \mathrm{e}^{\lambda x} \cdot u^{\prime}(x)+\mathrm{e}^{\lambda x} \cdot u^{\prime \prime}(x) \\
& +p \cdot\left[\lambda \cdot \mathrm{e}^{\lambda x} \cdot u(x)+\mathrm{e}^{\lambda x} \cdot u^{\prime}(x)\right]+q \cdot \mathrm{e}^{\lambda x} \cdot u(x)=0 \\
& \Longleftrightarrow \quad\left[\left(\lambda^{2}+p \cdot \lambda+q\right) \cdot u(x)+(2 \lambda+p) \cdot u^{\prime}(x)+u^{\prime \prime}(x)\right] \cdot \mathrm{e}^{\lambda x}=0 \\
& \underset{\mathrm{e}^{\lambda x \neq 0}}{\Longleftrightarrow} \quad \underbrace{\left(\lambda^{2}+p \cdot \lambda+q\right)}_{=0} \cdot u(x)+\underbrace{(2 \lambda+p)}_{=0} \cdot u^{\prime}(x)+u^{\prime \prime}(x)=0 \text {, }
\end{aligned}
$$

da $\lambda=-\frac{p}{2}(\Leftrightarrow 2 \lambda+p=0)$ eine Lösung der charakteristischen Gleichung ist. Es folgt

$$
u^{\prime \prime}(x)=0 \quad \Leftrightarrow \quad u(x)=m \cdot x+n \quad \text { mit beliebigen } \quad m, n \in \mathbb{R} .
$$

Wir nehmen (z.B.) $n=0$ und $m=1$, woraus $u(x)=x$ und somit

$$
y_{2}(x)=x \cdot \mathrm{e}^{\lambda x}
$$

sich ergibt.

Die Funktionen $y_{1}, y_{2}: \mathbb{R} \rightarrow \mathbb{R}$ mit $y_{1}(x):=\mathrm{e}^{\lambda x}$ und $y_{2}(x):=x \cdot \mathrm{e}^{\lambda x}$ (wobei $\lambda=-\frac{p}{2}$ ist) bilden ein Fundamentalsystem von (3.3.1) auf $\mathbb{R}$. In der Tat gilt für alle $x \in \mathbb{R}$ :

$$
W(x)=\left|\begin{array}{ll}
y_{1}(x) & y_{2}(x) \\
y_{1}^{\prime}(x) & y_{2}^{\prime}(x)
\end{array}\right|=\left|\begin{array}{cc}
\mathrm{e}^{\lambda x} & x \cdot \mathrm{e}^{\lambda x} \\
\lambda \cdot \mathrm{e}^{\lambda x} & \mathrm{e}^{\lambda x}+\lambda x \cdot \mathrm{e}^{\lambda x}
\end{array}\right|=\mathrm{e}^{2 \lambda x} \neq 0 .
$$




\section{Folgerung 3.3.5}

Nach Satz 3.2.11 ist die Funktion $y: \mathbb{R} \rightarrow \mathbb{R}$ mit

$$
y(x):=c_{1} \cdot \mathrm{e}^{\lambda x}+c_{2} \cdot x \cdot \mathrm{e}^{\lambda x}=\mathrm{e}^{\lambda x} \cdot\left(c_{1}+c_{2} \cdot x\right)
$$

und beliebigen $c_{1}, c_{2} \in \mathbb{R}$ die allgemeine Lösung von (3.3.1) im Fall $\left(\frac{p}{2}\right)^{2}-q=0$.

\section{Beispiel 3.3.6}

$$
y^{\prime \prime}(x)+6 y^{\prime}(x)+9 y(x)=0, \quad x \in \mathbb{R} .
$$

Die entsprechende charakteristische Gleichung ist

$$
\lambda^{2}+6 \lambda+9=0 \quad \Leftrightarrow \quad \lambda=-3 \quad \text { (mit Vielfachheit } r=2 \text { ). }
$$

Dann bilden die Funktionen $y_{1}, y_{2}: \mathbb{R} \rightarrow \mathbb{R}$ mit

$$
y_{1}:=\mathrm{e}^{-3 x} \quad \text { und } \quad y_{2}:=x \cdot \mathrm{e}^{-3 x}
$$

ein Fundamentalsystem der Gleichung $y^{\prime \prime}(x)+6 y^{\prime}(x)+9 y(x)=0 \quad$ auf $\mathbb{R}$. Nach Folgerung 3.3.5 ist

$$
y(x)=\mathrm{e}^{-3 x} \cdot\left(c_{1}+c_{2} \cdot x\right)
$$

mit beliebigen $c_{1}, c_{2} \in \mathbb{R}$ die allgemeine Lösung dieser Gleichung auf $\mathbb{R}$.

$\underline{\text { III. Fall }}\left(\frac{p}{2}\right)^{2}-q<0 \quad \Leftrightarrow \quad q-\left(\frac{p}{2}\right)^{2}>0$

Wegen

$$
\begin{aligned}
\lambda & =-\frac{p}{2} \pm \sqrt{\left(\frac{p}{2}\right)^{2}-q}=-\frac{p}{2} \pm \sqrt{-\left(q-\left(\frac{p}{2}\right)^{2}\right)} \\
& =-\frac{p}{2} \pm \mathrm{i} \cdot \sqrt{q-\left(\frac{p}{2}\right)^{2}}
\end{aligned}
$$

sind $\lambda=\alpha \pm \mathrm{i} \cdot \beta$ mit $\alpha:=-\frac{p}{2}$ und $\beta:=\sqrt{q-\left(\frac{p}{2}\right)^{2}}$ zwei verschiedene komplexe Lösungen der charakteristischen Gleichung (3.3.1c). 
Die komplexwertigen Funktionen $y_{1}^{\star}, y_{2}^{\star}: \mathbb{R} \rightarrow \mathbb{C}$ mit

$$
y_{1}^{\star}(x):=\mathrm{e}^{(\alpha+\mathrm{i} \cdot \beta) x} \stackrel{\text { Bsp 3.2.4 }}{=} \mathrm{e}^{\alpha x} \cdot(\cos (\beta x)+\mathrm{i} \cdot \sin (\beta x))
$$

und

$$
\begin{aligned}
& y_{2}^{\star}(x):=\mathrm{e}^{(\alpha-\mathrm{i} \cdot \beta) x}=\mathrm{e}^{(\alpha+\mathrm{i} \cdot(-\beta)) x} \\
& \stackrel{\text { Bsp 3.2.4 }}{=} \mathrm{e}^{\alpha x} \cdot(\cos ((-\beta) x)+\mathrm{i} \cdot \sin ((-\beta) x)) \\
&=\mathrm{e}^{\alpha x} \cdot(\cos (\beta x)-\mathrm{i} \cdot \sin (\beta x))
\end{aligned}
$$

sind Lösungen der Gleichung (3.3.1).

Nach Satz 3.2.5 sind somit auch $\operatorname{Re}\left(y_{1}^{\star}(x)\right), \operatorname{Im}\left(y_{1}^{\star}(x)\right), \operatorname{Re}\left(y_{2}^{\star}(x)\right)$ und $\operatorname{Im}\left(y_{2}^{\star}(x)\right)$ Lösungen der Gleichung (3.3.1).

Wir wählen (z.B.)

$$
y_{1}(x):=\operatorname{Re}\left(y_{1}^{\star}(x)\right)=\mathrm{e}^{\alpha x} \cdot \cos (\beta x)
$$

und

$$
y_{2}(x):=\operatorname{Im}\left(y_{1}^{\star}(x)\right)=\mathrm{e}^{\alpha x} \cdot \sin (\beta x) .
$$

Dann bilden die Funktionen $y_{1}, y_{2}: \mathbb{R} \rightarrow \mathbb{R}$ ein Fundamentalsystem von (3.3.1) auf $\mathbb{R}$. In der Tat gilt für alle $x \in \mathbb{R}$ :

$$
\begin{gathered}
W(x)=\left|\begin{array}{ll}
y_{1}(x) & y_{2}(x) \\
y_{1}^{\prime}(x) & y_{2}^{\prime}(x)
\end{array}\right| \\
=\left|\begin{array}{cc}
\mathrm{e}^{\alpha x} \cdot \cos (\beta x) & \mathrm{e}^{\alpha x} \cdot \sin (\beta x) \\
\alpha \cdot \mathrm{e}^{\alpha x} \cdot \cos (\beta x)-\beta \cdot \mathrm{e}^{\alpha x} \cdot \sin (\beta x) & \alpha \cdot \mathrm{e}^{\alpha x} \cdot \sin (\beta x)+\beta \cdot \mathrm{e}^{\alpha x} \cdot \cos (\beta x)
\end{array}\right| \\
=\mathrm{e}^{2 \alpha x} \cdot\left(\begin{array}{c}
\alpha \cdot \cos (\beta x) \cdot \sin (\beta x)+\beta \cdot \cos ^{2}(\beta x) \\
\left.\quad-\alpha \cdot \cos (\beta x) \cdot \sin (\beta x)+\beta \cdot \sin ^{2}(\beta x)\right)
\end{array}\right. \\
=\mathrm{e}^{2 \alpha x} \cdot \beta \cdot \underbrace{\left(\cos ^{2}(\beta x)+\sin ^{2}(\beta x)\right)}_{=1}=\beta \cdot \mathrm{e}^{2 \alpha x} \neq 0,
\end{gathered}
$$

denn $\mathrm{e}^{2 \alpha x} \neq 0$ und $\beta \neq 0$ (s.o.). 


\section{Folgerung 3.3.7}

Nach Satz 3.2.11 ist im Fall $\left(\frac{p}{2}\right)^{2}-q<0$ die Funktion $y: \mathbb{R} \rightarrow \mathbb{R}$ mit

$$
\begin{aligned}
y(x) & :=c_{1} \cdot \mathrm{e}^{\alpha x} \cdot \cos (\beta x)+c_{2} \cdot \mathrm{e}^{\alpha x} \cdot \sin (\beta x) \\
& =\mathrm{e}^{\alpha x} \cdot\left(c_{1} \cdot \cos (\beta x)+c_{2} \cdot \sin (\beta x)\right)
\end{aligned}
$$

und beliebigen $c_{1}, c_{2} \in \mathbb{R}$ die allgemeine Lösung von (3.3.1) auf $\mathbb{R}$.

\section{Beispiel 3.3.8}

i)

$$
y^{\prime \prime}(x)+9 y(x)=0, \quad x \in \mathbb{R} .
$$

Die entsprechende charakteristische Gleichung lautet

$$
\lambda^{2}+9=0 \quad \Leftrightarrow \quad \lambda= \pm \mathrm{i} \cdot 3 .
$$

Es gilt $\alpha=0$ und $\beta=3$. Dann bilden die Funktionen $y_{1}, y_{2}: \mathbb{R} \rightarrow \mathbb{R}$ mit

$$
y_{1}(x):=\cos (3 x) \quad \text { und } \quad y_{2}(x):=\sin (3 x)
$$

ein Fundamentalsystem der Gleichung $y^{\prime \prime}(x)+9 y(x)=0$ auf $\mathbb{R}$.

Nach Folgerung 3.3.7 ist

$$
y(x)=c_{1} \cdot \cos (3 x)+c_{2} \cdot \sin (3 x)
$$

mit beliebigen $c_{1}, c_{2} \in \mathbb{R}$ die allgemeine Lösung dieser Gleichung auf $\mathbb{R}$.

ii)

$$
y^{\prime \prime}(x)+2 y^{\prime}(x)+5 y(x)=0, \quad x \in \mathbb{R} .
$$

Die entsprechende charakteristische Gleichung lautet

$$
\lambda^{2}+2 \lambda+5=0 \quad \Leftrightarrow \quad \lambda=-1 \pm \mathrm{i} \cdot 2 .
$$

Es gilt $\alpha=-1$ und $\beta=2$. Dann bilden die Funktionen $y_{1}, y_{2}: \mathbb{R} \rightarrow \mathbb{R}$ mit

$$
y_{1}(x):=\mathrm{e}^{-x} \cdot \cos (2 x) \quad \text { und } \quad y_{2}(x):=\mathrm{e}^{-x} \cdot \sin (2 x)
$$

ein Fundamentalsystem der Gleichung $y^{\prime \prime}(x)+2 y^{\prime}(x)+5 y(x)=0$ auf $\mathbb{R}$. Nach Folgerung 3.3.7 ist

$$
y(x)=\mathrm{e}^{-x} \cdot\left(c_{1} \cdot \cos (2 x)+c_{2} \cdot \sin (2 x)\right)
$$

mit beliebigen $c_{1}, c_{2} \in \mathbb{R}$ die allgemeine Lösung dieser Gleichung auf $\mathbb{R}$. 


\subsection{Gedämpfter elektrischer Schwingkreis}

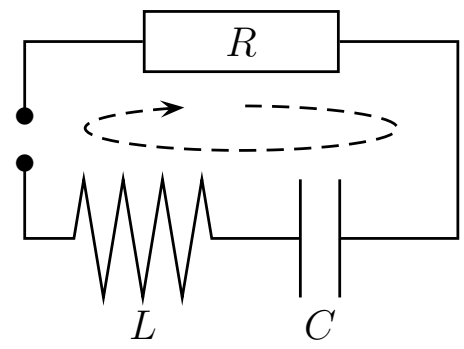

Ein elektrischer Schwingkreis besteht aus einem Oнм'schen Widerstand ${ }^{5} R \geqslant 0$, einem Kondensator mit der Kapazität $C>0$ und einer Spule mit der Induktivität $L>0$. Zum Zeitpunkt $t_{0}=0$ wird der Kondensator geladen und anschließend über den Widerstand und die Spule entladen. Es ergibt sich eine gedämpfte Schwingung.

Nach dem zweiten KIRchноFF'schen Gesetz ${ }^{6}$ gilt:

$$
U_{R}(t)+U_{C}(t)+U_{L}(t)=0, \quad t \in \mathbb{R}^{\geqslant 0} .
$$

Wobei wir mit $U_{R}$ die Spannung am Widerstand, mit $U_{C}$ die Spannung am Kondensator und mit $U_{L}$ die Spannung an der Spule bezeichnen. Es gilt:

$$
U_{R}(t)=R \cdot I(t) ; \quad U_{C}(t)=\frac{Q(t)}{C} ; \quad U_{L}(t)=L \cdot \dot{I}(t) ; \quad I(t)=\dot{Q}(t) ;
$$

dabei sind $I:=I(t)$ Stromstärke im Schwingkreis und $Q:=Q(t)$ Ladung des Kondensators zum Zeitpunkt $t \in \mathbb{R}^{\geqslant 0}$. Dann gilt:

$$
\begin{gathered}
U_{R}(t)+U_{C}(t)+U_{L}(t)=0 \quad \Leftrightarrow \quad R \cdot \dot{Q}(t)+\frac{Q(t)}{C}+L \cdot \ddot{Q}(t)=0 \\
\Leftrightarrow \quad \ddot{Q}(t)+\frac{R}{L} \cdot \dot{Q}(t)+\frac{1}{L \cdot C} \cdot Q(t)=0,
\end{gathered}
$$

d.h., die Ladung am Kondensator genügt einer linearen homogenen Differentialgleichung 2. Ordnung mit konstanten Koeffizienten auf $\mathbb{R}^{\geqslant 0}$. Betrachten wir nun die folgenden Anfangsbedingungen:

$$
Q(0)=Q_{0} \quad \text { und } \quad I(0)=\dot{Q}(0)=0 .
$$

Die charakteristische Gleichung von (3.4.2) lautet:

$$
\lambda^{2}+\frac{R}{L} \cdot \lambda+\frac{1}{L \cdot C}=0,
$$

\footnotetext{
${ }^{5}$ Georg Simon Ohm, 1789-1854

${ }^{6}$ Gustav Robert Kirchhoff, 1824-1887
} 
und die Diskriminante der charakteristischen Gleichung beträgt

$$
D=\left(\frac{R}{2 L}\right)^{2}-\frac{1}{L \cdot C}=\frac{R^{2} \cdot C-4 L}{4 L^{2} \cdot C}
$$

I. Fall $D>0 \quad \Leftrightarrow \quad R^{2} \cdot C-4 L>0$

Dann gilt:

$$
\lambda^{2}+\frac{R}{L} \cdot \lambda+\frac{1}{L \cdot C}=0 \quad \overleftrightarrow{D>0} \quad \lambda=-\frac{R}{2 L} \pm \sqrt{D}
$$

Seien $\lambda_{1}:=-\frac{R}{2 L}-\sqrt{D}$ und $\lambda_{2}:=-\frac{R}{2 L}+\sqrt{D}$.

Dann sind $\left\{\lambda_{1}, \lambda_{2}\right\}$ zwei verschiedene reelle Lösungen der charakteristischen Gleichung. Beachte, dass die beiden Zahlen $\lambda_{1}, \lambda_{2} \in \mathbb{R}$ negativ sind! Nach Folgerung 3.3.3 ist

$$
Q(t)=c_{1} \cdot \mathrm{e}^{\lambda_{1} t}+c_{2} \cdot \mathrm{e}^{\lambda_{2} t}
$$

mit beliebigen $c_{1}, c_{2} \in \mathbb{R}$ die allgemeine Lösung der Gleichung (3.4.2) auf $\mathbb{R}^{\geqslant 0}$.

Für die Ableitung gilt:

$$
I(t)=\dot{Q}(t)=c_{1} \cdot \lambda_{1} \cdot \mathrm{e}^{\lambda_{1} t}+c_{2} \cdot \lambda_{2} \cdot \mathrm{e}^{\lambda_{2} t} .
$$

Mit den Anfangsbedingungen $Q(0)=Q_{0}$ und $\dot{Q}(0)=0$ erhalten wir das lineare Gleichungssystem (mit zwei Unbekannten $c_{1}, c_{2}$ ):

$$
\left\{\begin{array} { l } 
{ c _ { 1 } + c _ { 2 } = Q _ { 0 } } \\
{ c _ { 1 } \cdot \lambda _ { 1 } + c _ { 2 } \cdot \lambda _ { 2 } = 0 }
\end{array} \underset { \lambda _ { 1 } \neq \lambda _ { 2 } } { \Longleftrightarrow } \left\{\begin{array}{l}
c_{1}=\frac{\lambda_{2} \cdot Q_{0}}{\lambda_{2}-\lambda_{1}} \\
c_{2}=\frac{-\lambda_{1} \cdot Q_{0}}{\lambda_{2}-\lambda_{1}}
\end{array}\right.\right.
$$

Dann gilt auf $\mathbb{R}^{\geqslant 0}$ :

und

$$
Q(t)=\frac{Q_{0}}{\lambda_{2}-\lambda_{1}} \cdot\left(\lambda_{2} \cdot \mathrm{e}^{\lambda_{1} t}-\lambda_{1} \cdot \mathrm{e}^{\lambda_{2} t}\right)
$$

$$
I(t)=\dot{Q}(t)=\frac{\lambda_{1} \cdot \lambda_{2} \cdot Q_{0}}{\lambda_{2}-\lambda_{1}} \cdot\left(\mathrm{e}^{\lambda_{1} t}-\mathrm{e}^{\lambda_{2} t}\right)
$$


Mit $\quad \lambda_{1}=-\frac{R}{2 L}-\sqrt{D}$ und $\lambda_{2}=-\frac{R}{2 L}+\sqrt{D}$ folgt:

$$
\begin{aligned}
Q(t) & =\frac{Q_{0}}{2 \sqrt{D}} \cdot \mathrm{e}^{-\frac{R}{2 L} \cdot t}\left(\left(-\frac{R}{2 L}+\sqrt{D}\right) \cdot \mathrm{e}^{-\sqrt{D} \cdot t}-\left(-\frac{R}{2 L}-\sqrt{D}\right) \cdot \mathrm{e}^{\sqrt{D} \cdot t}\right) \\
& =Q_{0} \cdot \mathrm{e}^{-\frac{R}{2 L} \cdot t} \cdot\left(\left(-\frac{R}{2 L}\right) \cdot \frac{\mathrm{e}^{-\sqrt{D} \cdot t}-\mathrm{e}^{\sqrt{D} \cdot t}}{2 \sqrt{D}}+\frac{1}{2} \cdot\left(\mathrm{e}^{-\sqrt{D} \cdot t}+\mathrm{e}^{\sqrt{D} \cdot t}\right)\right)
\end{aligned}
$$

und

$$
I(t)=\frac{Q_{0}}{L \cdot C} \cdot \mathrm{e}^{-\frac{R}{2 L} \cdot t} \cdot \frac{\mathrm{e}^{-\sqrt{D} \cdot t}-\mathrm{e}^{\sqrt{D} \cdot t}}{2 \sqrt{D}} .
$$
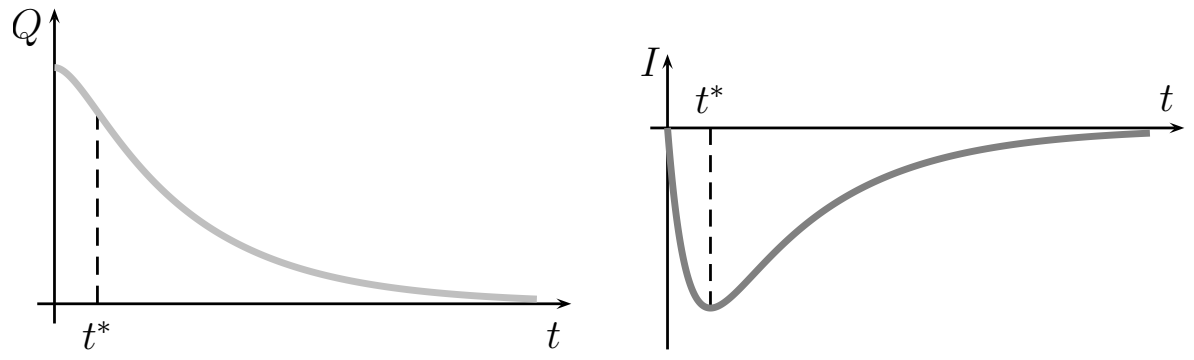

\section{Bemerkung 3.4.1}

i) $\mathrm{Da} I(t)<0$ für alle $t \in \mathbb{R}^{>0}$ und $\lambda_{1}, \lambda_{2}<0$ ist, ist $Q(t) \searrow 0$ für $t \rightarrow+\infty$. Auch ist $I(t) \rightarrow 0$ für $t \rightarrow+\infty$.

ii) Weiter gilt:

$$
\ddot{Q}(t)=\dot{I}(t)=c_{1} \cdot \lambda_{1}^{2} \cdot \mathrm{e}^{\lambda_{1} t}+c_{2} \cdot \lambda_{2}^{2} \cdot \mathrm{e}^{\lambda_{2} t}
$$

mit $c_{1}=\frac{\lambda_{2} \cdot Q_{0}}{\lambda_{2}-\lambda_{1}} \quad$ und $\quad c_{2}=\frac{-\lambda_{1} \cdot Q_{0}}{\lambda_{2}-\lambda_{1}}$, sodass wir bekommen

$$
\ddot{Q}(t)=\frac{\lambda_{1} \cdot \lambda_{2} \cdot Q_{0}}{\lambda_{2}-\lambda_{1}} \cdot\left(\lambda_{1} \cdot \mathrm{e}^{\lambda_{1} t}-\lambda_{2} \cdot \mathrm{e}^{\lambda_{2} t}\right) .
$$

Dann gilt:

$$
\ddot{Q}(t)=0 \quad \Leftrightarrow \quad \lambda_{1} \cdot \mathrm{e}^{\lambda_{1} t}=\lambda_{2} \cdot \mathrm{e}^{\lambda_{2} t} \underset{\lambda_{2} \neq 0}{\Longleftrightarrow} \frac{\lambda_{1}}{\lambda_{2}}=\mathrm{e}^{\left(\lambda_{2}-\lambda_{1}\right) t} .
$$




\section{Übung}

Weisen Sie nach: An der Stelle

$$
t^{*}:=\frac{1}{\lambda_{2}-\lambda_{1}} \cdot \ln \left(\frac{\lambda_{1}}{\lambda_{2}}\right)
$$

hat der Graph der Funktion $Q=Q(t)$ einen Wendepunkt und der Graph der Funktion $I=I(t)$ seinen tiefsten Punkt.

iii) Im Fall $D>0 \Leftrightarrow R^{2} \cdot C-4 L>0$ haben wir keine Schwingung im eigentlichen Sinne. Es liegt ein aperiodisches Verhalten vor.

II. Fall $D=0 \quad \Leftrightarrow \quad R^{2} \cdot C=4 L$

Dann gilt:

$$
\lambda^{2}+\frac{R}{L} \cdot \lambda+\frac{1}{L \cdot C}=0 \quad \stackrel{D=0}{\Longleftrightarrow} \quad \lambda=-\frac{R}{2 L} \quad \text { (mit Vielfachheit } r=2 \text { ). }
$$

Beachte, dass die Zahl $\lambda \in \mathbb{R}$ negativ ist! Nach Folgerung 3.3.5 ist

$$
Q(t)=\mathrm{e}^{\lambda t} \cdot\left(c_{1}+c_{2} \cdot t\right)
$$

mit beliebigen $c_{1}, c_{2} \in \mathbb{R}$ die allgemeine Lösung der Gleichung (3.4.2) auf $\mathbb{R}^{\geqslant 0}$.

Für die Ableitung gilt:

$$
I(t)=\dot{Q}(t)=\lambda \cdot \mathrm{e}^{\lambda t} \cdot\left(c_{1}+c_{2} \cdot t\right)+c_{2} \cdot \mathrm{e}^{\lambda t}
$$

Mit den Anfangsbedingungen $Q(0)=Q_{0}$ und $\dot{Q}(0)=0$ erhalten wir das lineare Gleichungssystem (mit zwei Unbekannten $c_{1}, c_{2}$ ):

$$
\left\{\begin{array} { l } 
{ c _ { 1 } = Q _ { 0 } } \\
{ \lambda \cdot c _ { 1 } + c _ { 2 } = 0 }
\end{array} \Leftrightarrow \left\{\begin{array}{l}
c_{1}=Q_{0} \\
c_{2}=-\lambda \cdot Q_{0}
\end{array}\right.\right.
$$

Dann gilt auf $\mathbb{R}^{\geqslant 0}$ :

$$
Q(t)=Q_{0} \cdot \mathrm{e}^{\lambda t} \cdot(1-\lambda t) \text { und } I(t)=\dot{Q}(t)=-Q_{0} \cdot \lambda^{2} \cdot \mathrm{e}^{\lambda t} \cdot t
$$

Mit $\lambda:=-\frac{R}{2 L}$ folgt:

$$
Q(t)=Q_{0} \cdot \mathrm{e}^{-\frac{R}{2 L} \cdot t} \cdot\left(1+\frac{R}{2 L} \cdot t\right)
$$


und

$$
I(t)=-\left(\frac{R}{2 L}\right)^{2} \cdot Q_{0} \cdot \mathrm{e}^{-\frac{R}{2 L} \cdot t} \cdot t=-\frac{Q_{0}}{L \cdot C} \cdot \mathrm{e}^{-\frac{R}{2 L} \cdot t} \cdot t .
$$

Wobei die letzte Gleichheit daraus folgt, dass

$$
D=0 \quad \Leftrightarrow \quad R^{2} \cdot C=4 L \quad \Leftrightarrow \quad \frac{R^{2}}{4 L^{2}}=\frac{1}{L \cdot C} .
$$
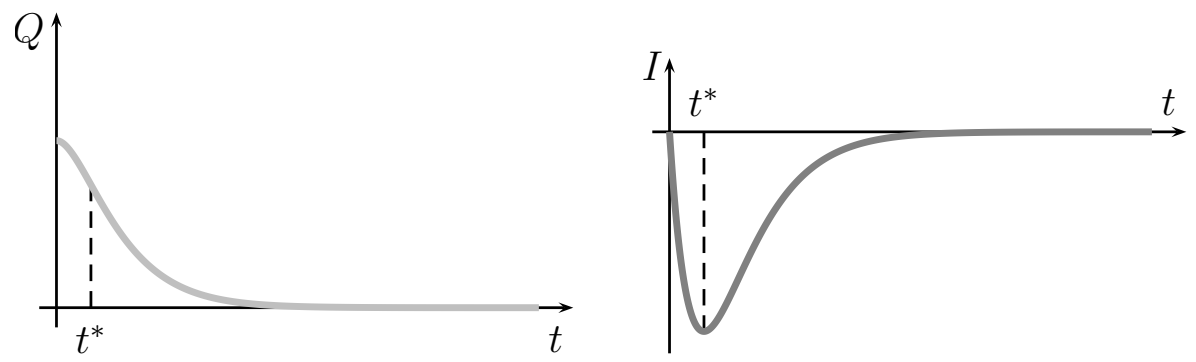

\section{Bemerkung 3.4.2}

i) $\mathrm{Da} I(t)<0$ für alle $t \in \mathbb{R}^{>0}$ und $\lambda<0$ ist, ist $Q(t) \searrow 0$ für $t \rightarrow+\infty$. Auch gilt $I(t) \rightarrow 0$ für $t \rightarrow+\infty$.

ii) Ferner:

$$
\ddot{Q}(t)=\dot{I}(t)=\lambda^{2} \cdot \mathrm{e}^{\lambda t} \cdot\left(c_{1}+c_{2} \cdot t\right)+2 \lambda \cdot \mathrm{e}^{\lambda t} \cdot c_{2} .
$$

Mit $c_{1}=Q_{0}$ und $c_{2}=-\lambda \cdot Q_{0}$ bekommen wir

$$
\ddot{Q}(t)=-\lambda^{2} \cdot Q_{0} \cdot \mathrm{e}^{\lambda t}(\lambda \cdot t+1) .
$$

Dann gilt:

$$
\ddot{Q}(t)=0 \quad \Leftrightarrow \quad \lambda \cdot t+1=0 .
$$

\section{Übung}

Weisen Sie nach: An der Stelle

$$
t^{*}=-\frac{1}{\lambda}=\frac{2 L}{R}
$$

hat der Graph der Funktion $Q=Q(t)$ einen Wendepunkt und der Graph der Funktion $I=I(t)$ seinen tiefsten Punkt. 
iii) Im Fall $D=0 \quad \Leftrightarrow \quad R^{2} \cdot C=4 L$ haben wir keine Schwingung im eigentlichen Sinne. Es liegt ein aperiodisches Verhalten vor.

iv) Für $x \rightarrow 0$ gilt $\mathrm{e}^{x} \approx 1+x$, sodass wir für $D \rightarrow 0$ erhalten:

$$
\frac{\mathrm{e}^{-\sqrt{D} \cdot t}-\mathrm{e}^{\sqrt{D} \cdot t}}{2 \sqrt{D}} \approx(-t) .
$$

Fall I verhält sich somit wie Fall II, wenn $D \rightarrow 0$.

III. Fall $D<0 \quad \Leftrightarrow \quad R^{2} \cdot C-4 L<0$

Dann gilt:

$$
\lambda^{2}+\frac{R}{L} \cdot \lambda+\frac{1}{L \cdot C}=0 \quad \overleftrightarrow{D<0} \quad \lambda=\alpha \pm \mathrm{i} \cdot \beta
$$

wobei $\alpha, \beta \in \mathbb{R}$ mit

$$
\alpha:=-\frac{R}{2 L} \quad \text { und } \quad \beta:=\sqrt{\frac{1}{L \cdot C}-\left(\frac{R}{2 L}\right)^{2}} .
$$

Beachte: $\alpha<0$ und $\beta>0$. Wir erhalten zwei verschiedene komplexe Lösungen der charakteristischen Gleichung. Nach Folgerung 3.3.7 ist

$$
Q(t)=\mathrm{e}^{\alpha t} \cdot\left(c_{1} \cdot \cos (\beta t)+c_{2} \cdot \sin (\beta t)\right)
$$

mit beliebigen $c_{1}, c_{2} \in \mathbb{R}$ die allgemeine Lösung der Gleichung (3.4.2) auf $\mathbb{R}^{\geqslant 0}$. Für die Ableitung gilt:

$$
\begin{aligned}
& I(t)=\dot{Q}(t) \\
& =\mathrm{e}^{\alpha t} \cdot\left(\alpha \cdot c_{1} \cdot \cos (\beta t)+\alpha \cdot c_{2} \cdot \sin (\beta t)\right. \\
& \left.-\beta \cdot c_{1} \cdot \sin (\beta t)+\beta \cdot c_{2} \cdot \cos (\beta t)\right) .
\end{aligned}
$$

Mit den Anfangsbedingungen $Q(0)=Q_{0}$ und $\dot{Q}(0)=0$ erhalten wir das lineare Gleichungssystem (mit zwei Unbekannten $c_{1}, c_{2}$ ):

$$
\left\{\begin{array} { l } 
{ c _ { 1 } = Q _ { 0 } } \\
{ \alpha \cdot c _ { 1 } + \beta \cdot c _ { 2 } = 0 }
\end{array} \quad \overleftrightarrow { \beta > 0 } \quad \left\{\begin{array}{l}
c_{1}=Q_{0} \\
c_{2}=-\frac{\alpha}{\beta} \cdot Q_{0}
\end{array}\right.\right.
$$


Dann gilt auf $\mathbb{R}^{\geqslant 0}$ :

$$
Q(t)=Q_{0} \cdot \mathrm{e}^{-\frac{R}{2 L} \cdot t} \cdot\left(\cos (\beta t)-\frac{\alpha}{\beta} \sin (\beta t)\right)
$$

und

$$
\begin{aligned}
I(t) & =\dot{Q}(t) \\
& =Q_{0} \cdot \mathrm{e}^{-\frac{R}{2 L} \cdot t} \cdot\left(-\frac{\alpha^{2}}{\beta}-\beta\right) \cdot \sin (\beta t) \\
& =-\frac{Q_{0}}{L \cdot C} \cdot \mathrm{e}^{-\frac{R}{2 L} \cdot t} \cdot \frac{\sin (\beta t)}{\beta},
\end{aligned}
$$

wobei wir $\alpha^{2}+\beta^{2}=\frac{1}{L \cdot C}$ ausgenutzt haben.

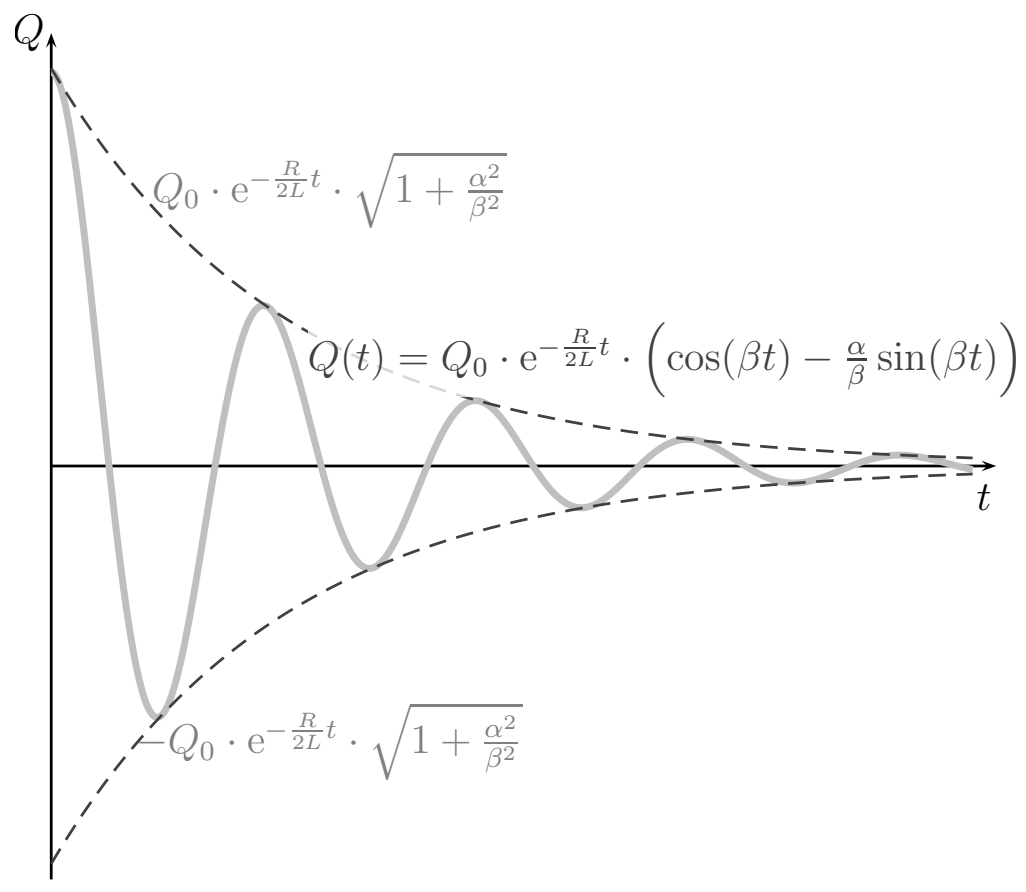




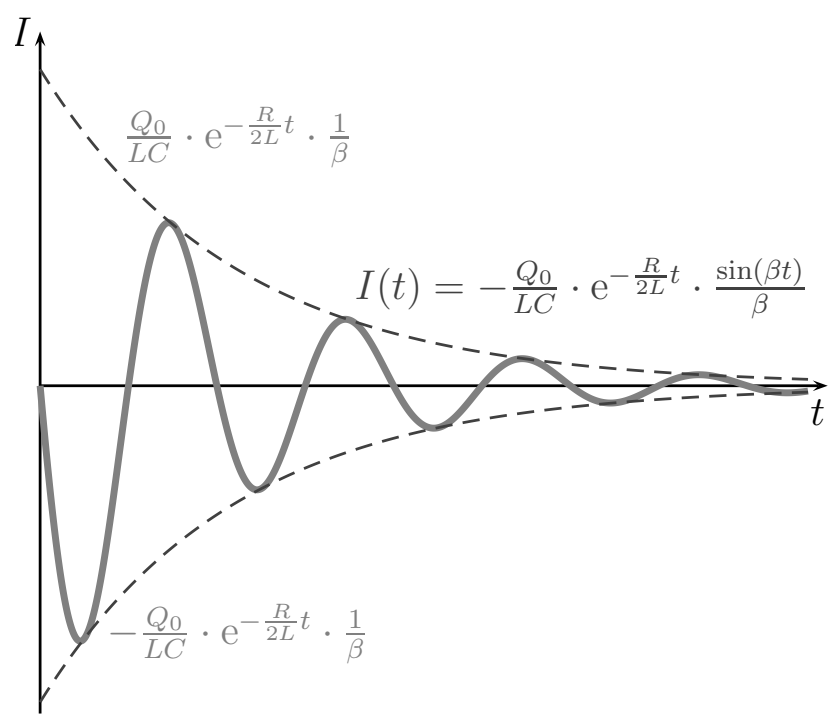

\section{Bemerkung 3.4.3}

i) Es gilt: $Q(t) \rightarrow 0$ für $t \rightarrow+\infty$. Auch ist $I(t) \rightarrow 0$ für $t \rightarrow+\infty$.

ii) Für die elektrische Ladung gilt:

$$
\begin{aligned}
Q(t) & =Q_{0} \cdot \mathrm{e}^{-\frac{R}{2 L} \cdot t} \cdot\left(\cos (\beta t)-\frac{\alpha}{\beta} \cdot \sin (\beta t)\right) \\
& \underset{\beta>0}{=} Q_{0} \cdot \mathrm{e}^{-\frac{R}{2 L} \cdot t} \cdot \sqrt{1+\frac{\alpha^{2}}{\beta^{2}}} \cdot\left(\frac{\beta}{\sqrt{\alpha^{2}+\beta^{2}}} \cdot \cos (\beta t)\right. \\
& \left.=Q_{0} \cdot \mathrm{e}^{-\frac{R}{2 L} \cdot t} \cdot \sqrt{\frac{\alpha}{\sqrt{\alpha^{2}+\beta^{2}}}} \cdot \sin (\beta t)\right)
\end{aligned}
$$

wobei der Winkel $\varphi \in\left(-\frac{\pi}{2} ; 0\right)$ eindeutig festgelegt ist durch

$$
\cos (\varphi):=\frac{\beta}{\sqrt{\alpha^{2}+\beta^{2}}}>0 \text { und } \sin (\varphi):=\frac{\alpha}{\sqrt{\alpha^{2}+\beta^{2}}}<0
$$

iii) Im Fall $D<0 \quad \Leftrightarrow \quad R^{2} \cdot C-4 L<0$ haben wir eine gedämpfte Schwingung (bei schwacher Dämpfung). 
iv) Für $x \rightarrow 0$ gilt $\sin (x) \approx x$ und $\cos (x) \approx 1$ ist, sodass wir für $\beta \rightarrow 0$ erhalten:

$$
\frac{\sin (\beta t)}{\beta} \approx t
$$

und

$$
\left(\cos (\beta t)-\frac{\alpha}{\beta} \cdot \sin (\beta t)\right) \approx(1-\alpha \cdot t)=1+\frac{R}{2 L} \cdot t .
$$

Fall III verhält sich somit wie Fall II, wenn $\beta \rightarrow 0$ ist.

\section{Sonderfall 3.4.4}

Liegt kein Widerstand vor, d.h., haben wir $R=0$, so gilt für den III. Fall:

$$
\alpha=0 \text { und } \beta=\sqrt{\frac{1}{L \cdot C}} .
$$

Aus den Funktionsgleichungen für $Q(t)$ bzw. I $(t)$ des III. Falls bekommen wir:

$$
Q(t)=Q_{0} \cdot \cos (\beta t) \quad \text { und } \quad I(t)=-\beta \cdot Q_{0} \cdot \sin (\beta t)
$$
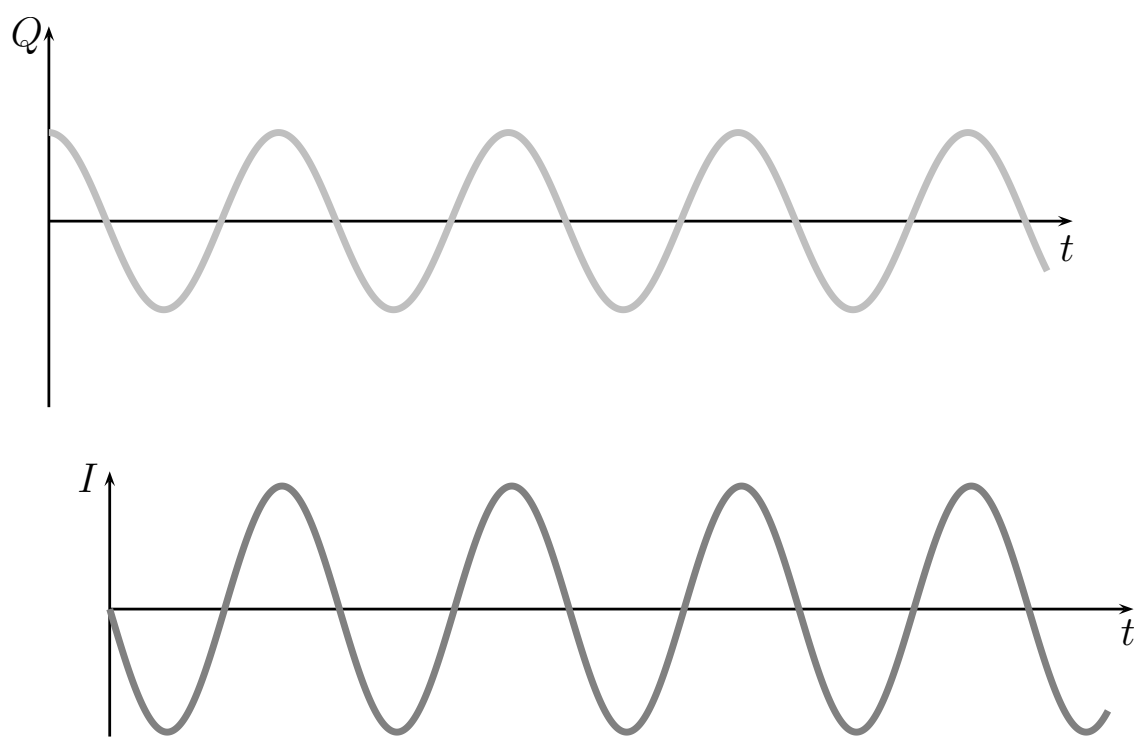

Im Fall $R=0$ haben wir eine ungedämpfte Schwingung:

$$
\ddot{Q}(t)+\frac{1}{L \cdot C} \cdot Q(t)=0
$$

(Mit den Anfangsbedingungen $Q(0)=Q_{0}$ und $\dot{Q}(0)=0$.)

Die entsprechende charakteristische Gleichung lautet:

$$
\lambda^{2}+\frac{1}{L \cdot C}=0
$$




\subsection{Analogien bei mechanischen und elektro- magnetischen gedämpften Schwingungen}

\begin{tabular}{|c|c|}
\hline Mechanische Schwingung & Elektromagnetische Schwingung \\
\hline Koordinate $x$ & Ladung $Q$ \\
\hline Geschwindigkeit $v=\dot{x}$ & Stromstärke $I=\dot{Q}$ \\
\hline Beschleunigung $a=\ddot{x}$ & Zeitliche Änderung der Stromstärke $\dot{I}$ \\
\hline Masse $m$ & Induktivität $L$ \\
\hline Richtgröße $k$ (Federkonstante) & $\frac{1}{C}$, wobei $C:=$ Kapazität \\
\hline Dämpfungskonstante $b$ & Ohmscher Widerstand $R$ \\
\hline $\begin{array}{l}\text { Schwingungsgleichung } \\
m \cdot \ddot{x}(t)+b \cdot \dot{x}(t)+k \cdot x(t)=0\end{array}$ & $\begin{array}{r}\text { Schwingungsgleichung } \\
L \cdot \ddot{Q}(t)+R \cdot \dot{Q}(t)+\frac{Q(t)}{C}=0\end{array}$ \\
\hline $\begin{array}{l}\text { ungedämpfte Kreisfrequenz } \\
\omega_{0}=\sqrt{\frac{k}{m}}\end{array}$ & $\begin{array}{l}\text { ungedämpfte Kreisfrequenz } \\
\qquad \omega_{0}=\sqrt{\frac{1}{L \cdot C}}\end{array}$ \\
\hline $\begin{array}{l}\text { gedämpfte Kreisfrequenz } \\
\omega=\sqrt{\frac{k}{m}-\left(\frac{b}{2 m}\right)^{2}}\end{array}$ & $\begin{array}{l}\text { gedämpfte Kreisfrequenz } \\
\omega=\sqrt{\frac{1}{L \cdot C}-\left(\frac{R}{2 L}\right)^{2}}\end{array}$ \\
\hline
\end{tabular}




\subsection{Lineare inhomogene Differentialgleichung zweiter Ordnung}

\section{Satz 3.6.1}

Seien die Funktionen $p, q, f: I \rightarrow \mathbb{R}$ auf einem Intervall I definiert und stetig. Dann lässt sich die Lösungsmenge $\mathbb{L}$ der inhomogenen Differentialgleichung 2. Ordnung

$$
y^{\prime \prime}(x)+p(x) \cdot y^{\prime}(x)+q(x) \cdot y(x)=f(x)
$$

auf I schreiben als: $\mathbb{L}=y^{*}+\mathbb{L}_{0}$, wobei $\mathbb{L}_{0}$ die Lösungsmenge der entsprechenden homogenen Gleichung

$$
y^{\prime \prime}(x)+p(x) \cdot y^{\prime}(x)+q(x) \cdot y(x)=0
$$

und $y^{*}(x)$ eine (sog. partikuläre) Lösung der inhomogenen Gleichung (3.6.1) ist.

BEWEIS:

Wir haben bereits in Satz 3.2.15 gesehen, dass $\mathbb{L}_{0}$ nichtleer ist. Wir werden in Bemerkung 3.6.4 zeigen, dass auch $\mathbb{L} \neq \varnothing$, mit anderen Worten existiert eine partikuläre Lösung $y^{*}(x)$ von (3.6.1) und es gilt auf $I$ :

$$
\left(y^{*}\right)^{\prime \prime}(x)+p(x) \cdot\left(y^{*}\right)^{\prime}(x)+q(x) \cdot y^{*}(x)=f(x) .
$$

1. Sei $y_{L}(x) \in \mathbb{L}$ eine beliebige Lösung von (3.6.1). Dann

$$
y_{L}^{\prime \prime}(x)+p(x) \cdot y_{L}^{\prime}(x)+q(x) \cdot y_{L}(x)=f(x)
$$

auf $I$ und somit

$$
\begin{array}{r}
y_{L}^{\prime \prime}(x)-\left(y^{*}\right)^{\prime \prime}(x)+p(x) \cdot\left(y_{L}^{\prime}(x)-\left(y^{*}\right)^{\prime}(x)\right)+q(x) \cdot\left(y_{L}(x)-y^{*}(x)\right)=0 \\
\Leftrightarrow\left(y_{L}(x)-y^{*}(x)\right)^{\prime \prime}+p(x) \cdot\left(y_{L}(x)-y^{*}(x)\right)^{\prime}+q(x) \cdot\left(y_{L}(x)-y^{*}(x)\right)=0
\end{array}
$$

D.h., $\widetilde{y}(x):=y_{L}(x)-y^{*}(x)$ ist eine Lösung der homogenen Gleichung

$$
y^{\prime \prime}(x)+p(x) \cdot y^{\prime}(x)+q(x) \cdot y(x)=0
$$

auf $I$. Also gilt für jedes $y_{L}(x) \in \mathbb{L}$ :

$$
y_{L}(x)=y^{*}(x)+\widetilde{y}(x) \in y^{*}(x)+\mathbb{L}_{0} \quad \text { und } \quad \mathbb{L} \subseteq y^{*}(x)+\mathbb{L}_{0} .
$$


2. Sei nun $\widetilde{y}(x) \in \mathbb{L}_{0}$ eine beliebige Lösung der homogenen Gleichung. Dann

$$
\widetilde{y}^{\prime \prime}(x)+p(x) \cdot \widetilde{y}^{\prime}(x)+q(x) \cdot \widetilde{y}(x)=0
$$

auf $I$. Da $y^{*}(x)$ eine ( partikuläre) Lösung der inhomogenen Gleichung (3.6.1) ist, folgt:

$$
\begin{aligned}
& \widetilde{y}^{\prime \prime}(x)+\left(y^{*}\right)^{\prime \prime}(x)+p(x) \cdot\left(\widetilde{y}^{\prime}(x)+\left(y^{*}\right)^{\prime}(x)\right)+q(x) \cdot\left(\widetilde{y}(x)+y^{*}(x)\right)=f(x) \\
& \Leftrightarrow\left(\widetilde{y}(x)+y^{*}(x)\right)^{\prime \prime}+p(x) \cdot\left(\widetilde{y}(x)+y^{*}(x)\right)^{\prime}+q(x) \cdot\left(\widetilde{y}(x)+y^{*}(x)\right)=f(x)
\end{aligned}
$$

D.h., $y(x):=\widetilde{y}(x)+y^{*}(x)$ ist eine Lösung der inhomogenen Gleichung (3.6.1) auf $I$. Also gilt für jedes $\widetilde{y}(x) \in \mathbb{L}_{0}$ :

$$
y^{*}(x)+\widetilde{y}(x) \in \mathbb{L} \quad \text { und } \quad y^{*}(x)+\mathbb{L}_{0} \subseteq \mathbb{L} .
$$

Insgesamt haben wir $\mathbb{L}=y^{*}(x)+\mathbb{L}_{0}$ gezeigt.

\section{Folgerung 3.6.2}

Sei $\left\{y_{1}, y_{2}\right\}$ ein Fundamentalsystem der homogenen Differentialgleichung

$$
y^{\prime \prime}(x)+p(x) \cdot y^{\prime}(x)+q(x) \cdot y(x)=0 \quad \text { auf } I .
$$

Lässt man in $c_{1} \cdot y_{1}(x)+c_{2} \cdot y_{2}(x)$ die Koeffizienten $c_{1}, c_{2} \in \mathbb{R}$ unabhängig voneinander die Menge der reellen Zahlen durchlaufen, so erhält man alle Lösungen der homogenen Gleichung (3.6.1h).

Also gilt für die Lösungsmenge

$$
\mathbb{L}_{0}=\left\{c_{1} \cdot y_{1}(x)+c_{2} \cdot y_{2}(x): c_{1}, c_{2} \in \mathbb{R}\right\}
$$

dabei heißt $c_{1} \cdot y_{1}(x)+c_{2} \cdot y_{2}(x)$ allgemeine Lösung der homogenen Gleichung. Ist jetzt $y^{*}(x)$ eine partikuläre Lösung der inhomogenen Gleichung (3.6.1), dann gilt nach Satz 3.6.1 für die Lösungsmenge:

$$
\mathbb{L}=\left\{c_{1} \cdot y_{1}(x)+c_{2} \cdot y_{2}(x)+y^{*}(x): c_{1}, c_{2} \in \mathbb{R}\right\} .
$$

Man sagt: Durch $y(x)=c_{1} \cdot y_{1}(x)+c_{2} \cdot y_{2}(x)+y^{*}(x)$ ist die allgemeine Lösung der linearen inhomogenen Differentialgleichung 2. Ordnung (3.6.1) gegeben. 
Oder auch: Die allgemeine Lösung der linearen inhomogenen Differentialgleichung 2. Ordnung hat die Form

$$
y(x)=\widetilde{y}(x)+y^{*}(x),
$$

wobei $\widetilde{y}(x)$ die allgemeine Lösung der entsprechenden linearen homogenen Gleichung und $y^{*}(x)$ eine partikuläre Lösung der inhomogenen Differentialgleichung 2. Ordnung (3.6.1) ist.

\section{Beispiel 3.6.3}

$$
y^{\prime \prime}(x)-9 y(x)=x, \quad x \in \mathbb{R} .
$$

1) Die entsprechende homogene Differentialgleichung lautet

$$
y^{\prime \prime}(x)-9 y(x)=0
$$

und die charakteristische Gleichung ist

$$
\lambda^{2}-9=0 \quad \Leftrightarrow \quad \lambda= \pm 3 .
$$

Nach Folgerung 3.3.3 ist

$$
\widetilde{y}(x):=c_{1} \cdot \mathrm{e}^{3 x}+c_{2} \cdot \mathrm{e}^{-3 x} \quad \text { mit beliebigen } \quad c_{1}, c_{2} \in \mathbb{R}
$$

die allgemeine Lösung der entsprechenden homogenen Gleichung (3.6.3h).

2) Wie ist nun $y^{*}(x)$ zu wählen?

Dazu betrachten wir den Ansatz $y^{*}(x):=A \cdot x+B$ mit $A, B \in \mathbb{R}$. Dann gilt für jedes $x \in \mathbb{R}$ :

$$
\begin{gathered}
\left(y^{*}\right)^{\prime}(x)=A, \quad\left(y^{*}\right)^{\prime \prime}(x)=0 \quad \text { und } \\
\left(y^{*}\right)^{\prime \prime}(x)-9 y^{*}(x)=x \quad \Leftrightarrow \quad-9 \cdot(A \cdot x+B)=x .
\end{gathered}
$$

Ein Koeffizientenvergleich liefert:

$$
\left\{\begin{array} { r l } 
{ - 9 A } & { = 1 } \\
{ B } & { = 0 }
\end{array} \quad \Leftrightarrow \quad \left\{\begin{array}{l}
A=-\frac{1}{9} \\
B=0
\end{array}\right.\right.
$$


Dann ist $y^{*}(x):=-\frac{1}{9} \cdot x$ eine partikuläre Lösung der inhomogenen Gleichung (3.6.3). Nach Satz 3.6.1 und Folgerung 3.6.2 ist

$$
y(x)=\widetilde{y}(x)+y^{*}(x)=c_{1} \cdot \mathrm{e}^{3 x}+c_{2} \cdot \mathrm{e}^{-3 x}-\frac{1}{9} \cdot x
$$

die allgemeine Lösung der inhomogenen Differentialgleichung (3.6.3) auf $\mathbb{R}$.

\section{Bemerkung 3.6.4 (Variation der Konstanten)}

Seien die Funktionen $p, q, f: I \rightarrow \mathbb{R}$ auf einem Intervall I definiert und stetig. Sei weiter $\left\{y_{1}, y_{2}\right\}$ ein Fundamentalsystem der homogenen Gleichung

$$
y^{\prime \prime}(x)+p(x) \cdot y^{\prime}(x)+q(x) \cdot y(x)=0
$$

auf I. Eine partikuläre Lösung der inhomogenen Differentialgleichung

$$
y^{\prime \prime}(x)+p(x) \cdot y^{\prime}(x)+q(x) \cdot y(x)=f(x)
$$

findet man nach dem Verfahren der Variation der Konstanten:

Wir suchen eine partikuläre Lösung in der Form:

$$
y^{*}(x)=c_{1}(x) \cdot y_{1}(x)+c_{2}(x) \cdot y_{2}(x),
$$

wobei $c_{1}, c_{2}: I \rightarrow \mathbb{R}$ unbekannte Funktionen sind. Dann gilt (mit Voraussetzung, dass die Funktionen $c_{1}, c_{2}$ in I differenzierbar sind):

$$
\begin{aligned}
\left(y^{*}\right)^{\prime}(x) & =c_{1}^{\prime}(x) \cdot y_{1}(x)+c_{1}(x) \cdot y_{1}^{\prime}(x)+c_{2}^{\prime}(x) \cdot y_{2}(x)+c_{2}(x) \cdot y_{2}^{\prime}(x) \\
& =c_{1}(x) \cdot y_{1}^{\prime}(x)+c_{2}(x) \cdot y_{2}^{\prime}(x)+c_{1}^{\prime}(x) \cdot y_{1}(x)+c_{2}^{\prime}(x) \cdot y_{2}(x) .
\end{aligned}
$$

Als zusätzliche Bedingung nehmen wir an:

$$
c_{1}^{\prime}(x) \cdot y_{1}(x)+c_{2}^{\prime}(x) \cdot y_{2}(x)=0 \quad \text { auf } I .
$$

Somit erhalten wir

$$
\left(y^{*}\right)^{\prime}(x)=c_{1}(x) \cdot y_{1}^{\prime}(x)+c_{2}(x) \cdot y_{2}^{\prime}(x)
$$

und folglich

$$
\left(y^{*}\right)^{\prime \prime}(x)=c_{1}^{\prime}(x) \cdot y_{1}^{\prime}(x)+c_{1}(x) \cdot y_{1}^{\prime \prime}(x)+c_{2}^{\prime}(x) \cdot y_{2}^{\prime}(x)+c_{2}(x) \cdot y_{2}^{\prime \prime}(x) .
$$


In die Differentialgleichung (3.6.1) eingesetzt ergibt das:

$$
\begin{aligned}
& {\left[c_{1}^{\prime}(x) \cdot y_{1}^{\prime}(x)+c_{1}(x) \cdot y_{1}^{\prime \prime}(x)+c_{2}^{\prime}(x) \cdot y_{2}^{\prime}(x)+c_{2}(x) \cdot y_{2}^{\prime \prime}(x)\right]} \\
& +p(x) \cdot\left[c_{1}(x) \cdot y_{1}^{\prime}(x)+c_{2}(x) \cdot y_{2}^{\prime}(x)\right] \\
& +q(x) \cdot\left[c_{1}(x) \cdot y_{1}(x)+c_{2}(x) \cdot y_{2}(x)\right]=f(x) \\
& \Longleftrightarrow \quad c_{1}(x) \cdot(\underbrace{y_{1}^{\prime \prime}(x)+p(x) \cdot y_{1}^{\prime}(x)+q(x) \cdot y_{1}(x)}_{=0}) \\
& +c_{2}(x) \cdot(\underbrace{y_{2}^{\prime \prime}(x)+p(x) \cdot y_{2}^{\prime}(x)+q(x) \cdot y_{2}(x)}_{=0}) \\
& +\left(c_{1}^{\prime}(x) \cdot y_{1}^{\prime}(x)+c_{2}^{\prime}(x) \cdot y_{2}^{\prime}(x)\right)=f(x),
\end{aligned}
$$

da die Funktionen $y_{1}, y_{2}$ Lösungen der homogenen Differentialgleichung sind, folgt

$$
c_{1}^{\prime}(x) \cdot y_{1}^{\prime}(x)+c_{2}^{\prime}(x) \cdot y_{2}^{\prime}(x)=f(x)
$$

Mit der zusätzlich angenommenen Bedingung $c_{1}^{\prime}(x) \cdot y_{1}(x)+c_{2}^{\prime}(x) \cdot y_{2}(x)=0$ erhalten wir ein lineares Gleichungssystem für die Unbekannten $c_{1}^{\prime}(x), c_{2}^{\prime}(x)$ :

$$
\left\{\begin{array}{l}
c_{1}^{\prime}(x) \cdot y_{1}(x)+c_{2}^{\prime}(x) \cdot y_{2}(x)=0 \\
c_{1}^{\prime}(x) \cdot y_{1}^{\prime}(x)+c_{2}^{\prime}(x) \cdot y_{2}^{\prime}(x)=f(x)
\end{array}\right.
$$

mit der Hauptdeterminante

$$
\triangle:=\left|\begin{array}{ll}
y_{1}(x) & y_{2}(x) \\
y_{1}^{\prime}(x) & y_{2}^{\prime}(x)
\end{array}\right|
$$

Nach Definition 3.2.7 ist $\triangle=W(x)$ die WRONSKI-Determinante der Funktionen $y_{1}, y_{2}$. Da $\left\{y_{1}, y_{2}\right\}$ ein Fundamentalsystem der homogenen Differentialgleichung ist, gilt $W(x) \neq 0$ auf ganz I. Dann ist das lineare Gleichungssystem mit den Unbekannten $c_{1}^{\prime}(x), c_{2}^{\prime}(x)$ eindeutig lösbar. Mit der CRAMER'schen Regel ${ }^{7}$ erhalten wir

$$
\triangle_{1}:=\left|\begin{array}{cc}
0 & y_{2}(x) \\
f(x) & y_{2}^{\prime}(x)
\end{array}\right|, \quad \Delta_{2}:=\left|\begin{array}{cc}
y_{1}(x) & 0 \\
y_{1}^{\prime}(x) & f(x)
\end{array}\right|
$$

${ }^{7}$ Gabriel Cramer, 1704-1752 
und

$$
c_{1}^{\prime}(x)=\frac{\triangle_{1}}{\triangle}=-\frac{y_{2}(x) \cdot f(x)}{W(x)}, \quad c_{2}^{\prime}(x)=\frac{\triangle_{2}}{\triangle}=\frac{y_{1}(x) \cdot f(x)}{W(x)} .
$$

Da die Funktionen $\frac{y_{1} \cdot f}{W}, \frac{y_{2} \cdot f}{W}: I \rightarrow \mathbb{R}$ auf I stetig sind, besitzen sie dort ihre Stammfunktionen. Wir erhalten die Funktionen $c_{1}, c_{2}: I \rightarrow \mathbb{R}$ und somit eine partikuläre Lösung der inhomogenen Differentialgleichung in der Form

$$
y^{*}(x)=c_{1}(x) \cdot y_{1}(x)+c_{2}(x) \cdot y_{2}(x) .
$$

Beispiel 3.6.5

$$
y^{\prime \prime}(x)+y(x)=\frac{1}{\sin (x)}, \quad x \in(0 ; \pi) .
$$

1) Die entsprechende homogene Differentialgleichung lautet

$$
y^{\prime \prime}(x)+y(x)=0
$$

und die charakteristische Gleichung ist

$$
\lambda^{2}+1=0 \quad \Leftrightarrow \quad \lambda= \pm \mathrm{i} .
$$

D.h., $\alpha=0$ und $\beta=1$. Nach Folgerung 3.3.7 ist

$$
\widetilde{y}(x):=c_{1} \cdot \cos (x)+c_{2} \cdot \sin (x) \quad \text { mit beliebigen } \quad c_{1}, c_{2} \in \mathbb{R}
$$

die allgemeine Lösung der entsprechenden homogenen Differentialgleichung (3.6.5h) auf $(0 ; \pi)$. Die Funktionen $y_{1}(x):=\cos (x)$ und $y_{2}(x):=\sin (x)$ bilden ein Fundamentalsystem der homogenen Differentialgleichung auf $(0 ; \pi)$.

2) Wir suchen nun $y^{*}(x)$ mit dem Verfahren der Variation der Konstanten. Nach Bemerkung 3.6.4 erhalten wir auf dem offenen Intervall $(0 ; \pi)$ :

$$
\left\{\begin{aligned}
c_{1}^{\prime}(x) \cdot \cos (x)+c_{2}^{\prime}(x) \cdot \sin (x) & =0 \\
c_{1}^{\prime}(x) \cdot(-\sin (x))+c_{2}^{\prime}(x) \cdot \cos (x) & =\frac{1}{\sin (x)} .
\end{aligned}\right.
$$

mit der Hauptdeterminante

$$
\triangle:=W(x)=\left|\begin{array}{ll}
y_{1}(x) & y_{2}(x) \\
y_{1}^{\prime}(x) & y_{2}^{\prime}(x)
\end{array}\right|=\left|\begin{array}{cc}
\cos (x) & \sin (x) \\
-\sin (x) & \cos (x)
\end{array}\right|=\cos ^{2}(x)+\sin ^{2}(x)=1
$$


und

$$
\triangle_{1}:=\left|\begin{array}{cc}
0 & \sin (x) \\
\frac{1}{\sin (x)} & \cos (x)
\end{array}\right|=-1, \quad \Delta_{2}:=\left|\begin{array}{cc}
\cos (x) & 0 \\
-\sin (x) & \frac{1}{\sin (x)}
\end{array}\right|=\frac{\cos (x)}{\sin (x)}
$$

Nach der CRAmer'schen Regel gilt:

$$
c_{1}^{\prime}(x)=\frac{\triangle_{1}}{\triangle}=-1 \quad \text { und } \quad c_{2}^{\prime}(x)=\frac{\triangle_{2}}{\triangle}=\frac{\cos (x)}{\sin (x)}
$$

und somit (beachte, dass $\sin (x)>0$ auf $(0 ; \pi)$ ist):

$c_{1}(x)=-x+m \quad$ und $\quad c_{2}(x)=\ln (\sin (x))+n \quad$ mit beliebigen $\quad m, n \in \mathbb{R}$.

Mit $m=0$ und $n=0$ bekommen wir folgende partikuläre Lösung der inhomogenen Differentialgleichung

$$
y^{*}(x)=c_{1}(x) \cdot y_{1}(x)+c_{2}(x) \cdot y_{2}(x)=-x \cdot \cos (x)+\sin (x) \cdot \ln (\sin (x)) .
$$

Die allgemeine Lösung der inhomogenen Differentialgleichung (3.6.5) auf $(0 ; \pi)$ ist somit

$$
\begin{aligned}
y(x) & =\widetilde{y}(x)+y^{*}(x) \\
& =c_{1} \cdot \cos (x)+c_{2} \cdot \sin (x)-x \cdot \cos (x)+\sin (x) \cdot \ln (\sin (x))
\end{aligned}
$$

mit beliebigen $c_{1}, c_{2} \in \mathbb{R}$.

\section{Satz 3.6.6 (Superpositionsprinzip)}

Seien die Funktionen $p, q, f_{1}, f_{2}: I \rightarrow \mathbb{R}$ auf einem Intervall I definiert und stetig. Seien weiter $y_{1}^{*}(x)$ eine partikuläre Lösung von

$$
y^{\prime \prime}(x)+p(x) \cdot y^{\prime}(x)+q(x) \cdot y(x)=f_{1}(x)
$$

und $y_{2}^{*}(x)$ eine partikuläre Lösung von

$$
y^{\prime \prime}(x)+p(x) \cdot y^{\prime}(x)+q(x) \cdot y(x)=f_{2}(x) .
$$

Dann ist $y^{*}(x):=y_{1}^{*}(x)+y_{2}^{*}(x)$ eine partikuläre Lösung von

$$
y^{\prime \prime}(x)+p(x) \cdot y^{\prime}(x)+q(x) \cdot y(x)=f_{1}(x)+f_{2}(x) .
$$


BEWEIS:

Einsetzen von $y^{*}(x)$ in (3.6.7) liefert die Behauptung, weil $y_{1}^{*}(x)$ und $y_{2}^{*}(x)$ partikuläre Lösungen der entsprechenden Gleichungen sind. In der Tat gilt für jedes $x \in I$ :

$$
\left(y^{*}\right)^{\prime}(x)=\left(y_{1}^{*}\right)^{\prime}(x)+\left(y_{2}^{*}\right)^{\prime}(x), \quad\left(y^{*}\right)^{\prime \prime}(x)=\left(y_{1}^{*}\right)^{\prime \prime}(x)+\left(y_{2}^{*}\right)^{\prime \prime}(x)
$$

und

$$
\begin{aligned}
& \left(y^{*}\right)^{\prime \prime}(x)+p(x) \cdot\left(y^{*}\right)^{\prime}(x)+q(x) \cdot y^{*}(x) \\
& =\left(y_{1}^{*}\right)^{\prime \prime}(x)+\left(y_{2}^{*}\right)^{\prime \prime}(x)+p(x) \cdot\left(\left(y_{1}^{*}\right)^{\prime}(x)+\left(y_{2}^{*}\right)^{\prime}(x)\right) \\
& +q(x) \cdot\left(y_{1}^{*}(x)+y_{2}^{*}(x)\right) \\
& =\left(y_{1}^{*}\right)^{\prime \prime}(x)+p(x) \cdot\left(y_{1}^{*}\right)^{\prime}(x)+q(x) \cdot y_{1}^{*}(x) \\
& +\left(y_{2}^{*}\right)^{\prime \prime}(x)+p(x) \cdot\left(y_{2}^{*}\right)^{\prime}(x)+q(x) \cdot y_{2}^{*}(x) \\
& \underset{(3.6 .6 \mathrm{~b})}{\stackrel{(3.6 .6 \mathrm{a})}{=}} f_{1}(x)+f_{2}(x) \text {. }
\end{aligned}
$$

\subsection{Lineare inhomogene Differentialgleichung zweiter Ordnung mit konstanten Koeffizien- ten}

\section{Definition 3.7.1}

Gegeben seien eine Funktion $f: I \rightarrow \mathbb{R}$, die auf einem Intervall I definiert und stetig ist, und reelle Zahlen $p, q \in \mathbb{R}$.

Gesucht ist eine Funktion $y: I \rightarrow \mathbb{R}$, sodass für alle $x \in I$ gilt

$$
y^{\prime \prime}(x)+p \cdot y^{\prime}(x)+q \cdot y(x)=f(x) .
$$

Gleichung (3.7.1) heißt lineare inhomogene Differentialgleichung zweiter Ordnung mit konstanten Koeffizienten. Die Funktion y $I \rightarrow \mathbb{R}$ heißt Lösung der Gleichung (3.7.1) auf dem Intervall I. (Vgl. Definition 3.1.1, Bemerkung 3.1.3, Definition 3.3.1). 


\section{Bemerkung 3.7.2}

Nach 3.6.2 hat die allgemeine Lösung der linearen inhomogenen Differentialgleichung (3.7.1) die Form

$$
y(x)=\widetilde{y}(x)+y^{*}(x),
$$

wobei $\widetilde{y}(x)$ die allgemeine Lösung der entsprechenden linearen homogenen Differentialgleichung

$$
y^{\prime \prime}(x)+p \cdot y^{\prime}(x)+q \cdot y(x)=0
$$

und $y^{*}(x)$ eine partikuläre Lösung der linearen inhomogenen Differentialgleichung (3.7.1) ist. Die allgemeine Lösung $\widetilde{y}(x)$ von $(3.7 .1 \mathrm{~h})$ bekommt man, wenn man die charakteristische Gleichung

$$
\lambda^{2}+p \cdot \lambda+q=0
$$

untersucht (s. Folgerungen 3.3.3, 3.3.5, 3.3.7).

Wir betrachten nun die Form partikulärer Lösungen $y^{*}(x)$ von (3.7.1) für einige Sonderfälle der rechten Seite $f: \mathbb{R} \rightarrow \mathbb{R}$, (d.h., hier gilt $I:=\mathbb{R})$ :

i) $f(x):=P_{n}(x)$, ein Polynom vom Grad $n \in \mathbb{N}_{0}$.

a) $\lambda=0$ ist keine Lösung der charakteristischen Gleichung (3.7.1c).

Wir suchen eine partikuläre Lösung in der Form

$$
y^{*}(x)=Q_{n}(x)
$$

wobei $Q_{n}(x)$ ein Polynom vom Grad n mit unbekannten Koeffizienten ist.

(s. Beispiel 3.7.3: $\left.\quad y^{\prime \prime}(x)-y^{\prime}(x)+y(x)=x^{3}+6\right)$

b) $\lambda=0$ ist eine Lösung der charakteristischen Gleichung (3.7.1c) (mit Vielfachheit $r \in \mathbb{N}$ ).

Wir suchen eine partikuläre Lösung in der Form

$$
y^{*}(x)=x^{r} \cdot Q_{n}(x)
$$

wobei $Q_{n}(x)$ ein Polynom vom Grad n mit unbekannten Koeffizienten ist.

(s. Beispiel 3.7.4: $y^{\prime \prime}(x)-2 y^{\prime}(x)=x^{2}-1$ ) 
ii) $f(x):=\mathrm{e}^{\gamma \cdot x} \cdot P_{n}(x)$, wobei $\gamma \in \mathbb{R} \backslash\{0\}$ und $P_{n}(x)$ ein Polynom vom Grad $n \in \mathbb{N}_{0}$ ist.

a) $\lambda=\gamma$ ist keine Lösung der charakteristischen Gleichung (3.7.1c).

Wir suchen eine partikuläre Lösung in der Form

$$
y^{*}(x)=\mathrm{e}^{\gamma \cdot x} \cdot Q_{n}(x)
$$

wobei $Q_{n}(x)$ ein Polynom vom Grad n mit unbekannten Koeffizienten ist. (s. Beispiel 3.7.5: $\left.\quad y^{\prime \prime}(x)+2 y^{\prime}(x)+y(x)=3 x \cdot \mathrm{e}^{2 x}\right)$

b) $\lambda=\gamma$ ist eine Lösung der charakteristischen Gleichung (3.7.1c) (mit Vielfachheit $r \in \mathbb{N}$ ).

Wir suchen eine partikuläre Lösung in der Form

$$
y^{*}(x)=x^{r} \cdot \mathrm{e}^{\gamma \cdot x} \cdot Q_{n}(x),
$$

wobei $Q_{n}(x)$ ein Polynom vom Grad n mit unbekannten Koeffizienten ist.

(s. Beispiel 3.7.6: $\left.\quad y^{\prime \prime}(x)-2 y^{\prime}(x)+y(x)=2 x \cdot \mathrm{e}^{x}\right)$

iii) $f(x):=P_{n}(x) \cdot \cos (\delta \cdot x)+Q_{m}(x) \cdot \sin (\delta \cdot x)$, wobei $\delta \in \mathbb{R} \backslash\{0\}$. Dabei sind $P_{n}(x), Q_{m}(x)$ Polynome vom Grad $n, m \in \mathbb{N}_{0}$ oder genau ein Polynom ist das Nullpolynom.

a) $\lambda= \pm \mathrm{i} \cdot \delta$ sind keine Lösungen der charakteristischen Gleichung (3.7.1c). Wir suchen eine partikuläre Lösung in der Form

$$
y^{*}(x)=S_{N}(x) \cdot \cos (\delta \cdot x)+T_{N}(x) \cdot \sin (\delta \cdot x),
$$

wobei $S_{N}(x), T_{N}(x)$ Polynome vom Grad nicht mehrals $N:=\max \{n, m\}$ mit unbekannten Koeffizienten sind.

(s. Beispiel 3.7.7: $\left.\quad y^{\prime \prime}(x)+4 y(x)=9 x \cdot \sin (x)\right)$

b) $\lambda= \pm \mathrm{i} \cdot \delta$ sind Lösungen der charakteristischen Gleichung (3.7.1c) (jeweils mit Vielfachheit $r \in \mathbb{N}$ ). Wir suchen eine partikuläre Lösung in der Form

$$
y^{*}(x)=x^{r} \cdot\left(S_{N}(x) \cdot \cos (\delta \cdot x)+T_{N}(x) \cdot \sin (\delta \cdot x)\right),
$$

wobei $S_{N}(x), T_{N}(x)$ Polynome vom Grad nicht mehrals $N:=\max \{n, m\}$ mit unbekannten Koeffizienten sind.

(s. Beispiel 3.7.8: $\quad y^{\prime \prime}(x)+y(x)=4 \cdot \cos (x)$ ) 
iv) $f(x):=\mathrm{e}^{\gamma \cdot x} \cdot\left(P_{n}(x) \cdot \cos (\delta \cdot x)+Q_{m}(x) \cdot \sin (\delta \cdot x)\right)$, wobei $\gamma, \delta \in \mathbb{R} \backslash\{0\}$. Dabei sind $P_{n}(x), Q_{m}(x)$ Polynome vom Grad $n, m \in \mathbb{N}_{0}$ oder genau ein Polynom ist das Nullpolynom.

a) $\lambda=\gamma \pm \mathrm{i} \cdot \delta$ sind keine Lösungen der charakteristischen Gleichung (3.7.1c). Wir suchen eine partikuläre Lösung in der Form

$$
y^{*}(x)=\mathrm{e}^{\gamma \cdot x} \cdot\left(S_{N}(x) \cdot \cos (\delta \cdot x)+T_{N}(x) \cdot \sin (\delta \cdot x)\right),
$$

wobei $S_{N}(x), T_{N}(x)$ Polynome vom Grad nicht mehrals $N:=\max \{n, m\}$ mit unbekannten Koeffizienten sind.

(s. Beispiel 3.7.9: $\left.\quad y^{\prime \prime}(x)+2 y^{\prime}(x)+2 y(x)=\mathrm{e}^{x} \cdot \sin (x)\right)$

b) $\lambda=\gamma \pm \mathrm{i} \cdot \delta \underline{\text { sind Lösungen }}$ der charakteristischen Gleichung (3.7.1c) (jeweils mit Vielfachheit $r \in \mathbb{N}$ ).

Wir suchen eine partikuläre Lösung in der Form

$$
y^{*}(x)=x^{r} \cdot \mathrm{e}^{\gamma \cdot x} \cdot\left(S_{N}(x) \cdot \cos (\delta \cdot x)+T_{N}(x) \cdot \sin (\delta \cdot x)\right),
$$

wobei $S_{N}(x), T_{N}(x)$ Polynome vom Grad nicht mehrals $N:=\max \{n, m\}$ mit unbekannten Koeffizienten sind.

(s. Beispiel 3.7.10: $\left.\quad y^{\prime \prime}(x)-2 y^{\prime}(x)+5 y(x)=\mathrm{e}^{x} \cdot \cos (2 x)\right)$

\section{Beispiel 3.7.3}

$$
y^{\prime \prime}(x)-y^{\prime}(x)+y(x)=x^{3}+6, x \in \mathbb{R} .
$$

1) $y^{\prime \prime}(x)-y^{\prime}(x)+y(x)=0$ ist die entsprechende homogene Gleichung. Die charakteristische Gleichung lautet

$$
\lambda^{2}-\lambda+1=0 \quad \Leftrightarrow \quad \lambda=\frac{1}{2} \pm \mathrm{i} \cdot \frac{\sqrt{3}}{2} \quad \text { (jeweils mit Vielfachheit } r=1 \text { ). }
$$

Hier gilt $\alpha=\frac{1}{2}, \beta=\frac{\sqrt{3}}{2}$ und nach Folgerung 3.3.7 ist

$$
\widetilde{y}(x)=\mathrm{e}^{\frac{x}{2}} \cdot\left(c_{1} \cdot \cos \left(\frac{\sqrt{3}}{2} x\right)+c_{2} \cdot \sin \left(\frac{\sqrt{3}}{2} x\right)\right)
$$

mit beliebigen $c_{1}, c_{2} \in \mathbb{R}$ die allgemeine Lösung der entsprechenden homogenen Gleichung auf $\mathbb{R}$. 
2) Wir haben $P_{3}(x)=x^{3}+6$. Da 0 keine Lösung unserer charakteristischen Gleichung ist, suchen wir nach Bemerkung 3.7.2 eine partikuläre Lösung der inhomogenen Gleichung in der Form

$$
y^{*}(x)=A x^{3}+B x^{2}+C x+D \quad \text { mit } A, B, C, D \in \mathbb{R} .
$$

Dann haben wir

$$
\left(y^{*}\right)^{\prime}(x)=3 A x^{2}+2 B x+C,
$$

sowie

$$
\left(y^{*}\right)^{\prime \prime}(x)=6 A x+2 B
$$

Einsetzen in die inhomogene Gleichung (3.7.3) ergibt

$$
\begin{aligned}
(6 A x+2 B)-\left(3 A x^{2}\right. & +2 B x+C) \\
+ & \left(A x^{3}+B x^{2}+C x+D\right)=x^{3}+6 \\
\Leftrightarrow \quad A x^{3}+(-3 A+B) \cdot x^{2}+(6 A- & 2 B+C) \cdot x \\
& +(2 B-C+D)=x^{3}+6
\end{aligned}
$$

Ein Koeffizientenvergleich liefert:

$$
\left\{\begin{array} { l } 
{ A = 1 } \\
{ - 3 A + B = 0 } \\
{ 6 A - 2 B + C = 0 } \\
{ 2 B - C + D = 6 }
\end{array} \Leftrightarrow \left\{\begin{array}{l}
A=1 \\
B=3 \\
C=0 \\
D=0
\end{array}\right.\right.
$$

Dann ist

$$
y^{*}(x)=x^{3}+3 x^{2}
$$

eine partikuläre Lösung und

$$
\begin{aligned}
y(x) & =\widetilde{y}(x)+y^{*}(x) \\
& =\mathrm{e}^{\frac{x}{2}} \cdot\left(c_{1} \cdot \cos \left(\frac{\sqrt{3}}{2} x\right)+c_{2} \cdot \sin \left(\frac{\sqrt{3}}{2} x\right)\right)+\left(x^{3}+3 x^{2}\right)
\end{aligned}
$$

die allgemeine Lösung der inhomogenen Gleichung (3.7.3) auf $\mathbb{R}$. 


\section{Beispiel 3.7.4}

$$
y^{\prime \prime}(x)-2 y^{\prime}(x)=x^{2}-1, x \in \mathbb{R} .
$$

1) $y^{\prime \prime}(x)-2 y^{\prime}(x)=0$ ist die entsprechende homogene Gleichung. Die charakteristische Gleichung lautet

$\lambda^{2}-2 \lambda=0 \quad \Leftrightarrow \quad \lambda=2 \quad$ oder $\quad \lambda=0 \quad$ (jeweils mit Vielfachheit $r=1$ ).

Seien $\lambda_{1}:=2, \lambda_{2}:=0$. Nach Folgerung 3.3.3 ist

$$
\widetilde{y}(x)=c_{1} \cdot \mathrm{e}^{2 x}+c_{2}
$$

mit beliebigen $c_{1}, c_{2} \in \mathbb{R}$ die allgemeine Lösung der entsprechenden homogenen Gleichung auf $\mathbb{R}$.

2) Wir haben $P_{2}(x)=x^{2}-1$. Da $\lambda=0$ eine Lösung mit Vielfachheit $r=1$ unserer charakteristischen Gleichung ist, suchen wir nach Bemerkung 3.7.2 eine partikuläre Lösung der inhomogenen Gleichung in der Form

$$
y^{*}(x)=x^{1} \cdot\left(A x^{2}+B x+C\right)=A x^{3}+B x^{2}+C x \quad \text { mit } A, B, C \in \mathbb{R} .
$$

Dann haben wir

$$
\left(y^{*}\right)^{\prime}(x)=3 A x^{2}+2 B x+C,
$$

sowie

$$
\left(y^{*}\right)^{\prime \prime}(x)=6 A x+2 B
$$

Einsetzen in die inhomogene Gleichung (3.7.4) ergibt

$$
\begin{aligned}
(6 A x+2 B)-2 \cdot\left(3 A x^{2}+2 B x+C\right) & =x^{2}-1 \\
\Leftrightarrow \quad-6 A \cdot x^{2}+(6 A-4 B) \cdot x+(2 B-2 C) & =x^{2}-1 .
\end{aligned}
$$

Ein Koeffizientenvergleich liefert:

$$
\left\{\begin{array} { l } 
{ - 6 A = 1 } \\
{ 6 A - 4 B = 0 } \\
{ 2 B - 2 C = - 1 }
\end{array} \quad \Leftrightarrow \quad \left\{\begin{array}{l}
A=-\frac{1}{6} \\
B=-\frac{1}{4} \\
C=\frac{1}{4}
\end{array}\right.\right.
$$


Dann ist

$$
y^{*}(x)=-\frac{x^{3}}{6}-\frac{x^{2}}{4}+\frac{x}{4}
$$

eine partikuläre Lösung und

$$
y(x)=\widetilde{y}(x)+y^{*}(x)=c_{1} \cdot \mathrm{e}^{2 x}+c_{2}+\left(-\frac{x^{3}}{6}-\frac{x^{2}}{4}+\frac{x}{4}\right)
$$

die allgemeine Lösung der inhomogenen Gleichung (3.7.4) auf $\mathbb{R}$.

\section{Beispiel 3.7.5}

$$
y^{\prime \prime}(x)+2 y^{\prime}(x)+y(x)=3 x \cdot \mathrm{e}^{2 x}, \quad x \in \mathbb{R} .
$$

1) $y^{\prime \prime}(x)+2 y^{\prime}(x)+y(x)=0$ ist die entsprechende homogene Gleichung. Die charakteristische Gleichung lautet

$$
\lambda^{2}+2 \lambda+1=0 \quad \Leftrightarrow \quad \lambda=-1 \quad \text { (mit Vielfachheit } \quad r=2 \text { ). }
$$

Nach Folgerung 3.3.5 ist

$$
\widetilde{y}(x)=\mathrm{e}^{-x} \cdot\left(c_{1}+c_{2} \cdot x\right)
$$

mit beliebigen $c_{1}, c_{2} \in \mathbb{R}$ die allgemeine Lösung der entsprechenden homogenen Gleichung auf $\mathbb{R}$.

2) Wir haben $\gamma=2$ und $P_{1}(x)=3 x$. Da 2 keine Lösung unserer charakteristischen Gleichung ist, suchen wir nach Bemerkung 3.7.2 eine partikuläre Lösung der inhomogenen Gleichung in der Form

$$
y^{*}(x)=(A x+B) \cdot \mathrm{e}^{2 x} \quad \text { mit } A, B \in \mathbb{R} .
$$

Dann haben wir

$$
\begin{aligned}
\left(y^{*}\right)^{\prime}(x) & =A \cdot \mathrm{e}^{2 x}+(A x+B) \cdot 2 \mathrm{e}^{2 x} \\
& =(2 A x+A+2 B) \cdot \mathrm{e}^{2 x},
\end{aligned}
$$

sowie

$$
\begin{aligned}
\left(y^{*}\right)^{\prime \prime}(x) & =2 A \cdot \mathrm{e}^{2 x}+(2 A x+A+2 B) \cdot 2 \mathrm{e}^{2 x} \\
& =(4 A x+4 A+4 B) \cdot \mathrm{e}^{2 x} .
\end{aligned}
$$


Einsetzen in die inhomogene Gleichung (3.7.5) ergibt:

$$
\begin{aligned}
& (4 A x+4 A+4 B) \cdot \mathrm{e}^{2 x}+2 \cdot(2 A x+A+2 B) \cdot \mathrm{e}^{2 x}+(A x+B) \cdot \mathrm{e}^{2 x}=3 x \cdot \mathrm{e}^{2 x}
\end{aligned}
$$

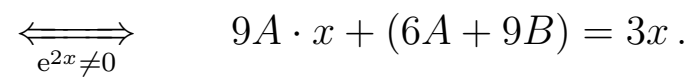

Ein Koeffizientenvergleich liefert:

$$
\left\{\begin{array} { l } 
{ 9 A = 3 } \\
{ 6 A + 9 B = 0 }
\end{array} \Leftrightarrow \left\{\begin{array}{l}
A=\frac{1}{3} \\
B=-\frac{2}{9}
\end{array}\right.\right.
$$

Dann ist

$$
y^{*}(x)=\left(\frac{x}{3}-\frac{2}{9}\right) \cdot \mathrm{e}^{2 x}
$$

eine partikuläre Lösung und

$$
y(x)=\widetilde{y}(x)+y^{*}(x)=\mathrm{e}^{-x} \cdot\left(c_{1}+c_{2} \cdot x\right)+\left(\frac{x}{3}-\frac{2}{9}\right) \cdot \mathrm{e}^{2 x}
$$

die allgemeine Lösung der inhomogenen Gleichung (3.7.5) auf $\mathbb{R}$.

\section{Beispiel 3.7.6}

$$
y^{\prime \prime}(x)-2 y^{\prime}(x)+y(x)=2 x \cdot \mathrm{e}^{x}, x \in \mathbb{R} .
$$

1) $y^{\prime \prime}(x)-2 y^{\prime}(x)+y(x)=0$ ist die entsprechende homogene Gleichung. Die charakteristische Gleichung lautet

$$
\lambda^{2}-2 \lambda+1=0 \quad \Leftrightarrow \quad \lambda=1 \quad(\text { mit Vielfachheit } r=2) .
$$

Nach Folgerung 3.3.5 ist

$$
\widetilde{y}(x)=\mathrm{e}^{x} \cdot\left(c_{1}+c_{2} \cdot x\right)
$$

mit beliebigen $c_{1}, c_{2} \in \mathbb{R}$ die allgemeine Lösung der entsprechenden homogenen Gleichung auf $\mathbb{R}$. 
2) Wir haben $\gamma=1$ und $P_{1}(x)=2 x$. Da $\lambda=1$ eine Lösung mit Vielfachheit $r=2$ unserer charakteristischen Gleichung ist, suchen wir nach Bemerkung 3.7.2 eine partikuläre Lösung der inhomogenen Gleichung in der Form

$$
y^{*}(x)=x^{2} \cdot(A x+B) \cdot \mathrm{e}^{x}=\left(A x^{3}+B x^{2}\right) \cdot \mathrm{e}^{x} \quad \text { mit } A, B \in \mathbb{R} .
$$

Dann haben wir

$$
\begin{aligned}
\left(y^{*}\right)^{\prime}(x) & =\left(3 A x^{2}+2 B x\right) \cdot \mathrm{e}^{x}+\left(A x^{3}+B x^{2}\right) \cdot \mathrm{e}^{x} \\
& =\left(A x^{3}+(3 A+B) \cdot x^{2}+2 B x\right) \cdot \mathrm{e}^{x}
\end{aligned}
$$

sowie

$$
\begin{aligned}
\left(y^{*}\right)^{\prime \prime}(x)= & \left(3 A x^{2}+(6 A+2 B) \cdot x+2 B\right) \cdot \mathrm{e}^{x} \\
& +\left(A x^{3}+(3 A+B) \cdot x^{2}+2 B x\right) \cdot \mathrm{e}^{x} \\
= & \left(A x^{3}+(6 A+B) \cdot x^{2}+(6 A+4 B) \cdot x+2 B\right) \cdot \mathrm{e}^{x} .
\end{aligned}
$$

Einsetzen in die inhomogene Gleichung (3.7.6) ergibt

$$
\begin{gathered}
\left(A x^{3}+(6 A+B) \cdot x^{2}+(6 A+4 B) \cdot x+2 B\right) \cdot \mathrm{e}^{x} \\
-2 \cdot\left(A x^{3}+(3 A+B) \cdot x^{2}+2 B x\right) \cdot \mathrm{e}^{x}+\left(A x^{3}+B x^{2}\right) \cdot \mathrm{e}^{x}=2 x \cdot \mathrm{e}^{x} \\
\qquad \underset{\mathrm{e}^{x} \neq 0}{\Longleftrightarrow} \quad A x+2 B=2 x .
\end{gathered}
$$

Ein Koeffizientenvergleich liefert:

$$
\left\{\begin{array} { l } 
{ 6 A = 2 } \\
{ 2 B = 0 }
\end{array} \Leftrightarrow \left\{\begin{array}{l}
A=\frac{1}{3} \\
B=0
\end{array}\right.\right.
$$

Dann ist

$$
y^{*}(x)=\frac{x^{3} \cdot \mathrm{e}^{x}}{3}
$$

eine partikuläre Lösung und

$$
y(x)=\widetilde{y}(x)+y^{*}(x)=\mathrm{e}^{x} \cdot\left(c_{1}+c_{2} \cdot x\right)+\frac{x^{3} \cdot \mathrm{e}^{x}}{3}
$$

die allgemeine Lösung der inhomogenen Gleichung (3.7.6) auf $\mathbb{R}$. 


\section{Beispiel 3.7.7}

$$
y^{\prime \prime}(x)+4 y(x)=9 x \cdot \sin (x), x \in \mathbb{R} .
$$

1) $y^{\prime \prime}(x)+4 y(x)=0$ ist die entsprechende homogene Gleichung. Die charakteristische Gleichung lautet

$$
\lambda^{2}+4=0 \quad \Leftrightarrow \quad \lambda= \pm \mathrm{i} \cdot 2 \quad \text { (jeweils mit Vielfachheit } r=1 \text { ). }
$$

Hier gilt $\alpha=0, \beta=2$ und nach Folgerung 3.3.7 ist

$$
\widetilde{y}(x)=c_{1} \cdot \cos (2 x)+c_{2} \cdot \sin (2 x)
$$

mit beliebigen $c_{1}, c_{2} \in \mathbb{R}$ die allgemeine Lösung der entsprechenden homogenen Gleichung auf $\mathbb{R}$.

2) Wir haben $\delta=1, P(x) \equiv 0$ (Nullpolynom) und $Q_{1}(x)=9 x$. Da $\pm \mathrm{i}$ keine Lösungen unserer charakteristischen Gleichung sind, suchen wir nach Bemerkung 3.7.2 eine partikuläre Lösung der inhomogenen Gleichung in der Form

$$
y^{*}(x)=(A x+B) \cdot \cos (x)+(C x+D) \cdot \sin (x) \quad \text { mit } A, B, C, D \in \mathbb{R} .
$$

Dann haben wir

$$
\begin{aligned}
\left(y^{*}\right)^{\prime}(x)= & A \cdot \cos (x)-(A x+B) \cdot \sin (x) \\
& +C \cdot \sin (x)+(C x+D) \cdot \cos (x) \\
= & (C x+(A+D)) \cdot \cos (x)+(-A x+(-B+C)) \cdot \sin (x),
\end{aligned}
$$

sowie

$$
\begin{aligned}
\left(y^{*}\right)^{\prime \prime}(x)= & C \cdot \cos (x)+(C x+(A+D)) \cdot(-\sin (x)) \\
& +(-A) \cdot \sin (x)+(-A x+(-B+C)) \cdot \cos (x) \\
= & (-A x+(-B+2 C)) \cdot \cos (x)+(-C x+(-2 A-D)) \cdot \sin (x) .
\end{aligned}
$$

Einsetzen in die inhomogene Gleichung (3.7.7) ergibt

$$
\begin{array}{r}
(-A x+(-B+2 C)) \cdot \cos (x)+(-C x+(-2 A-D)) \cdot \sin (x) \\
+4 \cdot((A x+B) \cdot \cos (x)+(C x+D) \cdot \sin (x))=9 x \cdot \sin (x) \\
\Leftrightarrow \quad \begin{array}{r}
3 A x \cdot \cos (x)+(3 B+2 C) \cdot \cos (x) \\
+3 C x \cdot \sin (x)+(-2 A+3 D) \cdot \sin (x)=9 x \cdot \sin (x) .
\end{array}
\end{array}
$$


Ein Koeffizientenvergleich liefert:

$$
\left\{\begin{array} { l } 
{ 3 A = 0 } \\
{ 3 B + 2 C = 0 } \\
{ 3 C = 9 } \\
{ - 2 A + 3 D = 0 }
\end{array} \quad \Leftrightarrow \quad \left\{\begin{array}{l}
A=0 \\
B=-2 \\
C=3 \\
D=0
\end{array}\right.\right.
$$

Dann ist

$$
y^{*}(x)=-2 \cdot \cos (x)+3 x \cdot \sin (x)
$$

eine partikuläre Lösung und

$$
\begin{aligned}
y(x) & =\widetilde{y}(x)+y^{*}(x) \\
& =c_{1} \cdot \cos (2 x)+c_{2} \cdot \sin (2 x)+(-2 \cdot \cos (x)+3 x \cdot \sin (x))
\end{aligned}
$$

die allgemeine Lösung der inhomogenen Gleichung (3.7.7) auf $\mathbb{R}$.

\section{Beispiel 3.7.8}

$$
y^{\prime \prime}(x)+y(x)=4 \cdot \cos (x), \quad x \in \mathbb{R} .
$$

1) $y^{\prime \prime}(x)+y(x)=0$ ist die entsprechende homogene Gleichung. Die charakteristische Gleichung lautet

$$
\lambda^{2}+1=0 \quad \Leftrightarrow \quad \lambda= \pm \mathrm{i} \quad(\text { jeweils mit Vielfachheit } \quad r=1 \text { ). }
$$

Hier gilt $\alpha=0, \beta=1$ und nach Folgerung 3.3.7 ist

$$
\widetilde{y}(x)=c_{1} \cdot \cos (x)+c_{2} \cdot \sin (x)
$$

mit beliebigen $c_{1}, c_{2} \in \mathbb{R}$ die allgemeine Lösung der entsprechenden homogenen Gleichung auf $\mathbb{R}$.

2) Wir haben $\delta=1, P_{0}(x)=4$ und $Q(x) \equiv 0$ (Nullpolynom). Da $\lambda= \pm \mathrm{i}$ Lösungen (jeweils mit Vielfachheit $r=1$ ) unserer charakteristischen Gleichung sind, suchen wir nach Bemerkung 3.7.2 eine partikuläre Lösung der inhomogenen Gleichung in der Form

$$
y^{*}(x)=x \cdot(A \cdot \cos (x)+B \cdot \sin (x)) \quad \text { mit } A, B \in \mathbb{R} .
$$


Dann haben wir

$$
\begin{aligned}
\left(y^{*}\right)^{\prime}(x) & =A \cdot \cos (x)+B \cdot \sin (x)+x \cdot(-A \cdot \sin (x)+B \cdot \cos (x)) \\
& =(A+B x) \cdot \cos (x)+(B-A x) \cdot \sin (x)
\end{aligned}
$$

sowie

$$
\begin{aligned}
\left(y^{*}\right)^{\prime \prime}(x)= & B \cdot \cos (x)+(A+B x) \cdot(-\sin (x)) \\
& +(-A) \cdot \sin (x)+(B-A x) \cdot \cos (x) \\
= & (-A x+2 B) \cdot \cos (x)+(-B x-2 A) \cdot \sin (x) .
\end{aligned}
$$

Einsetzen in die inhomogene Gleichung (3.7.8) ergibt

$$
\begin{aligned}
(-A x+2 B) \cdot \cos (x)+( & (B x-2 A) \cdot \sin (x) \\
+x \cdot(A \cdot \cos (x)+B \cdot \sin (x)) & =4 \cdot \cos (x) \\
\Leftrightarrow \quad & 2 B \cdot \cos (x)-2 A \cdot \sin (x)=4 \cdot \cos (x) .
\end{aligned}
$$

Ein Koeffizientenvergleich liefert:

$$
\left\{\begin{array} { l } 
{ 2 B = 4 } \\
{ - 2 A = 0 }
\end{array} \Leftrightarrow \left\{\begin{array}{l}
A=0 \\
B=2
\end{array}\right.\right.
$$

Dann ist $y^{*}(x)=2 x \cdot \sin (x)$ eine partikuläre Lösung und

$$
y(x)=\widetilde{y}(x)+y^{*}(x)=c_{1} \cdot \cos (x)+c_{2} \cdot \sin (x)+2 x \cdot \sin (x)
$$

die allgemeine Lösung der inhomogenen Gleichung (3.7.8) auf $\mathbb{R}$.

\section{Beispiel 3.7.9}

$$
y^{\prime \prime}(x)+2 y^{\prime}(x)+2 y(x)=\mathrm{e}^{x} \cdot \sin (x), x \in \mathbb{R} .
$$

1) $y^{\prime \prime}(x)+2 y^{\prime}(x)+2 y(x)=0$ ist die entsprechende homogene Gleichung. Die charakteristische Gleichung lautet

$$
\left.\lambda^{2}+2 \lambda+2=0 \quad \Leftrightarrow \quad \lambda=-1 \pm \mathrm{i} \quad \text { (jeweils mit Vielfachheit } r=1\right) .
$$

Hier gilt $\alpha=-1, \beta=1$ und nach Folgerung 3.3.7 ist

$$
\widetilde{y}(x)=\mathrm{e}^{-x} \cdot\left(c_{1} \cdot \cos (x)+c_{2} \cdot \sin (x)\right)
$$

mit beliebigen $c_{1}, c_{2} \in \mathbb{R}$ die allgemeine Lösung der entsprechenden homogenen Gleichung auf $\mathbb{R}$. 
2) Wir haben $\gamma=1, \delta=1, P(x) \equiv 0$ (Nullpolynom) und $Q_{0}(x)=1$. Da $1 \pm \mathrm{i}$ keine Lösungen unserer charakteristischen Gleichung sind, suchen wir nach Bemerkung 3.7.2 eine partikuläre Lösung der inhomogenen Gleichung in der Form

$$
y^{*}(x)=\mathrm{e}^{x} \cdot(A \cdot \cos (x)+B \cdot \sin (x)) \quad \text { mit } A, B \in \mathbb{R} .
$$

Dann haben wir

$$
\begin{aligned}
\left(y^{*}\right)^{\prime}(x)= & \mathrm{e}^{x} \cdot(A \cdot \cos (x)+B \cdot \sin (x)) \\
& \quad+\mathrm{e}^{x} \cdot(-A \cdot \sin (x)+B \cdot \cos (x)) \\
= & \mathrm{e}^{x} \cdot((A+B) \cdot \cos (x)+(-A+B) \cdot \sin (x)),
\end{aligned}
$$

sowie

$$
\begin{aligned}
\left(y^{*}\right)^{\prime \prime}(x)= & \mathrm{e}^{x} \cdot((A+B) \cdot \cos (x)+(-A+B) \cdot \sin (x)) \\
& \quad+\mathrm{e}^{x} \cdot(-(A+B) \cdot \sin (x)+(-A+B) \cdot \cos (x)) \\
= & \mathrm{e}^{x} \cdot(2 B \cdot \cos (x)+(-2 A) \cdot \sin (x))
\end{aligned}
$$

Einsetzen in die inhomogene Gleichung (3.7.9) ergibt

$$
\begin{aligned}
& \mathrm{e}^{x} \cdot(2 B \cdot \cos (x)-2 A \cdot \sin (x)) \\
& +2 \mathrm{e}^{x} \cdot((A+B) \cdot \cos (x)+(-A+B) \cdot \sin (x)) \\
& \quad+2 \mathrm{e}^{x} \cdot(A \cdot \cos (x)+B \cdot \sin (x))=\mathrm{e}^{x} \cdot \sin (x) \\
& \underset{\mathrm{e}^{x} \neq 0}{\longrightarrow} \quad(4 A+4 B) \cdot \cos (x)+(-4 A+4 B) \cdot \sin (x)=\sin (x) .
\end{aligned}
$$

Ein Koeffizientenvergleich liefert:

$$
\left\{\begin{array} { l } 
{ 4 A + 4 B = 0 } \\
{ - 4 A + 4 B = 1 }
\end{array} \Leftrightarrow \left\{\begin{array}{l}
A=-\frac{1}{8} \\
B=\frac{1}{8}
\end{array}\right.\right.
$$

Dann ist

$$
y^{*}(x)=\mathrm{e}^{x} \cdot\left(-\frac{1}{8} \cdot \cos (x)+\frac{1}{8} \cdot \sin (x)\right)
$$

eine partikuläre Lösung und

$$
\begin{aligned}
y(x) & =\widetilde{y}(x)+y^{*}(x) \\
& =\mathrm{e}^{-x} \cdot\left(c_{1} \cdot \cos (x)+c_{2} \cdot \sin (x)\right)+\mathrm{e}^{x} \cdot\left(-\frac{1}{8} \cdot \cos (x)+\frac{1}{8} \cdot \sin (x)\right)
\end{aligned}
$$

die allgemeine Lösung der inhomogenen Gleichung (3.7.9) auf $\mathbb{R}$. 


\section{Beispiel 3.7.10}

$$
y^{\prime \prime}(x)-2 y^{\prime}(x)+5 y(x)=\mathrm{e}^{x} \cdot \cos (2 x), \quad x \in \mathbb{R} .
$$

1) $y^{\prime \prime}(x)-2 y^{\prime}(x)+5 y(x)=0$ ist die entsprechende homogene Gleichung. Die charakteristische Gleichung lautet

$$
\lambda^{2}-2 \lambda+5=0 \quad \Leftrightarrow \quad \lambda=1 \pm \mathrm{i} \cdot 2 \quad \text { (jeweils mit Vielfachheit } r=1 \text { ). }
$$

Hier gilt $\alpha=1, \beta=2$ und nach Folgerung 3.3.7 ist

$$
\widetilde{y}(x)=\mathrm{e}^{x} \cdot\left(c_{1} \cdot \cos (2 x)+c_{2} \cdot \sin (2 x)\right)
$$

mit beliebigen $c_{1}, c_{2} \in \mathbb{R}$ die allgemeine Lösung der entsprechenden homogenen Gleichung auf $\mathbb{R}$.

2) Wir haben $\gamma=1, \delta=2, P_{0}(x)=1$ und $Q(x) \equiv 0$ (Nullpolynom). Da $\lambda=1 \pm \mathrm{i} \cdot 2 \quad$ Lösungen (jeweils mit Vielfachheit $r=1$ ) unserer charakteristischen Gleichung sind, suchen wir nach Bemerkung 3.7.2 eine partikuläre Lösung der inhomogenen Gleichung in der Form

$$
\begin{aligned}
y^{*}(x) & =x \cdot \mathrm{e}^{x} \cdot(A \cdot \cos (2 x)+B \cdot \sin (2 x)) \\
& =\mathrm{e}^{x} \cdot(A x \cdot \cos (2 x)+B x \cdot \sin (2 x)) \quad \text { mit } A, B \in \mathbb{R} .
\end{aligned}
$$

Dann haben wir

$$
\begin{aligned}
&\left(y^{*}\right)^{\prime}(x)=\mathrm{e}^{x} \cdot(A x \cdot \cos (2 x)+B x \cdot \sin (2 x)) \\
&+\mathrm{e}^{x} \cdot(A \cdot \cos (2 x)-2 A x \cdot \sin (2 x) \\
&\quad+B \cdot \sin (2 x)+2 B x \cdot \cos (2 x)) \\
&=\mathrm{e}^{x} \cdot((A x+A+2 B x) \cdot \cos (2 x) \\
&\quad+(B x-2 A x+B) \cdot \sin (2 x)) \\
&=\mathrm{e}^{x} \cdot(((A+2 B) x+A) \cdot \cos (2 x) \\
&\quad+((B-2 A) x+B) \cdot \sin (2 x))
\end{aligned}
$$


sowie

$$
\begin{aligned}
\left(y^{*}\right)^{\prime \prime}(x)=\mathrm{e}^{x} \cdot(((A+2 B) x+A) \cdot \cos (2 x) & +((B-2 A) x+B) \cdot \sin (2 x)) \\
+\mathrm{e}^{x} \cdot((A+2 B) \cdot \cos (2 x) & +((A+2 B) x+A) \cdot(-\sin (2 x)) \cdot 2 \\
& +(B-2 A) \cdot \sin (2 x) \\
& +((B-2 A) \cdot x+B) \cdot \cos (2 x) \cdot 2) \\
=\mathrm{e}^{x} \cdot(((-3 A+4 B) x+(2 A+4 B)) \cdot \cos (2 x) & +((-4 A-3 B) x+(-4 A+2 B)) \cdot \sin (2 x)) .
\end{aligned}
$$

Einsetzen in die inhomogene Gleichung (3.7.10) ergibt

$$
\begin{aligned}
& \mathrm{e}^{x} \cdot(((-3 A+4 B) x+(2 A+4 B)) \cdot \cos (2 x) \\
& +((-4 A-3 B) x+(-4 A+2 B)) \cdot \sin (2 x)) \\
& -2 \mathrm{e}^{x} \cdot(((A+2 B) x+A) \cdot \cos (2 x) \\
& +((B-2 A) x+B) \cdot \sin (2 x)) \\
& +5 \mathrm{e}^{x} \cdot(A x \cdot \cos (2 x)+B x \cdot \sin (2 x))=\mathrm{e}^{x} \cdot \cos (2 x) \\
& \Longleftrightarrow \quad 4 B \cdot \cos (2 x)+(-4 A) \cdot \sin (2 x)=\cos (2 x) .
\end{aligned}
$$

Ein Koeffizientenvergleich liefert:

$$
\left\{\begin{array} { l } 
{ 4 B = 1 } \\
{ - 4 A = 0 }
\end{array} \Leftrightarrow \left\{\begin{array}{l}
B=\frac{1}{4} \\
A=0
\end{array}\right.\right.
$$

Dann ist

$$
y^{*}(x)=\frac{\mathrm{e}^{x} \cdot x \cdot \sin (2 x)}{4}
$$

eine partikuläre Lösung und

$$
\begin{aligned}
y(x) & =\widetilde{y}(x)+y^{*}(x) \\
& =\mathrm{e}^{x} \cdot\left(c_{1} \cdot \cos (2 x)+c_{2} \cdot \sin (2 x)\right)+\frac{\mathrm{e}^{x} \cdot x \cdot \sin (2 x)}{4}
\end{aligned}
$$

die allgemeine Lösung der inhomogenen Gleichung (3.7.10) auf $\mathbb{R}$. 


\section{Beispiel 3.7.11}

$$
y^{\prime \prime}(x)-5 y^{\prime}(x)+6 y(x)=\cos (3 x)+x \cdot \mathrm{e}^{3 x}, \quad x \in \mathbb{R} .
$$

1) $y^{\prime \prime}(x)-5 y^{\prime}(x)+6 y(x)=0$ ist die entsprechende homogene Gleichung.

Die charakteristische Gleichung lautet

$$
\lambda^{2}-5 \lambda+6=0 \Leftrightarrow \lambda=2 \text { oder } \lambda=3 \text { (jeweils mit Vielfachheit } r=1 \text { ). }
$$

Seien $\lambda_{1}:=2$ und $\lambda_{2}:=3$. Nach Folgerung 3.3.3 ist

$$
\widetilde{y}(x)=c_{1} \cdot \mathrm{e}^{2 x}+c_{2} \cdot \mathrm{e}^{3 x}
$$

mit beliebigen $c_{1}, c_{2} \in \mathbb{R}$ die allgemeine Lösung der entsprechenden homogenen Gleichung auf $\mathbb{R}$.

2) Nach Bemerkung 3.7.2 suchen wir eine partikuläre Lösung der inhomogenen Gleichung

$$
y^{\prime \prime}(x)-5 y^{\prime}(x)+6 y(x)=\cos (3 x)
$$

in der Form

$$
y_{1}^{*}(x)=A \cdot \cos (3 x)+B \cdot \sin (3 x) \quad \text { mit } A, B \in \mathbb{R} .
$$

Dann haben wir

$$
\left(y_{1}^{*}\right)^{\prime}(x)=-3 A \cdot \sin (3 x)+3 B \cdot \cos (3 x),
$$

sowie

$$
\left(y_{1}^{*}\right)^{\prime \prime}(x)=-9 A \cdot \cos (3 x)-9 B \cdot \sin (3 x) \text {. }
$$

Einsetzen in die inhomogene Gleichung (3.7.11a) ergibt

$$
\begin{aligned}
&-9 A \cdot \cos (3 x)-9 B \cdot \sin (3 x) \\
&-5 \cdot(-3 A \cdot\sin (3 x)+3 B) \cdot \cos (3 x)) \\
&+6 \cdot(A \cdot \cos (3 x)+B \cdot \sin (3 x))=\cos (3 x) \\
& \Leftrightarrow \quad(-3 A-15 B) \cdot \cos (3 x)+(15 A-3 B) \cdot \sin (3 x)=\cos (3 x) .
\end{aligned}
$$


Ein Koeffizientenvergleich liefert:

$$
\left\{\begin{array} { l } 
{ - 3 A - 1 5 B = 1 } \\
{ 1 5 A - 3 B = 0 }
\end{array} \Leftrightarrow \left\{\begin{array}{l}
A=-\frac{1}{78} \\
B=-\frac{5}{78}
\end{array}\right.\right.
$$

Dann ist

$$
y_{1}^{*}(x)=-\frac{1}{78} \cdot \cos (3 x)-\frac{5}{78} \cdot \sin (3 x)
$$

eine partikuläre Lösung der inhomogenen Gleichung (3.7.11a) auf $\mathbb{R}$.

3) Nach Bemerkung 3.7.2 suchen wir eine partikuläre Lösung der inhomogenen Gleichung

$$
y^{\prime \prime}(x)-5 y^{\prime}(x)+6 y(x)=x \cdot \mathrm{e}^{3 x}
$$

in der Form

$$
y_{2}^{*}(x)=x \cdot(A x+B) \cdot \mathrm{e}^{3 x}=\left(A x^{2}+B x\right) \cdot \mathrm{e}^{3 x} \quad \text { mit } A, B \in \mathbb{R} .
$$

Dann haben wir

$$
\begin{aligned}
\left(y_{2}^{*}\right)^{\prime}(x) & =(2 A x+B) \cdot \mathrm{e}^{3 x}+\left(A x^{2}+B x\right) \cdot \mathrm{e}^{3 x} \cdot 3 \\
& =\left(3 A x^{2}+(2 A+3 B) x+B\right) \cdot \mathrm{e}^{3 x},
\end{aligned}
$$

sowie

$$
\begin{aligned}
\left(y_{2}^{*}\right)^{\prime \prime}(x) & =(6 A x+(2 A+3 B)) \cdot \mathrm{e}^{3 x}+\left(3 A x^{2}+(2 A+3 B) x+B\right) \cdot \mathrm{e}^{3 x} \cdot 3 \\
& =\left(9 A x^{2}+(12 A+9 B) x+(2 A+6 B) \cdot \mathrm{e}^{3 x}\right.
\end{aligned}
$$

Einsetzen in die inhomogene Gleichung (3.7.11b) ergibt

$$
\begin{aligned}
\left(9 A x^{2}+(12 A+9 B) x+(2 A+6 B)\right) \cdot \mathrm{e}^{3 x} & \\
-5 \cdot\left(3 A x^{2}+(2 A+3 B) x+B\right) \cdot \mathrm{e}^{3 x} & \\
& +6 \cdot\left(A x^{2}+B x\right) \cdot \mathrm{e}^{3 x}=x \cdot \mathrm{e}^{3 x} \\
& 2 A x+(2 A+B)=x .
\end{aligned}
$$

Ein Koeffizientenvergleich liefert:

$$
\left\{\begin{array} { l } 
{ 2 A = 1 } \\
{ 2 A + B = 0 }
\end{array} \Leftrightarrow \left\{\begin{array}{l}
A=\frac{1}{2} \\
B=-1
\end{array}\right.\right.
$$


Dann ist

$$
y_{2}^{*}(x)=\left(\frac{x^{2}}{2}-x\right) \cdot \mathrm{e}^{3 x}
$$

eine partikuläre Lösung der inhomogenen Gleichung (3.7.11b) auf $\mathbb{R}$.

4) Nach dem Superpositionsprinzip (Satz 3.6.6) ist

$$
y^{*}(x)=y_{1}^{*}(x)+y_{2}^{*}(x)
$$

eine partikuläre Lösung der inhomogenen Gleichung (3.7.11) und

$$
\begin{aligned}
y(x) & =\widetilde{y}(x)+y^{*}(x) \\
& =c_{1} \cdot \mathrm{e}^{2 x}+c_{2} \cdot \mathrm{e}^{3 x}+\left(-\frac{1}{78} \cdot \cos (3 x)-\frac{5}{78} \cdot \sin (3 x)\right)+\left(\frac{x^{2}}{2}-x\right) \cdot \mathrm{e}^{3 x}
\end{aligned}
$$

die allgemeine Lösung der Gleichung (3.7.11) auf $\mathbb{R}$.

\section{Beispiel 3.7.12 (Anfangswertproblem)}

$$
\left\{\begin{aligned}
y^{\prime \prime}(x)-6 y^{\prime}(x)+8 y(x) & =\frac{4}{1+\mathrm{e}^{-2 x}}, \quad x \in \mathbb{R}, \\
y(0) & =1+2 \ln (2), \\
y^{\prime}(0) & =6 \ln (2) .
\end{aligned}\right.
$$

1) $y^{\prime \prime}(x)-6 y^{\prime}(x)+8 y(x)=0$ ist die entsprechende homogene Gleichung.

Die charakteristische Gleichung lautet

$$
\left.\lambda^{2}-6 \lambda+8=0 \Leftrightarrow \lambda=2 \text { oder } \lambda=4 \text { (jeweils mit Vielfachheit } r=1\right) \text {. }
$$

Seien $\lambda_{1}:=2$ und $\lambda_{2}:=4$. Nach Folgerung 3.3.3 ist

$$
\widetilde{y}(x)=c_{1} \cdot \mathrm{e}^{2 x}+c_{2} \cdot \mathrm{e}^{4 x}
$$

mit beliebigen $c_{1}, c_{2} \in \mathbb{R}$ die allgemeine Lösung der entsprechenden homogenen Gleichung auf $\mathbb{R}$. Die Funktionen

$$
y_{1}(x):=\mathrm{e}^{2 x}, \quad y_{2}(x):=\mathrm{e}^{4 x}
$$

bilden ein Fundamentalsystem der homogenen Gleichung auf $\mathbb{R}$. 
2) Nach dem Verfahren der Variation der Konstanten (Bemerkung 3.6.4) suchen wir eine partikuläre Lösung der inhomogenen Gleichung

$$
y^{\prime \prime}(x)-6 y^{\prime}(x)+8 y(x)=\frac{4}{1+\mathrm{e}^{-2 x}}
$$

in der Form

$$
y^{*}(x)=c_{1}(x) \cdot y_{1}(x)+c_{2}(x) \cdot y_{2}(x)
$$

Nach Bemerkung 3.6.4 folgt:

$$
\left\{\begin{array}{l}
c_{1}^{\prime}(x) \cdot y_{1}(x)+c_{2}^{\prime}(x) \cdot y_{2}(x)=0 \\
c_{1}^{\prime}(x) \cdot y_{1}^{\prime}(x)+c_{2}^{\prime}(x) \cdot y_{2}^{\prime}(x)=\frac{4}{1+\mathrm{e}^{-2 x}} .
\end{array}\right.
$$

Also gilt:

$$
\left\{\begin{array}{c}
c_{1}^{\prime}(x) \cdot \mathrm{e}^{2 x}+c_{2}^{\prime}(x) \cdot \mathrm{e}^{4 x}=0, \\
2 c_{1}^{\prime}(x) \cdot \mathrm{e}^{2 x}+4 c_{2}^{\prime}(x) \cdot \mathrm{e}^{4 x}=\frac{4}{1+\mathrm{e}^{-2 x}} .
\end{array}\right.
$$

Mit Hilfe der CRAmer'schen Regel erhalten wir:

$$
\Delta:=\left|\begin{array}{cc}
\mathrm{e}^{2 x} & \mathrm{e}^{4 x} \\
2 \mathrm{e}^{2 x} & 4 \mathrm{e}^{4 x}
\end{array}\right|=2 \cdot \mathrm{e}^{6 x} \neq 0 \quad \text { für alle } x \in \mathbb{R}
$$

und

$$
\Delta_{1}:=\left|\begin{array}{cc}
0 & \mathrm{e}^{4 x} \\
\frac{4}{1+\mathrm{e}^{-2 x}} & 4 \mathrm{e}^{4 x}
\end{array}\right|=-\frac{4 \cdot \mathrm{e}^{4 x}}{1+\mathrm{e}^{-2 x}},
$$

sowie

$$
\triangle_{2}:=\left|\begin{array}{cc}
\mathrm{e}^{2 x} & 0 \\
2 \mathrm{e}^{2 x} & \frac{4}{1+\mathrm{e}^{-2 x}}
\end{array}\right|=\frac{4 \cdot \mathrm{e}^{2 x}}{1+\mathrm{e}^{-2 x}} .
$$

Weiter gilt:

$$
c_{1}^{\prime}(x)=\frac{\triangle_{1}}{\triangle}=-\frac{2 \cdot \mathrm{e}^{-2 x}}{1+\mathrm{e}^{-2 x}}
$$


und

$$
c_{2}^{\prime}(x)=\frac{\triangle_{2}}{\triangle}=\frac{2 \cdot \mathrm{e}^{-4 x}}{1+\mathrm{e}^{-2 x}}=2 \cdot \mathrm{e}^{-2 x}-\frac{2 \cdot \mathrm{e}^{-2 x}}{1+\mathrm{e}^{-2 x}} .
$$

Dann sind

$$
c_{1}(x)=\ln \left(1+\mathrm{e}^{-2 x}\right)+m
$$

und

$$
c_{2}(x)=-\mathrm{e}^{-2 x}+\ln \left(1+\mathrm{e}^{-2 x}\right)+n
$$

mit beliebigen $m, n \in \mathbb{R}$ entsprechende Stammfunktionen.

Mit $m=0, n=0$ bekommen wir folgende partikuläre Lösung der inhomogenen Gleichung

$$
\begin{aligned}
y^{*}(x) & =c_{1}(x) \cdot y_{1}(x)+c_{2}(x) \cdot y_{2}(x) \\
& =\mathrm{e}^{2 x} \cdot \ln \left(1+\mathrm{e}^{-2 x}\right)+\left(-\mathrm{e}^{2 x}+\mathrm{e}^{4 x} \cdot \ln \left(1+\mathrm{e}^{-2 x}\right)\right) .
\end{aligned}
$$

Die allgemeine Lösung der inhomogenen Differentialgleichung auf $\mathbb{R}$ ist

$$
\begin{aligned}
y(x) & =\widetilde{y}(x)+y^{*}(x) \\
& =\widetilde{c}_{1} \cdot \mathrm{e}^{2 x}+\widetilde{c}_{2} \cdot \mathrm{e}^{4 x}+\mathrm{e}^{2 x} \cdot \ln \left(1+\mathrm{e}^{-2 x}\right)+\left(-\mathrm{e}^{2 x}+\mathrm{e}^{4 x} \cdot \ln \left(1+\mathrm{e}^{-2 x}\right)\right)
\end{aligned}
$$

mit $\widetilde{c}_{1}, \widetilde{c}_{2} \in \mathbb{R}$. Für jedes $x \in \mathbb{R}$ gilt:

$$
\begin{aligned}
y^{\prime}(x)=2 \widetilde{c}_{1} \cdot \mathrm{e}^{2 x}+4 \widetilde{c}_{2} \cdot \mathrm{e}^{4 x}+2 \cdot \mathrm{e}^{2 x} \cdot \ln \left(1+\mathrm{e}^{-2 x}\right)+\mathrm{e}^{2 x} \cdot \frac{(-2) \cdot \mathrm{e}^{-2 x}}{1+\mathrm{e}^{-2 x}} \\
\quad-2 \cdot \mathrm{e}^{2 x}+4 \cdot \mathrm{e}^{4 x} \cdot \ln \left(1+\mathrm{e}^{-2 x}\right)+\mathrm{e}^{4 x} \cdot \frac{(-2) \cdot \mathrm{e}^{-2 x}}{1+\mathrm{e}^{-2 x}} \\
=2 \widetilde{c}_{1} \cdot \mathrm{e}^{2 x}+4 \widetilde{c}_{2} \cdot \mathrm{e}^{4 x}+\mathrm{e}^{2 x} \cdot\left(2 \ln \left(1+\mathrm{e}^{-2 x}\right)+\frac{-2 \mathrm{e}^{-2 x}}{1+\mathrm{e}^{-2 x}}-2\right) \\
+\mathrm{e}^{4 x} \cdot\left(4 \ln \left(1+\mathrm{e}^{-2 x}\right)+\frac{-2 \mathrm{e}^{-2 x}}{1+\mathrm{e}^{-2 x}}\right) .
\end{aligned}
$$

Mit Hilfe der Anfangswerte können wir nun die Konstanten $\widetilde{c}_{1}, \widetilde{c}_{2} \in \mathbb{R}$ eindeutig bestimmen. Wir haben

$$
\left\{\begin{array}{l}
y(0)=1+2 \ln (2) \\
y^{\prime}(0)=6 \ln (2)
\end{array}\right.
$$


und somit gilt

$$
\begin{array}{r}
\widetilde{c}_{1}+\widetilde{c}_{2}+\ln (2)+(-1+\ln (2))=1+2 \ln (2) \\
2 \widetilde{c}_{1}+4 \widetilde{c}_{2}+(2 \ln (2)-1-2)+(4 \ln (2)-1)=6 \ln (2) \\
\Leftrightarrow\left\{\begin{array} { r } 
{ \widetilde { c } _ { 1 } + \widetilde { c } _ { 2 } = 2 } \\
{ 2 \widetilde { c } _ { 1 } + 4 \widetilde { c } _ { 2 } = 4 }
\end{array} \Leftrightarrow \left\{\begin{array}{l}
\widetilde{c}_{1}=2 \\
\widetilde{c}_{2}=0
\end{array}\right.\right.
\end{array}
$$

Dann ist

$$
\begin{aligned}
y(x) & =2 \mathrm{e}^{2 x}+\mathrm{e}^{2 x} \cdot \ln \left(1+\mathrm{e}^{-2 x}\right)-\mathrm{e}^{2 x}+\mathrm{e}^{4 x} \cdot \ln \left(1+\mathrm{e}^{-2 x}\right) \\
& =\mathrm{e}^{2 x}+\left(\mathrm{e}^{2 x}+\mathrm{e}^{4 x}\right) \cdot \ln \left(1+\mathrm{e}^{-2 x}\right)
\end{aligned}
$$

die eindeutige Lösung des Anfangswertproblems (3.7.12). 


\section{Lineare Differenzengleichungen zweiter Ordnung}

\subsection{Anfangswertproblem für lineare Differenzen- gleichung zweiter Ordnung}

Vorbemerkung: Im Folgenden werden wir Differenzengleichungen auf der Menge $\mathbb{N}_{0}$ untersuchen. Analoge Betrachtungen lassen sich auf $\mathbb{Z}^{\geqslant n_{0}}:=$ $\left\{n_{0}, n_{0}+1, \ldots\right\}$ mit einem beliebigen $n_{0} \in \mathbb{Z}$ oder auf der ganzen Menge $\mathbb{Z}$ durchführen.

\section{Definition 4.1.1}

Gegeben seien die Funktionen $p, q, f: \mathbb{N}_{0} \rightarrow \mathbb{R}$, wobei $q(n) \neq 0$ für alle $n \in \mathbb{N}_{0}$ ist. Gesucht ist eine Funktion $x: \mathbb{N}_{0} \rightarrow \mathbb{R}$, sodass für alle $n \in \mathbb{N}_{0}$ gilt:

$$
x(n+2)+p(n) \cdot x(n+1)+q(n) \cdot x(n)=f(n) .
$$

Gleichung (4.1.1) heißt lineare Differenzengleichung zweiter Ordnung. Die Funktion $x: \mathbb{N}_{0} \rightarrow \mathbb{R}$ heißt Lösung der Gleichung (4.1.1).

\section{Satz und Definition 4.1.2}

Gegeben seien die Funktionen $p, q, f: \mathbb{N}_{0} \rightarrow \mathbb{R}$, wobei $q(n) \neq 0$ für alle $n \in \mathbb{N}_{0}$ ist. Sei $n_{0} \in \mathbb{N}_{0}$ beliebig. Dann existiert für beliebige $\mu_{0}, \mu_{1} \in \mathbb{R}$ genau eine Lösung des Anfangswertproblems:

$$
\left\{\begin{aligned}
x(n+2)+p(n) \cdot x(n+1)+q(n) \cdot x(n) & =f(n), \\
x\left(n_{0}\right) & =\mu_{0}, \\
x\left(n_{0}+1\right) & =\mu_{1} .
\end{aligned}\right.
$$


BEWEIS:

\section{Existenz:}

Sind die Werte $x\left(n_{0}\right)$ und $x\left(n_{0}+1\right)$ gegeben, so ist auch der Wert an der Stelle $\left(n_{0}+2\right)$ bereits bestimmt:

$$
x\left(n_{0}+2\right)=f\left(n_{0}\right)-p\left(n_{0}\right) \cdot x\left(n_{0}+1\right)-q\left(n_{0}\right) \cdot x\left(n_{0}\right) .
$$

Für $\left(n_{0}-1\right) \in \mathbb{N}_{0}$ erhalten wir aus der Gültigkeit der Differenzengleichung

$$
x\left(n_{0}-1\right)=\frac{f\left(n_{0}-1\right)-x\left(n_{0}+1\right)-p\left(n_{0}-1\right) \cdot x\left(n_{0}\right)}{q\left(n_{0}-1\right)} .
$$

Sukzessive kann also jeder Wert von $x($.$) auf \mathbb{N}_{0}$ bestimmt werden und es existiert eine Lösung des Anfangswertproblems.

\section{Eindeutigkeit:}

Seien $x_{1}, x_{2}: \mathbb{N}_{0} \rightarrow \mathbb{R}$ zwei Lösungen des Anfangswertproblems. Dann gilt:

$$
\left\{\begin{aligned}
x_{1}(n+2)+p(n) \cdot x_{1}(n+1)+q(n) \cdot x_{1}(n) & =f(n), \\
x_{1}\left(n_{0}\right) & =\mu_{0}, \\
x_{1}\left(n_{0}+1\right) & =\mu_{1},
\end{aligned}\right.
$$

und

$$
\left\{\begin{aligned}
x_{2}(n+2)+p(n) \cdot x_{2}(n+1)+q(n) \cdot x_{2}(n) & =f(n), \\
x_{2}\left(n_{0}\right) & =\mu_{0}, \\
x_{2}\left(n_{0}+1\right) & =\mu_{1} .
\end{aligned}\right.
$$

Für $\widetilde{x}(n):=x_{1}(n)-x_{2}(n)$ mit $n \in \mathbb{N}_{0}$ folgt:

$$
\left\{\begin{aligned}
\widetilde{x}(n+2)+p(n) \cdot \widetilde{x}(n+1)+q(n) \cdot \widetilde{x}(n) & =0 \\
\widetilde{x}\left(n_{0}\right) & =0 \\
\widetilde{x}\left(n_{0}+1\right) & =0 .
\end{aligned}\right.
$$

Wir beweisen, dass (4.1.3) nur die triviale Lösung $\widetilde{x}(n)=0$ für alle $n \in \mathbb{N}_{0}$ zulässt.

Wir zeigen zuerst, dass für alle $n \in \mathbb{N}_{0}$ mit $n \geqslant n_{0}$ bereits $\widetilde{x}(n)=0$ gelten muss: 
Mit den Anfangsbedingungen haben wir schon $\widetilde{x}\left(n_{0}\right)=\widetilde{x}\left(n_{0}+1\right)=0$. Angenommen, es existiert ein $n \in \mathbb{N}$ mit $n \geqslant\left(n_{0}+2\right)$, sodass $\widetilde{x}(n) \neq 0$ gilt. Dann ist die Menge $\left\{n \in \mathbb{N}: n \geqslant\left(n_{0}+2\right)\right.$ und $\left.\widetilde{x}(n) \neq 0\right\}$ nichtleer.

Sei $s:=\min \left\{n \in \mathbb{N}: n \geqslant\left(n_{0}+2\right)\right.$ und $\left.\widetilde{x}(n) \neq 0\right\}$.

Dann gilt $\widetilde{x}(s-1)=\widetilde{x}(s-2)=0$ und nach (4.1.3) folgt:

$$
\widetilde{x}(s)=-p(s-2) \cdot \underbrace{\widetilde{x}(s-1)}_{=0}-q(s-2) \cdot \underbrace{\widetilde{x}(s-2)}_{=0}=0,
$$

im Widerspruch zur Auswahl von $s$. Also ist $\widetilde{x}(n)=0$ für alle $n \in \mathbb{N}_{0}$ mit $n \geqslant n_{0}$.

Ist $n_{0}=0$, so folgt somit sofort $\widetilde{x}(n)=0$ für alle $n \in \mathbb{N}_{0}$.

Jetzt zeigen wir, dass auch für $n_{0} \geqslant 1$ die Behauptung $\widetilde{x}(n)=0$ für alle $n \in \mathbb{N}_{0}$ mit $n \leqslant\left(n_{0}-1\right)$ folgt:

Andernfalls wäre die Menge $\left\{n \in \mathbb{N}_{0}: n \leqslant\left(n_{0}-1\right)\right.$ und $\left.\widetilde{x}(n) \neq 0\right\}$ nichtleer und es existierte $t:=\max \left\{n \in \mathbb{N}_{0}: n \leqslant\left(n_{0}-1\right)\right.$ und $\widetilde{x}(n) \neq$ $0\}$. Dann gilt $\widetilde{x}(t+1)=\widetilde{x}(t+2)=0$. Wegen

$$
\underbrace{\widetilde{x}(t+2)}_{=0}+p(t) \cdot \underbrace{\widetilde{x}(t+1)}_{=0}+q(t) \cdot \widetilde{x}(t)=0
$$

und $q(t) \neq 0$ folgt $\widetilde{x}(t)=0$, im Widerspruch zur Auswahl von $t$. Also ist auch $\widetilde{x}(n)=0$ für alle $n \in \mathbb{N}_{0}$ mit $n \leqslant\left(n_{0}-1\right)$.

Wir haben gezeigt, dass das Anfangswertproblem (4.1.3) nur die triviale Lösung $\widetilde{x}(n)=0$ für alle $n \in \mathbb{N}_{0}$ besitzt, d.h., es gilt $x_{1}(n)=x_{2}(n)$ für alle $n \in \mathbb{N}_{0}$. Damit ist die Lösung von (4.1.2) eindeutig bestimmt.

\section{Bemerkung und Definition 4.1.3}

Ist $f(n)=0$ für alle $n \in \mathbb{N}_{0}$, so heißt die Gleichung (4.1.1) lineare homogene Differenzengleichung zweiter Ordnung, ansonsten heißt diese lineare inhomogene Differenzengleichung zweiter Ordnung. 


\subsection{Lineare homogene Differenzengleichung zweiter Ordnung}

Wir untersuchen zuerst die homogene Gleichung

$$
x(n+2)+p(n) \cdot x(n+1)+q(n) \cdot x(n)=0
$$

(dabei sind die Funktionen $p, q: \mathbb{N}_{0} \rightarrow \mathbb{R}$ mit $q(n) \neq 0$ für alle $n \in \mathbb{N}_{0}$ gegeben).

\section{Satz 4.2.1}

Seien $x_{1}, x_{2}: \mathbb{N}_{0} \rightarrow \mathbb{R}$ zwei Lösungen von (4.2.1), dann ist

$$
x(n):=c_{1} \cdot x_{1}(n)+c_{2} \cdot x_{2}(n)
$$

ebenfalls eine Lösung von (4.2.1), wobei $c_{1}, c_{2} \in \mathbb{R}$ zwei beliebige Konstanten sind. BeweIs:

Da $x_{1}, x_{2}$ zwei Lösungen von (4.2.1) sind, gilt für alle $n \in \mathbb{N}_{0}$ :

$$
x_{1}(n+2)+p(n) \cdot x_{1}(n+1)+q(n) \cdot x_{1}(n)=0
$$

und

$$
x_{2}(n+2)+p(n) \cdot x_{2}(n+1)+q(n) \cdot x_{2}(n)=0 .
$$

Dann folgt für $x(n):=c_{1} \cdot x_{1}(n)+c_{2} \cdot x_{2}(n)$ mit beliebigen $c_{1}, c_{2} \in \mathbb{R}$ :

$$
\begin{aligned}
x(n+2)+p(n) \cdot x(n+1)+q(n) \cdot x(n) \\
=\left[c_{1} \cdot x_{1}(n+2)+c_{2} \cdot x_{2}(n+2)\right] \\
+p(n) \cdot\left[c_{1} \cdot x_{1}(n+1)+c_{2} \cdot x_{2}(n+1)\right] \\
\quad+q(n) \cdot\left[c_{1} \cdot x_{1}(n)+c_{2} \cdot x_{2}(n)\right] \\
=c_{1} \cdot\left[\begin{array}{l}
\left.x_{1}(n+2)+p(n) \cdot x_{1}(n+1)+q(n) \cdot x_{1}(n)\right] \\
+c_{2} \cdot\left[x_{2}(n+2)+p(n) \cdot x_{2}(n+1)+q(n) \cdot x_{2}(n)\right]
\end{array}\right.
\end{aligned}
$$

$\underset{(4.2 .2 \mathrm{~b})}{\stackrel{(4.2 .2 \mathrm{a})}{=}} 0$ für alle $n \in \mathbb{N}_{0}$.

Nach Definition 4.1.2 ist

$$
x(n)=c_{1} \cdot x_{1}(n)+c_{2} \cdot x_{2}(n)
$$

eine Lösung von (4.2.1) auf $\mathbb{N}_{0}$, für beliebige $c_{1}, c_{2} \in \mathbb{R}$. 


\section{Definition 4.2.2 (Komplexwertige Funktion der ganzzahligen Variablen)}

Sei $\mathbb{I}$ eine nichtleere Teilmenge von $\mathbb{Z}$. Eine Abbildung $w: \mathbb{I} \rightarrow \mathbb{C}$ heißt komplexwertige Funktion der ganzzahligen Variablen.

\section{Bemerkung 4.2.3}

fede komplexwertige Funktion $w: \mathbb{I} \rightarrow \mathbb{C}$ der ganzzahligen Variablen lässt sich in der Form $w=u+\mathrm{i} \cdot v$ darstellen, wobei $u, v$ zwei reellwertige Funktionen der ganzzahligen Variablen sind.

\section{Beispiel 4.2.4}

Seien $r, \varphi \in \mathbb{R}$ mit $r \neq 0$. Dann ist die Funktion $w: \mathbb{Z} \rightarrow \mathbb{C}$ mit

$$
w(n):=(r \cdot(\cos (\varphi)+\mathrm{i} \cdot \sin (\varphi)))^{n}
$$

auf $\mathbb{Z}$ wohldefiniert und es gilt für alle $n \in \mathbb{Z}$ :

$$
\begin{aligned}
(r \cdot(\cos (\varphi)+\mathrm{i} \cdot \sin (\varphi)))^{n} & =r^{n} \cdot(\cos (\varphi)+\mathrm{i} \cdot \sin (\varphi))^{n} \\
& \stackrel{(*)}{=} r^{n} \cdot(\cos (n \varphi)+\mathrm{i} \cdot \sin (n \varphi)) \\
& =r^{n} \cdot \cos (n \varphi)+\mathrm{i} \cdot r^{n} \cdot \sin (n \varphi),
\end{aligned}
$$

wobei wir in $(*)$ den Satz von MoIvre ${ }^{8}$ benutzt haben:

\section{Satz von MoIvre}

$$
(\cos (\varphi)+\mathrm{i} \cdot \sin (\varphi))^{n}=\cos (n \varphi)+\mathrm{i} \cdot \sin (n \varphi) \quad \text { für alle } \varphi \in \mathbb{R} \text { und alle } n \in \mathbb{Z} \text {. }
$$

\section{Satz 4.2.5}

Ist $x(n):=u(n)+\mathrm{i} \cdot v(n)$ mit reellwertigen Funktionen $u, v: \mathbb{N}_{0} \rightarrow \mathbb{R}$ eine komplexwertige Lösung von (4.2.1), dann lösen diese beiden Funktionen ebenfalls diese Differenzengleichung.

\footnotetext{
${ }^{8}$ Abraham de Moivre, 1667-1754
} 
BeweIs:

Da die Funktion $x=u+\mathrm{i} \cdot v$ eine komplexwertige Lösung der Gleichung (4.2.1) ist, gilt für alle $n \in \mathbb{N}_{0}$ (nach Definition 4.1.1):

$$
\begin{aligned}
& 0=x(n+2)+p(n) \cdot x(n+1)+q(n) \cdot x(n) \\
& =[u(n+2)+\mathrm{i} \cdot v(n+2)] \\
& \quad+p(n) \cdot[u(n+1)+\mathrm{i} \cdot v(n+1)] \\
& \quad+q(n) \cdot[u(n)+\mathrm{i} \cdot v(n)] \\
& =[u(n+2)+p(n) \cdot u(n+1)+q(n) \cdot u(n)] \\
& \quad+\mathrm{i} \cdot[v(n+2)+p(n) \cdot v(n+1)+q(n) \cdot v(n)] .
\end{aligned}
$$

Für jedes $n \in \mathbb{N}_{0}$ liefert der Vergleich zweier komplexen Zahlen:

$$
\left\{\begin{array}{l}
u(n+2)+p(n) \cdot u(n+1)+q(n) \cdot u(n)=0, \\
v(n+2)+p(n) \cdot v(n+1)+q(n) \cdot v(n)=0 .
\end{array}\right.
$$

Nach Definition 4.1.1 sind also die Funktionen $u, v$ Lösungen der homogenen Gleichung (4.2.1) auf $\mathbb{N}_{0}$.

\section{Beispiel 4.2.6}

Die komplexwertige Funktion $x: \mathbb{N}_{0} \rightarrow \mathbb{C}$ mit $x(n):=(2 \cdot \mathrm{i})^{n}$ ist eine Lösung der Gleichung

$$
x(n+2)+4 x(n)=0 \quad \text { auf } \quad \mathbb{N}_{0} .
$$

In der Tat

$$
\begin{aligned}
x(n+2)+4 x(n) & =(2 \cdot \mathrm{i})^{n+2}+4 \cdot(2 \cdot \mathrm{i})^{n} \\
& =(2 \cdot \mathrm{i})^{n} \cdot\left((2 \cdot \mathrm{i})^{2}+4\right) \\
& =(2 \cdot \mathrm{i})^{n} \cdot(-4+4)=0
\end{aligned}
$$

für alle $n \in \mathbb{N}_{0}$. Weiter gilt

$$
2 \cdot \mathrm{i}=2 \cdot\left(\cos \left(\frac{\pi}{2}\right)+\mathrm{i} \cdot \sin \left(\frac{\pi}{2}\right)\right)
$$

und nach dem Satz von MoIvre

$$
\begin{aligned}
(2 \cdot \mathrm{i})^{n} & =2^{n} \cdot\left(\cos \left(\frac{\pi}{2}\right)+\mathrm{i} \cdot \sin \left(\frac{\pi}{2}\right)\right)^{n} \\
& =2^{n} \cdot\left(\cos \left(\frac{\pi}{2} \cdot n\right)+\mathrm{i} \cdot \sin \left(\frac{\pi}{2} \cdot n\right)\right) .
\end{aligned}
$$


Für jedes $n \in \mathbb{N}_{0}$ seien $u(n):=2^{n} \cdot \cos \left(\frac{\pi}{2} \cdot n\right)$ und $v(n):=2^{n} \cdot \sin \left(\frac{\pi}{2} \cdot n\right)$. Dann gilt auf $\mathbb{N}_{0}$

$$
\begin{aligned}
u(n+2)+4 u(n) & =2^{n+2} \cdot \cos \left(\frac{\pi}{2} \cdot(n+2)\right)+4 \cdot 2^{n} \cdot \cos \left(\frac{\pi}{2} \cdot n\right) \\
& =2^{n+2} \cdot\left(\cos \left(\frac{\pi}{2} \cdot n+\pi\right)+\cos \left(\frac{\pi}{2} \cdot n\right)\right) \\
& =2^{n+2} \cdot\left(-\cos \left(\frac{\pi}{2} \cdot n\right)+\cos \left(\frac{\pi}{2} \cdot n\right)\right)=0
\end{aligned}
$$

sowie

$$
\begin{aligned}
v(n+2)+4 v(n) & =2^{n+2} \cdot \sin \left(\frac{\pi}{2} \cdot(n+2)\right)+4 \cdot 2^{n} \cdot \sin \left(\frac{\pi}{2} \cdot n\right) \\
& =2^{n+2} \cdot\left(\sin \left(\frac{\pi}{2} \cdot n+\pi\right)+\sin \left(\frac{\pi}{2} \cdot n\right)\right) \\
& =2^{n+2} \cdot\left(-\sin \left(\frac{\pi}{2} \cdot n\right)+\sin \left(\frac{\pi}{2} \cdot n\right)\right)=0
\end{aligned}
$$

D.h., die Funktionen $u, v$ sind reellwertige Lösungen von $x(n+2)+4 x(n)=0$ auf $\mathbb{N}_{0}$.

\section{Definition 4.2.7}

Gegeben seien die Funktionen $x_{1}, x_{2}: \mathbb{N}_{0} \rightarrow \mathbb{R}$. Dann heißt die Determinante

$$
D(n):=\operatorname{det}\left(\begin{array}{cc}
x_{1}(n) & x_{2}(n) \\
x_{1}(n+1) & x_{2}(n+1)
\end{array}\right)=:\left|\begin{array}{cc}
x_{1}(n) & x_{2}(n) \\
x_{1}(n+1) & x_{2}(n+1)
\end{array}\right|
$$

CASORATI-Determinante ${ }^{9}$ dieser Funktionen.

\section{Definition 4.2.8}

Seien $x_{1}$ und $x_{2} z$ wei verschiedene Lösungen von (4.2.1). Dann heißt $\left\{x_{1}, x_{2}\right\}$ heißt Fundamentalsystem von (4.2.1) auf $\mathbb{N}_{0}$, falls für die CASORATI-Determinante dieser Funktionen stets $D(n) \neq 0$ für alle $n \in \mathbb{N}_{0}$ erfüllt ist.

\section{Beispiel 4.2.9}

$$
x(n+2)-x(n)=0, \quad n \in \mathbb{N}_{0} .
$$

1) $x_{1}(n):=1^{n}=1$ und $x_{2}(n):=(-1)^{n}$ sind Lösungen dieser Gleichung auf $\mathbb{N}_{0}$. In der Tat gilt für jedes $n \in \mathbb{N}_{0}$ :

$$
x_{1}(n+2)=1^{n+2}=1, \quad x_{1}(n+2)-x_{1}(n)=1^{n+2}-1^{n}=0
$$

${ }^{9}$ Felice Casorati, $1835-1890$ 
sowie

$$
\begin{aligned}
x_{2}(n+2)=(-1)^{n+2}, \quad x_{2}(n+2)-x_{2}(n) & =(-1)^{n+2}-(-1)^{n} \\
& =(-1)^{n} \cdot(1-1)=0 .
\end{aligned}
$$

2) Nach Definition 4.2.7 gilt für alle $n \in \mathbb{N}_{0}$ :

$$
\begin{aligned}
D(n) & =\left|\begin{array}{cc}
x_{1}(n) & x_{2}(n) \\
x_{1}(n+1) & x_{2}(n+1)
\end{array}\right|=\left|\begin{array}{cc}
1 & (-1)^{n} \\
1 & (-1)^{n+1}
\end{array}\right|=(-1)^{n+1}-(-1)^{n} \\
& =2 \cdot(-1)^{n+1} \neq 0 .
\end{aligned}
$$

Nach Definition 4.2.8 ist $\left\{1,(-1)^{n}\right\}$ ein Fundamentalsystem der Gleichung $x(n+2)-x(n)=0$ auf $\mathbb{N}_{0}$.

\section{Satz 4.2.10}

Seien $x_{1}$ und $x_{2}$ zwei verschiedene Lösungen von (4.2.1). Existiert ein $n_{0} \in \mathbb{N}_{0}$ mit $D\left(n_{0}\right) \neq 0$, so gilt $D(n) \neq 0$ für alle $n \in \mathbb{N}_{0}$.

\section{BEWEIS:}

Da die Funktionen $x_{1}, x_{2}: \mathbb{N}_{0} \rightarrow \mathbb{R}$ zwei verschiedene Lösungen der Gleichung (4.2.1) sind, gilt für jedes $n \in \mathbb{N}_{0}$ und für jedes $k \in\{1,2\}$ :

$$
\begin{aligned}
& x_{k}(n+2)+p(n) \cdot x_{k}(n+1)+q(n) \cdot x_{k}(n)=0 \\
\Leftrightarrow \quad & x_{k}(n+2)=-p(n) \cdot x_{k}(n+1)-q(n) \cdot x_{k}(n) .
\end{aligned}
$$

Wir untersuchen die Funktion $D: \mathbb{N}_{0} \rightarrow \mathbb{R}$, wobei

$$
D(n):=\left|\begin{array}{cc}
x_{1}(n) & x_{2}(n) \\
x_{1}(n+1) & x_{2}(n+1)
\end{array}\right|=x_{1}(n) \cdot x_{2}(n+1)-x_{1}(n+1) \cdot x_{2}(n)
$$

CAsorati-Determinante der Funktionen $x_{1}, x_{2}$ ist.

Weiter gilt für jedes $n \in \mathbb{N}_{0}$ :

$$
\begin{aligned}
D(n+1) & =\left|\begin{array}{ll}
x_{1}(n+1) & x_{2}(n+1) \\
x_{1}(n+2) & x_{2}(n+2)
\end{array}\right| \\
& =x_{1}(n+1) \cdot x_{2}(n+2)-x_{1}(n+2) \cdot x_{2}(n+1) \\
& \stackrel{\text { s.o. }}{=} x_{1}(n+1) \cdot\left[-p(n) \cdot x_{2}(n+1)-q(n) \cdot x_{2}(n)\right] \\
& \quad-\left[-p(n) \cdot x_{1}(n+1)-q(n) \cdot x_{1}(n)\right] \cdot x_{2}(n+1) \\
& =q(n) \cdot\left[x_{1}(n) \cdot x_{2}(n+1)-x_{1}(n+1) \cdot x_{2}(n)\right] \\
& =q(n) \cdot D(n) .
\end{aligned}
$$


Das Anfangswertproblem

$$
\left\{\begin{aligned}
D(n+1) & =q(n) \cdot D(n) \\
D\left(n_{0}\right) & =D_{0} \quad\left(\text { mit } D_{0} \in \mathbb{R}\right)
\end{aligned}\right.
$$

ist auf $\mathbb{N}_{0}$ eindeutig lösbar (vgl. Folgerung 2.2.5) und es gilt für jedes $n \in \mathbb{N}_{0}$ :

$$
D(n)=D\left(n_{0}\right) \cdot \frac{\prod_{k=0}^{n-1} q(n)}{\prod_{k=0}^{n_{0}-1} q(n)}
$$

wobei wir stets $q(n) \neq 0$ für alle $n \in \mathbb{N}_{0}$ haben.

Somit folgt: Ist $D\left(n_{0}\right) \neq 0$, so gilt auch $D(n) \neq 0$ für alle $n \in \mathbb{N}_{0}$.

\section{Satz und Definition 4.2.11}

Allgemeine Lösung der linearen homogenen Differenzengleichung 2. Ordnung.

Sei $\left\{x_{1}, x_{2}\right\}$ ein Fundamentalsystem von (4.2.1) auf $\mathbb{N}_{0}$, dann lässt sich jede Lösung $x: \mathbb{N}_{0} \rightarrow \mathbb{R}$ von (4.2.1) in der Form

$$
x(n)=c_{1} \cdot x_{1}(n)+c_{2} \cdot x_{2}(n)
$$

darstellen, wobei die Koeffizienten $c_{1}, c_{2} \in \mathbb{R}$ eindeutig bestimmt sind.

Lässt man in $c_{1} \cdot x_{1}(n)+c_{2} \cdot x_{2}(n)$ die Koeffizienten $c_{1}, c_{2}$ unabhängig von einander die Menge der reellen Zahlen durchlaufen, so erhält man alle Lösungen der Gleichung (4.2.1). Man sagt: Durch $x(n)=c_{1} \cdot x_{1}(n)+c_{2} \cdot x_{2}(n)$ ist die allgemeine Lösung der linearen homogenen Differenzengleichung 2. Ordnung

$$
x(n+2)+p(n) \cdot x(n+1)+q(n) \cdot x(n)=0
$$

auf $\mathbb{N}_{0}$ gegeben.

BEWEIS:

Da $\left\{x_{1}, x_{2}\right\}$ ein Fundamentalsystem von (4.2.1) ist, ist nach Definition 4.2.8 und Satz 4.2.1 jede Funktion $x: \mathbb{N}_{0} \rightarrow \mathbb{R}$ mit

$$
x(n):=c_{1} \cdot x_{1}(n)+c_{2} \cdot x_{2}(n)
$$

immer eine Lösung von (4.2.1) für beliebige $c_{1}, c_{2} \in \mathbb{R}$. 
Sei $\widetilde{x}: \mathbb{N}_{0} \rightarrow \mathbb{R}$ eine beliebige Lösung von (4.2.1). Wir zeigen: Es existieren $c_{1}, c_{2} \in \mathbb{R}$, sodass auf $\mathbb{N}_{0}$ gilt:

$$
\widetilde{x}(n)=c_{1} \cdot x_{1}(n)+c_{2} \cdot x_{2}(n)
$$

In der Tat: Wir nehmen ein beliebiges $n_{0} \in \mathbb{N}_{0}$ und bestimmen die Werte $\widetilde{x}\left(n_{0}\right)$ und $\widetilde{x}\left(n_{0}+1\right)$. Wir suchen $c_{1}, c_{2} \in \mathbb{R}$ mit:

$$
\left\{\begin{aligned}
c_{1} \cdot x_{1}\left(n_{0}\right)+c_{2} \cdot x_{2}\left(n_{0}\right) & =\widetilde{x}\left(n_{0}\right), \\
c_{1} \cdot x_{1}\left(n_{0}+1\right)+c_{2} \cdot x_{2}\left(n_{0}+1\right) & =\widetilde{x}\left(n_{0}+1\right) .
\end{aligned}\right.
$$

Weiter gilt

$$
\triangle:=\left|\begin{array}{cc}
x_{1}\left(n_{0}\right) & x_{2}\left(n_{0}\right) \\
x_{1}\left(n_{0}+1\right) & x_{2}\left(n_{0}+1\right)
\end{array}\right|=D\left(n_{0}\right) \neq 0,
$$

da $\left\{x_{1}, x_{2}\right\}$ ein Fundamentalsystem von (4.2.1) ist. Dann ist das LGS (*) eindeutig für $c_{1}, c_{2}$ lösbar. Sei $\left\{\widetilde{c_{1}}, \widetilde{c_{2}}\right\}$ diese eindeutige Lösung des LGS $(*)$. Wir untersuchen die Funktion $x: \mathbb{N}_{0} \rightarrow \mathbb{R}$ mit

$$
x(n):=\widetilde{c_{1}} \cdot x_{1}(n)+\widetilde{c_{2}} \cdot x_{2}(n) .
$$

Nach Satz 4.2.1 ist $x$ eine Lösung von (4.2.1) auf $\mathbb{N}_{0}$ und

$$
x\left(n_{0}\right)=\widetilde{c_{1}} \cdot x_{1}\left(n_{0}\right)+\widetilde{c_{2}} \cdot x_{2}\left(n_{0}\right)=\widetilde{x}\left(n_{0}\right),
$$

sowie

$$
x\left(n_{0}+1\right)=\widetilde{c_{1}} \cdot x_{1}\left(n_{0}+1\right)+\widetilde{c_{2}} \cdot x_{2}\left(n_{0}+1\right)=\widetilde{x}\left(n_{0}+1\right) .
$$

Wegen der Eindeutigkeit der Lösung des Anfangswertproblems (Satz 4.1.2) gilt für alle $n \in \mathbb{N}_{0}$ :

$$
x(n)=\widetilde{x}(n)
$$

und

$$
\widetilde{x}(n)=\widetilde{c_{1}} \cdot x_{1}(n)+\widetilde{c_{2}} \cdot x_{2}(n)
$$

Dann ist jede Lösung von (4.2.1) in der Form $c_{1} \cdot x_{1}(n)+c_{2} \cdot x_{2}(n)$ mit $c_{1}, c_{2} \in \mathbb{R}$ darstellbar. 


\section{Beispiel 4.2.12}

$$
x(n+2)+x(n)=0, \quad n \in \mathbb{N}_{0} .
$$

1) $x_{1}(n):=\cos \left(\frac{\pi}{2} \cdot n\right)$ und $x_{2}(n):=\sin \left(\frac{\pi}{2} \cdot n\right)$ sind die Lösungen der Gleichung auf $\mathbb{N}_{0}$. In der Tat gilt für jedes $n \in \mathbb{N}_{0}$ :

$$
\begin{aligned}
x_{1}(n+2)+x_{1}(n) & =\cos \left(\frac{\pi}{2} \cdot(n+2)\right)+\cos \left(\frac{\pi}{2} \cdot n\right) \\
& =\cos \left(\frac{\pi}{2} \cdot n+\pi\right)+\cos \left(\frac{\pi}{2} \cdot n\right) \\
& =-\cos \left(\frac{\pi}{2} \cdot n\right)+\cos \left(\frac{\pi}{2} \cdot n\right)=0,
\end{aligned}
$$

und

$$
\begin{aligned}
x_{2}(n+2)+x_{2}(n) & =\sin \left(\frac{\pi}{2} \cdot(n+2)\right)+\sin \left(\frac{\pi}{2} \cdot n\right) \\
& =\sin \left(\frac{\pi}{2} \cdot n+\pi\right)+\sin \left(\frac{\pi}{2} \cdot n\right) \\
& =-\sin \left(\frac{\pi}{2} \cdot n\right)+\sin \left(\frac{\pi}{2} \cdot n\right)=0 .
\end{aligned}
$$

2) Nach Definition 4.2.7 gilt für alle $n \in \mathbb{N}_{0}$ :

$$
\begin{aligned}
D(n) & =\left|\begin{array}{cc}
x_{1}(n) & x_{2}(n) \\
x_{1}(n+1) & x_{2}(n+1)
\end{array}\right| \\
& =\left|\begin{array}{cc}
\cos \left(\frac{\pi}{2} \cdot n\right) & \sin \left(\frac{\pi}{2} \cdot n\right) \\
\cos \left(\frac{\pi}{2} \cdot(n+1)\right) & \sin \left(\frac{\pi}{2} \cdot(n+1)\right)
\end{array}\right| \\
& =\sin \left(\frac{\pi}{2} \cdot n+\frac{\pi}{2}\right) \cdot \cos \left(\frac{\pi}{2} \cdot n\right)-\cos \left(\frac{\pi}{2} \cdot n+\frac{\pi}{2}\right) \cdot \sin \left(\frac{\pi}{2} \cdot n\right) \\
& =\sin \left(\frac{\pi}{2}\right)=1 \neq 0 .
\end{aligned}
$$

Nach Definition 4.2.8 ist somit $\left\{\cos \left(\frac{\pi}{2} \cdot n\right), \sin \left(\frac{\pi}{2} \cdot n\right)\right\}$ ein Fundamentalsystem von $x(n+2)+x(n)=0$ auf $\mathbb{N}_{0}$.

3) Die allgemeine Lösung der Gleichung $x(n+2)+x(n)=0$ auf $\mathbb{N}_{0}$ lautet also nach Satz 4.2.11:

$$
x(n)=c_{1} \cdot \cos \left(\frac{\pi}{2} \cdot n\right)+c_{2} \cdot \sin \left(\frac{\pi}{2} \cdot n\right)
$$

mit beliebigen $c_{1}, c_{2} \in \mathbb{R}$. 


\section{Satz 4.2.13}

Sei $x_{1}$ eine Lösung von (4.2.1), sodass $x_{1}(n) \neq 0$ für alle $n \in \mathbb{N}_{0}$. Dann kann man eine zweite Lösung $x_{2}$ der Gleichung (4.2.1) bestimmen, sodass $\left\{x_{1}, x_{2}\right\}$ ein Fundamentalsystem von (4.2.1) bildet.

BEwEIS:

Wir suchen die Lösung $x_{2}$ in der Form $x_{2}(n)=x_{1}(n) \cdot u(n)$, wobei $u: \mathbb{N}_{0} \rightarrow$ $\mathbb{R}$ eine unbekannte Funktion ist. In die Differenzengleichung (4.2.1) eingesetzt ergibt:

$$
\begin{aligned}
x_{1}(n+2) \cdot u(n+2)+p(n) \cdot x_{1}(n+1) & u(n+1) \\
& +q(n) \cdot x_{1}(n) \cdot u(n)=0 .
\end{aligned}
$$

Da $x_{1}$ eine Lösung von (4.2.1) ist, gilt $p(n) \cdot x_{1}(n+1)=-x_{1}(n+2)-q(n) \cdot x_{1}(n)$ und wir erhalten

$$
\begin{array}{r}
x_{1}(n+2) \cdot u(n+2)+\left(-x_{1}(n+2)-q(n) \cdot x_{1}(n)\right) \cdot u(n+1) \\
+q(n) \cdot x_{1}(n) \cdot u(n)=0 \\
\Leftrightarrow \quad x_{1}(n+2) \cdot(u(n+2)-u(n+1)) \\
+q(n) \cdot x_{1}(n) \cdot(u(n)-u(n+1))=0
\end{array}
$$

M.a.W. genügt die Funktion $w: \mathbb{N}_{0} \rightarrow \mathbb{R}$ mit $w(n):=u(n+1)-u(n)$ der folgenden linearen homogenen Differenzengleichung erster Ordnung auf $\mathbb{N}_{0}$ :

$$
w(n+1)-q(n) \cdot \frac{x_{1}(n)}{x_{1}(n+2)} \cdot w(n)=0 .
$$

Nach Bemerkung 2.2.2 ist dann

$$
w(n):=\prod_{k=0}^{n-1} q(k) \cdot \frac{x_{1}(k)}{x_{1}(k+2)}=\frac{x_{1}(0) \cdot x_{1}(1)}{x_{1}(n) \cdot x_{1}(n+1)} \cdot \prod_{k=0}^{n-1} q(k)
$$

eine Lösung von (4.2.3) auf $\mathbb{N}_{0}$. Weiterhin bekommt man $u$ als eine Lösung der Gleichung $u(n+1)-u(n)=w(n) \quad \operatorname{durch} u(n):=\sum_{k=0}^{n-1} w(k)$. Mit dieser Funktion $u$ ist also $x_{2}(n)=x_{1}(n) \cdot u(n)$ eine (weitere) Lösung der Gleichung (4.2.1) auf $\mathbb{N}_{0}$. 
Nach Definition 4.2.7 erhalten wir ferner für alle $n \in \mathbb{N}_{0}$ :

$$
\begin{aligned}
D(n) & =\left|\begin{array}{cc}
x_{1}(n) & x_{2}(n) \\
x_{1}(n+1) & x_{2}(n+1)
\end{array}\right|=\left|\begin{array}{cc}
x_{1}(n) & x_{1}(n) \cdot u(n) \\
x_{1}(n+1) & x_{1}(n+1) \cdot u(n+1)
\end{array}\right| \\
& =x_{1}(n) \cdot x_{1}(n+1) \cdot(u(n+1)-u(n))=x_{1}(n) \cdot x_{1}(n+1) \cdot w(n) \\
& =x_{1}(0) \cdot x_{1}(1) \cdot \prod_{k=0}^{n-1} q(k) \neq 0 .
\end{aligned}
$$

Somit ist $\left\{x_{1}, x_{2}\right\}$ nach Definition 4.2.8 ein Fundamentalsystem der homogenen Gleichung (4.2.1) auf $\mathbb{N}_{0}$.

\section{Beispiel 4.2.14}

$$
x(n+2)-4 x(n+1)+4 x(n)=0, \quad n \in \mathbb{N}_{0} .
$$

1) Wir suchen zunächst eine komplexwertige Lösung $x_{1}$ in der Form $x_{1}(n):=\lambda^{n}$ mit $\lambda \in \mathbb{C} \backslash\{0\}$. Dann gilt:

$$
\begin{gathered}
x(n+2)-4 x(n+1)+4 x(n)=0 \quad \Leftrightarrow \quad \lambda^{n+2}-4 \lambda^{n+1}+4 \lambda^{n}=0 \\
\Leftrightarrow \quad \lambda^{n} \cdot\left(\lambda^{2}-4 \lambda+4\right)=0 \quad \underset{\lambda \neq 0}{\Longleftrightarrow} \quad \lambda^{2}-4 \lambda+4=0 \quad \Leftrightarrow \quad \lambda=2 .
\end{gathered}
$$

Also ist $x_{1}(n)=2^{n}$ eine reellwertige Lösung der Gleichung (4.2.4) auf $\mathbb{N}_{0}$.

2) Wir suchen eine weitere Lösung $x_{2}$ in der Form $x_{2}(n):=x_{1}(n) \cdot u(n)$, wobei $u: \mathbb{N}_{0} \rightarrow \mathbb{R}$ eine neue unbekannte Funktion ist. Für jedes $n \in \mathbb{N}_{0}$ gilt:

$$
x_{2}(n+2)-4 x_{2}(n+1)+4 x_{2}(n)=0
$$

und eingesetzt

$$
x_{1}(n+2) \cdot u(n+2)-4 x_{1}(n+1) \cdot u(n+1)+4 x_{1}(n) \cdot u(n)=0 .
$$

Da $x_{1}$ eine Lösung ist, gilt $4 x_{1}(n+1)=x_{1}(n+2)+4 x_{1}(n)$, sodass wir erhalten

$$
\begin{array}{r}
x_{1}(n+2) \cdot u(n+2)-\left(x_{1}(n+2)+4 x_{1}(n)\right) \cdot u(n+1)+4 x_{1}(n) \cdot u(n)=0 \\
\Leftrightarrow \quad x_{1}(n+2) \cdot(u(n+2)-u(n+1))-4 x_{1}(n) \cdot(u(n+1)-u(n))=0
\end{array}
$$


M.a.W. genügt die Funktion $w: \mathbb{N}_{0} \rightarrow \mathbb{R}$ mit $w(n):=u(n+1)-u(n)$ der Differenzengleichung

$$
x_{1}(n+2) \cdot w(n+1)-4 x_{1}(n) \cdot w(n)=0
$$

und wir erhalten mit $x_{1}(n)=2^{n}$ für alle $n \in \mathbb{N}_{0}$ :

$$
\begin{aligned}
2^{n+2} \cdot w(n+1)-4 \cdot 2^{n} \cdot w(n) & =0 \\
\underset{2^{n+2} \neq 0}{\Leftrightarrow} & w(n+1)-w(n)=0 \quad \Leftrightarrow \quad w(n+1)=w(n) .
\end{aligned}
$$

Wir wählen (z.B.) $w(n)=1$ für alle $n \in \mathbb{N}_{0}$. Dann gilt für alle $n \in \mathbb{N}_{0}$ :

$$
u(n+1)-u(n)=1
$$

Wir nehmen (z.B.) $u(n)=n$, woraus $x_{2}(n)=2^{n} \cdot n$ für alle $n \in \mathbb{N}_{0}$ folgt. $x_{2}(n)=2^{n} \cdot n$ ist auch eine Lösung der Gleichung (4.2.4) auf $\mathbb{N}_{0}$.

3) Nach Definition 4.2.7 gilt für alle $n \in \mathbb{N}_{0}$ :

$$
\begin{aligned}
D(n) & =\left|\begin{array}{cc}
x_{1}(n) & x_{2}(n) \\
x_{1}(n+1) & x_{2}(n+1)
\end{array}\right|=\left|\begin{array}{cc}
2^{n} & 2^{n} \cdot n \\
2^{n+1} & 2^{n+1} \cdot(n+1)
\end{array}\right| \\
& =2^{2 n+1} \neq 0 .
\end{aligned}
$$

Nach Definition 4.2.8 ist somit $\left\{2^{n}, 2^{n} \cdot n\right\}$ ein Fundamentalsystem der homogenen Gleichung (4.2.4) auf $\mathbb{N}_{0}$.

4) Die allgemeine Lösung der Gleichung (4.2.4) auf $\mathbb{N}_{0}$ lautet nach Satz 4.2.11:

$$
x(n)=c_{1} \cdot 2^{n}+c_{2} \cdot n \cdot 2^{n}=2^{n} \cdot\left(c_{1}+c_{2} \cdot n\right)
$$

mit beliebigen $c_{1}, c_{2} \in \mathbb{R}$.

\section{Satz 4.2.15}

Gegeben seien die Funktionen $p, q: \mathbb{N}_{0} \rightarrow \mathbb{R}$, wobei $q(n) \neq 0$ für alle $n \in \mathbb{N}_{0}$ ist. Dann besitzt die lineare homogene Differenzengleichung 2. Ordnung

$$
x(n+2)+p(n) \cdot x(n+1)+q(n) \cdot x(n)=0
$$

ein Fundamentalsystem auf $\mathbb{N}_{0}$. 


\section{BEWEIS:}

Wir finden zwei (spezielle) Lösungen $x_{1}$ und $x_{2}$ der homogenen Differenzengleichung (4.2.1) mit folgenden Anfangsbedingungen:

$$
\left\{\begin{array} { l } 
{ x _ { 1 } ( 0 ) = 1 } \\
{ x _ { 1 } ( 1 ) = 0 }
\end{array} \quad \text { bzw. } \quad \left\{\begin{array}{l}
x_{2}(0)=0 \\
x_{2}(1)=1
\end{array}\right.\right.
$$

Nach Satz 4.1.2 sind die Funktionen $x_{1}, x_{2}: \mathbb{N}_{0} \rightarrow \mathbb{R}$ eindeutig bestimmt. An der Stelle 0 erhalten wir für die CASORATI-Determinante dieser Funktionen:

$$
D(0):=\left|\begin{array}{ll}
x_{1}(0) & x_{2}(0) \\
x_{1}(1) & x_{2}(1)
\end{array}\right|=\left|\begin{array}{ll}
1 & 0 \\
0 & 1
\end{array}\right|=1 \neq 0 .
$$

Nach Satz 4.2.10 ist $D(n) \neq 0$ für alle $n \in \mathbb{N}_{0}$ und nach Definition 4.2.8 ist $\left\{x_{1}, x_{2}\right\}$ ein Fundamentalsystem von (4.2.1) auf $\mathbb{N}_{0}$.

\subsection{Lineare homogene Differenzengleichung zwei- ter Ordnung mit konstanten Koeffizienten}

\section{Definition 4.3.1}

Gegeben seien $p, q \in \mathbb{R}$ mit $q \neq 0$. Gesucht ist eine Funktion $x: \mathbb{N}_{0} \rightarrow \mathbb{R}$, sodass für alle $n \in \mathbb{N}_{0}$ gilt

$$
x(n+2)+p \cdot x(n+1)+q \cdot x(n)=0 .
$$

Die Gleichung (4.3.1) heißt lineare homogene Differenzengleichung zweiter Ordnung mit konstanten Koeffizienten. Die Funktion $x: \mathbb{N}_{0} \rightarrow \mathbb{R}$ heißt Lösung der Gleichung (4.3.1) auf $\mathbb{N}_{0}$.

Eine Funktion $x: \mathbb{N}_{0} \rightarrow \mathbb{C}$, die (4.3.1) erfüllt, heißt komplexwertige Lösung der Gleichung (4.3.1) auf $\mathbb{N}_{0}$. 


\section{Bemerkung 4.3.2}

Wir suchen Lösungen der Gleichung (4.3.1) in der Form

$$
x_{1}(n):=\lambda^{n}
$$

mit $\lambda \in \mathbb{C} \backslash\{0\}$. In Gleichung (4.3.1) eingesetzt:

$$
\lambda^{n+2}+p \cdot \lambda^{n+1}+q \cdot \lambda^{n}=0,
$$

liefert unter Berücksichtigung, dass $\lambda \neq 0$ ist:

$$
\begin{gathered}
\lambda^{2}+p \cdot \lambda+q=0, \\
\Leftrightarrow \lambda=-\frac{p}{2} \pm \sqrt{\left(\frac{p}{2}\right)^{2}-q}
\end{gathered}
$$

Beachte: $D a q \neq 0$ ist, ist $\lambda=0$ keine Lösung von (4.3.1c).

Gleichung (4.3.1c) heißt charakteristische Gleichung der Differenzengleichung (4.3.1) und $D=\left(\frac{p}{2}\right)^{2}-q$ die Diskriminante der charakteristischen Gleichung (4.3.1c).

Es gilt: Die (komplexwertige) Funktion $x: \mathbb{N}_{0} \rightarrow \mathbb{C}$ mit $x(n):=\lambda^{n}$ und $\lambda \in \mathbb{C} \backslash\{0\}$ ist genau dann eine (komplexwertige) Lösung von (4.3.1) auf $\mathbb{N}_{0}$, wenn $\lambda$ eine (komplexe) Lösung der zugehörigen charakteristischen Gleichung (4.3.1c) ist.

$\underline{\text { I. Fall }}\left(\frac{p}{2}\right)^{2}-q>0$

Seien $\lambda_{1}:=-\frac{p}{2}+\sqrt{\left(\frac{p}{2}\right)^{2}-q}, \quad \lambda_{2}:=-\frac{p}{2}-\sqrt{\left(\frac{p}{2}\right)^{2}-q}$.

Dann sind $\left\{\lambda_{1}, \lambda_{2}\right\}$ zwei verschiedene reelle Lösungen der charakteristischen Gleichung (4.3.1c).

Die reellwertigen Funktionen $x_{1}, x_{2}: \mathbb{N}_{0} \rightarrow \mathbb{R}$ mit $x_{1}(n):=\lambda_{1}^{n}$ und $x_{2}(n):=\lambda_{2}^{n}$ bilden ein Fundamentalsystem von (4.3.1) auf $\mathbb{N}_{0}$. In der Tat:

Die Funktionen $x_{1}, x_{2}$ sind Lösungen von (4.3.1) und für alle $n \in \mathbb{N}_{0}$ gilt:

$$
\begin{aligned}
D(n) & =\left|\begin{array}{cc}
x_{1}(n) & x_{2}(n) \\
x_{1}(n+1) & x_{2}(n+1)
\end{array}\right|=\left|\begin{array}{cc}
\lambda_{1}^{n} & \lambda_{2}^{n} \\
\lambda_{1}^{n+1} & \lambda_{2}^{n+1}
\end{array}\right| \\
& =\left(\lambda_{1} \cdot \lambda_{2}\right)^{n} \cdot\left(\lambda_{2}-\lambda_{1}\right) \neq 0,
\end{aligned}
$$

denn $\lambda_{1} \neq \lambda_{2}$ und $\lambda=0$ ist keine Lösung von (4.3.1c). 


\section{Folgerung 4.3.3}

Nach Satz 4.2.11 ist die Funktion $x: \mathbb{N}_{0} \rightarrow \mathbb{R}$ mit

$$
x(n):=c_{1} \cdot \lambda_{1}^{n}+c_{2} \cdot \lambda_{2}^{n}
$$

und beliebigen $c_{1}, c_{2} \in \mathbb{R}$ die allgemeine Lösung von (4.3.1) im Fall $\left(\frac{p}{2}\right)^{2}-q>0$.

\section{Beispiel 4.3.4}

$$
x(n+2)-2 x(n+1)-3 x(n)=0, \quad n \in \mathbb{N}_{0} .
$$

Die entsprechende charakteristische Gleichung ist

$$
\lambda^{2}-2 \lambda-3=0 \Leftrightarrow \lambda=-1 \quad \text { oder } \quad \lambda=3 .
$$

Seien $\lambda_{1}:=-1$ und $\lambda_{2}:=3$. Dann bilden die Funktionen $x_{1}, x_{2}: \mathbb{N}_{0} \rightarrow \mathbb{R}$ mit

$$
x_{1}(n):=(-1)^{n} \quad \text { und } \quad x_{2}(n):=3^{n}
$$

ein Fundamentalsystem der Gleichung $x(n+2)-2 x(n+1)-3 x(n)=0$ auf $\mathbb{N}_{0}$. Nach Folgerung 4.3.3 ist

$$
x(n)=c_{1} \cdot(-1)^{n}+c_{2} \cdot 3^{n}
$$

mit beliebigen $c_{1}, c_{2} \in \mathbb{R}$ die allgemeine Lösung dieser Gleichung auf $\mathbb{N}_{0}$.

II. Fall $\left(\frac{p}{2}\right)^{2}-q=0$

Die reelle Zahl $\lambda:=-\frac{p}{2}$ ist eine Lösung der charakteristischen Gleichung (4.3.1c) (mit Vielfachheit $r=2$ ). Beachte: da $q \neq 0$ ist, ist hier $p \neq 0$.

Die reellwertige Funktion $x_{1}: \mathbb{N}_{0} \rightarrow \mathbb{R}$ mit $x_{1}(n):=\lambda^{n}$ und $\lambda=-\frac{p}{2}$ ist eine Lösung von (4.3.1). Wir suchen eine weitere Lösung $x_{2}: \mathbb{N}_{0} \rightarrow \mathbb{R}$ in der Form

$$
x_{2}(n):=\lambda^{n} \cdot u(n),
$$

wobei $u: \mathbb{N}_{0} \rightarrow \mathbb{R}$ eine neue unbekannte Funktion ist. Für jedes $n \in \mathbb{N}_{0}$ gilt:

$$
\begin{aligned}
& x_{2}(n+2)+p \cdot x_{2}(n+1)+q \cdot x_{2}(n)=0 \\
& \Longleftrightarrow \quad \lambda^{n+2} \cdot u(n+2)+p \cdot \lambda^{n+1} \cdot u(n+1)+q \cdot \lambda^{n} \cdot u(n)=0 \\
& \underset{\lambda \neq 0}{\Longleftrightarrow} \quad \lambda^{2} \cdot u(n+2)+p \cdot \lambda \cdot u(n+1)+q \cdot u(n)=0 .
\end{aligned}
$$


Da $\lambda$ eine Lösung der charakteristischen Gleichung ist, gilt auch

$$
\lambda^{2} \cdot u(n)+p \cdot \lambda \cdot u(n)+q \cdot u(n)=0 .
$$

Eine Subtraktion der Gleichungen $(*)$ und $(* *)$ liefert:

$$
\begin{aligned}
& \lambda^{2} \cdot(u(n+2)-u(n))+p \cdot \lambda \cdot(u(n+1)-u(n))=0 \\
& \underset{\lambda=-\frac{p}{2}}{\Longleftrightarrow} \quad \frac{p^{2}}{4} \cdot(u(n+2)-u(n))-\frac{p^{2}}{2} \cdot(u(n+1)-u(n))=0 \\
& \Longleftrightarrow \quad(u(n+2)-u(n))-2 \cdot(u(n+1)-u(n))=0 \\
& \Longleftrightarrow \quad(u(n+2)-u(n+1))-(u(n+1)-u(n))=0 .
\end{aligned}
$$

Für alle $n \in \mathbb{N}_{0}$ sei weiter $w(n):=u(n+1)-u(n)$.

Dann gilt $w(n+1)=u(n+2)-u(n+1)$ und

$$
(u(n+2)-u(n+1))-(u(n+1)-u(n))=0 \quad \Leftrightarrow \quad w(n+1)-w(n)=0 .
$$

Für alle $n \in \mathbb{N}_{0}$ nehmen wir $w(n)=1$.

Dann ist

$$
u(n+1)-u(n)=1 .
$$

Wir nehmen $u(n)=n$ für alle $n \in \mathbb{N}_{0}$, woraus

$$
x_{2}(n):=\lambda^{n} \cdot n
$$

folgt.

Die Funktionen $x_{1}, x_{2}: \mathbb{N}_{0} \rightarrow \mathbb{R}$ mit $x_{1}(n):=\lambda^{n}$ und $x_{2}(n):=n \cdot \lambda^{n}$ (wobei $\lambda=-\frac{p}{2}$ ist ) bilden ein Fundamentalsystem von (4.3.1) auf $\mathbb{N}_{0}$. In der Tat gilt für alle $n \in \mathbb{N}_{0}$ :

$$
\begin{aligned}
D(n) & =\left|\begin{array}{cc}
x_{1}(n) & x_{2}(n) \\
x_{1}(n+1) & x_{2}(n+1)
\end{array}\right|=\left|\begin{array}{cc}
\lambda^{n} & n \cdot \lambda^{n} \\
\lambda^{n+1} & (n+1) \cdot \lambda^{n+1}
\end{array}\right| \\
& =\lambda^{2 \cdot n+1} \neq 0,
\end{aligned}
$$

da $\lambda \neq 0$ ist. 


\section{Folgerung 4.3.5}

Nach Satz 4.2.11 ist die Funktion $x: \mathbb{N}_{0} \rightarrow \mathbb{R}$ mit

$$
x(n):=c_{1} \cdot \lambda^{n}+c_{2} \cdot n \cdot \lambda^{n}=\lambda^{n} \cdot\left(c_{1}+c_{2} \cdot n\right)
$$

und beliebigen $c_{1}, c_{2} \in \mathbb{R}$ die allgemeine Lösung von (4.3.1) im Fall $\left(\frac{p}{2}\right)^{2}-q=0$.

\section{Beispiel 4.3.6}

$$
x(n+2)+6 x(n+1)+9 x(n)=0, \quad n \in \mathbb{N}_{0} .
$$

Die entsprechende charakteristische Gleichung ist

$$
\lambda^{2}+6 \lambda+9=0 \quad \Leftrightarrow \quad \lambda=-3 \quad \text { (mit Vielfachheit } r=2 \text { ). }
$$

Dann bilden die Funktionen $x_{1}, x_{2}: \mathbb{N}_{0} \rightarrow \mathbb{R}$ mit

$$
x_{1}:=(-3)^{n} \quad \text { und } \quad x_{2}:=n \cdot(-3)^{n}
$$

ein Fundamentalsystem der Gleichung $x(n+2)+6 x(n+1)+9 x(n)=0$ auf $\mathbb{N}_{0}$. Nach Folgerung 4.3.5 ist

$$
x(n)=(-3)^{n} \cdot\left(c_{1}+c_{2} \cdot n\right)
$$

mit beliebigen $c_{1}, c_{2} \in \mathbb{R}$ die allgemeine Lösung dieser Gleichung auf $\mathbb{N}_{0}$.

$\underline{\text { III. Fall }}\left(\frac{p}{2}\right)^{2}-q<0 \quad \Leftrightarrow \quad q-\left(\frac{p}{2}\right)^{2}>0$

Beachte: Hier ist $q>0$.

Wegen

$$
\begin{aligned}
\lambda & =-\frac{p}{2} \pm \sqrt{\left(\frac{p}{2}\right)^{2}-q}=-\frac{p}{2} \pm \sqrt{-\left(q-\left(\frac{p}{2}\right)^{2}\right)} \\
& =-\frac{p}{2} \pm \mathrm{i} \cdot \sqrt{q-\left(\frac{p}{2}\right)^{2}}
\end{aligned}
$$

sind $\lambda=\alpha \pm \mathrm{i} \cdot \beta$ mit $\alpha:=-\frac{p}{2}$ und $\beta:=\sqrt{q-\left(\frac{p}{2}\right)^{2}}$ zwei verschiedene komplexe Lösungen der charakteristischen Gleichung (4.3.1c). Die komplexwertigen Funktionen $x_{1}^{\star}, x_{2}^{\star}: \mathbb{N}_{0} \rightarrow \mathbb{C}$ mit

$$
x_{1}^{\star}(n):=(\alpha+\mathrm{i} \cdot \beta)^{n} \quad \text { und } \quad x_{2}^{\star}(n):=(\alpha-\mathrm{i} \cdot \beta)^{n}
$$

sind Lösungen der Gleichung (4.3.1). 
Weiter gilt

$$
\alpha+\mathrm{i} \cdot \beta \underset{\beta \neq 0}{=} \sqrt{\alpha^{2}+\beta^{2}} \cdot\left(\frac{\alpha}{\sqrt{\alpha^{2}+\beta^{2}}}+\mathrm{i} \cdot \frac{\beta}{\sqrt{\alpha^{2}+\beta^{2}}}\right) .
$$

Mit $\alpha^{2}+\beta^{2}=q>0$ folgt:

$$
\alpha+\mathrm{i} \cdot \beta=\sqrt{q} \cdot(\cos (\varphi)+\mathrm{i} \cdot \sin (\varphi))
$$

wobei das Argument $\varphi$ durch die Bedingungen

$$
\left\{\begin{array}{l}
\cos (\varphi)=\frac{\alpha}{\sqrt{\alpha^{2}+\beta^{2}}} \\
\sin (\varphi)=\frac{\beta}{\sqrt{\alpha^{2}+\beta^{2}}} \\
\varphi \in[0 ; 2 \pi)
\end{array}\right.
$$

eindeutig bestimmt ist. Da bei uns $\beta>0$ ist, gilt daher

$$
\varphi=\arccos \left(\frac{\alpha}{\sqrt{\alpha^{2}+\beta^{2}}}\right)=\arccos \left(-\frac{p}{2 \sqrt{q}}\right) \in(0 ; \pi) .
$$

Nach dem Satz von MoIvre bekommt man für jedes $n \in \mathbb{N}_{0}$

$$
(\alpha+\mathrm{i} \cdot \beta)^{n}=(q)^{\frac{n}{2}} \cdot(\cos (n \cdot \varphi)+\mathrm{i} \cdot \sin (n \cdot \varphi))
$$

und

$$
\begin{aligned}
(\alpha-\mathrm{i} \cdot \beta)^{n} & =(\overline{\alpha+\mathrm{i} \cdot \beta})^{n}=\overline{(\alpha+\mathrm{i} \cdot \beta)^{n}} \\
& =(q)^{\frac{n}{2}} \cdot(\cos (n \cdot \varphi)-\mathrm{i} \cdot \sin (n \cdot \varphi)) .
\end{aligned}
$$

Also haben wir für jedes $n \in \mathbb{N}_{0}$ :

$$
x_{1}^{\star}(n)=(q)^{\frac{n}{2}} \cdot(\cos (n \cdot \varphi)+\mathrm{i} \cdot \sin (n \cdot \varphi))
$$

sowie

$$
x_{2}^{\star}(n)=(q)^{\frac{n}{2}} \cdot(\cos (n \cdot \varphi)-\mathrm{i} \cdot \sin (n \cdot \varphi)) .
$$

Nach Satz 4.2.5 sind somit auch $\operatorname{Re}\left(x_{1}^{\star}(n)\right), \operatorname{Im}\left(x_{1}^{\star}(n)\right), \operatorname{Re}\left(x_{2}^{\star}(n)\right)$ und $\operatorname{Im}\left(x_{2}^{\star}(n)\right)$ Lösungen der Gleichung (4.3.1). 
Wir wählen (z.B.)

$$
x_{1}(n):=\operatorname{Re}\left(x_{1}^{\star}(n)\right)=(q)^{\frac{n}{2}} \cdot \cos (n \cdot \varphi)
$$

und

$$
x_{2}(n):=\operatorname{Im}\left(x_{1}^{\star}(n)\right)=(q)^{\frac{n}{2}} \cdot \sin (n \cdot \varphi) .
$$

Dann bilden die Funktionen $x_{1}, x_{2}: \mathbb{N}_{0} \rightarrow \mathbb{R}$ ein Fundamentalsystem von (4.3.1) auf $\mathbb{N}_{0}$. In der Tat gilt für jedes $n \in \mathbb{N}_{0}$ :

$$
\begin{aligned}
D(n) & =\left|\begin{array}{cc}
x_{1}(n) & x_{2}(n) \\
x_{1}(n+1) & x_{2}(n+1)
\end{array}\right| \\
& =\left|\begin{array}{cc}
(q)^{\frac{n}{2}} \cdot \cos (n \cdot \varphi) & (q)^{\frac{n}{2}} \cdot \sin (n \cdot \varphi) \\
(q)^{\frac{n+1}{2}} \cdot \cos ((n+1) \cdot \varphi) & (q)^{\frac{n+1}{2}} \cdot \sin ((n+1) \cdot \varphi)
\end{array}\right| \\
& =(q)^{\frac{n}{2}} \cdot(q)^{\frac{n+1}{2}} \cdot(\sin ((n+1) \cdot \varphi) \cdot \cos (n \cdot \varphi) \\
& -\cos ((n+1) \cdot \varphi) \cdot \sin (n \cdot \varphi)) \\
& =(q)^{n} \cdot(q)^{\frac{1}{2}} \cdot \sin (\varphi) \neq 0,
\end{aligned}
$$

$\operatorname{denn} q \neq 0$ und $\sin (\varphi)=\frac{\beta}{\sqrt{\alpha^{2}+\beta^{2}}} \neq 0$.

\section{Folgerung 4.3.7}

Nach Satz 4.2.11 ist im Fall $\left(\frac{p}{2}\right)^{2}-q<0$ die Funktion $x: \mathbb{N}_{0} \rightarrow \mathbb{R}$ mit

$$
\begin{aligned}
x(n) & =c_{1} \cdot(q)^{\frac{n}{2}} \cdot \cos (n \cdot \varphi)+c_{2} \cdot(q)^{\frac{n}{2}} \cdot \sin (n \cdot \varphi) \\
& =(q)^{\frac{n}{2}} \cdot\left(c_{1} \cdot \cos (n \cdot \varphi)+c_{2} \cdot \sin (n \cdot \varphi)\right)
\end{aligned}
$$

und beliebigen $c_{1}, c_{2} \in \mathbb{R}$ die allgemeine Lösung von (4.3.1) auf $\mathbb{N}_{0}$.

Dabei ist $\varphi=\arccos \left(-\frac{p}{2 \sqrt{q}}\right)$.

\section{Beispiel 4.3.8}

i) $\quad x(n+2)+9 x(n)=0, \quad n \in \mathbb{N}_{0}$.

Die entsprechende charakteristische Gleichung lautet:

$$
\lambda^{2}+9=0 \quad \Leftrightarrow \quad \lambda= \pm \mathrm{i} \cdot 3 .
$$


Es gilt $\alpha=0, \beta=3$ und $\varphi=\frac{\pi}{2}$. Dann bilden die Funktionen $x_{1}, x_{2}$ : $\mathbb{N}_{0} \rightarrow \mathbb{R}$ mit $x_{1}(n):=3^{n} \cdot \cos \left(\frac{\pi}{2} \cdot n\right)$ und $x_{2}(n):=3^{n} \cdot \sin \left(\frac{\pi}{2} \cdot n\right)$ ein Fundamentalsystem der Gleichung $x(n+2)+9 x(n)=0$ auf $\mathbb{N}_{0}$.

Nach Folgerung 4.3.7 ist

$$
x(n)=3^{n} \cdot\left(c_{1} \cdot \cos \left(\frac{\pi}{2} \cdot n\right)+c_{2} \cdot \sin \left(\frac{\pi}{2} \cdot n\right)\right)
$$

mit beliebigen $c_{1}, c_{2} \in \mathbb{R}$ die allgemeine Lösung dieser Gleichung auf $\mathbb{N}_{0}$.

ii) $\quad x(n+2)+2 x(n+1)+5 x(n)=0, \quad n \in \mathbb{N}_{0}$.

Die entsprechende charakteristische Gleichung ist

$$
\lambda^{2}+2 \lambda+5=0 \quad \Leftrightarrow \quad \lambda=-1 \pm \mathrm{i} \cdot 2 .
$$

Es gilt $\alpha=-1, \beta=2$ und $\varphi=\arccos \left(-\frac{1}{\sqrt{5}}\right)$. Dann bilden die Funktionen $x_{1}, x_{2}: \mathbb{N}_{0} \rightarrow \mathbb{R}$ mit $x_{1}(n):=(\sqrt{5})^{n} \cdot \cos (n \cdot \varphi)$ und $x_{2}(n):=(\sqrt{5})^{n} \cdot \sin (n \cdot \varphi)$ ein Fundamentalsystem der homogenen Gleichung $x(n+2)+2 x(n+1)+5 x(n)=0$ auf $\mathbb{N}_{0}$.

Nach Folgerung 4.3.7 ist

$$
x(n)=5^{\frac{n}{2}} \cdot\left(c_{1} \cdot \cos (n \cdot \varphi)+c_{2} \cdot \sin (n \cdot \varphi)\right)
$$

mit beliebigen $c_{1}, c_{2} \in \mathbb{R}$ die allgemeine Lösung dieser Gleichung auf $\mathbb{N}_{0}$. Dabei ist $\varphi=\arccos \left(-\frac{1}{\sqrt{5}}\right)$.

\section{Beispiel 4.3.9 (FibonaccI-Zahlen ${ }^{10}$ )}

Definition: Die unendliche Folge $\left\langle F_{n}\right\rangle_{n \in \mathbb{N}_{0}}$ der natürlichen Zahlen, die rekursiv durch

$$
F_{n}:=F_{n-1}+F_{n-2}
$$

für $n \geqslant 2$ mit den Anfangswerten $F_{0}=0$ und $F_{1}=1$ definiert ist, heißt FIBonACCI- Folge. Die darin enthaltenen Zahlen heißen FibonAcci-Zahlen. Wir können diese als folgendes Anfangswertproblem auffassen:

$$
\left\{\begin{aligned}
x(n+2)-x(n+1)-x(n) & =0, \quad n \in \mathbb{N}_{0}, \\
x(0) & =0, \\
x(1) & =1 .
\end{aligned}\right.
$$

\footnotetext{
${ }^{10}$ Leonardo da Pisa, auch Fibonacci genannt, ca. 1170-1240
} 
Die charakteristische Gleichung der homogenen Differenzengleichung lautet

$$
\lambda^{2}-\lambda-1=0 \quad \Leftrightarrow \quad \lambda=\frac{1 \pm \sqrt{5}}{2} .
$$

Nach Folgerung 4.3.3 ist mit beliebigen $c_{1}, c_{2} \in \mathbb{R}$ die Funktion

$$
x(n)=c_{1} \cdot\left(\frac{1+\sqrt{5}}{2}\right)^{n}+c_{2} \cdot\left(\frac{1-\sqrt{5}}{2}\right)^{n}
$$

die allgemeine Lösung der homogenen Differenzengleichung. Wir suchen spezielle $c_{1}, c_{2} \in \mathbb{R}$, mit:

$$
\begin{aligned}
&\left\{\begin{array} { l } 
{ x ( 0 ) = 0 } \\
{ x ( 1 ) = 1 }
\end{array} \quad \Leftrightarrow \quad \left\{\begin{array}{l}
c_{1}+c_{2}=0 \\
c_{1} \cdot \frac{1+\sqrt{5}}{2}+c_{2} \cdot \frac{1-\sqrt{5}}{2}=1
\end{array}\right.\right. \\
& \Leftrightarrow\left\{\begin{array}{l}
c_{1}=\frac{1}{\sqrt{5}} \\
c_{2}=-\frac{1}{\sqrt{5}}
\end{array}\right.
\end{aligned}
$$

Dann gilt für jedes $n \in \mathbb{N}_{0}$

$$
x(n)=\frac{1}{\sqrt{5}} \cdot\left(\left(\frac{1+\sqrt{5}}{2}\right)^{n}-\left(\frac{1-\sqrt{5}}{2}\right)^{n}\right),
$$

welche Formel von BINET ${ }^{11}$ genannt wird.

\section{Übung}

Weisen Sie nach: Der Quotient zweier aufeinanderfolgender FibonacciZahlen nähert sich dem Goldenen Schnitt. Also gilt $\lim _{n \rightarrow \infty} \frac{F_{n+1}}{F_{n}}=\frac{1+\sqrt{5}}{2}$.

\section{Beispiel 4.3.10}

Für $a, b \in \mathbb{R} \backslash\{0\}$ und $\mu_{0}, \mu_{1} \in \mathbb{R}$ betrachten wir

$$
\left\{\begin{aligned}
x(n+2)-(a+b) \cdot x(n+1)+a \cdot b \cdot x(n) & =0, \quad n \in \mathbb{N}_{0}, \\
x(0) & =\mu_{0}, \\
x(1) & =\mu_{1} .
\end{aligned}\right.
$$

\footnotetext{
${ }^{11}$ Jacques Philippe Marie Binet, 1786-1856
} 
Die charakteristische Gleichung der homogenen Differenzengleichung lautet

$$
\lambda^{2}-(a+b) \cdot \lambda+a \cdot b=0 \quad \Leftrightarrow \quad \lambda=\frac{a+b}{2} \pm \frac{a-b}{2} .
$$

I. Fall $a \neq b$ Dann besitzt die charakteristische Gleichung zwei verschiedene reelle Lösungen. Seien $\lambda_{1}:=a, \lambda_{2}:=b$. Nach Folgerung 4.3.3 ist

$$
x(n)=c_{1} \cdot a^{n}+c_{2} \cdot b^{n}
$$

mit beliebigen $c_{1}, c_{2} \in \mathbb{R}$ die allgemeine Lösung der homogenen Differenzengleichung auf $\mathbb{N}_{0}$. Wir suchen spezielle $c_{1}, c_{2} \in \mathbb{R}$, mit:

$$
\left\{\begin{array} { l } 
{ x ( 0 ) = \mu _ { 0 } } \\
{ x ( 1 ) = \mu _ { 1 } }
\end{array} \Leftrightarrow \left\{\begin{array}{l}
c_{1}+c_{2}=\mu_{0} \\
c_{1} \cdot a+c_{2} \cdot b=\mu_{1}
\end{array}\right.\right.
$$

Es gilt:

$$
\triangle:=\left|\begin{array}{ll}
1 & 1 \\
a & b
\end{array}\right|=b-a \neq 0, \quad \text { denn } \quad a \neq b
$$

sowie

$$
\triangle_{1}:=\left|\begin{array}{ll}
\mu_{0} & 1 \\
\mu_{1} & b
\end{array}\right|=b \cdot \mu_{0}-\mu_{1} \quad \text { und } \quad \triangle_{2}:=\left|\begin{array}{cc}
1 & \mu_{0} \\
a & \mu_{1}
\end{array}\right|=\mu_{1}-a \cdot \mu_{0}
$$

Nach der CRAMER'schen Regel folgt:

$$
c_{1}=\frac{\triangle_{1}}{\triangle}=\frac{b \cdot \mu_{0}-\mu_{1}}{b-a}, \quad c_{2}=\frac{\triangle_{2}}{\triangle}=\frac{\mu_{1}-a \cdot \mu_{0}}{b-a} .
$$

Dann ist

$$
x(n)=\frac{\left(b \cdot \mu_{0}-\mu_{1}\right) \cdot a^{n}+\left(\mu_{1}-a \cdot \mu_{0}\right) \cdot b^{n}}{b-a}
$$

die eindeutige Lösung des Anfangswertproblems 4.3.10 im Fall $a \neq b$.

II. Fall $a=b$ Dann besitzt die charakteristische Gleichung eine reelle Lösung $\lambda=a$ (mit Vielfachheit $r=2$ ). Nach Folgerung 4.3.5 ist

$$
x(n)=a^{n} \cdot\left(c_{1}+c_{2} \cdot n\right)
$$

mit beliebigen $c_{1}, c_{2} \in \mathbb{R}$ die allgemeine Lösung der homogenen Differenzengleichung auf $\mathbb{N}_{0}$. 
Wir suchen spezielle $c_{1}, c_{2} \in \mathbb{R}$, mit:

$$
\begin{aligned}
\left\{\begin{array}{l}
x(0)=\mu_{0} \\
x(1)=\mu_{1}
\end{array}\right. & \Leftrightarrow\left\{\begin{array}{l}
c_{1}=\mu_{0} \\
a \cdot\left(c_{1}+c_{2}\right)=\mu_{1}
\end{array}\right. \\
& \Leftrightarrow \quad\left\{\begin{array}{l}
c_{1}=\mu_{0} \\
c_{2}=\frac{\mu_{1}-a \cdot \mu_{0}}{a}
\end{array}\right.
\end{aligned}
$$

Dann ist

$$
x(n)=\mu_{0} \cdot a^{n}+\left(\mu_{1}-a \cdot \mu_{0}\right) \cdot n \cdot a^{n-1}
$$

die eindeutige Lösung des Anfangswertproblems 4.3.10 im Fall $a=b$.

\section{Übung}

Weisen Sie nach: Fall I verhält sich wie Fall II, wenn $b \rightarrow a$ läuft.

\subsection{Lineare inhomogene Differenzengleichung zweiter Ordnung}

Satz 4.4.1

Gegeben seien die Funktionen $p, q, f: \mathbb{N}_{0} \rightarrow \mathbb{R}$, wobei $q(n) \neq 0$ für alle $n \in \mathbb{N}_{0}$ ist. Dann lässt sich die Lösungsmenge $\mathbb{L}$ der inhomogenen Differenzengleichung 2. Ordnung

$$
x(n+2)+p(n) \cdot x(n+1)+q(n) \cdot x(n)=f(n)
$$

auf $\mathbb{N}_{0}$ schreiben als: $\mathbb{L}=x^{*}+\mathbb{L}_{0}$, wobei $\mathbb{L}_{0}$ die Lösungsmenge der entsprechenden homogenen Gleichung

$$
x(n+2)+p(n) \cdot x(n+1)+q(n) \cdot x(n)=0
$$

und $x^{*}(n)$ eine (sog. partikuläre) Lösung der inhomogenen Gleichung ist. 


\section{BEWEIS:}

Wir haben bereits in Satz 4.2.15 gesehen, dass $\mathbb{L}_{0}$ nichtleer ist. Wir werden in Bemerkung 4.4.4 zeigen, dass auch $\mathbb{L} \neq \varnothing$, mit anderen Worten existiert eine partikuläre Lösung $x^{*}(n)$ von (4.4.1) und es gilt auf $\mathbb{N}_{0}$

$$
x^{*}(n+2)+p(n) \cdot x^{*}(n+1)+q(n) \cdot x^{*}(n)=f(n) .
$$

1. Sei $x_{L}(n) \in \mathbb{L}$ eine beliebige Lösung von (4.4.1). Dann gilt für jedes $n \in \mathbb{N}_{0}$

$$
x_{L}(n+2)+p(n) \cdot x_{L}(n+1)+q(n) \cdot x_{L}(n)=f(n),
$$

und somit

$$
\begin{aligned}
\left(x_{L}(n+2)-x^{*}(n+2)\right)+p(n) \cdot( & \left.x_{L}(n+1)-x^{*}(n+1)\right) \\
& +q(n) \cdot\left(x_{L}(n)-x^{*}(n)\right)=0 .
\end{aligned}
$$

D.h., $\widetilde{x}(n):=x_{L}(n)-x^{*}(n)$ ist eine Lösung der homogenen Gleichung

$$
x(n+2)+p(n) \cdot x(n+1)+q(n) \cdot x(n)=0
$$

auf $\mathbb{N}_{0}$. Also gilt für jedes $x_{L}(n) \in \mathbb{L}$ :

$$
x_{L}(n)=x^{*}(n)+\widetilde{x}(n) \in x^{*}(n)+\mathbb{L}_{0} \quad \text { und } \quad \mathbb{L} \subseteq x^{*}(n)+\mathbb{L}_{0} .
$$

2. Sei nun $\widetilde{x}(n) \in \mathbb{L}_{0}$ eine beliebige Lösung der homogenen Gleichung. Dann gilt für jedes $n \in \mathbb{N}_{0}$ :

$$
\widetilde{x}(n+2)+p(n) \cdot \widetilde{x}(n+1)+q(n) \cdot \widetilde{x}(n)=0
$$

Da $x^{*}(n)$ eine (partikuläre) Lösung der inhomogenen Gleichung (4.4.1) ist, folgt

$$
\begin{aligned}
\left(\widetilde{x}(n+2)+x^{*}(n+2)\right)+p(n) \cdot(\widetilde{x}(n+1) & \left.+x^{*}(n+1)\right) \\
& +q(n) \cdot\left(\widetilde{x}(n)+x^{*}(n)\right)=f(n)
\end{aligned}
$$

D. h., $x(n):=\widetilde{x}(n)+x^{*}(n)$ ist eine Lösung der inhomogenen Gleichung (4.4.1). Also gilt für jedes $\widetilde{x}(n) \in \mathbb{L}_{0}$

$$
x^{*}(n)+\widetilde{x}(n) \in \mathbb{L} \quad \text { und } \quad x^{*}(n)+\mathbb{L}_{0} \subseteq \mathbb{L} .
$$

Insgesamt haben wir $\mathbb{L}=x^{*}(n)+\mathbb{L}_{0}$ gezeigt. 


\section{Folgerung 4.4.2}

Sei $\left\{x_{1}, x_{2}\right\}$ ein Fundamentalsystem der homogenen Differenzengleichung

$$
x(n+2)+p(n) \cdot x(n+1)+q(n) \cdot x(n)=0
$$

auf $\mathbb{N}_{0}$. Lässt man in $c_{1} \cdot x_{1}(n)+c_{2} \cdot x_{2}(n)$ die Koeffizienten $c_{1}, c_{2} \in \mathbb{R}$ unabhängig voneinander die Menge der reellen Zahlen durchlaufen, so erhält man alle Lösungen der homogenen Gleichung (4.4.1h).

Also gilt für die Lösungsmenge

$$
\mathbb{L}_{0}=\left\{c_{1} \cdot x_{1}(n)+c_{2} \cdot x_{2}(n): \quad c_{1}, c_{2} \in \mathbb{R}\right\},
$$

dabei heißt $c_{1} \cdot x_{1}(n)+c_{2} \cdot x_{2}(n)$ allgemeine Lösung der homogenen Gleichung. Ist jetzt $x^{*}(n)$ eine partikuläre Lösung der inhomogenen Gleichung (4.4.1), dann gilt nach Satz 4.4.1 für die Lösungsmenge:

$$
\mathbb{L}=\left\{c_{1} \cdot x_{1}(n)+c_{2} \cdot x_{2}(n)+x^{*}(n): c_{1}, c_{2} \in \mathbb{R}\right\} .
$$

Man sagt: Durch $x(n)=c_{1} \cdot x_{1}(n)+c_{2} \cdot x_{2}(n)+x^{*}(n)$ ist die allgemeine Lösung der linearen inhomogenen Differenzengleichung 2. Ordnung (4.4.1) gegeben.

Oder auch: Die allgemeine Lösung der linearen inhomogenen Differenzengleichung 2. Ordnung hat die Form

$$
x(n)=\widetilde{x}(n)+x^{*}(n),
$$

wobei $\widetilde{x}(n)$ die allgemeine Lösung der entsprechenden linearen homogenen Gleichung und $x^{*}(n)$ eine partikuläre Lösung der inhomogenen Gleichung ist.

\section{Beispiel 4.4.3}

$$
x(n+2)-9 x(n)=n, \quad n \in \mathbb{N}_{0} .
$$

1) Die entsprechende homogene Gleichung lautet

$$
x(n+2)-9 x(n)=0
$$

und die charakteristische Gleichung ist

$$
\lambda^{2}-9=0 \quad \Leftrightarrow \quad \lambda= \pm 3 .
$$


Nach Folgerung 4.3.3 ist

$$
\widetilde{x}(n):=c_{1} \cdot 3^{n}+c_{2} \cdot(-3)^{n} \quad \text { mit beliebigen } \quad c_{1}, c_{2} \in \mathbb{R}
$$

die allgemeine Lösung der entsprechenden homogenen Gleichung (4.4.3h).

2) Wie ist nun $x^{*}(n)$ zu wählen?

Dazu betrachten wir den Ansatz $x^{*}(n):=A \cdot n+B$ mit $A, B \in \mathbb{R}$. Dann gilt für jedes $n \in \mathbb{N}_{0}$ :

$$
x^{*}(n+2)-9 x^{*}(n)=n, \quad \Leftrightarrow \quad A \cdot(n+2)+B-9 \cdot(A \cdot n+B)=n .
$$

Ein Koeffizientenvergleich liefert:

$$
\left\{\begin{array} { l } 
{ - 8 A = 1 } \\
{ 2 A - 8 B = 0 }
\end{array} \quad \Leftrightarrow \quad \left\{\begin{array}{l}
A=-\frac{1}{8} \\
B=-\frac{1}{32}
\end{array}\right.\right.
$$

Dann ist $x^{*}(n):=-\frac{1}{8} \cdot n-\frac{1}{32}$ eine partikuläre Lösung der inhomogenen Gleichung (4.4.3). Nach Satz 4.4.1 und Folgerung 4.4.2 ist

$$
x(n)=\widetilde{x}(n)+x^{*}(n)=c_{1} \cdot 3^{n}+c_{2} \cdot(-3)^{n}+\left(-\frac{1}{8} \cdot n-\frac{1}{32}\right)
$$

die allgemeine Lösung der inhomogenen Differenzengleichung (4.4.3) auf $\mathbb{N}_{0}$.

\section{Bemerkung 4.4.4 (Variation der Konstanten)}

Gegeben seien die Funktionen $p, q, f: \mathbb{N}_{0} \rightarrow \mathbb{R}$, wobei $q(n) \neq 0$ für alle $n \in \mathbb{N}_{0}$ ist. Sei weiter $\left\{x_{1}, x_{2}\right\}$ ein Fundamentalsystem der homogenen Gleichung

$$
x(n+2)+p(n) \cdot x(n+1)+q(n) \cdot x(n)=0 .
$$

Eine partikuläre Lösung der inhomogenen Gleichung

$$
x(n+2)+p(n) \cdot x(n+1)+q(n) \cdot x(n)=f(n)
$$

findet man nach dem Verfahren der Variation der Konstanten: 
Wir suchen eine partikuläre Lösung in der Form:

$$
x^{*}(n)=c_{1}(n) \cdot x_{1}(n)+c_{2}(n) \cdot x_{2}(n)
$$

wobei $c_{1}, c_{2}: \mathbb{N}_{0} \rightarrow \mathbb{R}$ unbekannte Funktionen sind. Dann gilt (nach Einsetzung in (4.4.1)):

$$
\begin{aligned}
& c_{1}(n+2) \cdot x_{1}(n+2)+c_{2}(n+2) \cdot x_{2}(n+2) \\
& \quad+p(n) \cdot\left(c_{1}(n+1) \cdot x_{1}(n+1)+c_{2}(n+1) \cdot x_{2}(n+1)\right) \\
& \quad+q(n) \cdot\left(c_{1}(n) \cdot x_{1}(n)+c_{2}(n) \cdot x_{2}(n)\right)=f(n) .
\end{aligned}
$$

Da die Funktionen $x_{1}, x_{2}$ die Lösungen der homogenen Gleichung sind gilt:

$$
\begin{aligned}
& c_{1}(n) \cdot x_{1}(n+2)+c_{1}(n) \cdot p(n) \cdot x_{1}(n+1) \\
& +c_{1}(n) \cdot q(n) \cdot x_{1}(n)=0, \\
& c_{2}(n) \cdot x_{2}(n+2)+c_{2}(n) \cdot p(n) \cdot x_{2}(n+1) \\
& +c_{2}(n) \cdot q(n) \cdot x_{2}(n)=0 .
\end{aligned}
$$

Dann liefert $(*)-(* *)-(* * *)$ :

$$
\begin{gathered}
\left(c_{1}(n+2)-c_{1}(n)\right) \cdot x_{1}(n+2) \\
+\left(c_{2}(n+2)-c_{2}(n)\right) \cdot x_{2}(n+2) \\
+p(n) \cdot\left(\left(c_{1}(n+1)-c_{1}(n)\right) \cdot x_{1}(n+1)\right. \\
\left.+\left(c_{2}(n+1)-c_{2}(n)\right) \cdot x_{2}(n+1)\right)=f(n) \\
\Leftrightarrow \quad\left(\left(c_{1}(n+2)-c_{1}(n+1)\right)+\left(c_{1}(n+1)-c_{1}(n)\right)\right) \cdot x_{1}(n+2) \\
+\left(\left(c_{2}(n+2)-c_{2}(n+1)\right)+\left(c_{2}(n+1)-c_{2}(n)\right)\right) \cdot x_{2}(n+2) \\
+p(n) \cdot\left(\left(c_{1}(n+1)-c_{1}(n)\right) \cdot x_{1}(n+1)\right. \\
\left.+\left(c_{2}(n+1)-c_{2}(n)\right) \cdot x_{2}(n+1)\right)=f(n)
\end{gathered}
$$




$$
\begin{aligned}
& \Leftrightarrow \quad\left(c_{1}(n+2)-c_{1}(n+1)\right) \cdot x_{1}(n+2) \\
& +\left(c_{2}(n+2)-c_{2}(n+1)\right) \cdot x_{2}(n+2) \\
& +\left(c_{1}(n+1)-c_{1}(n)\right) \cdot x_{1}(n+2) \\
& \quad+\left(c_{2}(n+1)-c_{2}(n)\right) \cdot x_{2}(n+2) \\
& +p(n) \cdot\left(\left(c_{1}(n+1)-c_{1}(n)\right) \cdot x_{1}(n+1)\right. \\
& \left.+\left(c_{2}(n+1)-c_{2}(n)\right) \cdot x_{2}(n+1)\right)=f(n) . \\
& \quad(* * * *)
\end{aligned}
$$

Als zusätzliche Bedingung auf $\mathbb{N}_{0}$ nehmen wir an:

$$
\begin{aligned}
\left(c_{1}(n+1)-\right. & \left.c_{1}(n)\right) \cdot x_{1}(n+1) \\
& +\left(c_{2}(n+1)-c_{2}(n)\right) \cdot x_{2}(n+1)=0 .
\end{aligned}
$$

Dann ist

$$
\begin{aligned}
\left(c_{1}(n+2)-\right. & \left.c_{1}(n+1)\right) \cdot x_{1}(n+2) \\
& +\left(c_{2}(n+2)-c_{2}(n+1)\right) \cdot x_{2}(n+2)=0 .
\end{aligned}
$$

Und eingesetzt in $(* * * *)$ :

$$
\begin{aligned}
\left(c_{1}(n+1)-\right. & \left.c_{1}(n)\right) \cdot x_{1}(n+2) \\
& +\left(c_{2}(n+1)-c_{2}(n)\right) \cdot x_{2}(n+2)=f(n) .
\end{aligned}
$$

Wir setzten

$$
w_{1}(n):=c_{1}(n+1)-c_{1}(n) \quad \text { und } \quad w_{2}(n):=c_{2}(n+1)-c_{2}(n) .
$$

Mit Hilfe von $(+)$ und $(++)$ erhalten wir ein lineares Gleichungssystem für die unbekannten Funktionen $w_{1}(n), w_{2}(n): \mathbb{N}_{0} \rightarrow \mathbb{R}$, nämlich:

$$
\left\{\begin{array}{l}
w_{1}(n) \cdot x_{1}(n+1)+w_{2}(n) \cdot x_{2}(n+1)=0 \\
w_{1}(n) \cdot x_{1}(n+2)+w_{2}(n) \cdot x_{2}(n+2)=f(n)
\end{array}\right.
$$

mit der Hauptdeterminante

$$
\triangle:=\left|\begin{array}{ll}
x_{1}(n+1) & x_{2}(n+1) \\
x_{1}(n+2) & x_{2}(n+2)
\end{array}\right| .
$$


Nach Definition 4.2.7 ist $\Delta=D(n+1)$ die CASORATI-Determinante der Funktionen $x_{1}, x_{2}$. Da $\left\{x_{1}, x_{2}\right\}$ ein Fundamentalsystem der homogenen Gleichung ist, gilt $D(n+1) \neq 0$ für alle $n \in \mathbb{N}_{0}$. Dann ist das lineare Gleichungssystem mit den Unbekannten $w_{1}(n), w_{2}(n)$ eindeutig lösbar. Mit der CRAMER'schen Regel erhalten wir

$$
\triangle_{1}:=\left|\begin{array}{cc}
0 & x_{2}(n+1) \\
f(n) & x_{2}(n+2)
\end{array}\right|, \quad \triangle_{2}=\left|\begin{array}{cc}
x_{1}(n+1) & 0 \\
x_{1}(n+2) & f(n)
\end{array}\right|
$$

und

$$
\begin{aligned}
& w_{1}(n)=\frac{\triangle_{1}}{\triangle}=-\frac{x_{2}(n+1) \cdot f(n)}{D(n+1)}, \\
& w_{2}(n)=\frac{\triangle_{2}}{\triangle}=\frac{x_{1}(n+1) \cdot f(n)}{D(n+1)} .
\end{aligned}
$$

Da wir

$$
w_{1}(n):=c_{1}(n+1)-c_{1}(n)
$$

und

$$
w_{2}(n):=c_{2}(n+1)-c_{2}(n) .
$$

gesetzt haben, folgt

$$
c_{1}(n+1)=\sum_{k=0}^{n} w_{1}(k)+c_{1}(0) \quad b z w . \quad c_{2}(n+1)=\sum_{k=0}^{n} w_{2}(k)+c_{2}(0),
$$

wobei $c_{1}(0), c_{2}(0) \in \mathbb{R}$ beliebig gewählt werden können. Also gilt für jedes $n \in \mathbb{N}_{0}$ :

$$
c_{1}(n)=\sum_{k=0}^{n-1} w_{1}(k)+c_{1}(0) \quad b z w . \quad c_{2}(n)=\sum_{k=0}^{n-1} w_{2}(k)+c_{2}(0) .
$$

Wir erhalten die Funktionen $c_{1}, c_{2}: \mathbb{N}_{0} \rightarrow \mathbb{R}$ und somit eine partikuläre Lösung der inhomogenen Differenzengleichung in der Form

$$
x^{*}(n)=c_{1}(n) \cdot x_{1}(n)+c_{2}(n) \cdot x_{2}(n) .
$$




\section{Beispiel 4.4.5}

$$
x(n+2)-x(n)=\frac{2}{n^{2}+4 n+3}, \quad n \in \mathbb{N}_{0} .
$$

1) Die entsprechende homogene Gleichung lautet

$$
x(n+2)-x(n)=0
$$

und die charakteristische Gleichung ist

$$
\lambda^{2}-1=0 \quad \Leftrightarrow \quad \lambda= \pm 1
$$

Seien $\lambda_{1}:=1$ und $\lambda_{2}:=-1$. Nach Folgerung 4.3 .3 ist

$$
\widetilde{x}(n)=c_{1}+c_{2} \cdot(-1)^{n}
$$

die allgemeine Lösung der entsprechenden homogenen Gleichung (4.4.5h). Die Funktionen $x_{1}(n):=1$ und $x_{2}(n):=(-1)^{n}$ bilden ein Fundamentalsystem der homogenen Gleichung auf $\mathbb{N}_{0}$.

2) Wir suchen nun $x^{*}$ mit dem Verfahren der Variation der Konstanten. Nach Bemerkung 4.4.4 erhalten wir für alle $n \in \mathbb{N}_{0}$ :

$$
\left\{\begin{array}{l}
w_{1}(n) \cdot 1+w_{2}(n) \cdot(-1)^{n+1}=0 \\
w_{1}(n) \cdot 1+w_{2}(n) \cdot(-1)^{n+2}=\frac{2}{n^{2}+4 n+3}
\end{array}\right.
$$

mit der Hauptdeterminante

$$
\begin{aligned}
\Delta & :=D(n+1)=\left|\begin{array}{ll}
x_{1}(n+1) & x_{2}(n+1) \\
x_{1}(n+2) & x_{2}(n+2)
\end{array}\right|=\left|\begin{array}{cc}
1 & (-1)^{n+1} \\
1 & (-1)^{n+2}
\end{array}\right| \\
& =(-1)^{n+2}-(-1)^{n+1}=2 \cdot(-1)^{n}
\end{aligned}
$$

sowie

$$
\Delta_{1}:=\left|\begin{array}{cc}
0 & (-1)^{n+1} \\
\frac{2}{n^{2}+4 n+3} & (-1)^{n+2}
\end{array}\right|=\frac{-2 \cdot(-1)^{n+1}}{n^{2}+4 n+3}=\frac{2 \cdot(-1)^{n}}{n^{2}+4 n+3},
$$


und

$$
\Delta_{2}:=\left|\begin{array}{cc}
1 & 0 \\
1 & \frac{2}{n^{2}+4 n+3}
\end{array}\right|=\frac{2}{n^{2}+4 n+3} .
$$

Nach der Cramer'schen Regel gilt für jedes $n \in \mathbb{N}_{0}$ :

$$
w_{1}(n)=\frac{\triangle_{1}}{\triangle}=\frac{1}{n^{2}+4 n+3}=\frac{1}{2} \cdot\left[\frac{1}{n+1}-\frac{1}{n+3}\right]
$$

und

$$
\begin{aligned}
w_{2}(n) & =\frac{\triangle_{2}}{\triangle}=\frac{1}{(-1)^{n} \cdot\left(n^{2}+4 n+3\right)}=\frac{(-1)^{n}}{n^{2}+4 n+3} \\
& =\frac{(-1)^{n}}{2} \cdot\left[\frac{1}{n+1}-\frac{1}{n+3}\right] .
\end{aligned}
$$

Weiter gilt nach Bemerkung 4.4.4:

$$
\begin{aligned}
c_{1}(n) & =\sum_{k=0}^{n-1} w_{1}(k)+c_{1}(0)=\sum_{k=0}^{n-1} \frac{1}{2} \cdot\left(\frac{1}{k+1}-\frac{1}{k+3}\right)+c_{1}(0) \\
& =\frac{1}{2} \cdot\left[\left(1+\frac{1}{2}\right)-\left(\frac{1}{n+1}+\frac{1}{n+2}\right)\right]+c_{1}(0)
\end{aligned}
$$

und

$$
\begin{aligned}
c_{2}(n) & =\sum_{k=0}^{n-1} w_{2}(k)+c_{2}(0)=\sum_{k=0}^{n-1} \frac{(-1)^{k}}{2} \cdot\left(\frac{1}{k+1}-\frac{1}{k+3}\right)+c_{2}(0) \\
& =\frac{1}{2} \cdot\left[\left(1-\frac{1}{2}\right)-(-1)^{n} \cdot\left(\frac{1}{n+1}-\frac{1}{n+2}\right)\right]+c_{2}(0) .
\end{aligned}
$$

Mit $c_{1}(0)=-\frac{3}{4}$ und $c_{2}(0)=-\frac{1}{4}$ bekommen wir folgende partikuläre Lösung der inhomogenen Differenzengleichung (4.4.5):

$$
\begin{aligned}
x^{*}(n) & =c_{1}(n) \cdot x_{1}(n)+c_{2}(n) \cdot x_{2}(n) \\
& =-\frac{1}{2} \cdot\left[\frac{1}{n+1}+\frac{1}{n+2}\right]-\frac{(-1)^{n}}{2} \cdot\left[\frac{1}{n+1}-\frac{1}{n+2}\right] \cdot(-1)^{n} \\
& =-\frac{1}{2} \cdot\left[\frac{1}{n+1}+\frac{1}{n+2}\right]-\frac{1}{2} \cdot\left[\frac{1}{n+1}-\frac{1}{n+2}\right]=-\frac{1}{n+1} .
\end{aligned}
$$

Die allgemeine Lösung der inhomogenen Gleichung (4.4.5) auf $\mathbb{N}_{0}$ ist somit

$$
x(n)=\widetilde{x}(n)+x^{*}(n)=c_{1}+c_{2} \cdot(-1)^{n}-\frac{1}{n+1}
$$

mit beliebigen $c_{1}, c_{2} \in \mathbb{R}$. 


\section{Satz 4.4.6 (Superpositionsprinzip)}

Gegeben seien die Funktionen $p, q, f_{1}, f_{2}: \mathbb{N}_{0} \rightarrow \mathbb{R}$, wobei $q(n) \neq 0$ für alle $n \in \mathbb{N}_{0}$ ist. Seien weiter $x_{1}^{*}(n)$ eine partikuläre Lösung von

$$
x(n+2)+p(n) \cdot x(n+1)+q(n) \cdot x(n)=f_{1}(n)
$$

und $x_{2}^{*}(n)$ eine partikuläre Lösung von

$$
x(n+2)+p(n) \cdot x(n+1)+q(n) \cdot x(n)=f_{2}(n) .
$$

Dann ist $x^{*}(n):=x_{1}^{*}(n)+x_{2}^{*}(n)$ eine partikuläre Lösung von

$$
x(n+2)+p(n) \cdot x(n+1)+q(n) \cdot x(n)=f_{1}(n)+f_{2}(n) .
$$

BeweIs:

Einsetzen von $x^{*}(n)$ in (4.4.7) liefert die Behauptung, weil $x_{1}^{*}(n)$ und $x_{2}^{*}(n)$ partikuläre Lösungen der entsprechenden Gleichungen sind.

\subsection{Lineare inhomogene Differenzengleichung zweiter Ordnung mit konstanten Koeffizien- ten}

\section{Definition 4.5.1}

Gegeben seien eine Funktion $f: \mathbb{N}_{0} \rightarrow \mathbb{R}$ und reelle Zahlen $p, q \in \mathbb{R}$ mit $q \neq 0$.

Gesucht ist eine Funktion $x: \mathbb{N}_{0} \rightarrow \mathbb{R}$, sodass für alle $n \in \mathbb{N}_{0}$ gilt:

$$
x(n+2)+p \cdot x(n+1)+q \cdot x(n)=f(n) .
$$

Die Gleichung (4.5.1) heißt lineare inhomogene Differenzengleichung zweiter Ordnung mit konstanten Koeffizienten. Die Funktion $x: \mathbb{N}_{0} \rightarrow \mathbb{R}$ heißt Lösung der Gleichung (4.5.1) auf $\mathbb{N}_{0}$. (Vgl. Definition 4.1.1, Bemerkung 4.1.3, Definition 4.3.1). 


\section{Bemerkung 4.5.2}

Nach Folgerung 4.4.2 hat die allgemeine Lösung der linearen inhomogenen Differenzengleichung (4.5.1) die Form

$$
x(n)=\widetilde{x}(n)+x^{*}(n),
$$

wobei $\widetilde{x}(n)$ die allgemeine Lösung der entsprechenden linearen homogenen Differenzengleichung

$$
x(n+2)+p \cdot x(n+1)+q \cdot x(n)=0
$$

und $x^{*}(n)$ eine partikuläre Lösung der linearen inhomogenen Differenzengleichung (4.5.1) ist. Die allgemeine Lösung $\widetilde{x}(n)$ von $(4.5 .1 \mathrm{~h})$ bekommt man, wenn man die charakteristische Gleichung

$$
\lambda^{2}+p \cdot \lambda+q=0
$$

untersucht (s. Folgerungen 4.3.3, 4.3.5, 4.3.7).

Wir betrachten nun die Form partikulärer Lösungen $x^{*}(n)$ von (4.5.1) für einige Sonderfälle der rechten Seite $f: \mathbb{N}_{0} \rightarrow \mathbb{R}$ :

i) $f(n):=P_{m}(n)$ ein Polynom vom Grad $m \in \mathbb{N}_{0}$.

a) $\lambda=1$ ist keine Lösung der charakteristischen Gleichung (4.5.1c).

Wir suchen eine partikuläre Lösung in der Form

$$
x^{*}(n)=Q_{m}(n),
$$

wobei $Q_{m}(n)$ ein Polynom vom Grad $m$ mit unbekannten Koeffizienten ist.

(s. Beispiel 4.5.4: $\left.\quad x(n+2)-x(n+1)+x(n)=n^{3}+6\right)$

b) $\lambda=1$ ist eine Lösung der charakteristischen Gleichung (4.5.1c) (mit Vielfachheit $r \in \mathbb{N}$ ).

Wir suchen eine partikuläre Lösung in der Form

$$
x^{*}(n)=n^{r} \cdot Q_{m}(n),
$$

wobei $Q_{m}(n)$ ein Polynom vom Grad $m$ mit unbekannten Koeffizienten ist.

(s. Beispiel 4.5.5: $\quad x(n+2)-2 x(n+1)+x(n)=2$ ) 
ii) $f(n):=\gamma^{n} \cdot P_{m}(n)$, wobei $\gamma \in \mathbb{R} \backslash\{0,1\}$ und $P_{m}(n)$ ein Polynom vom Grad $m \in \mathbb{N}_{0}$ ist.

a) $\lambda=\gamma$ ist keine Lösung der charakteristischen Gleichung (4.5.1c).

Wir suchen eine partikuläre Lösung in der Form

$$
x^{*}(n)=\gamma^{n} \cdot Q_{m}(n),
$$

wobei $Q_{m}(n)$ ein Polynom vom Gradm mit unbekannten Koeffizienten ist. (s. Beispiel 4.5.6: $\left.\quad x(n+2)-3 x(n+1)-4 x(n)=(6 n-8) \cdot 2^{n}\right)$

b) $\lambda=\gamma$ ist eine Lösung der charakteristischen Gleichung (4.5.1c) (mit Vielfachheit $r \in \mathbb{N}$ ).

Wir suchen eine partikuläre Lösung in der Form

$$
x^{*}(n)=n^{r} \cdot \gamma^{n} \cdot Q_{m}(n),
$$

wobei $Q_{m}(n)$ ein Polynom vom Grad $m$ mit unbekannten Koeffizienten ist. (s. Beispiel 4.5.7: $\left.\quad x(n+2)+5 x(n+1)+6 x(n)=(8 n-2) \cdot(-2)^{n}\right)$

iii) $f(n):=P_{m}(n) \cdot \cos (n \psi)+Q_{l}(n) \cdot \sin (n \psi)$, wobei $\psi \in \mathbb{R} \backslash\{\pi k\}$ mit $k \in \mathbb{Z}$. Dabei sind $P_{m}(n), Q_{l}(n)$ Polynome vom Grad $m, l \in \mathbb{N}_{0}$ oder genau ein Polynom ist das Nullpolynom.

a) $\lambda=\cos (\psi) \pm \mathrm{i} \cdot \sin (\psi)$ sind keine Lösungen der charakteristischen Gleichung (4.5.1c).

Wir suchen eine partikuläre Lösung in der Form

$$
x^{*}(n)=S_{N}(n) \cdot \cos (n \psi)+T_{N}(n) \cdot \sin (n \psi),
$$

wobei $S_{N}(n), T_{N}(n)$ Polynome vom Grad nicht mehr als $N:=\max \{m, l\}$ mit unbekannten Koeffizienten sind.

(s. Beispiel 4.5.8: $\left.\quad x(n+2)+4 x(n)=17 n \cdot \sin \left(\frac{\pi}{4} \cdot n\right)\right)$ 
b) $\lambda=\cos (\psi) \pm \mathrm{i} \cdot \sin (\psi)$ sind Lösungen der charakteristischen Gleichung (4.5.1c) (mit Vielfachheit $r \in \mathbb{N}$ ).

Wir suchen eine partikuläre Lösung in der Form

$$
x^{*}(n)=n^{r} \cdot\left(S_{N}(n) \cdot \cos (n \psi)+T_{N}(n) \cdot \sin (n \psi)\right),
$$

wobei $S_{N}(n), T_{N}(n)$ Polynome vom Grad nicht mehr als $N:=\max \{m, l\}$ mit unbekannten Koeffizienten sind.

(s. Beispiel 4.5.9: $\left.\quad x(n+2)+x(n)=\cos \left(\frac{\pi}{2} \cdot n\right)\right)$

iv) $f(n):=\rho^{n} \cdot\left(P_{m}(n) \cdot \cos (n \psi)+Q_{l}(n) \cdot \sin (n \psi)\right)$, wobei $\rho \in \mathbb{R}$ mit $\rho>0$ und $\rho \neq 1, \psi \in \mathbb{R} \backslash\{\pi k\}$ mit $k \in \mathbb{Z}$. Dabei sind $P_{m}(n), Q_{l}(n)$ Polynome vom Grad $m, l \in \mathbb{N}_{0}$ oder genau ein Polynom ist das Nullpolynom.

a) $\lambda=\rho \cdot(\cos (\psi) \pm \mathrm{i} \cdot \sin (\psi))$ sind keine Lösungen der charakteristischen Gleichung (4.5.1c).

Wir suchen eine partikuläre Lösung in der Form

$$
x^{*}(n)=\rho^{n} \cdot\left(S_{N}(n) \cdot \cos (n \psi)+T_{N}(n) \cdot \sin (n \psi)\right),
$$

wobei $S_{N}(n), T_{N}(n)$ Polynome vom Grad nicht mehr als $N:=\max \{m, l\}$ mit unbekannten Koeffizienten sind.

(s. Beispiel 4.5.10: $\left.\quad x(n+2)+2 x(n+1)+4 x(n)=2^{n} \cdot \sin \left(\frac{\pi}{2} \cdot n\right)\right)$

b) $\lambda=\rho \cdot(\cos (\psi) \pm \mathrm{i} \cdot \sin (\psi))$ sind Lösungen der charakteristischen Gleichung (4.5.1c) (mit Vielfachheit $r \in \mathbb{N}$ ).

Wir suchen eine partikuläre Lösung in der Form

$$
x^{*}(n)=n^{r} \cdot \rho^{n} \cdot\left(S_{N}(n) \cdot \cos (n \psi)+T_{N}(n) \cdot \sin (n \psi)\right),
$$

wobei $S_{N}(n), T_{N}(n)$ Polynome vom Grad nicht mehrals $N:=\max \{m, l\}$ mit unbekannten Koeffizienten sind.

(s. Beispiel 4.5.11: $\left.x(n+2)+9 x(n)=3^{n+2} \cdot \cos \left(\frac{\pi}{2} \cdot n\right)\right)$

\section{Bemerkung 4.5.3 ( zu den Teilen iii und iv)}

Für alle $\psi \in \mathbb{R}$ und $n \in \mathbb{Z}$ gilt:

$$
\cos (n \cdot(\psi+\pi))=\cos (n \psi) \cdot(-1)^{n}, \quad \sin (n \cdot(\psi+\pi))=\sin (n \psi) \cdot(-1)^{n}
$$


Sei $f: \mathbb{N}_{0} \rightarrow \mathbb{R}$ eine Funktion mit

$$
f(n):=(-1)^{n} \cdot\left[P_{m}(n) \cdot \cos (n \psi)+Q_{l}(n) \cdot \sin (n \psi)\right]
$$

$b z w$.

$$
f(n):=(-1)^{n} \cdot \rho^{n} \cdot\left[P_{m}(n) \cdot \cos (n \psi)+Q_{l}(n) \cdot \sin (n \psi)\right],
$$

wobei $\rho \in \mathbb{R}$ mit $\rho>0$ und $\rho \neq 1, \psi \in \mathbb{R} \backslash\{\pi k\}$ mit $k \in \mathbb{Z}$. Ferner sind $P_{m}(n), Q_{l}(n)$ Polynome vom Grad $m, l \in \mathbb{N}_{0}$ oder genau ein Polynom ist das Nullpolynom. Dann gilt

$$
f(n)=P_{m}(n) \cdot \cos (n(\psi+\pi))+Q_{l}(n) \cdot \sin (n(\psi+\pi))
$$

$b z w$.

$$
f(n)=\rho^{n} \cdot\left[P_{m}(n) \cdot \cos (n(\psi+\pi))+Q_{l}(n) \cdot \sin (n(\psi+\pi))\right] .
$$

Wir setzen nun $\theta:=\psi+\pi$ und suchen eine partikuläre Lösung der inhomogenen Differenzengleichung (4.4.1) wie in iii) bzw. iv). Wie oben, ist es entscheidend, ob $\lambda=\cos (\theta) \pm \mathrm{i} \cdot \sin (\theta) b z w \cdot \lambda=\rho \cdot(\cos (\theta) \pm \mathrm{i} \cdot \sin (\theta))$ keine Lösungen oder sehr wohl Lösungen (jeweils mit Vielfachheit $r$ ) der charakteristischen Gleichung (4.3.1c) sind.

(s. Beispiel 4.5.12: $\left.\quad x(n+2)+\sqrt{3} \cdot x(n+1)+x(n)=(-1)^{n} \cdot \sin \left(\frac{\pi}{6} \cdot n\right)\right)$

\section{Beispiel 4.5.4}

$$
x(n+2)-x(n+1)+x(n)=n^{3}+6, \quad n \in \mathbb{N}_{0} .
$$

1) $x(n+2)-x(n+1)+x(n)=0$ ist die entsprechende homogene Gleichung. Die charakteristische Gleichung lautet

$$
\left.\lambda^{2}-\lambda+1=0 \Leftrightarrow \lambda=\frac{1}{2} \pm \mathrm{i} \cdot \frac{\sqrt{3}}{2} \text { (jeweils mit Vielfachheit } r=1\right) .
$$

Hier gilt $\alpha=\frac{1}{2}, \beta=\frac{\sqrt{3}}{2}, \varphi=\arccos \left(\frac{1}{2}\right)=\frac{\pi}{3}, q=1$, und nach Folgerung 4.3.7 ist

$$
\widetilde{x}(n)=c_{1} \cdot \cos \left(\frac{\pi}{3} \cdot n\right)+c_{2} \cdot \sin \left(\frac{\pi}{3} \cdot n\right)
$$

mit beliebigen $c_{1}, c_{2} \in \mathbb{R}$ die allgemeine Lösung der entsprechenden homogenen Gleichung auf $\mathbb{N}_{0}$. 
2) Wir haben $P_{3}(n)=n^{3}+6$. Da 1 keine Lösung unserer charakteristischen Gleichung ist, suchen wir nach Bemerkung 4.5.2 eine partikuläre Lösung der inhomogenen Gleichung in der Form

$$
x^{*}(n)=A \cdot n^{3}+B \cdot n^{2}+C \cdot n+D \quad \text { mit } A, B, C, D \in \mathbb{R} .
$$

Einsetzen in die inhomogene Gleichung (4.5.4) ergibt:

$$
\begin{aligned}
&\left(A \cdot(n+2)^{3}+B \cdot(n+2)^{2}+C \cdot(n+2)+D\right) \\
&-\left(A \cdot(n+1)^{3}+B \cdot(n+1)^{2}+C \cdot(n+1)+D\right) \\
&+C \cdot(n+1)+D)+\left(A \cdot n^{3}+B \cdot n^{2}+C \cdot n+D\right)=n^{3}+6 \\
& \Leftrightarrow \quad A \cdot n^{3}+(3 A+B) \cdot n^{2}+(9 A+2 B+C) \cdot n \\
&+(7 A+3 B+C+D)=n^{3}+6 .
\end{aligned}
$$

Ein Koeffizientenvergleich liefert:

$$
\left\{\begin{array} { l } 
{ A = 1 } \\
{ 3 A + B = 0 } \\
{ 9 A + 2 B + C = 0 } \\
{ 7 A + 3 B + C + D = 6 }
\end{array} \Leftrightarrow \left\{\begin{array}{l}
A=1 \\
B=-3 \\
C=-3 \\
D=11
\end{array}\right.\right.
$$

Dann ist

$$
x^{*}(n)=n^{3}-3 n^{2}-3 n+11
$$

eine partikuläre Lösung und

$$
\begin{aligned}
x(n) & =\widetilde{x}(n)+x^{*}(n) \\
& =c_{1} \cdot \cos \left(\frac{\pi}{3} \cdot n\right)+c_{2} \cdot \sin \left(\frac{\pi}{3} \cdot n\right)+n^{3}-3 n^{2}-3 n+11
\end{aligned}
$$

die allgemeine Lösung der inhomogenen Gleichung (4.5.4) auf $\mathbb{N}_{0}$. 


\section{Beispiel 4.5.5}

$$
x(n+2)-2 x(n+1)+x(n)=2, \quad n \in \mathbb{N}_{0} .
$$

1) $x(n+2)-2 x(n+1)+x(n)=0$ ist die entsprechende homogene Gleichung. Die charakteristische Gleichung lautet

$$
\lambda^{2}-2 \lambda+1=0 \Leftrightarrow \lambda=1(\text { mit Vielfachheit } r=2) .
$$

Nach Folgerung 4.3.5 ist

$$
\widetilde{x}(n)=c_{1}+c_{2} \cdot n \quad \text { mit beliebigen } \quad c_{1}, c_{2} \in \mathbb{R}
$$

die allgemeine Lösung der entsprechenden homogenen Gleichung auf $\mathbb{N}_{0}$.

2) Wir haben $P_{0}(n)=2$. Da $\lambda=1$ eine Lösung mit Vielfachheit $r=2$ unserer charakteristischen Gleichung ist, suchen wir nach Bemerkung 4.5.2 eine partikuläre Lösung der inhomogenen Gleichung in der Form

$$
x^{*}(n)=n^{2} \cdot A=A n^{2} \quad \text { mit } A \in \mathbb{R} .
$$

Einsetzen in die inhomogene Gleichung (4.5.5) ergibt:

$$
A \cdot(n+2)^{2}-2 \cdot A \cdot(n+1)^{2}+A \cdot n^{2}=2 \quad \Leftrightarrow \quad 2 A=2 \quad \Leftrightarrow \quad A=1 .
$$

Dann ist

$$
x^{*}(n)=n^{2}
$$

eine partikuläre Lösung und

$$
x(n)=\widetilde{x}(n)+x^{*}(n)=c_{1}+c_{2} \cdot n+n^{2}
$$

die allgemeine Lösung der inhomogenen Gleichung (4.5.5) auf $\mathbb{N}_{0}$. 


\section{Beispiel 4.5.6}

$$
x(n+2)-3 x(n+1)-4 x(n)=(6 n-8) \cdot 2^{n}, \quad n \in \mathbb{N}_{0} .
$$

1) $x(n+2)-3 x(n+1)-4 x(n)=0$ ist die entsprechende homogene Gleichung. Die charakteristische Gleichung lautet

$$
\lambda^{2}-3 \lambda-4=0 \Leftrightarrow \lambda=4 \text { oder } \lambda=-1 \text { (jeweils mit Vielfachheit } r=1 \text { ) }
$$

Seien $\lambda_{1}:=4$ und $\lambda_{2}:=-1$. Nach Folgerung 4.3.3 ist

$$
\widetilde{x}(n)=c_{1} \cdot 4^{n}+c_{2} \cdot(-1)^{n} \quad \text { mit beliebigen } \quad c_{1}, c_{2} \in \mathbb{R}
$$

die allgemeine Lösung der entsprechenden homogenen Gleichung auf $\mathbb{N}_{0}$.

2) Wir haben $\gamma=2$ und $P_{1}(n)=6 n-8$. Da 2 keine Lösung unserer charakteristischen Gleichung ist, suchen wir nach Bemerkung 4.5.2 eine partikuläre Lösung der inhomogenen Gleichung in der Form

$$
x^{*}(n)=2^{n} \cdot(A n+B) \quad \text { mit } A, B \in \mathbb{R} .
$$

Einsetzen in die inhomogene Gleichung (4.5.6) ergibt:

$$
\begin{aligned}
& 2^{n+2} \cdot(A(n+2)+B) \\
& -3 \cdot 2^{n+1} \cdot(A(n+1)+B)-4 \cdot 2^{n} \cdot(A n+B)=(6 \cdot n-8) \cdot 2^{n} \\
& \underset{2^{n} \neq 0}{\Longleftrightarrow} \quad 4 \cdot(A(n+2)+B) \\
& -3 \cdot 2 \cdot(A(n+1)+B)-4 \cdot(A n+B)=6 n-8 \\
& (-6 A \cdot n)+(2 A-6 B)=6 n-8 .
\end{aligned}
$$

Ein Koeffizientenvergleich liefert:

$$
\left\{\begin{array} { l } 
{ - 6 A = 6 } \\
{ 2 A - 6 B = - 8 }
\end{array} \Longleftrightarrow \left\{\begin{array}{l}
A=-1 \\
B=1
\end{array}\right.\right.
$$

Dann ist

$$
x^{*}(n)=(-n+1) \cdot 2^{n}
$$


eine partikuläre Lösung und

$$
x(n)=\widetilde{x}(n)+x^{*}(n)=c_{1} \cdot 4^{n}+c_{2} \cdot(-1)^{n}+(-n+1) \cdot 2^{n}
$$

die allgemeine Lösung der inhomogenen Gleichung (4.5.6) auf $\mathbb{N}_{0}$.

\section{Beispiel 4.5.7}

$$
x(n+2)+5 x(n+1)+6 x(n)=(8 n-2) \cdot(-2)^{n}, \quad n \in \mathbb{N}_{0} .
$$

1) $x(n+2)+5 x(n+1)+6 x(n)=0$ ist die entsprechende homogene Gleichung. Die charakteristische Gleichung lautet $\lambda^{2}+5 \lambda+6=0 \Leftrightarrow \lambda=-2$ oder $\lambda=-3$ (jeweils mit Vielfachheit $r=1$ ).

Seien $\lambda_{1}:=-2$ und $\lambda_{2}:=-3$. Nach Folgerung 4.3.3 ist

$$
\widetilde{x}(n)=c_{1} \cdot(-2)^{n}+c_{2} \cdot(-3)^{n} \quad \text { mit beliebigen } \quad c_{1}, c_{2} \in \mathbb{R}
$$

die allgemeine Lösung der entsprechenden homogenen Gleichung auf $\mathbb{N}_{0}$.

2) Wir haben $\gamma=-2$ und $P_{1}(n)=8 n-2$. Da $\lambda=-2$ eine Lösung mit Vielfachheit $r=1$ unserer charakteristischen Gleichung ist, suchen wir nach Bemerkung 4.5.2 eine partikuläre Lösung der inhomogenen Gleichung in der Form

$$
x^{*}(n)=n^{1} \cdot(-2)^{n} \cdot(A n+B)=(-2)^{n} \cdot\left(A n^{2}+B n\right) \quad \text { mit } A, B \in \mathbb{R} .
$$

Einsetzen in die inhomogene Gleichung (4.5.7) ergibt:

$$
\begin{aligned}
& (-2)^{n+2} \cdot\left(A(n+2)^{2}+B(n+2)\right) \\
& +5 \cdot(-2)^{n+1} \cdot\left(A(n+1)^{2}+B(n+1)\right) \\
& +6 \cdot(-2)^{n} \cdot\left(A n^{2}+B n\right)=(8 n-2) \cdot(-2)^{n} \\
& \Longleftrightarrow_{(-2)^{n} \neq 0} 4 \cdot\left(A(n+2)^{2}+B(n+2)\right) \\
& -5 \cdot 2 \cdot\left(A(n+1)^{2}+B(n+1)\right) \\
& +6 \cdot\left(A n^{2}+B n\right)=8 n-2 \\
& (-4 A) \cdot n+(6 A-2 B)=8 n-2 .
\end{aligned}
$$


Ein Koeffizientenvergleich liefert:

$$
\left\{\begin{array} { l } 
{ - 4 A = 8 } \\
{ 6 A - 2 B = - 2 }
\end{array} \Longleftrightarrow \left\{\begin{array}{l}
A=-2 \\
B=-5
\end{array}\right.\right.
$$

Dann ist

$$
x^{*}(n)=\left(-2 n^{2}-5 n\right) \cdot(-2)^{n}
$$

eine partikuläre Lösung und

$$
\begin{aligned}
x(n) & =\widetilde{x}(n)+x^{*}(n) \\
& =c_{1} \cdot(-2)^{n}+c_{2} \cdot(-3)^{n}+\left(-2 n^{2}-5 n\right) \cdot(-2)^{n}
\end{aligned}
$$

die allgemeine Lösung der inhomogenen Gleichung (4.5.7) auf $\mathbb{N}_{0}$.

\section{Beispiel 4.5.8}

$$
x(n+2)+4 x(n)=17 n \cdot \sin \left(\frac{\pi}{4} \cdot n\right), \quad n \in \mathbb{N}_{0} .
$$

1) $x(n+2)+4 x(n)=0$ ist die entsprechende homogene Gleichung. Die charakteristische Gleichung lautet

$$
\lambda^{2}+4=0 \Leftrightarrow \lambda= \pm \mathrm{i} \cdot 2(\text { jeweils mit Vielfachheit } r=1) .
$$

Hier gilt $\alpha=0, \beta=2, \varphi=\arccos (0)=\frac{\pi}{2}, q=4$ und nach Folgerung 4.3.7 ist

$$
\begin{aligned}
\widetilde{x}(n) & =(4)^{\frac{n}{2}} \cdot\left(c_{1} \cdot \cos \left(\frac{\pi}{2} \cdot n\right)+c_{2} \cdot \sin \left(\frac{\pi}{2} \cdot n\right)\right) \\
& =2^{n} \cdot\left(c_{1} \cdot \cos \left(\frac{\pi}{2} \cdot n\right)+c_{2} \cdot \sin \left(\frac{\pi}{2} \cdot n\right)\right)
\end{aligned}
$$

mit beliebigen $c_{1}, c_{2} \in \mathbb{R}$ die allgemeine Lösung der entsprechenden homogenen Gleichung auf $\mathbb{N}_{0}$.

2) Wir haben $\psi=\frac{\pi}{4}, P(n) \equiv 0$ (Nullpolynom) und $Q_{1}(n)=17 n$.

Da $\cos \frac{\pi}{4} \pm \mathrm{i} \cdot \sin \frac{\pi}{4}=\frac{\sqrt{2}}{2} \pm \mathrm{i} \cdot \frac{\sqrt{2}}{2}$ keine Lösungen unserer charakteristischen Gleichung sind, suchen wir nach Bemerkung 4.5.2 eine partikuläre Lösung der inhomogenen Gleichung in der Form

$$
x^{*}(n)=(A n+B) \cdot \cos \left(\frac{\pi}{4} \cdot n\right)+(C n+D) \cdot \sin \left(\frac{\pi}{4} \cdot n\right)
$$

mit $A, B, C, D \in \mathbb{R}$. 
Einsetzen in die inhomogene Gleichung (4.5.8) ergibt:

$$
\begin{aligned}
&(A(n+2)+B) \cdot \cos \left(\frac{\pi}{4} \cdot(n+2)\right) \\
&+(C(n+2)+D) \cdot \sin \left(\frac{\pi}{4} \cdot(n+2)\right)+\left((A n+B) \cdot \cos \left(\frac{\pi}{4} \cdot n\right)\right. \\
&+4\left.+(C n+D) \cdot \sin \left(\frac{\pi}{4} \cdot n\right)\right)=17 n \cdot \sin \left(\frac{\pi}{4} \cdot n\right) \\
& \Leftrightarrow \quad(A(n+2)+B) \cdot(\left.\sin \left(\frac{\pi}{4} \cdot n\right)\right) \\
&+(C(n+2)+D) \cdot \cos \left(\frac{\pi}{4} \cdot n\right) \\
&+4 \cdot\left((A n+B) \cdot \cos \left(\frac{\pi}{4} \cdot n\right)\right. \\
&\left.+(C n+D) \cdot \sin \left(\frac{\pi}{4} \cdot n\right)\right)=17 n \cdot \sin \left(\frac{\pi}{4} \cdot n\right) \\
& \Leftrightarrow \quad(4 A n+4 B+C(n+2)+D) \cdot \cos \left(\frac{\pi}{4} \cdot n\right) \\
&+(4 C n+4 D-A(n+2)-B) \cdot \sin \left(\frac{\pi}{4} \cdot n\right)=17 n \cdot \sin \left(\frac{\pi}{4} \cdot n\right) .
\end{aligned}
$$

Ein Koeffizientenvergleich liefert:

$$
\left\{\begin{array} { l } 
{ 4 A + C = 0 } \\
{ 4 B + 2 C + D = 0 } \\
{ - A + 4 C = 1 7 } \\
{ - 2 A - B + 4 D = 0 }
\end{array} \Leftrightarrow \left\{\begin{array}{l}
A=-1 \\
B=-\frac{30}{17} \\
C=4 \\
D=-\frac{16}{17}
\end{array}\right.\right.
$$

Dann ist

$$
x^{*}(n)=\left(-n-\frac{30}{17}\right) \cdot \cos \left(\frac{\pi}{4} \cdot n\right)+\left(4 n-\frac{16}{17}\right) \cdot \sin \left(\frac{\pi}{4} \cdot n\right)
$$

eine partikuläre Lösung und

$$
\begin{aligned}
x(n)= & \widetilde{x}(n)+x^{*}(n) \\
=2^{n} & \cdot\left(c_{1} \cdot \cos \left(\frac{\pi}{2} \cdot n\right)+c_{2} \cdot \sin \left(\frac{\pi}{2} \cdot n\right)\right) \\
& \quad\left(-n-\frac{30}{17}\right) \cdot \cos \left(\frac{\pi}{4} \cdot n\right)+\left(4 n-\frac{16}{17}\right) \cdot \sin \left(\frac{\pi}{4} \cdot n\right)
\end{aligned}
$$

die allgemeine Lösung der inhomogenen Gleichung (4.5.8) auf $\mathbb{N}_{0}$. 


\section{Beispiel 4.5.9}

$$
x(n+2)+x(n)=\cos \left(\frac{\pi}{2} \cdot n\right), \quad n \in \mathbb{N}_{0} .
$$

1) $x(n+2)+x(n)=0$ ist die entsprechende homogene Gleichung. Die charakteristische Gleichung lautet

$$
\lambda^{2}+1=0 \Leftrightarrow \lambda= \pm \mathrm{i} \text { (jeweils mit Vielfachheit } r=1 \text { ). }
$$

Hier gilt $\alpha=0, \beta=1, \varphi=\arccos (0)=\frac{\pi}{2}, q=1$ und nach Folgerung 4.3 .7 ist

$$
\widetilde{x}(n)=c_{1} \cdot \cos \left(\frac{\pi}{2} \cdot n\right)+c_{2} \cdot \sin \left(\frac{\pi}{2} \cdot n\right)
$$

mit beliebigen $c_{1}, c_{2} \in \mathbb{R}$ die allgemeine Lösung der entsprechenden homogenen Gleichung auf $\mathbb{N}_{0}$.

2) Wir haben $\psi=\frac{\pi}{2}, P_{0}(n)=1$ und $Q(n) \equiv 0$ (Nullpolynom).

Da $\lambda=\cos \frac{\pi}{2} \pm \mathrm{i} \cdot \sin \frac{\pi}{2}= \pm \mathrm{i} \quad$ Lösungen (jeweils mit Vielfachheit $r=1$ ) unserer charakteristischen Gleichung sind, suchen wir nach Bemerkung 4.5.2 eine partikuläre Lösung der inhomogenen Gleichung in der Form

$$
x^{*}(n)=n^{1} \cdot\left(A \cdot \cos \left(\frac{\pi}{2} \cdot n\right)+B \cdot \sin \left(\frac{\pi}{2} \cdot n\right)\right) \quad \text { mit } A, B \in \mathbb{R} .
$$

Einsetzen in die inhomogene Gleichung (4.5.9) ergibt:

$$
\begin{aligned}
(n+2) \cdot\left(A \cdot \cos \left(\frac{\pi}{2} \cdot(n+2)\right)+B \cdot \sin \left(\frac{\pi}{2} \cdot(n+2)\right)\right) \\
+n \cdot\left(A \cdot \cos \left(\frac{\pi}{2} \cdot n\right)+B \cdot \sin \left(\frac{\pi}{2} \cdot n\right)\right)=\cos \left(\frac{\pi}{2} \cdot n\right) \\
\Leftrightarrow \quad(n+2) \cdot\left(-A \cdot \cos \left(\frac{\pi}{2} \cdot n\right)-B \cdot \sin \left(\frac{\pi}{2} \cdot n\right)\right) \\
+n \cdot\left(A \cdot \cos \left(\frac{\pi}{2} \cdot n\right)+B \cdot \sin \left(\frac{\pi}{2} \cdot n\right)\right)=\cos \left(\frac{\pi}{2} \cdot n\right) \\
\Leftrightarrow \quad-2 A \cdot \cos \left(\frac{\pi}{2} \cdot n\right)-2 B \cdot \sin \left(\frac{\pi}{2} \cdot n\right)=\cos \left(\frac{\pi}{2} \cdot n\right) .
\end{aligned}
$$

Ein Koeffizientenvergleich liefert:

$$
\left\{\begin{array} { l } 
{ - 2 A = 1 } \\
{ - 2 B = 0 }
\end{array} \Leftrightarrow \left\{\begin{array}{l}
A=-\frac{1}{2} \\
B=0
\end{array}\right.\right.
$$


Dann ist

$$
x^{*}(n)=-\frac{n}{2} \cdot \cos \left(\frac{\pi}{2} \cdot n\right)
$$

eine partikuläre Lösung und

$$
\begin{aligned}
x(n) & =\widetilde{x}(n)+x^{*}(n) \\
& =c_{1} \cdot \cos \left(\frac{\pi}{2} \cdot n\right)+c_{2} \cdot \sin \left(\frac{\pi}{2} \cdot n\right)-\frac{n}{2} \cdot \cos \left(\frac{\pi}{2} \cdot n\right)
\end{aligned}
$$

die allgemeine Lösung der inhomogenen Gleichung (4.5.9) auf $\mathbb{N}_{0}$.

\section{Beispiel 4.5.10}

$$
x(n+2)+2 x(n+1)+4 x(n)=2^{n} \cdot \sin \left(\frac{\pi}{2} \cdot n\right), \quad n \in \mathbb{N}_{0} .
$$

1) $x(n+2)+2 x(n+1)+4 x(n)=0$ ist die entsprechende homogene Gleichung. Die charakteristische Gleichung lautet

$$
\left.\lambda^{2}+2 \lambda+4=0 \Leftrightarrow \lambda=-1 \pm \mathrm{i} \cdot \sqrt{3} \text { (jeweils mit Vielfachheit } r=1\right) .
$$

Hier gilt $\alpha=-1, \beta=\sqrt{3}, \varphi=\arccos \left(-\frac{1}{2}\right)=\frac{2 \pi}{3}, q=4$ und nach Folgerung 4.3.7 ist

$$
\begin{aligned}
\widetilde{x}(n) & =4^{\frac{n}{2}} \cdot\left(c_{1} \cdot \cos \left(\frac{2 \pi}{3} \cdot n\right)+c_{2} \cdot \sin \left(\frac{2 \pi}{3} \cdot n\right)\right) \\
& =2^{n} \cdot\left(c_{1} \cdot \cos \left(\frac{2 \pi}{3} \cdot n\right)+c_{2} \cdot \sin \left(\frac{2 \pi}{3} \cdot n\right)\right)
\end{aligned}
$$

mit beliebigen $c_{1}, c_{2} \in \mathbb{R}$ die allgemeine Lösung der entsprechenden homogenen Gleichung auf $\mathbb{N}_{0}$.

2) Wir haben $\rho=2, \psi=\frac{\pi}{2}, P(n) \equiv 0$ (Nullpolynom) und $Q_{0}(n)=1$.

Da $2 \cdot\left(\cos \frac{\pi}{2} \pm \mathrm{i} \cdot \sin \frac{\pi}{2}\right)= \pm \mathrm{i} \cdot 2 \quad$ keine Lösungen unserer charakteristischen Gleichung sind, suchen wir nach Bemerkung 4.5.2 eine partikuläre Lösung der inhomogenen Gleichung in der Form

$$
x^{*}(n)=2^{n} \cdot\left(A \cdot \cos \left(\frac{\pi}{2} \cdot n\right)+B \cdot \sin \left(\frac{\pi}{2} \cdot n\right)\right) \quad \text { mit } A, B \in \mathbb{R} .
$$


Einsetzen in die inhomogene Gleichung (4.5.10) ergibt:

$$
\begin{aligned}
& 2^{(n+2)} \cdot\left(A \cdot \cos \left(\frac{\pi}{2} \cdot(n+2)\right)+B \cdot \sin \left(\frac{\pi}{2} \cdot(n+2)\right)\right) \\
& +2 \cdot 2^{n+1} \cdot\left(A \cdot \cos \left(\frac{\pi}{2} \cdot(n+1)\right)+B \cdot \sin \left(\frac{\pi}{2} \cdot(n+1)\right)\right) \\
& +4 \cdot 2^{n} \cdot\left(A \cdot \cos \left(\frac{\pi}{2} \cdot n\right)+B \cdot \sin \left(\frac{\pi}{2} \cdot n\right)\right)=2^{n} \cdot \sin \left(\frac{\pi}{2} \cdot n\right) \\
& \underset{2^{n} \neq 0}{\Longleftrightarrow} \quad 2^{2} \cdot\left(-A \cdot \cos \left(\frac{\pi}{2} \cdot n\right)-B \cdot \sin \left(\frac{\pi}{2} \cdot n\right)\right) \\
& +2 \cdot 2 \cdot\left(-A \cdot \sin \left(\frac{\pi}{2} \cdot n\right)+B \cdot \cos \left(\frac{\pi}{2} \cdot n\right)\right) \\
& +4 \cdot\left(A \cdot \cos \left(\frac{\pi}{2} \cdot n\right)+B \cdot \sin \left(\frac{\pi}{2} \cdot n\right)\right)=\sin \left(\frac{\pi}{2} \cdot n\right) \\
& 4 B \cdot \cos \left(\frac{\pi}{2} \cdot n\right)-4 A \cdot \sin \left(\frac{\pi}{2} \cdot n\right)=\sin \left(\frac{\pi}{2} \cdot n\right) .
\end{aligned}
$$

Ein Koeffizientenvergleich liefert:

$$
\left\{\begin{array} { l } 
{ 4 B = 0 } \\
{ - 4 A = 1 }
\end{array} \Leftrightarrow \left\{\begin{array}{l}
A=-\frac{1}{4} \\
B=0
\end{array}\right.\right.
$$

Dann ist

$$
x^{*}(n)=-\frac{2^{n}}{4} \cdot \cos \left(\frac{\pi}{2} \cdot n\right)=-2^{n-2} \cdot \cos \left(\frac{\pi}{2} \cdot n\right)
$$

eine partikuläre Lösung und

$$
\begin{aligned}
x(n) & =\widetilde{x}(n)+x^{*}(n) \\
& =2^{n} \cdot\left(c_{1} \cdot \cos \left(\frac{2 \pi}{3} \cdot n\right)+c_{2} \cdot \sin \left(\frac{2 \pi}{3} \cdot n\right)\right)-2^{n-2} \cdot \cos \left(\frac{\pi}{2} \cdot n\right)
\end{aligned}
$$

die allgemeine Lösung der inhomogenen Gleichung (4.5.10) auf $\mathbb{N}_{0}$. 


\section{Beispiel 4.5.11}

$$
\begin{aligned}
x(n+2)+9 x(n) & =3^{n+2} \cdot \cos \left(\frac{\pi}{2} \cdot n\right), & & n \in \mathbb{N}_{0} \\
\Longleftrightarrow \quad x(n+2)+9 x(n) & =3^{n} \cdot 9 \cdot \cos \left(\frac{\pi}{2} \cdot n\right), & & n \in \mathbb{N}_{0} .
\end{aligned}
$$

1) $x(n+2)+9 x(n)=0$ ist die entsprechende homogene Gleichung. Die charakteristische Gleichung lautet

$$
\lambda^{2}+9=0 \Leftrightarrow \lambda= \pm \mathrm{i} \cdot 3 \text { (jeweils mit Vielfachheit } r=1 \text { ). }
$$

Hier gilt $\alpha=0, \beta=3, \varphi=\arccos (0)=\frac{\pi}{2}, q=9$ und nach Folgerung 4.3.7 ist

$$
\begin{aligned}
\widetilde{x}(n) & =9^{\frac{n}{2}} \cdot\left(c_{1} \cdot \cos \left(\frac{\pi}{2} \cdot n\right)+c_{2} \cdot \sin \left(\frac{\pi}{2} \cdot n\right)\right) \\
& =3^{n} \cdot\left(c_{1} \cdot \cos \left(\frac{\pi}{2} \cdot n\right)+c_{2} \cdot \sin \left(\frac{\pi}{2} \cdot n\right)\right)
\end{aligned}
$$

mit beliebigen $c_{1}, c_{2} \in \mathbb{R}$ die allgemeine Lösung der entsprechenden homogenen Gleichung auf $\mathbb{N}_{0}$.

2) Wir haben $\rho=3, \psi=\frac{\pi}{2}, P_{0}(n)=9$ und $Q(n) \equiv 0$ (Nullpolynom).

Da $\lambda=3 \cdot\left(\cos \frac{\pi}{2} \pm \mathrm{i} \cdot \sin \frac{\pi}{2}\right)= \pm \mathrm{i} \cdot 3 \quad$ Lösungen (jeweils mit Vielfachheit $r=1$ ) unserer charakteristischen Gleichung sind, suchen wir nach Bemerkung 4.5.2 eine partikuläre Lösung der inhomogenen Gleichung in der Form

$$
x^{*}(n)=n^{1} \cdot 3^{n} \cdot\left(A \cdot \cos \left(\frac{\pi}{2} \cdot n\right)+B \cdot \sin \left(\frac{\pi}{2} \cdot n\right)\right) \quad \text { mit } A, B \in \mathbb{R} .
$$

Einsetzen in die inhomogene Gleichung (4.5.11) ergibt:

$$
\begin{aligned}
&(n+2) \cdot 3^{(n+2)} \cdot\left(A \cdot \cos \left(\frac{\pi}{2} \cdot(n+2)\right)+B\right.\left.\cdot \sin \left(\frac{\pi}{2} \cdot(n+2)\right)\right) \\
&+9 \cdot n \cdot 3^{n} \cdot\left(A \cdot \cos \left(\frac{\pi}{2} \cdot n\right)+B \cdot \sin \left(\frac{\pi}{2} \cdot n\right)\right)=3^{2} \cdot 3^{n} \cdot \cos \left(\frac{\pi}{2} \cdot n\right) \\
& 3^{\natural+2} \neq 0 \quad(n+2) \cdot\left(-A \cdot \cos \left(\frac{\pi}{2} \cdot n\right)-B \cdot \sin \left(\frac{\pi}{2} \cdot n\right)\right) \\
& \quad+n \cdot\left(A \cdot \cos \left(\frac{\pi}{2} \cdot n\right)+B \cdot \sin \left(\frac{\pi}{2} \cdot n\right)\right)=\cos \left(\frac{\pi}{2} \cdot n\right) \\
& \Longleftrightarrow \quad-2 A \cdot \cos \left(\frac{\pi}{2} \cdot n\right)-2 B \cdot \sin \left(\frac{\pi}{2} \cdot n\right)=\cos \left(\frac{\pi}{2} \cdot n\right)
\end{aligned}
$$


Ein Koeffizientenvergleich liefert:

$$
\left\{\begin{array} { l } 
{ - 2 A = 1 } \\
{ - 2 B = 0 }
\end{array} \Leftrightarrow \left\{\begin{array}{l}
A=-\frac{1}{2} \\
B=0
\end{array}\right.\right.
$$

Dann ist

$$
x^{*}(n)=-\frac{n}{2} \cdot 3^{n} \cdot \cos \left(\frac{\pi}{2} \cdot n\right)
$$

eine partikuläre Lösung und

$$
\begin{aligned}
x(n) & =\widetilde{x}(n)+x^{*}(n) \\
& =3^{n} \cdot\left(c_{1} \cdot \cos \left(\frac{\pi}{2} \cdot n\right)+c_{2} \cdot \sin \left(\frac{\pi}{2} \cdot n\right)\right)-\frac{n}{2} \cdot 3^{n} \cdot \cos \left(\frac{\pi}{2} \cdot n\right)
\end{aligned}
$$

die allgemeine Lösung der inhomogenen Gleichung (4.5.11) auf $\mathbb{N}_{0}$.

\section{Beispiel 4.5.12}

$$
x(n+2)+\sqrt{3} \cdot x(n+1)+x(n)=(-1)^{n} \cdot \sin \left(\frac{\pi}{6} \cdot n\right), \quad n \in \mathbb{N}_{0} .
$$

1) $x(n+2)+\sqrt{3} \cdot x(n+1)+x(n)=0$ ist die entsprechende homogene Gleichung. Die charakteristische Gleichung lautet $\lambda^{2}+\sqrt{3} \cdot \lambda+1=0 \Leftrightarrow \lambda=-\frac{\sqrt{3}}{2} \pm \mathrm{i} \cdot \frac{1}{2}($ jeweils mit Vielfachheit $r=1)$. Hier gilt $\alpha=-\frac{\sqrt{3}}{2}, \beta=\frac{1}{2}, \varphi=\arccos \left(-\frac{\sqrt{3}}{2}\right)=\frac{5 \pi}{6}, q=1$ und nach Folgerung 4.3.7 ist

$$
\widetilde{x}(n)=c_{1} \cdot \cos \left(\frac{5 \pi}{6} \cdot n\right)+c_{2} \cdot \sin \left(\frac{5 \pi}{6} \cdot n\right)
$$

mit beliebigen $c_{1}, c_{2} \in \mathbb{R}$ die allgemeine Lösung der entsprechenden homogenen Gleichung auf $\mathbb{N}_{0}$.

2) Weiter gilt:

$$
(-1)^{n} \cdot \sin \left(\frac{\pi}{6} \cdot n\right)=\sin \left(\left(\frac{\pi}{6}+\pi\right) \cdot n\right)=\sin \left(\frac{7 \pi}{6} \cdot n\right)
$$


somit erhalten wir:

$$
\begin{aligned}
x(n+2)+\sqrt{3} \cdot x(n+1)+x(n) & =(-1)^{n} \cdot \sin \left(\frac{\pi}{6} \cdot n\right) \\
\Longleftrightarrow \quad x(n+2)+\sqrt{3} \cdot x(n+1)+x(n) & =\sin \left(\frac{7 \pi}{6} \cdot n\right) .
\end{aligned}
$$

Für $\theta:=\frac{7 \pi}{6}$ gilt:

$$
\cos (\theta) \pm \mathrm{i} \cdot \sin (\theta)=\cos \left(\frac{7 \pi}{6}\right) \pm \mathrm{i} \cdot \sin \left(\frac{7 \pi}{6}\right)=-\frac{\sqrt{3}}{2} \mp \mathrm{i} \cdot \frac{1}{2}
$$

sind Lösungen (jeweils mit Vielfachheit $r=1$ ) der charakteristischen Gleichung

$$
\lambda^{2}+\sqrt{3} \cdot \lambda+1=0
$$

Nach den Bemerkungen 4.5.2 und 4.5.3 suchen wir somit eine partikuläre Lösung der inhomogenen Gleichung in der Form

$$
x^{*}(n)=n^{1} \cdot\left(A \cdot \cos \left(\frac{7 \pi}{6} \cdot n\right)+B \cdot \sin \left(\frac{7 \pi}{6} \cdot n\right)\right) \quad \text { mit } A, B \in \mathbb{R} .
$$

Einsetzen in die inhomogene Gleichung (4.5.12i) ergibt:

$$
\begin{gathered}
(n+2) \cdot\left(A \cdot \cos \left(\frac{7 \pi}{6} \cdot(n+2)\right)\right. \\
\left.+B \cdot \sin \left(\frac{7 \pi}{6} \cdot(n+2)\right)\right) \\
+\sqrt{3} \cdot(n+1) \cdot\left(A \cdot \cos \left(\frac{7 \pi}{6} \cdot(n+1)\right)\right. \\
\left.+B \cdot \sin \left(\frac{7 \pi}{6} \cdot(n+1)\right)\right) \\
+n \cdot\left(A \cdot \cos \left(\frac{7 \pi}{6} \cdot n\right)\right. \\
\left.+B \cdot \sin \left(\frac{7 \pi}{6} \cdot n\right)\right)=\sin \left(\frac{7 \pi}{6} \cdot n\right)
\end{gathered}
$$




$$
\begin{aligned}
& \Longleftrightarrow \\
& (n+2) \cdot\left(A \cdot \cos \left(\frac{7 \pi}{6} \cdot n+\frac{7 \pi}{3}\right)\right. \\
& \left.+B \cdot \sin \left(\frac{7 \pi}{6} \cdot n+\frac{7 \pi}{3}\right)\right) \\
& +\sqrt{3} \cdot(n+1) \cdot\left(A \cdot \cos \left(\frac{7 \pi}{6} \cdot n+\frac{7 \pi}{6}\right)\right. \\
& \left.+B \cdot \sin \left(\frac{7 \pi}{6} \cdot n+\frac{7 \pi}{6}\right)\right) \\
& +n \cdot\left(A \cdot \cos \left(\frac{7 \pi}{6} \cdot n\right)\right. \\
& \left.+B \cdot \sin \left(\frac{7 \pi}{6} \cdot n\right)\right)=\sin \left(\frac{7 \pi}{6} \cdot n\right) \\
& \Longleftrightarrow \\
& (n+2) \cdot A \cdot\left(\cos \left(\frac{7 \pi}{6} \cdot n\right) \cdot \frac{1}{2}-\sin \left(\frac{7 \pi}{6} \cdot n\right) \cdot \frac{\sqrt{3}}{2}\right) \\
& +(n+2) \cdot B \cdot\left(\sin \left(\frac{7 \pi}{6} \cdot n\right) \cdot \frac{1}{2}+\cos \left(\frac{7 \pi}{6} \cdot n\right) \cdot \frac{\sqrt{3}}{2}\right) \\
& +\sqrt{3} \cdot(n+1) \cdot A\left(\cos \left(\frac{7 \pi}{6} \cdot n\right) \cdot\left(-\frac{\sqrt{3}}{2}\right)-\sin \left(\frac{7 \pi}{6} \cdot n\right) \cdot\left(-\frac{1}{2}\right)\right) \\
& +\sqrt{3} \cdot(n+1) \cdot B\left(\sin \left(\frac{7 \pi}{6} \cdot n\right) \cdot\left(-\frac{\sqrt{3}}{2}\right)+\cos \left(\frac{7 \pi}{6} \cdot n\right) \cdot\left(-\frac{1}{2}\right)\right) \\
& +n \cdot\left(A \cdot \cos \left(\frac{7 \pi}{6} \cdot n\right)+B \cdot \sin \left(\frac{7 \pi}{6} \cdot n\right)\right)=\sin \left(\frac{7 \pi}{6} \cdot n\right) \\
& \left(-\frac{1}{2} \cdot A+\frac{\sqrt{3}}{2} \cdot B\right) \cdot \cos \left(\frac{7 \pi}{6} \cdot n\right) \\
& +\left(-\frac{\sqrt{3}}{2} \cdot A-\frac{1}{2} \cdot B\right) \cdot \sin \left(\frac{7 \pi}{6} \cdot n\right)=\sin \left(\frac{7 \pi}{6} \cdot n\right)
\end{aligned}
$$


Ein Koeffizientenvergleich liefert:

$$
\left\{\begin{array} { l } 
{ - \frac { 1 } { 2 } A + \frac { \sqrt { 3 } } { 2 } B = 0 } \\
{ - \frac { \sqrt { 3 } } { 2 } A - \frac { 1 } { 2 } B = 1 }
\end{array} \Leftrightarrow \left\{\begin{array}{l}
A=-\frac{\sqrt{3}}{2} \\
B=-\frac{1}{2}
\end{array}\right.\right.
$$

Dann ist

$$
x^{*}(n)=n \cdot\left(-\frac{\sqrt{3}}{2} \cdot \cos \left(\frac{7 \pi}{6} \cdot n\right)-\frac{1}{2} \cdot \sin \left(\frac{7 \pi}{6} \cdot n\right)\right)
$$

eine partikuläre Lösung und

$$
\begin{aligned}
x(n)=\widetilde{x}(n) & +x^{*}(n) \\
=c_{1} \cdot & \cos \left(\frac{5 \pi}{6} \cdot n\right)+c_{2} \cdot \sin \left(\frac{5 \pi}{6} \cdot n\right) \\
& +n \cdot\left(-\frac{\sqrt{3}}{2} \cdot \cos \left(\frac{7 \pi}{6} \cdot n\right)-\frac{1}{2} \cdot \sin \left(\frac{7 \pi}{6} \cdot n\right)\right)
\end{aligned}
$$

die allgemeine Lösung der inhomogenen Gleichung (4.5.12) auf $\mathbb{N}_{0}$.

\section{Beispiel 4.5.13}

$$
x(n+2)-5 x(n+1)+6 x(n)=\cos \left(\frac{\pi}{3} \cdot n\right)+n \cdot 3^{n}, \quad n \in \mathbb{N}_{0} .
$$

1) $x(n+2)-5 x(n+1)+6 x(n)=0$ ist die entsprechende homogene Gleichung. Die charakteristische Gleichung lautet

$$
\lambda^{2}-5 \lambda+6=0 \Leftrightarrow \lambda=2 \text { oder } \lambda=3 \text { (jeweils mit Vielfachheit } r=1 \text { ). }
$$

Seien $\lambda_{1}:=2, \lambda_{2}:=3$. Nach Folgerung 4.3.3 ist

$$
\widetilde{x}(n)=c_{1} \cdot 2^{n}+c_{2} \cdot 3^{n} \quad \text { mit beliebigen } \quad c_{1}, c_{2} \in \mathbb{R}
$$

die allgemeine Lösung der entsprechenden homogenen Gleichung auf $\mathbb{N}_{0}$. 
2) Nach Bemerkung 4.5.2 suchen wir eine partikuläre Lösung der inhomogenen Gleichung

$$
x(n+2)-5 x(n+1)+6 x(n)=\cos \left(\frac{\pi}{3} \cdot n\right)
$$

in der Form

$$
x_{1}^{*}(n)=A \cdot \cos \left(\frac{\pi}{3} \cdot n\right)+B \cdot \sin \left(\frac{\pi}{3} \cdot n\right) \quad \text { mit } A, B \in \mathbb{R} .
$$

Einsetzen in die inhomogene Gleichung (4.5.13a) ergibt:

$$
\begin{gathered}
A \cdot \cos \left(\frac{\pi}{3} \cdot(n+2)\right)+B \cdot \sin \left(\frac{\pi}{3} \cdot(n+2)\right) \\
-5 \cdot\left(A \cdot\left(\cos \left(\frac{\pi}{3} \cdot(n+1)\right)+B \cdot \sin \left(\frac{\pi}{3} \cdot(n+1)\right)\right)\right) \\
+6 \cdot\left(A \cdot \cos \left(\frac{\pi}{3} \cdot n\right)+B \cdot \sin \left(\frac{\pi}{3} \cdot n\right)\right)=\cos \left(\frac{\pi}{3} \cdot n\right) \\
\Leftrightarrow \quad A \cdot\left(\cos \left(\frac{\pi}{3} \cdot n\right) \cdot\left(-\frac{1}{2}\right)-\sin \left(\frac{\pi}{3} \cdot n\right) \cdot \frac{\sqrt{3}}{2}\right) \\
+B \cdot\left(\sin \left(\frac{\pi}{3} \cdot n\right) \cdot\left(-\frac{1}{2}\right)+\cos \left(\frac{\pi}{3} \cdot n\right) \cdot \frac{\sqrt{3}}{2}\right) \\
-5 \cdot\left(A \cdot\left(\cos \left(\frac{\pi}{3} \cdot n\right) \cdot \frac{1}{2}-\sin \left(\frac{\pi}{3} \cdot n\right) \cdot \frac{\sqrt{3}}{2}\right)\right. \\
\left.+B \cdot\left(\sin \left(\frac{\pi}{3} \cdot n\right) \cdot \frac{1}{2}+\cos \left(\frac{\pi}{3} \cdot n\right) \cdot \frac{\sqrt{3}}{2}\right)\right) \\
+6 \cdot\left(A \cdot \cos \left(\frac{\pi}{3} \cdot n\right)+B \cdot \sin \left(\frac{\pi}{3} \cdot n\right)\right)=\cos \left(\frac{\pi}{3} \cdot n\right) \\
\Leftrightarrow \quad(3 A-2 B \cdot \sqrt{3}) \cdot \cos \left(\frac{\pi}{3} \cdot n\right) \\
+(2 A \cdot \sqrt{3}+3 B) \cdot \sin \left(\frac{\pi}{3} \cdot n\right)=\cos \left(\frac{\pi}{3} \cdot n\right) .
\end{gathered}
$$

Ein Koeffizientenvergleich liefert:

$$
\left\{\begin{array} { l } 
{ 3 A - 2 B \cdot \sqrt { 3 } = 1 } \\
{ 2 A \cdot \sqrt { 3 } + 3 B = 0 }
\end{array} \Leftrightarrow \left\{\begin{array}{l}
A=\frac{1}{7} \\
B=-\frac{2 \sqrt{3}}{21}
\end{array}\right.\right.
$$


Dann ist

$$
x_{1}^{*}(n)=\frac{1}{7} \cdot \cos \left(\frac{\pi}{3} \cdot n\right)-\frac{2 \sqrt{3}}{21} \cdot \sin \left(\frac{\pi}{3} \cdot n\right)
$$

eine partikuläre Lösung der inhomogenen Gleichung (4.5.13a).

3) Nach Bemerkung 4.5.2 suchen wir eine partikuläre Lösung der inhomogenen Gleichung

$$
x(n+2)-5 x(n+1)+6 x(n)=n \cdot 3^{n} .
$$

in der Form

$$
x_{2}^{*}(n)=n^{1} \cdot(A n+B) \cdot 3^{n}=\left(A n^{2}+B n\right) \cdot 3^{n}, \quad \text { mit } A, B \in \mathbb{R} .
$$

Einsetzen in die inhomogene Gleichung (4.5.13b) ergibt:

$$
\begin{aligned}
& \left(A(n+2)^{2}+B(n+2)\right) \cdot 3^{n+2} \\
& -5 \cdot\left(A(n+1)^{2}+B(n+1)\right) \cdot 3^{n+1}+6 \cdot\left(A n^{2}+B n\right) \cdot 3^{n}=n \cdot 3^{n} \\
& \underset{3^{n} \neq 0}{\Longleftrightarrow} 9 \cdot\left(A\left(n^{2}+4 n+4\right)+B(n+2)\right. \\
& -15 \cdot\left(A\left(n^{2}+2 n+1\right)+B(n+1)\right)+6 \cdot\left(A n^{2}+B n\right)=n \\
& \Longleftrightarrow \quad 6 A n+(21 A+3 B)=n .
\end{aligned}
$$

Ein Koeffizientenvergleich liefert:

$$
\left\{\begin{array} { l } 
{ 6 A = 1 } \\
{ 2 1 A + 3 B = 0 }
\end{array} \Leftrightarrow \left\{\begin{array}{l}
A=\frac{1}{6} \\
B=-\frac{7}{6}
\end{array}\right.\right.
$$

Dann ist

$$
x_{2}^{*}(n)=\left(\frac{n^{2}}{6}-\frac{7 n}{6}\right) \cdot 3^{n}
$$

eine partikuläre Lösung der inhomogenen Gleichung (4.5.13b). 
4) Nach dem Superpositionsprinzip (Satz 4.4.6) ist

$$
x^{*}(n)=x_{1}^{*}(n)+x_{2}^{*}(n)
$$

eine partikuläre Lösung der inhomogenen Gleichung (4.5.13) und

$$
\begin{aligned}
x(n)=\widetilde{x}(n) & +x^{*}(n) \\
=c_{1} \cdot 2^{n}+c_{2} \cdot 3^{n} & \\
& \quad+\frac{1}{7} \cdot \cos \left(\frac{\pi}{3} \cdot n\right)-\frac{2 \sqrt{3}}{21} \cdot \sin \left(\frac{\pi}{3} \cdot n\right)+\left(\frac{n^{2}}{6}-\frac{7 n}{6}\right) \cdot 3^{n}
\end{aligned}
$$

die allgemeine Lösung der Gleichung (4.5.13) auf $\mathbb{N}_{0}$. 



\section{Schlusswort}

Wir haben in diesem Lehrbuch lineare Differential- und lineare Differenzengleichungen erster und zweiter Ordnung betrachtet. Gleichungen $n$-ter Ordnung, mit $n=3,4,5, \ldots$, lassen sich in analoger Weise zu den Betrachtungen der Gleichungen zweiter Ordnung untersuchen. 



\section{Anhang}

\section{Das Griechische Alphabet}

$\begin{array}{cccccccc}\text { Alpha } & \text { Beta } & \text { Gamma } & \text { Delta } & \text { Epsilon } & \text { Zeta } & \text { Eta } & \text { Theta } \\ A & B & \Gamma & \Delta & E & Z & H & \Theta \\ \alpha & \beta & \gamma & \delta & \varepsilon & \zeta & \eta & \theta\end{array}$

$\begin{array}{cccccccc}\text { Iota } & \text { Kappa } & \text { Lambda } & \text { My } & \text { Ny } & \mathbf{X i} & \text { Omikron } & \mathbf{P i} \\ I & K & \Lambda & M & N & \Xi & O & \Pi \\ \iota & \kappa & \lambda & \mu & \nu & \xi & O & \pi\end{array}$

Rho Sigma Tau Ypsilon Phi Chi Psi Omega

$\begin{array}{cccccccc}P & \Sigma & T & Y & \Phi & X & \Psi & \Omega \\ \rho & \sigma & \tau & u & \varphi & \chi & \psi & \omega\end{array}$




\section{Trigonometrische Formeln}

\section{Additionstheoreme}

(1) $\sin (\alpha+\beta)=\sin \alpha \cdot \cos \beta+\cos \alpha \cdot \sin \beta$

(2) $\sin (\alpha-\beta)=\sin \alpha \cdot \cos \beta-\cos \alpha \cdot \sin \beta$

(3) $\cos (\alpha+\beta)=\cos \alpha \cdot \cos \beta-\sin \alpha \cdot \sin \beta$

(4) $\cos (\alpha-\beta)=\cos \alpha \cdot \cos \beta+\sin \alpha \cdot \sin \beta$

\section{Umformungen von Summen in Produkte}

(1) $\sin \alpha+\sin \beta=2 \cdot \sin \frac{\alpha+\beta}{2} \cdot \cos \frac{\alpha-\beta}{2}$

(2) $\sin \alpha-\sin \beta=2 \cdot \sin \frac{\alpha-\beta}{2} \cdot \cos \frac{\alpha+\beta}{2}$

(3) $\cos \alpha+\cos \beta=2 \cdot \cos \frac{\alpha+\beta}{2} \cdot \cos \frac{\alpha-\beta}{2}$

(4) $\cos \alpha-\cos \beta=-2 \cdot \sin \frac{\alpha+\beta}{2} \cdot \sin \frac{\alpha-\beta}{2}$

\section{Umformungen von Produkten in Summen}
(1) $\sin \alpha \cdot \sin \beta=\frac{1}{2} \cdot(\cos (\alpha-\beta)-\cos (\alpha+\beta))$
(2) $\cos \alpha \cdot \cos \beta=\frac{1}{2} \cdot(\cos (\alpha-\beta)+\cos (\alpha+\beta))$
(3) $\sin \alpha \cdot \cos \beta=\frac{1}{2} \cdot(\sin (\alpha-\beta)+\sin (\alpha+\beta))$

\section{Die einfachsten trigonometrischen Gleichungen}
(1) $\sin x=a \Longleftrightarrow x=(-1)^{k} \cdot \arcsin a+\pi k$ mit $k \in \mathbb{Z}$, wenn $|a| \leqslant 1$ gilt
(2) $\cos x=a \Longleftrightarrow x= \pm \arccos a+2 \pi l$ mit $l \in \mathbb{Z}$, wenn $|a| \leqslant 1$ gilt
(3) $\tan x=a \Longleftrightarrow x=\arctan a+\pi m$ mit $m \in \mathbb{Z}$
(4) $\cot x=a \Longleftrightarrow x=\operatorname{arccot} a+\pi n$ mit $n \in \mathbb{Z}$ 


\section{Literaturverzeichnis}

[1] H. Heuser: Gewöhnliche Differentialgleichungen. 6. Auflage, Vieweg+Teubner Verlag, 2009. 



\section{Stichwortverzeichnis}

CASORATI-Determinante, 129

MoIvre, Satz von MoIvre, 127

EulER'sche Formel, 68

FibonAcci-Zahlen, 144

WronsKi-Determinante, 71

Lineare (gewöhnliche) Differentialgleichung erster Ordnung, 1 Anfangswertproblem, 1

Eindeutigkeit der Lösung, 12

Lineare (gewöhnliche) Differentialgleichung zweiter Ordnung, 65

Anfangswertproblem, 66

Lineare Differenzengleichung erster Ordnung, 33

Anfangswertproblem, 33

Konstruktion der Lösung, 47

Eindeutigkeit der Lösung des Anfangswertproblems, 33

Lineare Differenzengleichung zweiter Ordnung, 123

Anfangswertproblem, 123

Lineare homogene Differentialgleichung erster Ordnung, 2

allgemeine Lösung, 3

mit konstantem Koeffizienten, 6

Lineare homogene Differentialgleichung zweiter Ordnung, 66

allgemeine Lösung, 73

Fundamentalsystem, 71

mit konstanten Koeffizienten, 79

charakteristische Gleichung, 79 
Lineare homogene Differenzengleichung erster Ordnung, 35

allgemeine Lösung, 37

mit konstantem Koeffizienten, 40

Lineare homogene Differenzengleichung zweiter Ordnung, 126

allgemeine Lösung , 131

Fundamentalsystem, 129

mit konstanten Koeffizienten, 137

charakteristische Gleichung, 138

Lineare inhomogene Differentialgleichung erster Ordnung, 6

allgemeine Lösung, 8

mit konstantem Koeffizienten, 14

Superpositionsprinzip, 13

Variation der Konstanten, 10

Lineare inhomogene Differentialgleichung zweiter Ordnung, 95

allgemeine Lösung, 97

mit konstanten Koeffizienten, 102

Superpositionsprinzip, 101

Variation der Konstanten, 98

Lineare inhomogene Differenzengleichung erster Ordnung, 41

allgemeine Lösung, 43

mit konstanten Koeffizienten, 50

Superpositionsprinzip, 49

Variation der Konstanten, 45

Lineare inhomogene Differenzengleichung zweiter Ordnung, 147

allgemeine Lösung, 149

mit konstanten Koeffizienten, 156

Superpositionsprinzip, 156

Variation der Konstanten, 150 

In einer stärker computerisierten Welt finden Differential- und Differenzengleichungen immer mehr Anwendung. Das vorliegende Lehrbuch ist insbesondere für Studierende der ingenieurwissenschaftlichen, der informatikorientierten und der ökonomischen Studiengänge geeignet. Ausgewählte Kapitel sind auch für Schülerinnen und Schüler aus der Oberstufe mit den Leistungskursen Mathematik/Physik/Informatik interessant.

Der präsentierte Stoff entspricht einer zweistündigen Vorlesung im Grundlagenbereich, wobei Basis-Kenntnisse aus der Analysis und der Linearen Algebra vorausgesetzt sind. Die Autoren zeigen Parallelen bei den Untersuchungen von linearen Differential- und linearen Differenzengleichungen auf, wobei die Vorgehensweisen anhand von vielen Beispielen ausführlich illustriert werden. Es werden lineare Differential- und lineare Differenzengleichungen erster und zweiter Ordnung betrachtet, sowie den Leserinnen und Leser alle Werkzeuge für die Betrachtungen von Gleichungen höherer Ordnung zur Verfügung gestellt.

\section{Logos Verlag Berlin}

